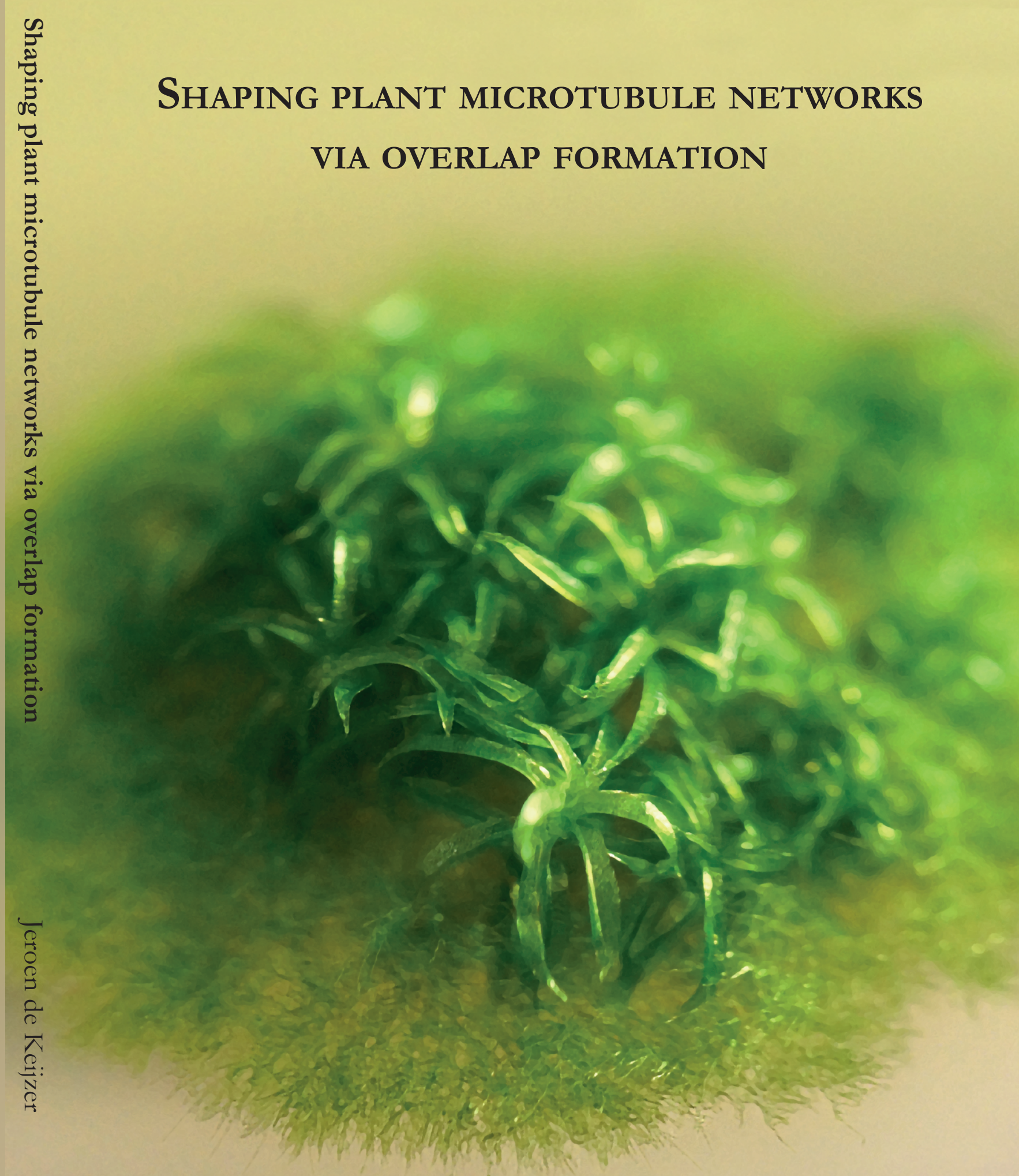

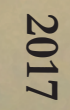




\section{Propositions}

1. A microtubule crosslinker/kinesin-4 module is a versatile and universal unit for structuring microtubule networks.

(this thesis)

2. Bipolar microtubule encounters give an opportunity to reinforce array unipolarity.

(this thesis)

3. Fluctuations in cytoskeletal organization enhance a cell's responsiveness to environmental cues.

4. Mutations causing mild, but experimentally perceivable phenotypical differences, are most useful in cell biological research.

5. The term 'lower plants' should refer to how often they are overlooked.

6. Videogames are the artform producing the contemporary masterpieces.

7. Nature management programmes are futile if foolishness of mankind is not simultaneously managed.

Propositions belonging to the thesis, entitled

"Shaping plant microtubule networks via overlap formation"

Jeroen de Keijzer

Wageningen, 17 November 2017. 


\section{Shaping PLANT MICROTUBULE NETWORKS VIA OVERLAP FORMATION}

Jeroen de Keijzer 


\section{Thesis committee}

\section{Promotor}

Prof. Dr M.E. Janson

Professor of Cell Biology

Wageningen University \& Research

\section{Co-promotor}

Dr M.J. Ketelaar

Assistant professor, Laboratory of Cell Biology

Wageningen University \& Research

\section{Other members}

Prof. Dr B.J.G. Scheres, Wageningen University \& Research

Dr T. Surrey, Francis Crick Institute, London, UK

Dr D. Bouchez, Institut Jean-Pierre Bourgin, Versailles, France

Prof. Dr E.J.G. Peterman, VU University Amsterdam

This research was conducted under the auspices of the Graduate School Experimental Plant Sciences 


\title{
SHAPING PLANT MicRotubULE NETWORKS VIA OVERLAP FORMATION
}

\author{
Jeroen de Keijzer
}

\section{Thesis}

submitted in fulfilment of the requirements for the degree of doctor

at Wageningen University

by the authority of the Rector Magnificus,

Prof. Dr A.P.J. Mol,

in the presence of the

Thesis Committee appointed by the Academic Board

to be defended in public

on Friday 17 November 2017

at 4 p.m. in the Aula. 
Jeroen de Keijzer

Shaping plant microtubule networks via overlap formation, 152 pages.

PhD thesis, Wageningen University, Wageningen, the Netherlands (2017)

With references, with summary in English

ISBN: 978-94-6343-810-0

DOI: $10.18174 / 424523$ 
Voor mijn ouders

A special feeling of gratitude to my parents 



\section{Table of contents}

$\begin{array}{lr}\text { Chapter } 1-\text { General introduction } & 9\end{array}$

Chapter 2 - Shortening of microtubule overlap regions defines $\quad 25$ membrane delivery sites during plant cytokinesis

Chapter 3 - Antiparallel microtubule overlaps position exocyst subunit Sec6 during plant cell division

Chapter 4 - Differential regulation of microtubule flux in the spindle and phragmoplast involves kinesin-4-mediated regulation of microtubule polymerization

Chapter 5 - Kinesin4-la restricts growth of antiparallel microtubule overlaps to enhance array polarity and prevent hyperparallelization for responsive tip growth guidance

Chapter 6 - General discussion: shaping microtubule networks for 123 plant cell division and expansion

Summary

Acknowledgements

List of publications

Curriculum vitae 



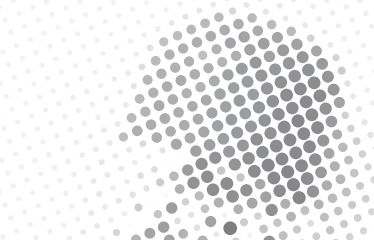

\section{Chapter 1 - General introduction}

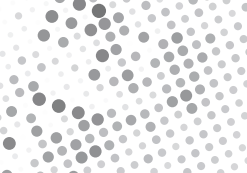

\section{Jeroen de Keijzer}

Laboratory of Cell Biology, Wageningen University, Droevendaalsesteeg 1, 6708 PB Wageningen, The Netherlands

[Parts of this chapter are based on (de Keijzeret al. (2014) Syst. Synth. Biol. 8, pp.187-194)]

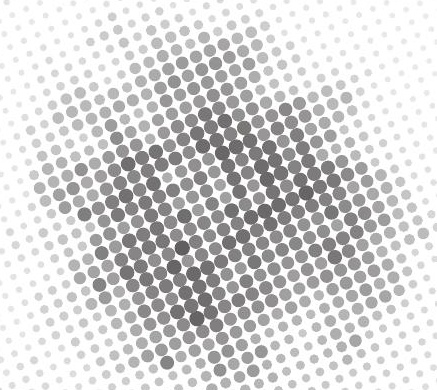

:898282889.

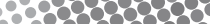

-9828

:8982828280.

:8288288898.

:-828

:828

:o828282888

-9828828889.

- 898288

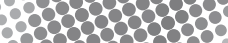

-8828282889.

.

$\because$ Beauty depends on size and order; hence an extremely minute creature could not be beautiful, for our vision becomes blurred as it approaches the point of imperceptibility, nor could an utterly huge creature be beautiful, for, unable to take it in all at once, the viewer finds that its unity and wholeness have escaped his field of vision"

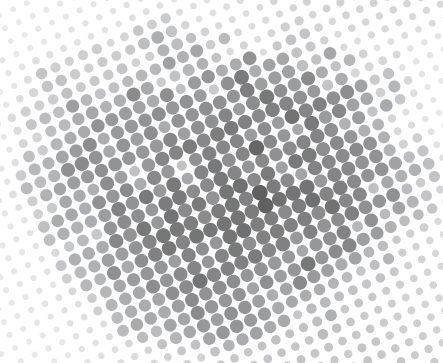

- Aristotle's Poetics, chapter 7, ca 335 bc, translated by J. Hutton

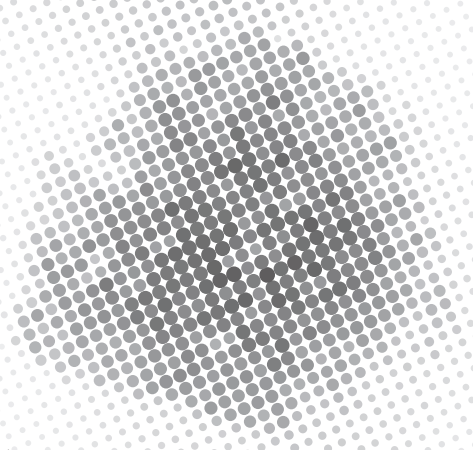




\section{Abstract}

Microtubules are dynamic polymers that are vital for a vast range of cellular functions. For virtually all of these functions microtubules are organized into specifically tailored networks. Plant cells present us with a remarkably diverse range of microtubule networks, one of which supports the unique manner in which these cells divide. The physical separation of two daughter cells at the end of cell division in plants is achieved by formation of an interior, disc-shaped membrane compartment called the cell plate that ultimately gives rise to a dividing cell wall segment. Cell plate construction starts in the centre of the cell and continues radially outwards until the cell plate fuses with the existing plasma membrane. Cell plate assembly is fuelled by fusion of vesicles that are targeted to the division plane by the microtubules of the phragmoplast apparatus. Phragmoplast microtubules are organized in a bipolar configuration that is propagated during radial expansion of the cell plate. In contrast to most animal cells, plants do not use centrosomes as foci of microtubule growth initiation. Instead, plant microtubule networks are striking examples of self-organizing systems that emerge from physically constrained interactions of dispersed microtubules. How microtubule-based activities including growth, shrinkage, severing, sliding, nucleation and bundling interrelate to jointly generate a required ordered structure is a fascinating but underexplored question. Evidence mounts that adapter proteins sense the local geometry of microtubules to locally modulate the activity of proteins involved in microtubule growth regulation and severing. One particular geometry that is a prime candidate for serving as an integrative hub in a bipolar configuration such as the phragmoplast, is the antiparallel overlap formed between opposing microtubules. The finding that proteins fostering this interaction are also important in interphase plant cells hints that overlaps may have roles in constructing microtubule networks beyond bipolar arrays. Insight into control of overlap formation gained from plants has broader relevance since many other microtubule assemblies encountered in eukaryotes rely on these structures as well.

\subsection{The principal microtubule networks during the plant cell cycle}

Since plant cells are virtually immobile within tissues owing to their encasement in a cell wall, control over division plane construction is an exceptionally important ingredient for tissue architecture in plants (Smolarkiewicz \& Dhonukshe 2013). Various microtubule networks constructed before and during cell division come into play for achieving this control. These networks are excellent examples of the remarkable flexibility with which plants cells can organize functional microtubule arrays in absence of major organizing centres. The principles that drive this organization are likely encountered in networks of other eukaryotic cells as well, bestowing broader relevance to insights gained from plants. In this section I will describe the successive networks generally executing plant cell division and the basic microtubule properties relevant for network construction. Then I will focus on the network involved in the final step of cell division, the cytokinetic phragmoplast, and the microtubule activities used to build and maintain this network. Finally, I will discuss the use of basal land plants in general and the moss Physcomitrella patens in particular as model systems for future mechanistic elucidation of activities driving microtubule network construction.

In the course of the cell cycle of land plants, four successive microtubule networks are generally encountered that control plant cell growth and division (Wasteneys 2002) (Figure 1). The location and orientation of the future division plane is established already before mitosis 
proper starts through the formation of a dense ring-like structure of microtubules, the preprophase band (PPB). The orientation of microtubules in this ring is adopted from the preceding aligned network of microtubules associated to the plasma membrane (Cleary et al. 1992; Vos et al. 2004). The still intact nucleus is located at the ring's centre and dictates the site of mitotic spindle construction (Masoud et al. 2013; Vos et al. 2004; Yamada \& Goshima 2017). As the PPB is disassembled, formation of a bipolar spindle starts for eventual segregation of the genetic material. Finally, the bipolar configuration of the spindle is reused in the phragmoplast (Lee \& Liu 2013). The phragmoplast deposits vesicles containing wall material to construct a new membrane enclosed precursor of the cross-wall, termed the cell plate, in the cell centre. The phragmoplast gradually expands in the direction of the plasma membrane and becomes ringshaped after microtubules at completed sections in the middle of the cell plate disassemble. The locus of the connection of the cell plate to the plasma membrane coincides with the position of the erstwhile PPB, making the PPB therefore a 100\% marker for the division plane (reviewed in Smith 2001; van Damme et al. 2007; Müller et al. 2009; van Damme 2009). Communication between the cytoplasmic phragmoplast and cortical markers, which location coincides with that of the former PPB, allow for control over symmetric and asymmetric cell divisions (Gallagher \& Smith 1999; Rasmussen et al. 2013; Smolarkiewicz \& Dhonukshe 2013). Recent findings showing that cortical markers can accumulate in lieu of a PPB strongly suggest that the PPB is not a specifier of the division plane per se, but rather an element within the division plane determination process that supplies refinement and stabilization (Schaefer et al. 2017). How the four networks exchange relevant positional and orientational information is summarized in Figure 1.

A striking feature of all microtubule networks in land plants, including the mitotic spindle and phragmoplast, is that their organization occurs in absence of centrosome-like organelles (Murata et al. 2007). Most microtubules in animal cells first appear at centrosomes, typically associated with the nuclear envelope, and then grow outwards. In contrast, microtubule growth in plants is initiated exclusively at spatially dispersed sites. This initiation, or nucleation, is catalysed by specialized nucleation complexes, and is the first of a number of microtubule-associated activities that occur in almost all networks (Teixidó-Travesa et al. 2012). After nucleation, periods of microtubule growth and shrinkage succeed each other in a GTP-dependent process termed dynamic instability (Gardner et al. 2013). Switches between the two states are termed catastrophes and rescues respectively. Dynamic instability is most pronounced at the so-called plusend of microtubules, but also the minus-end can have different states, being primarily static or shrinking (Ehrhardt \& Shaw 2006). Throughout the eukaryotic kingdom, the dynamic state of microtubules is controlled by a host of conserved proteins (Subramanian \& Kapoor 2012; Bowne-Anderson et al. 2015). Moreover, lateral associations between microtubules are facilitated by bundling proteins and specialized motor proteins. The latter activity can generate a force to slide microtubules along each other (Kapitein et al. 2005; Janson et al. 2007; Peterman \& Scholey 2009). Finally, existing microtubules can become severed to form two new microtubules (RollMecak \& McNally 2010). To mechanistically understand the formation of ordered microtubule networks for plant cell division it will be key to investigate microtubule nucleation, dynamics, bundling, sliding, and severing in an integral manner. For this thesis, I will focus on the plant phragmoplast and will examine how feedback between different microtubule activities is organized. 
1

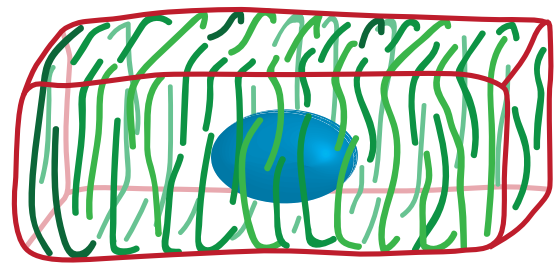

2

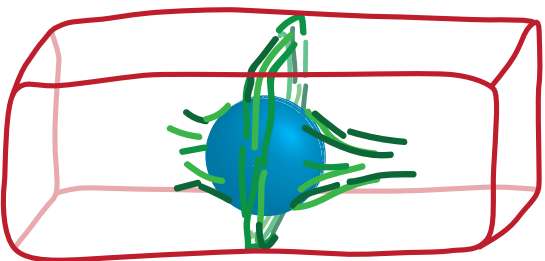

3

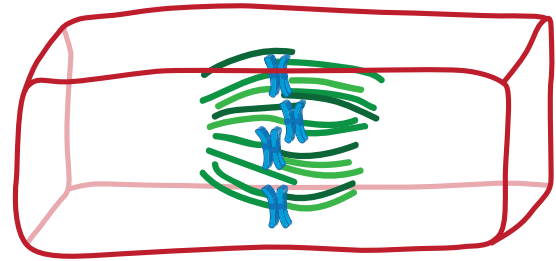

4

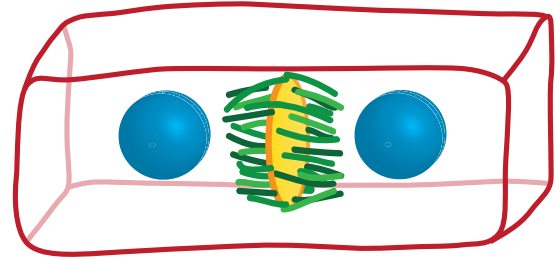

5

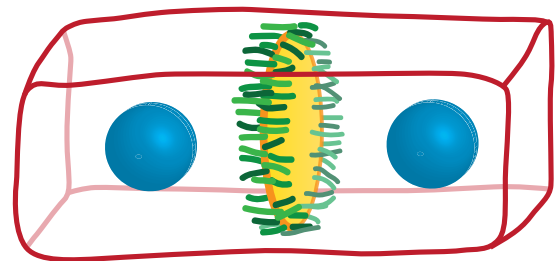

6

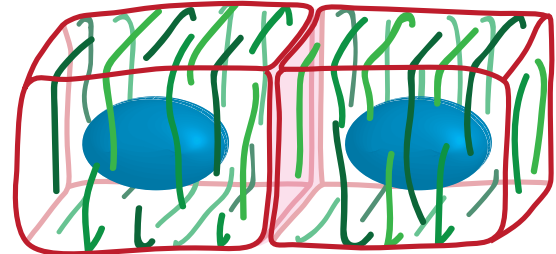

Figure 1 - Spatial relations between microtubule networks in plant cell division. The cortical microtubule array (1) develops into the dense ring-like preprophase band (2) whose location coincides with the cell nucleus (blue). Other microtubules that are initially formed around the nuclear envelope develop into a mitotic spindle that segregates sister chromatids (3). The phragmoplast forms out of the spindle remnant (4) and expands radially towards the location of the erstwhile pre-prophase band (5) leaving a finished cell plate in its wake (yellow) Once attached to the parental plasma membrane, the cell plate matures into a cell wall segment that separates the two newly formed daughter cells (6).

\subsection{Phragmoplast development and organisation}

The phragmoplast microtubule network comes forth from the bipolar mitotic spindle which in turn is initiated from a network of perinuclear microtubules. At the onset of mitosis a basket of microtubules appears around the still intact nuclear envelope and develops into a bipolar, noncentrosomal spindle after the nuclear envelope is broken down (Jensen \& Bajer 1973; De 
Mey et al. 1982; Vos et al. 2008; Nakaoka et al. 2012; Masoud et al. 2013; Yamada \& Goshima 2017). The mechanism of centrosome-independent spindle formation in plants is very relevant to other eukaryotic cells since their spindles more generally contain a subset of microtubules that are generated independently of centrosomes (Wainman et al. 2009; Uehara \& Goshima 2010). Spindles comprise two mirrored sets of polarized microtubules with plus-ends pointing towards the centre (Dhonukshe et al. 2006). Microtubule plus-ends either connect to their counterparts of the other spindle half or link chromatids to the spindle apparatus via the multiprotein kinetochore complex. Whereas the latter microtubules shorten during sister chromatid segregation (Jensen \& Bajer 1973), the former persist and form the template for the phragmoplast (Figure 2). As such, phragmoplast position, orientation and bipolarity are inherited from the spindle apparatus. These events are very much reminiscent of the animal cytokinetic microtubule array which also develops from remaining spindle microtubules (Glotzer 2009). During cellularization of certain multinucleated plant cells, spindles do not precede phragmoplast assembly. However, here another bipolar microtubule array is reported to form between nuclear surfaces to act as a precursor for the phragmoplast. Thus, in general, the phragmoplast maintains the bipolarity that is established by a preceding microtubule structure.
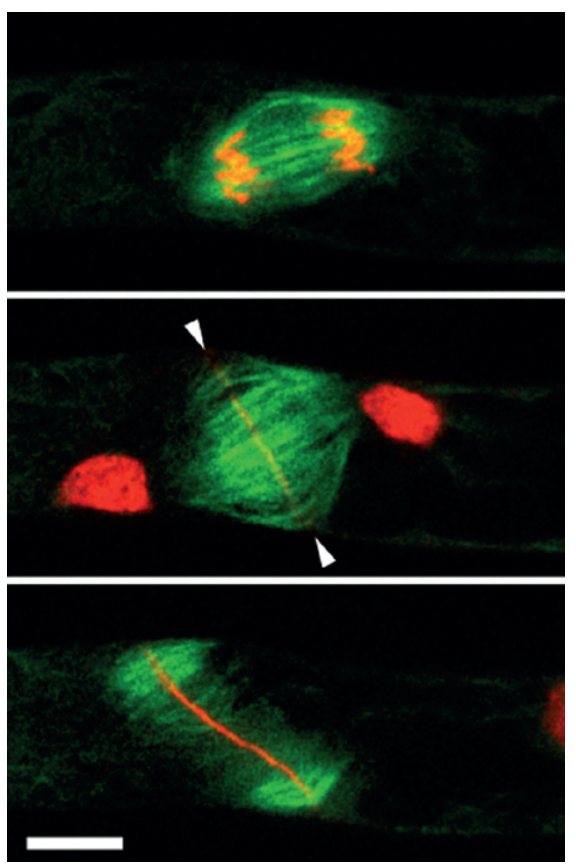

Figure 2 - Cytokinesis visualized by live cell imaging in the moss Physcomitrella patens. Microtubules are shown in green and chromatin and cell-plate material in red. Chromatids are segregated towards spindle poles and the remaining microtubules of the mitotic spindle (top panel) are used as template for the initial phragmoplast (middle panel; $6 \mathrm{~min}$ utes later). The reforming daughter nuclei are seen at the phragmoplast poles while accumulation of cell plate material is visible in the midplane of the phragmoplast where microtubules of opposite poles form antiparallel overlaps (arrowheads). Fifteen minutes later the central region of the cell plate has matured and the phragmoplast has expanded laterally (bottom panel). Microtubules were visualized using GFP fused to $\alpha$-tubulin, chromatin using mRFP fused to histone $2 B$ and the membranous material delivered to the cell plate with the lipophilic styryl dye FM4-64. The scalebar indicates $10 \mu \mathrm{m}$.

A new cell wall section is constructed at the equatorial plane (or midplane) of the phragmoplast (Figure 2, arrowheads), but how the factors involved in cell wall assembly are targeted to this site is largely unknown. Since microtubule plus-ends point towards the phragmoplast centre, an attractive targeting mechanism is directed transport of vesicular cargo along microtubules towards the division plane (Euteneuer \& Mcintosh 1980; Lee et al. 2001; Mcmichael \& Bednarek 2013). A subset of microtubules not only terminate near the phragmoplast centre, but extend across the division plane and meet with the opposing set to form short, antiparallel overlaps that coincide with the site of cell plate assembly. A key player for proper formation of these overlaps 
is the microtubule cross-linker MAP65, without which the two opposing sets of microtubules are not properly engaged, ultimately leading to cytokinesis failure (Müller et al. 2004; Ho, Hotta, Guo, et al. 2011; Kosetsu et al. 2013). A hypothesis that is further explored in this thesis is that besides aiding in maintenance of the overall bipolar structure of the phragmoplast, these cross-linked antiparallel overlaps may provide a very localized scaffold for directing cytokinetic processes, much akin to the stem body/Flemming body in the central spindle of animal cells (Otegui et al. 2005; Fuller et al. 2008; Glotzer 2009). Such a notion is supported by the delayed cell plate build-up when MAP65 is down-regulated even though a seemingly normal, bipolar phragmoplast is present (Kosetsu et al. 2013).

If there is indeed an intimate relationship between a microtubule overlap and the site of cell plate assembly, setting overlap length probably requires a certain degree of control. Recent work using various model systems provides insight into possible mechanisms for length regulation of overlaps in bipolar microtubule structures (Subramanian \& Kapoor 2012). A common concept encountered in these cases is that the dynamic parameters of microtubules engaged in an overlap are locally altered by an effector protein recruited to the overlap by microtubule cross-linkers of the Ase1/PRC1/MAP65 family. For instance, in yeast and human spindles the microtubule rescue factor CLASP is recruited (Bratman \& Chang 2007; Liu et al. 2009; Al-Bassam et al. 2010) and in case of the animal central spindle midzone a growth-inhibiting kinesin-4 is recruited by PRC1 (Bieling et al. 2010; Hu et al. 2011; Nunes Bastos et al. 2013). Large fluctuations in microtubule length are thus generally supressed in overlaps. Plus-end-directed kinesin motors at overlaps, some of which are possibly also recruited by cross-linkers, may furthermore contribute to length control by sliding microtubules apart (Nislow et al. 1992; Kapitein et al. 2005; Fu et al. 2009). In plants, microtubule growth in overlaps in combination with sliding by unknown motor proteins forms a plausible explanation for the continuous outward-directed flux of microtubule mass observed in both spindles and phragmoplasts (Asada et al. 1991; Dhonukshe et al. 2006; Murata et al. 2013). In the phragmoplast several kinesin motors have been identified that reside at the overlap zone and function in phragmoplast assembly and maintenance (Lee \& Liu 2000; Lee et al. 2007; Hiwatashi et al. 2008; Lee \& Liu 2013). Yet, how they contribute to the forces at play in the antiparallel overlaps of the phragmoplast is still not as well conceived as in other systems. It will be intriguing to learn how microtubule sliding, bundling and dynamics jointly control overlap length, especially since this may have direct bearing on the spatial regulation of cell plate assembly processes. The manner in which multiple overlaps are aligned to a continuous division plane is yet another intriguing and open question.

What drives the radial expansion of the phragmoplast towards the maternal cell wall? Phragmoplast microtubules themselves exhibit no or very little lateral movement (Yasuhara et al. 1993). Instead, existing microtubules are thought to disassemble at completed cell plate sections while newly nucleated microtubules form new overlaps at the leading edge, propagating the bipolar configuration laterally (Murata et al. 2013). In agreement, genetic or antibody interference of components of the microtubule-nucleation pathway severely inhibits phragmoplast expansion (Ho, Hotta, Guo, et al. 2011; Hotta et al. 2012; Nakaoka et al. 2012; Murata et al. 2013). Microtubule nucleation factors studied thus far localize throughout the phragmoplast with the exception of the equatorial region (Liu et al. 1993; Zeng et al. 2009; Ho et al. 2011; Nakaoka et al. 2012; Murata et al. 2013). Recent observations indicate that they may primarily reside on microtubule bundles that run perpendicular to the division plane (Murata et al. 2013). In the plant cortical array microtubule-dependent nucleation occurs at approximately $40^{\circ}$ with respect to the template microtubule (Murata et al. 2005; Murata \& Hasebe 2007; Chan 
et al. 2009). Though not yet directly shown, it is likely that microtubule branching angles in the phragmoplast also occur within a certain regime to reinforce its ruling directionality. Using meiotic frog extracts such a mechanism in which existing microtubules form a template for the ordered branching of new microtubules was elegantly demonstrated to operate in animal spindles (Petry et al. 2013). The ordered branching of microtubules may therefore be an important factor for maintaining network polarity during phragmoplast expansion.

Phragmoplast expansion at the periphery coincides with breakdown of microtubules in the phragmoplast centre, giving rise to a ring-shaped apparatus (Figure 1). How temporal regulation of microtubule breakdown is achieved is currently poorly understood, but likely depends on feedback from the state of cell plate assembly (Yasuhara \& Shibaoka 2000). The assembling of vesicles into a coherent cell plate is a gradual process that proceeds through phases distinguishable at the level of membrane morphology (Samuels et al. 1995), biochemical composition (Drakakaki 2015; Ahn et al. 2017) and vesicle dynamics (van Oostende-Triplet et al. 2017). Some of these characteristics may be used to perceive the degree of cell plate maturation and signal the dissolution of microtubules. A part of the signaling pathway that is involved in this task likely comprises a mitogen-activated protein kinase cascade that posttranslationally modifies MAP65 (Sasabe 2006; Sasabe \& Machida 2006; Sasabe \& Machida 2012).

Ultimately, the direction of expansion of the phragmoplast should be towards the sites on the parental cell membrane, occupied earlier by the PPB and demarcated by cortical markers (reviewed in Smith 2001; van Damme et al. 2007; van Damme 2009; Müller et al. 2009; Rasmussen et al. 2013). Early centrifugation experiments have demonstrated that dislocated phragmoplasts are able to move back towards the PPB-marked division zone, yet the nature of the driving forces remains elusive to date (Ota 1961; Mineyuki \& Gunning 1990). To induce changes in phragmoplast position, the marked cortical area could function as a signaling beacon, producing a gradient of a certain signaling molecule that affects phragmoplast dynamics. Alternatively, cytoskeletal filaments could form a physical link between the marked cortex and the phragmoplast (Lloyd \& Traas 1988; Sano et al. 2005; van Damme et al. 2007; Wu \& Bezanilla 2014). The selective presence or absence of various cytoskeletal-related proteins at the PPBmarked cortex is in support of such a linking mechanism. Proteins that are differentially located at the marked cortical zone include kinesin motors and actin itself (Cleary et al. 1992; Müller et al. 2006; Vanstraelen et al. 2006; van Damme et al. 2007; Miki et al. 2014). The identification of various other molecules that tune phragmoplast expansion will help to unravel the signaling network through which a flagged cortical domain can guide cell plate formation (Cleary \& Smith 1998; Müller et al. 2006; Walker et al. 2007; Xu et al. 2008).

\subsection{Microtubule activities for network construction: an outlook}

As exemplified by the phragmoplast, microtubules in eukaryotic cells exhibit very diverse and often flexible network geometries all involving a recurring set of activities: nucleation, dynamic instability, bundling, severing and sliding. Recent work on microtubule networks has provided insight into how feedback between these activities can be organized through adapter proteins (Figure 3A-C). Existing microtubules for example trigger nucleation of new microtubules through augmin-mediated recruitment of microtubule nucleation complexes leading to local microtubule amplification (Clausen \& Ribbeck 2007; Petry et al. 2013). Directionally biased microtubuledependent nucleation can reinforce an existing polarity in a spindle network (Kamasaki et al. 
2013). Another example of feedback involves the regulation of dynamic instability of two oppositely oriented microtubules engaged in microtubule overlap regions. Selective recruitment of CLASP or kinesin- 4 type proteins to these sites by bundling proteins can locally adapt the parameters of dynamic instability of the microtubules involved (Bratman \& Chang 2007; Bieling et al. 2010; Duellberg et al. 2013). Bundling proteins were also reported to bind motors, generating crosstalk between bundling and sliding action (Fu et al. 2009). Microtubule crossovers may furthermore trigger microtubule severing through SPIRAL2-mediated modulation of katanin activity (Figure3c) (Wightman et al. 2013). In generalized terms, adapter proteins may sense the local configuration of microtubules to provide feedback on microtubule network layout. In the aforementioned examples MAP65/Ase1/PRC1 family bundling proteins binding to antiparallel-oriented microtubules, and SPIRAL2 recruited to sites of microtubule cross-over fulfil the role of such adapter proteins (Janson et al. 2007; Subramanian et al. 2013; Wightman et al. 2013). The use of adapter proteins may enable a conserved set of proteins to be efficiently used in various network layouts and may facilitate fast switching between network architectures as observed in plant cell division. While antiparallel bundles in bipolar networks are obvious sites suitable for integration of multiple microtubule based activities, the finding that bundler proteins also support growth processes in interphase plant cells (Lucas et al. 2011), hints that overlaps may have roles in other network configurations as well.
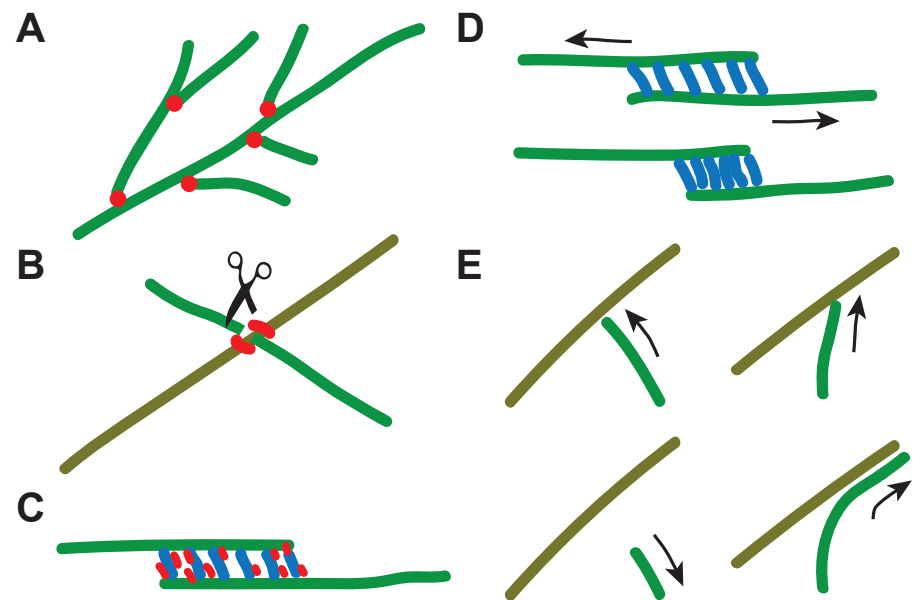

Figure 3 - Feedback between microtubule organization and microtubule activities. (A) Augmin-mediated branching of new microtubules from the sides of existing microtubules. (B) Points of microtubule crossover in plants are sites of microtubule severing by katanin, possibly regulated by SPIRAL2. (C) Crosslinking proteins in between overlapping microtubules recruit proteins that locally regulate dynamic instability or drive microtubule sliding. (D) Crosslinkers confined in between sliding microtubules are driven together by convening microtubule ends. (E) Existing microtubules obstruct the growth of incoming microtubules triggering shrinkage or deflect the growth axis leading to microtubule entrainment.

Besides microtubule configurations creating distinct biochemical environments, some forms of feedback could be more physical in origin. Well-established examples of this are microtubule overlap regions that are resistant to compressive forces generated by sliding through the packing of bundling proteins in the overlap and the induction of catastrophes or co-alignment through 
collisions among neighbouring microtubules (Figure 3D-E) (Allard et al. 2010; Braun et al. 2011). Cell geometry plays an additional role as shown by local cell wall curvature which may invoke microtubule catastrophes (Wasteneys \& Ambrose 2009). Therefore, work on diverse microtubule networks in animal, yeast, and plant cells, and insights gained from reconstituted in vitro systems highlight that the organization of networks can only be understood if multiple microtubule associated activities and physical concepts are integrated into our models.

\subsection{Physcomitrella patens as a model species for investigating plant microtubule network construction}

To further our understanding of organization and functioning of plant microtubule networks for cell division, development of new model systems will be instrumental. The PPB and phragmoplast microtubule arrays were innovations that probably arose in ancestral aquatic plants resembling currently living streptophyte algae, prior to traits enabling land colonization evolved (Figure 4) (Buschmann \& Zachgo 2016). Yet, much of our current notions on the components and mechanisms that build of these microtubule networks stem exclusively from flowering plants (angiosperms), the group that has brought forth most agronomically important crop plants. While studies on angiosperms have laid much of the groundwork in the field of plant cell division and are favourable for translating findings to a crop-context, involving more non-flowering plant models will confer two principal advantages. First, to get a grip on the evolutionary paths taken by the cellular and developmental machinery throughout the radiation of land plants (i.e. which of the observed mechanisms are ancestral and which are derived?), it is important to look into extant, early-divergent land plant taxa such as ferns and mosses (Figure 4) (Rensing 2017). Their study can expose the degree of conservation, adaptation and loss of traits, which ultimately also helps to define the predictive power of different plant model species. Secondly, non-flowering plants generally exhibit simpler body plans that offer attributes convenient for experimentation (e.g. space-efficient plant propagation or high accessibility by microscopy techniques). Despite the simpler morphology, in a growing number of cases the formation of similar plant structures in early-divergent plants and flowering plants turns out to be underpinned by conserved proteins. For example, the differentiation of water conducting cells as well as these forming the small pores regulating gas exchange between plant and atmosphere is controlled by conserved factors in mosses and angiosperms (Chater et al. 2011; Xu et al. 2014; Chater et al. 2016). The shared underlying genetic programmes highlight that diversification of model systems can broaden the palette of available techniques to understand common plant cellular phenomena.

Particularly for studying plant cell division the use of early-divergent land plants brings unique benefits. Cell division in flowering plants occurs in complex meristematic tissues in which microscopic observations of cell division are often hindered by several layers of overlaying cells. The simpler tissue architecture found in early-divergent plants enables the observation of cell division without interference of other cells. Moreover, these organisms have a prominent haploid phase in their life cycle, simplifying generation of loss-of-function mutants and fluorescent reporter gene fusions. Therefore, throughout this thesis I will use haploid, filamentous tissue of the moss Physcomitrella patens called caulonema.

For use as a model system in plant cell biology caulonema of $P$. patens garnered only limited attention after the moss was introduced from the Gransden Woods (UK) into the lab (Engel 
1968). However, during the past 20 years several merits of $P$. patens surfaced that raised the initially sparse regard. Transformation techniques were perfected (Nishiyama et al. 2000), gene-targeting through homologous recombination was discovered (Kammerer \& Cove 1996; Schaefer 2001) and molecular tools like RNAi became established (Bezanilla et al. 2003; Nakaoka et al. 2012). Furthermore, the predictable cell division pattern combined with a short cell cycle found in caulonema tissue of $P$. patens enabled large-scale studies into gene product localization and functioning during mitosis (Miki et al. 2014; Yamada et al. 2016). With these assets, P. patens proves to be an experimental platform with enormous potential for mechanistically dissecting the activities driving microtubule network construction for cell division.

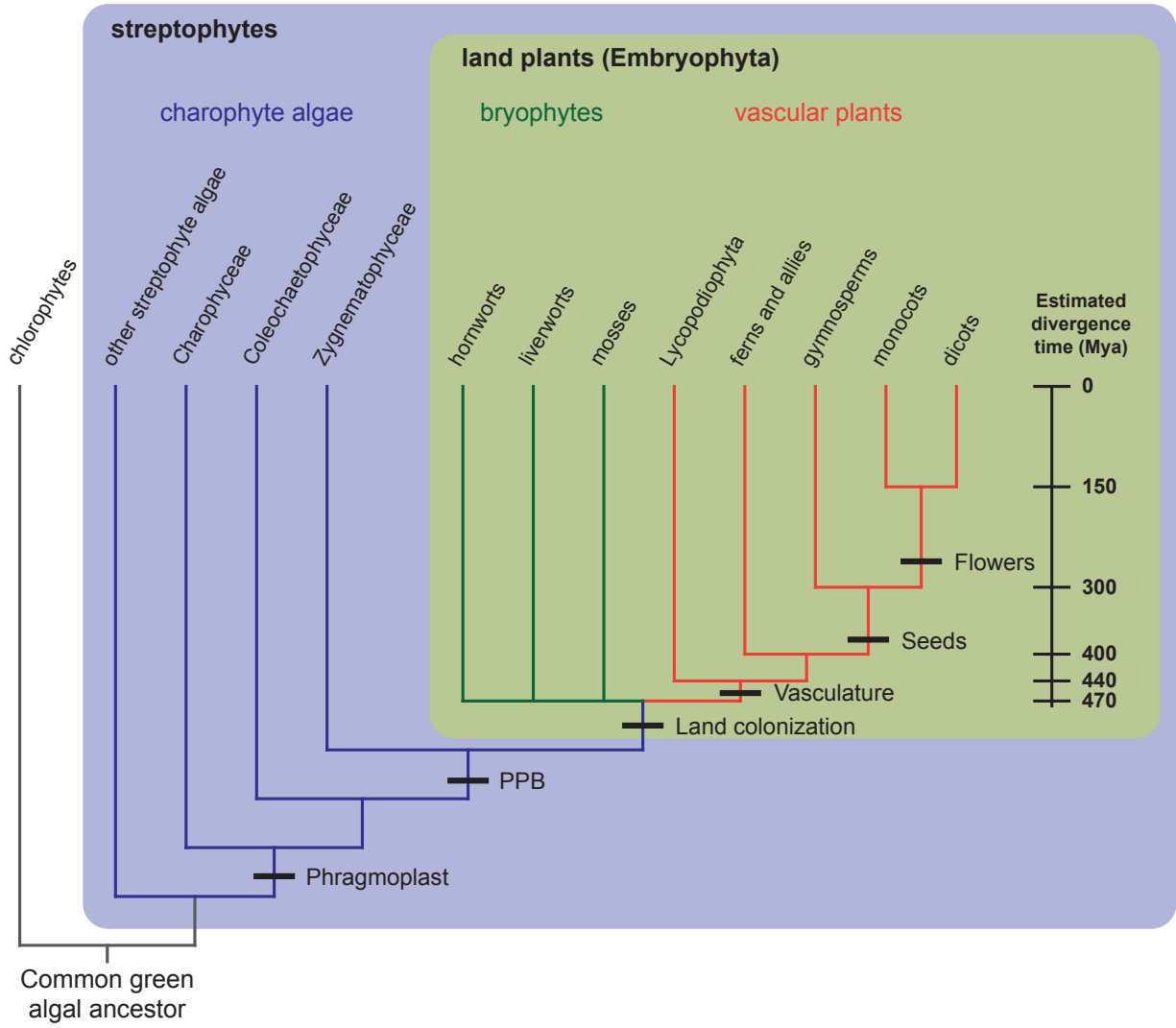

Figure 4 - Cladogram showing the phylogenetic relationship between major extant plant lineages. Black bars indicate key events during plant evolution, including the most likely appearance of the phragmoplast and PPB microtubule arrays (Buschmann \& Zachgo 2016). For land plants, branch lengths indicate approximate divergence times as shown on the right. The phylogenetic relationships are derived from a phylogenomics study by Wickett et al. (2014) and the divergence time estimates are from (Pires \& Dolan 2012).

Whilst this chapter focused on cell division, P. patens caulonemal cells similarly bring opportunities for illuminating mechanisms driving tip growth, the highly polarized expansion of a cell. Tip growth is found among various eukaryotes and is ubiquitously employed in the plant kingdom (Domozych et al. 2013; Rounds \& Bezanilla 2013). Also for this process a high degree of cytoskeletal organization is required, whereby a polarized microtubule network appears to 
play a role in defining the growth axis (Doonan et al. 1988; Ketelaar 2003; Hiwatashi et al. 2014). This array is different from other interphase arrays by also being composed of microtubules located in the endoplasm (Sieberer et al. 2005; Hiwatashi et al. 2014). What microtubule activities give rise to this 3-dimensional, polarized network remains currently obscure, although an early live-cell imaging study points towards microtubule bundle formation as a possible element (van Bruaene et al. 2004).

Thus, a single moss cell type presents us opportunities to look in detail at the construction mechanisms of diverse microtubule arrays that have bearing on essential cellular functions such as polar growth and division plane formation. This underscores that plants in general feature an intriguingly wide range of microtubule network layouts. These diverse networks may provide us unique openings to learn fundamental lessons about cytoskeletal self-organization in cell biology.

\subsection{Outline of this thesis}

This thesis will investigate how plant cells use microtubule networks to create unique subcellular environments for the specific purpose of guiding where building blocks for growth and division should be assembled. For this, we will focus on regions of antiparallel overlap formed between microtubules of opposing orientation, since these structures potentially serve as a nexus where multiple microtubule-based activities are integrated to shape functional microtubule arrays. Employing caulonemal cells of the moss Physcomitrella patens, we investigate the role of these overlaps in the phragmoplast microtubule array (Chapters 2-4) and in the interphase network supporting tip growth (Chapter 5).

Short regions of antiparallel overlap among microtubules of opposite poles exist in the phragmoplast of several plant species, including P. patens (Hiwatashi et al. 2008; Ho et al. 2011). While early electron-microscopy studies already noted that these overlaps are located in the same plane where membranous material for cell plate formation is localized (Hepler \& Jackson 1968), any causal ontogenetic relationship between these structures remained elusive. In chapter 2 we therefore hypothesize that the limited length of overlaps is essential for confined accumulation of cell plate membrane. To test this, inspired by observations on overlaps in animal cells, we first identify two kinesin- 4 motor proteins that shorten the domain of overlapping microtubules. We then show that upon over-elongation of phragmoplast overlaps, initial membrane accumulation occurs over a wider area, ultimately resulting in thick and misshapen walls dividing two daughter cells. This work thus demonstrates that overlaps provide a platform for the spatial orchestration of plant cytokinesis.

While these initial findings point to an intimate relationship between cell plate formation processes and microtubule overlap regions, how linkage is achieved at the molecular level remains obscure. In chapter 3 we therefore investigate the localization of one of the pieces of molecular machinery involved in the fusion of cell plate membrane, the exocyst vesicle tethering complex. We demonstrate that one of its subunits, but not several others, localizes to microtubule overlaps throughout cell division. We find that the presence and length of overlaps affect the targeting to this location. We also find indications that the subunit has other spatially and temporally regulated interactions during cytokinesis. We show that a conserved physical association with a membrane fusion component could be one of these interactions.

In chapter 4 we deepen the mechanistic understanding of the microtubule-based activities 
that shape phragmoplast microtubule overlaps and the division apparatus at large. For this, we focus on the cross-talk between growth activity at overlaps and the rate of pole-ward microtubule sliding, a concept rooted in observations on animal and yeast spindles. First, we characterize microtubule sliding in the $P$. patens division apparatus using photo-activation techniques and we demonstrate that kinesin- 4 in an in vivo context down-regulates microtubule growth in overlaps. Subsequently, we determine sliding speed in a kinesin- 4 knock-out situation and find that this is increased compared to wild-type. We further elucidate that lack of kinesin-4 growth regulation leads to longer pole-to-pole distances of the division apparatus. Together, these findings lead us to formulate a scenario in which the amount of poleward microtubule transport is co-determined by regulation of plus end growth at the phragmoplast centre and, together with tentative minus end breakdown processes at the poles, sets the length of the apparatus.

We noted that one of the kinesin-4 molecules studied in this thesis so far was not only present at phragmoplast microtubule overlaps, but also in the interphase microtubule network supporting tip growth. In chapter 5 we leverage this observation to expose that kinesin- 4 mediated length limitation of overlaps in the interphase network fosters an adaptable axis of tip growth that is responsive to environmental signals. To further understand how overlap length regulation connects to growth processes at the tip we perform a detailed analysis of plus-end growth in the interphase network. This reveals that kinesin- 4 action at overlaps modulates the microtubule array in two ways. On one hand it acts as a mechanism to reinforce existing array polarity, and on the other hand prevents hyperalignment of the microtubule network to the longitudinal cell axis. We propose that the latter activity is essential to allow efficient steering of a cell's growth axis.

In chapter 6 the findings on microtubule network organizing principles and their cellular role presented in this thesis are discussed. We relate our findings to other cell biological phenomena where microtubule networks provide cells with a sense of space. We broaden the notions we obtained on the role of dynamicity in the microtubule network in cells to other levels of biological integration.

\section{References}

Ahn, G. et al., 2017. SH3 domain-containing protein 2 plays a crucial role at the step of membrane tubulation during cell plate formation. The Plant Cell, 29(6), pp.1388-1405.

Al-Bassam, J. et al., 2010. CLASP promotes microtubule rescue by recruiting tubulin dimers to the microtubule. Developmental Cell, 19(2), pp.245-258.

Allard, J.F. et al., 2010. A mechanochemical model explains interactions between cortical microtubules in plants. Biophysical Journal, 99(4), pp.1082-1090.

Asada, T., Sonobe, S. \& Shibaoka, H., 1991. Microtubule translocation in the cytokinetic apparatus of cultured tobacco cells. Nature, 350, pp.238-241.

Bezanilla, M., Pan, A. \& Quatrano, R.S., 2003. RNA interference in the moss Physcomitrella patens. Plant Physiology, 133(2), pp.470-474.

Bieling, P., Telley, I. \& Surrey, T., 2010. A minimal midzone protein module controls formation and length of antiparallel microtubule overlaps. Cell, 142(3), pp.420-432.

Bowne-Anderson, H., Hibbel, A. \& Howard, J., 2015. Regulation of microtubule growth and catastrophe: Unifying theory and experiment. Trends in Cell Biology, 25(12), pp.769-779.

Bratman, S. V. \& Chang, F., 2007. Stabilization of overlapping microtubules by fission yeast CLASP. Developmental Cell, 13(6), pp.812-827.

Braun, M. et al., 2011. Adaptive braking by Ase1 prevents overlapping microtubules from sliding completely apart. Nature Cell Biology, 13(10), pp.1259-1264.

van Bruaene, N., Joss, G. \& van Oostveldt, P., 2004. Reorganization and in vivo dynamics of microtubules 
during Arabidopsis root hair development. Plant Physiology, 136(4), pp.3905-3919.

Buschmann, H. \& Zachgo, S., 2016. The evolution of cell division: From streptophyte algae to land plants. Trends in Plant Science, 21(10), pp.872-883.

Chan, J. et al., 2009. Arabidopsis cortical microtubules are initiated along, as well as branching from, existing microtubules. The Plant Cell, 12(8), pp.2298-2306.

Chater, C. et al., 2011. Regulatory mechanism controlling stomatal behavior conserved across 400 million years of land plant evolution. Current Biology, 21(12), pp.1025-1029.

Chater, C.C. et al., 2016. Origin and function of stomata in the moss Physcomitrella patens. Nature Plants, 2, e16179.

Clausen, T. \& Ribbeck, K., 2007. Self-organization of anastral spindles by synergy of dynamic instability, autocatalytic microtubule production, and a spatial signaling gradient. PloS one, 2(2), e244.

Cleary, A.L. et al., 1992. Microtubule and F-actin dynamics at the division site in living Tradescantia stamen hair cells. Journal of Cell Science, 103(4), pp.977-988.

Cleary, A.L. \& Smith, L.G., 1998. The Tangled1 gene is required for spatial control of cytoskeletal arrays associated with cell division during maize leaf development. The Plant Cell, 10(11), pp.1875-1888.

van Damme, D., 2009. Division plane determination during plant somatic cytokinesis. Current Opinion in Plant Biology, 12(6), pp.745-751.

van Damme, D., Vanstraelen, M. \& Geelen, D., 2007. Cortical division zone establishment in plant cells. Trends in Plant Science, 12(10), pp.458-464.

Dhonukshe, P., Vischer, N. \& Gadella, T.W.J., 2006. Contribution of microtubule growth polarity and flux to spindle assembly and functioning in plant cells. Journal of Cell Science, 119(15), pp.3193-3205.

Domozych, D., Fujimoto, C. \& LaRue, T., 2013. Polar expansion dynamics in the plant kingdom: A diverse and multifunctional journey on the path to pollen tubes. Plants, 2(1), pp.148-173.

Doonan, J., Cove, D.J. \& Lloyd, C., 1988. Microtubules and microfilaments in tip growth: evidence that microtubules impose polarity on protonemal growth in Physcomitrella patens. Journal of Cell Science, 89, pp.533-540.

Drakakaki, G., 2015. Polysaccharide deposition during cytokinesis: Challenges and future perspectives. Plant Science, 236, pp.177-184.

Duellberg, C. et al., 2013. End-binding proteins and Ase1/PRC1 define local functionality of structurally distinct parts of the microtubule cytoskeleton. Trends in Cell Biology, 23(2), pp.54-63.

Ehrhardt, D.W. \& Shaw, S.L., 2006. Microtubule dynamics and organization in the plant cortical array. Annual Review of Plant Biology, 57(1), pp.859-875.

Engel, P.P., 1968. The induction of biochemical and morphological mutants in the moss Physcomitrella patens. American Journal of Botany, 55(4), pp.438-446.

Euteneuer, U. \& Mcintosh, J.R., 1980. Polarity of midbody and phragmoplast microtubules. Journal of Cell Biology, 87, pp.509-515.

Fu, C. et al., 2009. Phospho-regulated interaction between kinesin-6 Klp9p and microtubule bundler Ase1p promotes spindle elongation. Developmental Cell, 17(2), pp.257-267.

Fuller, B.G. et al., 2008. Midzone activation of aurora B in anaphase produces an intracellular phosphorylation gradient. Nature, 453(7198), pp.1132-1136.

Gallagher, K. \& Smith, L.G., 1999. Discordia mutations specifically misorient asymmetric cell divisions during development of the maize leaf epidermis. Development, 126(20), pp.4623-4633.

Gardner, M.K., Zanic, M. \& Howard, J., 2013. Microtubule catastrophe and rescue. Current Opinion in Cell Biology, 25(1), pp.14-22.

Glotzer, M., 2009. The 3Ms of central spindle assembly: microtubules, motors and MAPs. Nature Reviews: Molecular Cell Biology, 10(1), pp.9-20.

Hepler, P.K. \& Jackson, W.T., 1968. Microtubules and early stages of cell-plate formation in the endosperm of Haemanthus katherinae Baker. Journal of Cell Biology, 38(2), pp.437-446.

Hiwatashi, Y. et al., 2008. Kinesins are indispensable for interdigitation of phragmoplast microtubules in the moss Physcomitrella patens. The Plant Cell, 20(11), pp.3094-3106.

Hiwatashi, Y., Sato, Y. \& Doonan, J.H., 2014. Kinesins have a dual function in organizing microtubules during both tip growth and cytokinesis in Physcomitrella patens. The Plant Cell, 26(3), pp.1256-1266.

Ho, C.-M.K., Hotta, T., Kong, Z., et al., 2011. Augmin plays a critical role in organizing the spindle and phragmoplast microtubule arrays in Arabidopsis. The Plant Cell, 23(7), pp.2606-2618.

Ho, C.-M.K., Hotta, T., Guo, F., et al., 2011. Interaction of antiparallel microtubules in the phragmoplast is mediated by the microtubule-associated protein MAP65-3 in Arabidopsis. The Plant Cell, 23(8), 
pp.2909-2923.

Hotta, T. et al., 2012. Characterization of the Arabidopsis augmin complex uncovers its critical function in the assembly of the acentrosomal spindle and phragmoplast microtubule arrays. The Plant Cell, 24(4), pp.1494-1509.

Hu, C.-K. et al., 2011. KIF4 regulates midzone length during cytokinesis. Current Biology, 21(10), pp.815824.

Janson, M.E. et al., 2007. Crosslinkers and motors organize dynamic microtubules to form stable bipolar arrays in fission yeast. Cell, 128(2), pp.357-368.

Jensen, C. \& Bajer, A., 1973. Spindle dynamics and arrangement of microtubules. Chromosoma, 44(1), pp.7389.

Kamasaki, T. et al., 2013. Augmin-dependent microtubule nucleation at microtubule walls in the spindle. Journal of Cell Biology, 202(1), pp.25-33.

Kammerer, W. \& Cove, D.J., 1996. Genetic analysis of the effects of re-transformation of transgenic lines of the moss Physcomitrella patens. Molecular \& General Genetics, 250(3), pp.380-382.

Kapitein, L.C. et al., 2005. The bipolar mitotic kinesin Eg5 moves on both microtubules that it crosslinks. Nature, 435(7038), pp.114-118.

de Keijzer, J., Mulder, B.M. \& Janson, M.E., 2014. Microtubule networks for plant cell division. Systems and Synthetic Biology, 8(3), pp.187-194.

Ketelaar, T., 2003. Unstable F-actin specifies the area and microtubules direction of cell expansion in Arabidopsis root hairs. The Plant Cell, 15(1), pp.285-292.

Kosetsu, K. et al., 2013. MICROTUBULE-ASSOCIATED PROTEIN65 is essential for maintenance of phragmoplast bipolarity and formation of the cell plate in Physcomitrella patens. The Plant Cell, 25(11), pp.4479-4492.

Lee, Y.-R.J., Li, Y. \& Liu, B., 2007. Two Arabidopsis phragmoplast-associated kinesins play a critical role in cytokinesis during male gametogenesis. The Plant Cell, 19(8), pp.2595-2605.

Lee, Y.-R.J. \& Liu, B., 2013. The rise and fall of the phragmoplast microtubule array. Current Opinion in Plant Biology, 16(6), pp.757-763.

Lee, Y.J., Giang, H.M. \& Liu, B., 2001. A novel plant kinesin-related protein specifically associates with the phragmoplast organelles. The Plant Cell, 13(11), pp.2427-2439.

Lee, Y.R. \& Liu, B., 2000. Identification of a phragmoplast-associated kinesin-related protein in higher plants. Current Biology, 10(13), pp.797-800.

Liu, B. et al., 1993. A gamma-tubulin-related protein associated with the microtubule arrays of higher plants in a cell cycle-dependent manner. Journal of Cell Science, 104(4), pp.1217-1228.

Liu, J. et al., 2009. PRC1 cooperates with CLASP1 to organize central spindle plasticity in mitosis. The Journal of Biological Chemistry, 284(34), pp.23059-23071.

Lloyd, C.W. \& Traas, J.A., 1988. The role of F-actin in determining the division plane of carrot suspension cells: Drug studies. Development, 221, pp.211-221.

Lucas, J.R. et al., 2011. Microtubule-associated proteins MAP65-1 and MAP65-2 positively regulate axial cell growth in etiolated Arabidopsis hypocotyls. The Plant Cell, 23(5), pp.1889-1903.

Masoud, K. et al., 2013. Microtubule nucleation and establishment of the mitotic spindle in vascular plant cells. The Plant Journal, 75(2), pp.245-257.

Mcmichael, C.M. \& Bednarek, S.Y., 2013. Cytoskeletal and membrane dynamics during higher plant cytokinesis. New Phytologist, 197(2013), pp.1039-1057.

De Mey, J. et al., 1982. Visualization of microtubules in interphase and mitotic plant cells of Haemanthus endosperm with the immuno-gold staining method. Proceedings of the National Academy of Sciences of the United States of America, 79(6), pp.1898-1902.

Miki, T. et al., 2014. Endogenous localizome identifies 43 mitotic kinesins in a plant cell. Proceedings of the National Academy of Sciences of the United States of America, 111(11), pp.1053-1061.

Mineyuki, Y. \& Gunning, B.E.S., 1990. A role for preprophase bands of microtubules in maturation of new cell walls, and a general proposal on the function of preprophase band sites in cell division in higher plants. Journal of Cell Science, 97(3), pp.527-537.

Müller, S. et al., 2004. The plant microtubule-associated protein AtMAP65-3/PLE is essential for cytokinetic phragmoplast function. Current Biology, 14(5), pp.412-417.

Müller, S., Han, S. \& Smith, L.G., 2006. Two kinesins are involved in the spatial control of cytokinesis in Arabidopsis thaliana. Current Biology, 16(9), pp.888-894.

Müller, S., Wright, A.J. \& Smith, L.G., 2009. Division plane control in plants: new players in the band. Trends 
in Cell Biology, 19(4), pp.180-188.

Murata, T. et al., 2007. How do plants organize microtubules without a centrosome? Journal of Integrative Plant Biology, 49(8), pp.1154-1163.

Murata, T. et al., 2013. Mechanism of microtubule array expansion in the cytokinetic phragmoplast. Nature Communications, 4, p.1967.

Murata, T. et al., 2005. Microtubule-dependent microtubule nucleation based on recruitment of gammatubulin in higher plants. Nature Cell Biology, 7(10), pp.961-968.

Murata, T. \& Hasebe, M., 2007. Microtubule-dependent microtubule nucleation in plant cells. Journal of Plant Research, 120(1), pp.73-78.

Nakaoka, Y. et al., 2012. An inducible RNA interference system in Physcomitrella patens reveals a dominant role of augmin in phragmoplast microtubule generation. The Plant Cell, 24(4), pp.1478-1493.

Nishiyama, T. et al., 2000. Tagged mutagenesis and gene-trap in the moss, Physcomitrella patens by shuttle mutagenesis. DNA Research, 7(1), pp.9-17.

Nislow, C. et al., 1992. A plus-end-directed motor enzyme that moves antiparallel microtubules in vitro localizes to the interzone of mitotic spindles. Nature, 359, pp.543-547.

Nunes Bastos, R. et al., 2013. Aurora B suppresses microtubule dynamics and limits central spindle size by locally activating KIF4A. Journal of Cell Biology, 202(4), pp.605-621.

van Oostende-Triplet, C. et al., 2017. Vesicle dynamics during plant cell cytokinesis reveals distinct developmental phases. Plant Physiology, 174(3), pp.1544-1558.

Ota, T., 1961. The role of cytoplasm in cytokinesis of plant cells. Cytologia, 26(3), pp.428-447.

Otegui, M.S., Verbrugghe, K.J. \& Skop, A.R., 2005. Midbodies and phragmoplasts: analogous structures involved in cytokinesis. Trends in Cell Biology, 15(8), pp.404-413.

Peterman, E.J.G. \& Scholey, J.M., 2009. Mitotic microtubule crosslinkers: Insights from mechanistic studies. Current Biology, 19(23), pp.1089-1094.

Petry, S. et al., 2013. Branching microtubule nucleation in Xenopus egg extracts mediated by augmin and TPX2. Cell, 152(4), pp.768-777.

Pires, N.D. \& Dolan, L., 2012. Morphological evolution in land plants: new designs with old genes. Philosophical Transactions of the Royal Society B: Biological Sciences, 367(1588), pp.508-518.

Rasmussen, C.G., Wright, A.J. \& Müller, S., 2013. The role of the cytoskeleton and associated proteins in determination of the plant cell division plane. The Plant Journal, 75(2), pp.258-269.

Rensing, S.A., 2017. Why we need more non-seed plant models. New Phytologist, 216(2), pp.355-360.

Roll-Mecak, A. \& McNally, FJ., 2010. Microtubule-severing enzymes. Current Opinion in Cell Biology, 22(1), pp.96-103.

Rounds, C.M. \& Bezanilla, M., 2013. Growth mechanisms in tip-growing plant cells. Annual Review of Plant Biology, 64(1), pp.243-265.

Samuels, A.L., Giddings, T.H. \& Staehelin, L.A., 1995. Cytokinesis in tobacco BY-2 and root tip cells: a new model of cell plate formation in higher plants. Journal of Cell Biology, 130(6), pp.1345-1357.

Sano, T. et al., 2005. Appearance of actin microfilament "twin peaks" in mitosis and their function in cell plate formation, as visualized in tobacco BY-2 cells expressing GFP-fimbrin. The Plant Journal, 44(4), pp.595-605.

Sasabe, M., 2006. Phosphorylation of NtMAP65-1 by a MAP kinase down-regulates its activity of microtubule bundling and stimulates progression of cytokinesis of tobacco cells. Genes \& Development, 20(8), pp.1004-1014.

Sasabe, M. \& Machida, Y., 2006. MAP65: a bridge linking a MAP kinase to microtubule turnover. Current Opinion in Plant Biology, 9(6), pp.563-570.

Sasabe, M. \& Machida, Y., 2012. Regulation of organization and function of microtubules by the mitogenactivated protein kinase cascade during plant cytokinesis. Cytoskeleton, 69(11), pp.913-918.

Schaefer, D.G., 2001. Gene targeting in Physcomitrella patens. Current Opinion in Plant Biology, 4(2), pp.143-150.

Schaefer, E. et al., 2017. The preprophase band of microtubules controls the robustness of division orientation in plants. Science, 356(6334), pp.186-189.

Sieberer, B.J. et al., 2005. Microtubules guide root hair tip growth. New Phytologist, 167(3), pp.711-719.

Smith, L.G., 2001. Plant cell division: building walls in the right places. Nature Reviews: Molecular Cell Biology, 2(1), pp.33-39.

Smolarkiewicz, M. \& Dhonukshe, P., 2013. Formative cell divisions: principal determinants of plant morphogenesis. Plant \& Cell Physiology, 54(3), pp.333-342.

Subramanian, R. et al., 2013. Marking and measuring single microtubules by PRC1 and kinesin-4. Cell, 
154(2), pp.377-390.

Subramanian, R. \& Kapoor, T.M., 2012. Building complexity: insights into self-organized assembly of microtubule-based architectures. Developmental Cell, 23(5), pp.874-885.

Teixidó-Travesa, N., Roig, J. \& Lüders, J., 2012. The where, when and how of microtubule nucleation - one ring to rule them all. Journal of Cell Science, 125(19), pp.4445-4456.

Uehara, R. \& Goshima, G., 2010. Functional central spindle assembly requires de novo microtubule generation in the interchromosomal region during anaphase. Journal of Cell Biology, 191(2), pp.259-267.

Vanstraelen, M. et al., 2006. Cell cycle-dependent targeting of a kinesin at the plasma membrane demarcates the division site in plant cells. Current Biology, 16(3), pp.308-314.

Vos, J.W. et al., 2008. The plant TPX2 protein regulates prospindle assembly before nuclear envelope breakdown. The Plant Cell, 20(10), pp.2783-2797.

Vos, J.W., Dogterom, M. \& Emons, A.M.C., 2004. Microtubules become more dynamic but not shorter during preprophase band formation: A possible "search-and-capture" mechanism for microtubule translocation. Cell Motility and the Cytoskeleton, 57(4), pp.246-258.

Wainman, A. et al., 2009. A new Augmin subunit, Msd1, demonstrates the importance of mitotic spindletemplated microtubule nucleation in the absence of functioning centrosomes. Genes \& Development, 23(16), pp.1876-1881.

Walker, K.L. et al., 2007. Arabidopsis TANGLED identifies the division plane throughout mitosis and cytokinesis. Current Biology, 17(21), pp.1827-1836.

Wasteneys, G.O., 2002. Microtubule organization in the green kingdom: chaos or self-order? Journal of Cell Science, 115(7), pp.1345-1354.

Wasteneys, G.O. \& Ambrose, J.C., 2009. Spatial organization of plant cortical microtubules: close encounters of the 2D kind. Trends in Cell Biology, 19(2), pp.62-71.

Wickett, N.J. et al., 2014. Phylotranscriptomic analysis of the origin and early diversification of land plants. Proceedings of the National Academy of Sciences of the United States of America, 111(45), pp.4859-4868.

Wightman, R. et al., 2013. SPIRAL2 determines plant microtubule organization by modulating microtubule severing. Current Biology, 23(19), pp.1902-1907.

Wu, S.-Z. \& Bezanilla, M., 2014. Myosin VIII associates with microtubule ends and together with actin plays a role in guiding plant cell division. eLife, 3, e03498.

Xu, B. et al., 2014. Contribution of NAC transcription factors to plant adaptation to land. Science, 343(6178), pp.1505-1508.

$\mathrm{Xu}$, X.M. et al., 2008. RanGAP1 is a continuous marker of the Arabidopsis cell division plane. Proceedings of the National Academy of Sciences of the United States of America, 105(47), pp.18637-18642.

Yamada, M. \& Goshima, G., 2017. Mitotic spindle assembly in land plants: Molecules and mechanisms. Biology (Basel), 6(1).

Yamada, M., Miki, T. \& Goshima, G., 2016. Imaging mitosis in the moss Physcomitrella patens. In P. Chang \& R. Ohi, eds. Methods in Molecular Biology (Clifton, N.J.). New York, NY, United States: Springer New York, pp. 263-282.

Yasuhara, H. \& Shibaoka, H., 2000. Inhibition of cell-plate formation by brefeldin A inhibited the depolymerization of microtubules in the central region of the phragmoplast. Plant \& Cell Physiology, 41(3), pp.300-310.

Yasuhara, H., Sonobe, S. \& Shibaoka, H., 1993. Effects of taxol on the development of the cell plate and of the phragmoplast in tobacco BY-2 cells. Plant \& Cell Physiology, 34(1), pp.21-29.

Zeng, C.J.T., Lee, Y.-R.J. \& Liu, B., 2009. The WD40 repeat protein NEDD1 functions in microtubule organization during cell division in Arabidopsis thaliana. The Plant Cell, 21(4), pp.1129-1140. 


\section{Abstract}

Different from animal cells that divide by constriction of the cortex inward, cells of land plants divide by initiating a new cell-wall segment from their center. For this, a disk-shaped, membrane-enclosed precursor termed the cell plate is formed that radially expands toward the parental cell wall (Staehelin \& Hepler 1996; Boruc \& van Damme 2015; Müller \& Jürgens 2015). The synthesis of the plate starts with the fusion of vesicles into a tubulo-vesicular network (Schmiedel et al. 1981; Samuels et al. 1995; Gu \& Verma 1996). Vesicles are putatively delivered to the division plane by transport along microtubules of the bipolar phragmoplast network that guides plate assembly (Euteneuer \& Mcintosh 1980; Otegui et al. 2001; Lee et al. 2001). How vesicle immobilization and fusion are then locally triggered is unclear. In general, a framework for how the cytoskeleton spatially defines cell-plate formation is lacking. Here we show that membranous material for cell-plate formation initially accumulates along regions of microtubule overlap in the phragmoplast of the moss Physcomitrella patens. Kinesin-4-mediated shortening of these overlaps at the onset of cytokinesis proved to be required to spatially confine membrane accumulation. Without shortening, the wider cell-plate membrane depositions evolved into cell walls that were thick and irregularly shaped. Phragmoplast assembly thus provides a regular lattice of short overlaps on which a new cell-wall segment can be scaffolded. Since similar patterns of overlaps form in central spindles of animal cells, involving the activity of orthologous proteins (Kurasawa et al. 2004; Hu et al. 2011), we anticipate that our results will help uncover universal features underlying membrane-cytoskeleton coordination during cytokinesis.

\section{Results and discussion}

Early ultrastructural work in the flowering plant Haemanthus katherinae has revealed that microtubules from the two halves of the phragmoplast interdigitate at the midplane where cell-plate vesicles aggregate (Hepler \& Jackson 1968). The same configuration exists in the phragmoplast of the moss Physcomitrella patens (Hiwatashi et al. 2014), indicating that it might be universally relevant for the spatial orchestration of cytokinesis in land plants. We therefore investigated whether regions of antiparallel microtubule overlap could have a direct role in determining the location of cell-plate assembly in the genetically amenable moss $P$. patens (Figure 1A). For this, we imaged the conserved microtubule bundling protein MAP65c labelled with citrine (a GFP variant) as a marker for microtubule overlaps (Müller et al. 2004; Gaillard et al. 2008; Kosetsu et al. 2013) in combination with the lipophilic dye FM4-64 that stained the membrane of the forming cell plate in life caulonema apical cells (Figure 1A-B). Membranous material first became visible in the phragmoplast midplane approximately 2 min after the onset of anaphase $(t=0)$. At this stage, cell-plate membrane and overlap signals were momentarily strongly correlated and appeared as two coinciding punctate patterns at the midplane (Figure 1B). As the construction of the cell plate progressed, membranous material also became visible in between microtubule overlaps. Later, the initial microtubule overlaps disappeared, leaving behind a continuous plane of membranous material. Throughout cytokinesis, new overlaps showing isolated membrane association were formed at the phragmoplast periphery, expanding the structure radially (Murata et al. 2013). It thus appeared that overlaps are platforms for the initial deposition of cell-plate membrane at the start of cytokinesis and thereafter at the expanding edge of the phragmoplast. This warranted further investigation of their dynamics throughout cell division. 


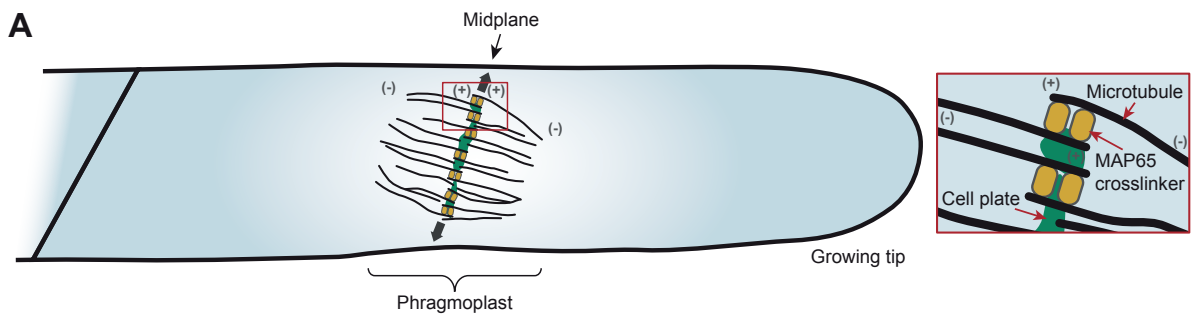

B

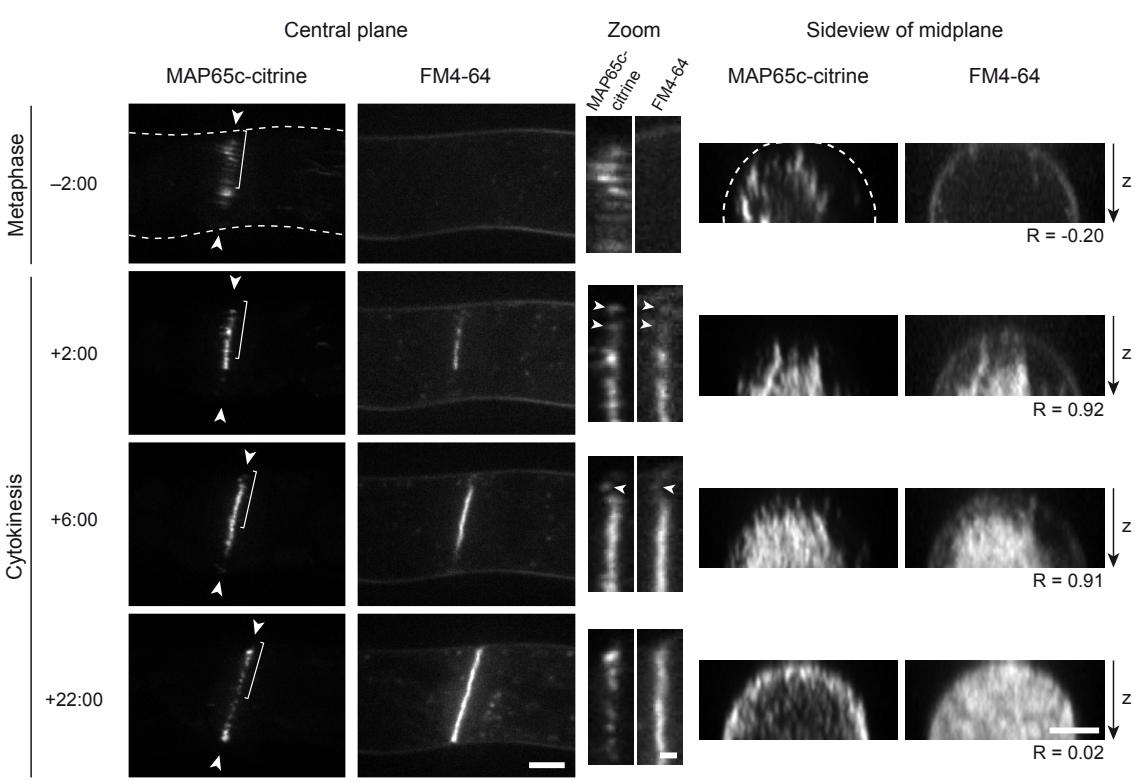

Figure 1 - Early cytokinetic membrane accumulation occurs at sites of microtubule overlap. (A) Schematic representation of cell-plate formation in a dividing $P$. patens caulonemal tip cell and definition of the terminology used. MAP65 resides in between interdigitating microtubules in the phragmoplast midplane. An enlarged view of the boxed area is shown on the right. Arrows indicate direction of radial expansion. Figure is not drawn to scale. (B) Snapshots of a dividing caulonemal tip cell expressing MAP65c-citrine stained with FM4-64 membrane dye. A single confocal plane is depicted. Time with respect to anaphase onset $(t=0)$ is indicated in min:s. Dashed lines indicate the cell boundary and arrowheads mark the midplane along which the depicted side views were generated. Magnified views of the areas indicated with the square brackets are shown. Here, the arrowheads highlight the punctate cell-plate membrane accumulations that colocalized with a MAP65-labeled region of overlap. The Pearson correlation coefficient $(R)$ between the MAP65 and FM4-64 signal intensities within the midplane is indicated on the right. Scale bars in main panels, $5 \mu \mathrm{m}$; scale bars in magnified panels, $1 \mu \mathrm{m}$.

At anaphase onset, overlap regions of interpolar microtubules of the spindle apparatus decreased in length and closely coordinated their mutual positions onto a straight plane perpendicular to the spindle axis (Figure 1B). This shortening of overlaps has likewise been reported in higher plants, and their limited length is maintained throughout cytokinesis (Müller et al. 2004; Ho et al. 2011). To investigate whether length confinement of overlaps in the phragmoplast is a prerequisite to precisely define the position of membrane deposition, we sought a way to interfere with overlap length. To this end, we investigated the functioning of kinesin- 4 motor proteins, whose disruption causes a marked increase in overlap length in central spindles of animal cells, but 
for which no cytokinetic function has yet been attributed in plants (Kurasawa et al. 2004; Hu et al. 2011; Kong et al. 2015). Two P. patens kinesin-4 molecules, Kinesin4-Ia and Kinesin4-Ic (hereafter referred to as Kin4-Ia and Kin4-Ic), were observed at the phragmoplast midplane in a $P$. patens localization screen (Miki et al. 2014). In our investigation, cells lacking both these kinesin-4 motors failed to shorten their overlaps at the start of anaphase and instead maintained long overlaps until the completion of cytokinesis (Figures 2A and S1). Smaller defects present in single mutants became particularly apparent when an average kymograph was constructed summarizing the overlap dynamics of many cells (Figure 2B). Cells that only lacked Kin4-Ia showed mild extensions during late cytokinesis, while overlap shortening was markedly delayed upon the sole removal of Kin4-Ic (Figure 2B, arrowheads). Interestingly, this delay corresponded in time with the late recruitment of Kin4-Ia onto cytokinetic overlaps (Figure S2A-B). Arrival

A

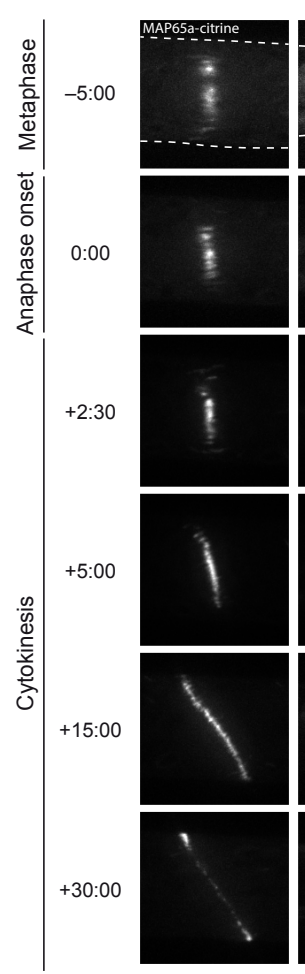

B

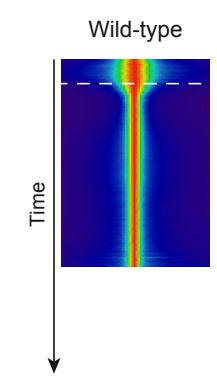

\section{$\Delta k i n 4-l a$}
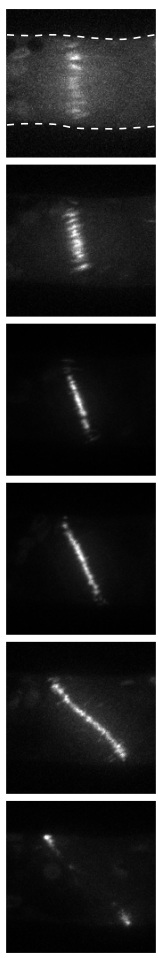

$$
\Delta \text { kin4-la }
$$

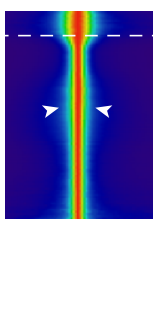

$\Delta k i n 4-/ c$
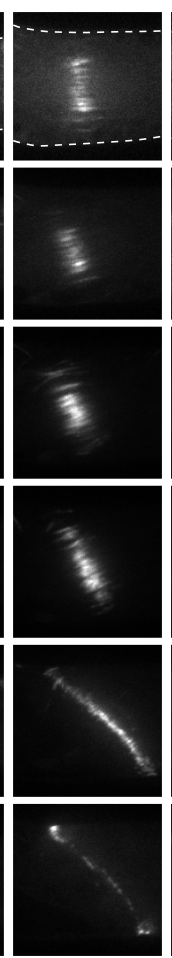

$\Delta$ kin4-la / $\Delta k i n 4-l c$
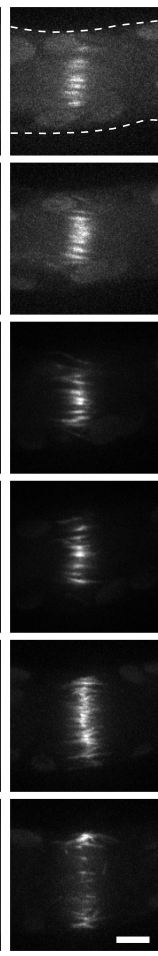

$\Delta$ kin4-la / $\Delta$ kin4-Ic

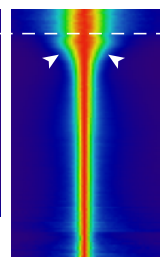

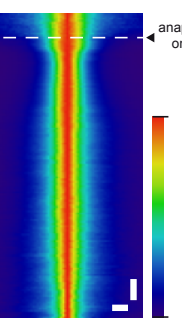

Figure 2 - Two kinesin-4s jointly limit microtubule overlap length in the phragmoplast midplane (A) Caulonemal tip cells expressing MAP65a-citrine and lacking Kin4-la and/or Kin4-Ic imaged throughout cell division. Dashed lines indicate the cell boundary. The time with respect to anaphase onset $(t=0)$ is indicated in min:s on the left. Images are maximum z-projections of three confocal planes spaced $0.5 \mu \mathrm{m}$ apart. Scale bar, $5 \mu \mathrm{m}$. See also Figures $\mathrm{S} 1$ and S2. (B) Evolution of the width of MAP65a-labeled overlaps throughout cell division shown by a kymograph for wild-type (average of $n=7$ cells), $\Delta$ kin4la $(\mathrm{n}=9), \Delta k i n 4-I c(\mathrm{n}=9)$, and $\Delta k i n 4-l a / \Delta k i n 4-l c(\mathrm{n}=9)$. Per time point, the normalized intensity distribution perpendicular to the midplane is shown after undulations in the midplane were straightened out computationally. Kymographs were limited to contain only information from at least half of the imaged cells. Arrowheads mark the slight overlap extension in the $\Delta$ kin4-la mutant and the delay in overlap compaction after anaphase onset in the $\Delta k i n 4-I c$ mutant. Horizontal scale bar, $1 \mu \mathrm{m}$; vertical scale bar, 5 min. 
of either motor was thus sufficient for overlap shortening. In addition to extended overlaps, we noted an absence of the characteristic rotation of the division plane to an oblique orientation in the kinesin-4 double mutant (Figure 2A). Consequently, finalized cross walls in this mutant ran primarily perpendicular to the cell axis (Figure S2C-D). Whether kinesin-4 has a direct role in division plane reorientation is currently unclear because the underlying process is mechanistically not understood.

The mechanism by which kinesin- 4 induces overlap shortening was investigated using a purified recombinant $\mathrm{N}$-terminal fragment of moss Kin4-Ic. We observed that it suppressed microtubule growth velocities in a dose-dependent manner with a minimal effect on catastrophe rate (Figure S2E-H). The mode of action of kinesin- 4 thus appeared to be conserved between animals and plants (Bringmann et al. 2004; Bieling et al. 2010; van der Vaart et al. 2013). Recruitment of kinesin-4 onto phragmoplast overlaps may thus prevent their elongation by inhibiting the growth of microtubules within them. Shortening could then occur if microtubules simultaneously slide apart (Kurasawa et al. 2004; Hu et al. 2011; Bieling et al. 2010).

Having found a way to increase overlap length, we next examined the consequences for the accumulation of cell-plate membrane. The practical usability of FM4-64 to visualize cell-plate membranes was limited because staining, although bright, was variable and dye administration required precise timing, hampering quantitative comparison across genotypes. We therefore labelled an endogenous integral membrane protein belonging to the secretory carrier membrane protein (SCAMP) family, which we designated SCAMP4 (Figure S3A-C). This protein localized to the plasma membrane, early endosomal compartments and the cell plate as described for SCAMPs of higher plants (Law et al. 2012) (Figure S3D-E). We first inspected the expanding edge of the phragmoplast where, as described above, cell-plate material specifically accumulated at overlaps (Figure 1B). Compared to wild-type cells, longer membrane structures were visible along the extended overlaps of the $\Delta k i n 4-I a / I c$ mutant but rarely did they encompass the entire overlap length (Figure 3A). Upon a closer inspection of the dynamics of the assembly process, we noted that particularly long stretches of membrane material did occasionally form (Figure $3 \mathrm{E})$. However, these readily coalesced into the cell plate even while the underlying overlaps were still intact. To assess whether this remodeling could eventually reduce the width of the cell plate to a wild-type level, we next analyzed more mature parts of the cell plate where membrane material was also visible in between overlaps (Figure 3B). In the $\Delta k i n 4-I a / I c$ mutant, membrane material clearly aggregated near the centre of overlaps, but an overall widening of the cell plate remained notable (Figure 3B). Differences were verified by averaging line scans of SCAMP4 signals and determining the widths of their intensity distribution (Figures 3C-D and S4A). A consistent widening of the cell plate in the kinesin-4 double mutant was also visible using differential interference contrast (DIC) optics (Figure S4B-C). Taken together, confinement of the initial deposition of cell-plate membrane through kinesin-4-mediated overlap length restriction is essential to control ultimate cell-plate thickness.

Apart from the observed broadening of cell plates, we also noticed a delayed progression of cytokinesis in cells lacking both kinesin-4s. Compared to wild-type cells, the radial expansion velocity of the phragmoplast was halved in kinesin-4 double-mutant cells (Figure 3F). Since expansion requires the continuous formation of new microtubule overlaps at the phragmoplast edge (Murata et al. 2013), we hypothesized that kinesin-4 may have had a direct role in the formation of new overlaps. However, abundant overlap formation during expansion was still discernible in the mutant situation (See movie S4 in de Keijzer et al. 2017). Moreover, 
A

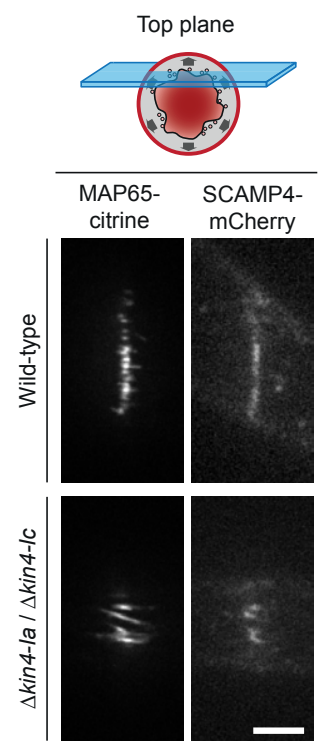

C

Top plane membrane distribution profile

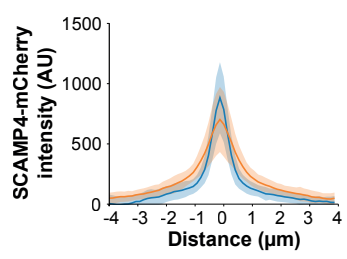

B

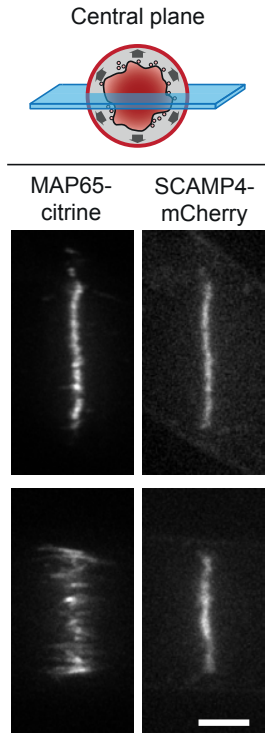

D
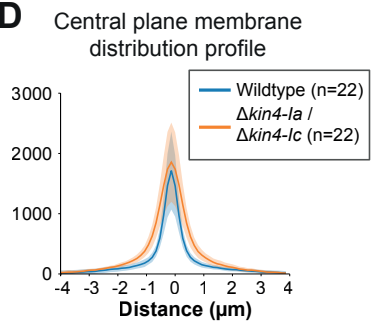

E
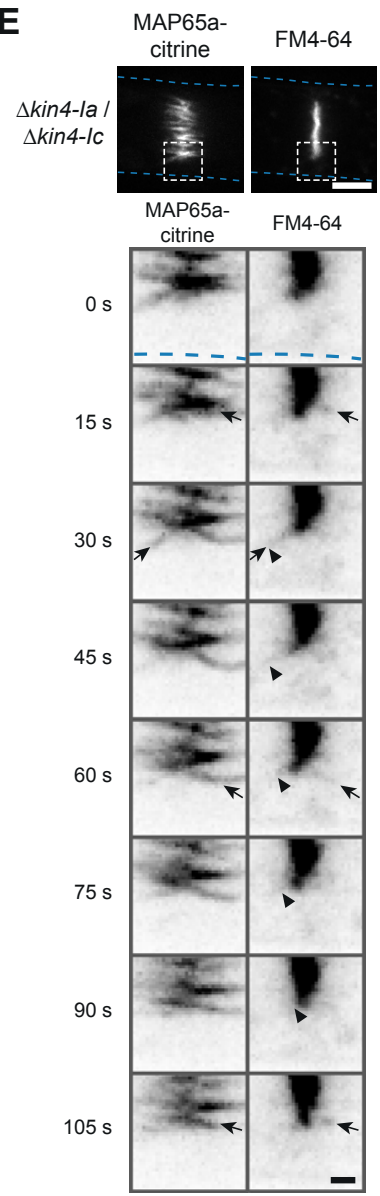

G

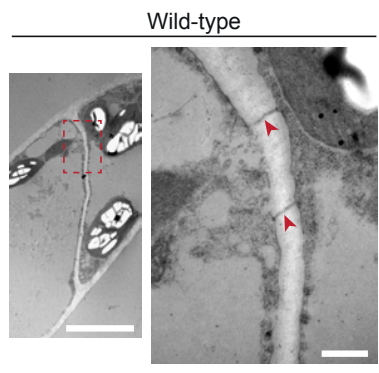

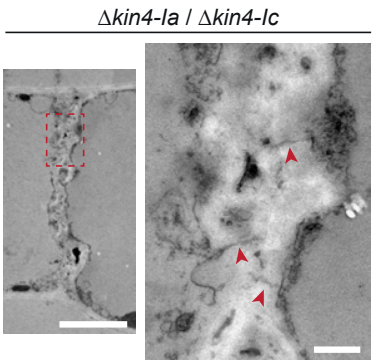

Figure 3 - Broadened membrane deposition upon overlap overextension hampers cellplate assembly. (A) Early membrane accumulation analyzed in single confocal planes acquired at the phragmoplast expansion site for dividing wild-type and $\Delta k i n 4-l a / \Delta k i n 4-l c$ cells. MAP65-labeled overlaps and cell-plate membranes visualized using SCAMP4-mCherry are shown. The location of the depicted confocal planes is illustrated at the top. Scale bar, 5 $\mu \mathrm{m}$. See also Figure S3. (B) Cell-plate membrane distribution analyzed in the central plane corresponding to a site of more advanced cell-plate maturation, visualized as described in (A). (C) Spatial distribution profile of early cell-plate membrane accumulation measured perpendicular to the division plane using the average SCAMP4-mCherry signal intensity in

(legend continued on next page) 
simultaneous imaging of Kin4-Ic and MAP65a demonstrated that overlaps became enriched with kinesin-4 only after they were formed (Figure S4D-E). We therefore favour an alternative scenario in which the expansion rate of the phragmoplast is coupled to the degree of maturation in the central part of the cell plate. The slower expansion in the kinesin- 4 double mutant may then be explained by the initial wider deposition of membrane material requiring more time to be transformed into a coherent cell plate.

As the cell-plate lumen becomes reinforced with various polysaccharides, it gradually matures into a new cell-wall segment (Samuels et al. 1995; Drakakaki 2015). The abnormalities in cellplate assembly arising from longer microtubule overlaps were thus expected to be reflected in the morphology of completed cross walls. Indeed, when observed using transmission electron microscopy, completed walls in kinesin-4 double mutants were irregularly shaped, thicker (1.32 $\pm 0.32 \mu \mathrm{m}[\mathrm{SD} ; \mathrm{n}=23]$ against $0.46 \pm 0.13 \mu \mathrm{m}[\mathrm{n}=27]$ in wild-type; $\mathrm{p}<0.001$ in Student's $t$-test) and showed an abnormal texture, often with visible inclusions (Figure 3G). Interestingly, mild structural defects were also observed in both kinesin-4 single mutants, highlighting that even a mild disruption of overlap length can ultimately result in improperly deposited walls (Figure S4F-G). It will be interesting to find out how the dwarfed phenotype of double-mutant plants relates to the defective interface between neighbouring cells (Figure S1C). A key linking factor might be the permeability of plasmodesmata, which is known to affect signalling and differential development among plant cells (Brunkard et al. 2015; Han \& Kim 2016). Channels of plasmodesmata appeared crooked in the kinesin-4 double mutant (Figure 3G, arrowheads). However, the consequence for intercellular transport remains to be investigated.

The classical view on cell-plate assembly is that vesicles are transported along microtubules toward the phragmoplast midplane (Staehelin \& Hepler 1996; Boruc \& van Damme 2015; Müller \& Jürgens 2015; Otegui et al. 2001; Lee et al. 2001). The polarized orientation of microtubules alone, however, does not explain why cell-plate initials are only formed there. With our current findings, we propose a leading role for overlaps in spatially orchestrating vesicle immobilization and facilitating their localized fusion (Figure 4). How cell-plate vesicles can become associated to these specific cytoskeletal structures is then a relevant question. We reasoned that vesicle transport may stall at overlaps if vesicle-associated motor proteins interact with microtubules of opposite polarity (Derivery et al. 2015). Alternatively, vesicles may diffuse passively and bind overlap-associated factors to accumulate locally. In both scenarios, the successive step of vesicle fusion possibly requires the recruitment of vesicle tethering and fusion machinery onto overlaps (Figure 4) (Fendrych et al. 2010; Rybak et al. 2014; Boruc \& van Damme 2015; Steiner et al.

wild-type and $\Delta k i n 4-l a / \Delta k i n 4-l c$ cells. The peak intensities were centered at $0 \mu \mathrm{m}$ for individual cells before averaging. See also Figure S4A-C. (D) Distribution of cell-plate membranes in the central plane measured as described in (C). (E) Dynamics of membrane remodeling at overlap sites visualized in a $\Delta$ kin4-la/ $\Delta$ kin4-IC mutant cell expressing MAP65a-citrine and stained with FM4-64 to label membranes of the cell plate. A detailed view of the boxed area is shown in inverted contrast. Arrows point to elongated stretches of membrane associated with overlaps. The arrowheads point to an incident in which a stretch clearly coalesced into the forming cell plate. Images are maximum z-projections of two confocal planes spaced 0.5 $\mu \mathrm{m}$ apart. Scale bar, $5 \mu \mathrm{m}$ in the overview image and $1 \mu \mathrm{m}$ in the enlarged views. (F) Radial phragmoplast expansion velocities in wild-type and kinesin-4-deficient cells. Individual measurements for $n=10$ cells and their medians are shown. ${ }^{* *}$ Differences supported with $p$ $<0.001$ in Student's $t$ test. See also Figure S4D-E. (G) Transmission electron micrographs of cross walls in wild-type and the $\Delta$ kin4-la/ $\Delta$ kin4-Ic mutant. An enlarged view of the boxed areas is shown on the right. Arrowheads indicate plasmodesmata, which appear crooked in the mutant cell wall. Scale bars, $5 \mu \mathrm{m}$ in the left images and $500 \mathrm{~nm}$ in the enlarged views. 
2016). It is highly conceivable that MAP65 plays a direct role in this based on recruitment strategies in related bipolar microtubule networks (Lee et al. 2012; Duellberg et al. 2013). Such a notion is also congruent with silencing of MAP65 in moss upon which membrane accumulation stalls during early cytokinesis and no uniform cell plate is formed (Kosetsu et al. 2013). We anticipate that the kinesin- 4 mutants will be instrumental to further identify and understand the signalling pathways operating at the phragmoplast midplane.

P. patens WT situation

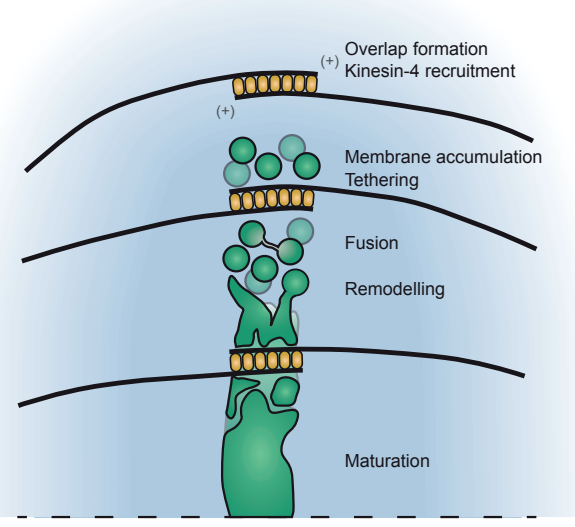

P. patens $\Delta$ kinesin-4 situation

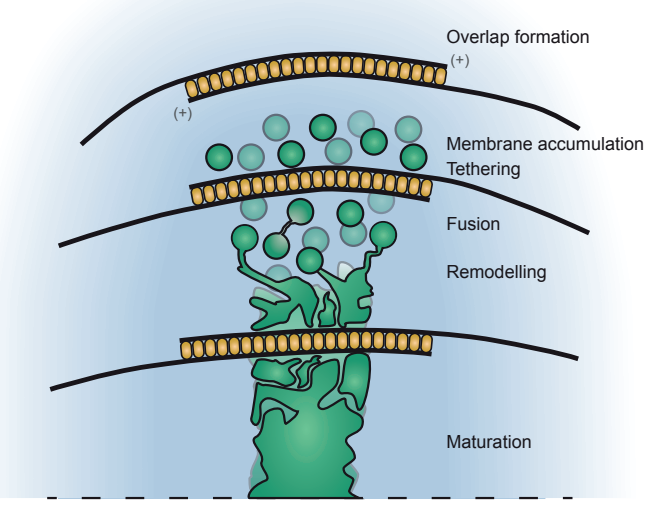

Figure 4 - Diagram of the successive steps in cell-plate assembly and disruption thereof upon kinesin-4 removal. Under wild-type circumstances (left), after the formation of a region of antiparallel overlap involving MAP65 crosslinkers (yellow), kinesin-4 is recruited to limit overlap length. This in turn confines the initial cell-plate membrane deposition to a well-defined plane. From our light microscopic observations, it is unclear whether the transformation of vesicles into larger membrane structures, involving tethering and fusion, occurs at overlaps or in their direct vicinity. Initial membrane structures are remodeled, and further maturation of the cell plate is achieved through deposition of cell-wall polysaccharides (Gu \& Verma, 1996; Drakakaki, 2015). In absence of kinesin-4 inhibitors of microtubule growth (right), elongated overlaps widen the zone of initial membrane deposition. Compared to wild-type, this wide deposition is remodeled into a thicker cell plate at a greatly impaired rate.

For long it has been recognized that regions of microtubule overlap in animal central spindles and plant phragmoplasts form a highly regular lattice during cytokinesis (Euteneuer \& Mcintosh 1980; Otegui et al. 2005). Our results show that overlaps in an early divergent land plant are fine-tuned this way to precisely define a plane on which the cell plate is scaffolded. Overlaps therefore not only have a structural role in maintaining the bipolarity of the phragmoplast microtubule network (Janson et al. 2007; Gaillard et al. 2008; Subramanian et al. 2010; Ho et al. 2011; Kosetsu et al. 2013; de Keijzer et al. 2014), but provide a well-defined spatial signal for cell-plate membrane aggregation and subsequent fusion. In dividing animal cells, microtubule overlaps of the central spindle concentrate signalling molecules to locally promote contractile ring formation and abscission (Wheatley \& Wang 1996; Lekomtsev et al. 2012; Green et al. 2012). A high degree of overlap organization therefore seems to be a common requirement for localizing cytokinetic machinery, and the involvement of multiple conserved factors including MAP65 and kinesin-4 suggests that its necessity arose early in the evolution of the eukaryotic cell. The identification of overlaps as central players in phragmoplast functioning therefore does not only provide a framework for future work on plant cytokinesis, but provides means to discover common mechanisms in diverse cytokinetic networks that all coordinate cytoskeletal and membrane-based processes. 


\section{Materials and methods}

\section{Plasmids and cloning procedures}

All plasmids used were generated using standard restriction-ligation cloning procedures and were verified by sequencing. The use of plasmids and primers is summarized in tables 1 and 2 below.

Table 1 - Primers used in this study

\begin{tabular}{|c|c|c|c|}
\hline Primer & Use & Sequence $\left(5^{\prime}>3^{\prime}\right)$ & RE site \\
\hline TM227 & $\begin{array}{l}\text { Cloning of GFP/mCherry fluoro- } \\
\text { phore }\end{array}$ & $\begin{array}{l}\text { ATAAGCTTGATATCTTGGTGAGCAAGGGCGAG- } \\
\text { GAGCTG }\end{array}$ & HindllI \\
\hline JK74 & $\begin{array}{l}\text { Cloning of GFP/mCherry fluoro- } \\
\text { phore }\end{array}$ & AGGAATTCTTACTTGTACAGCTCGTCCATGC & EcoRI \\
\hline JK117 & $\begin{array}{l}\text { Cloning of nopaline synthase } \\
\text { terminator }\end{array}$ & GCGAATTCCTACAGCCCCGAAATAAC & EcoRI \\
\hline JK118 & $\begin{array}{l}\text { Cloning of nopaline synthase } \\
\text { terminator }\end{array}$ & AACTGCAGCTCATGTTTGACAGCTTATCATCGG & Pstl \\
\hline JK138 & $\begin{array}{l}\text { Cloning of SCAMP4 left targeting } \\
\text { arm }\end{array}$ & CCGCTCGAGGCCAAAACATGGAAACCTACCTTAGG & Xhol \\
\hline JK139 & $\begin{array}{l}\text { Cloning of SCAMP4 left targeting } \\
\text { arm }\end{array}$ & CCCAAGCTTGATAGCTACTCTGCTTGCTGCAGC & HindIII \\
\hline JK140 & $\begin{array}{l}\text { Cloning of SCAMP4 right targeting } \\
\text { arm }\end{array}$ & GCTCTAGATTTTACTGTATTCTTATTGTTAGTTGAGG & Xbal \\
\hline JK141 & $\begin{array}{l}\text { Cloning of SCAMP4 right targeting } \\
\text { arm }\end{array}$ & CGCGAGCTCCATCATACCACTTCTCAAACACATGC & Sacl \\
\hline $\begin{array}{l}\text { Kin4-Ic-5 } \\
\text { UTR Fw }\end{array}$ & $\begin{array}{l}\text { Cloning of Kin4-Ic KO left target- } \\
\text { ing arm }\end{array}$ & AAAGGTACCGCCAAGGAGCAAAGATACACGGCTG & Kpnl \\
\hline $\begin{array}{l}\text { Kin4-Ic-5 } \\
\text { UTR Rv }\end{array}$ & $\begin{array}{l}\text { Cloning of Kin4-Ic KO left target- } \\
\text { ing arm }\end{array}$ & AAACTCGAGCGTCGCTACTAATGTACACCCAGC & Xhol \\
\hline $\begin{array}{l}\text { Kin4-Ic-3' } \\
\text { UTR Fw }\end{array}$ & $\begin{array}{l}\text { Cloning of Kin4-Ic KO right target- } \\
\text { ing arm }\end{array}$ & AAACATATGCATTTGTGGGGTTCAACCTGAGC & Ndel \\
\hline $\begin{array}{l}\text { Kin4-Ic-3' } \\
\text { UTR Rv }\end{array}$ & $\begin{array}{l}\text { Cloning of Kin4-lc KO right target- } \\
\text { ing arm }\end{array}$ & AAACCGCGGGAAGATTCAAAAAGGTTAGC & Sacll \\
\hline JK198 & $\begin{array}{l}\text { Cloning of Kin4-la KO left target- } \\
\text { ing arm }\end{array}$ & GGGGTACCTGAAATCGAGATATGGCAGCAGC & Kpnl \\
\hline JK199 & $\begin{array}{l}\text { Cloning of Kin4-la KO left target- } \\
\text { ing arm }\end{array}$ & CCGTCGAGTTGTGCACTGATTTGTGAATCAAGTG & Xhol \\
\hline JK234 & Cloning of Kin4-Ic motor domain & CATGCCATGGGAGCAGACAAAGCAAACG & Ncol \\
\hline JK235 & Cloning of Kin4-Ic motor domain & $\begin{array}{l}\text { AGAAGCGGCCGCTCCGTTAGGAGCAGTATCTTTA- } \\
\text { GACG }\end{array}$ & Notl \\
\hline JK220 & $\begin{array}{l}\text { SCAMP4 GFP/mCherry tagging } \\
\text { genotyping }\end{array}$ & GTGATCAACGTGGGATTAAAGAAGTCAC & - \\
\hline JK206 & Kin4-la deletion genotyping & TTTGAGGTGTATCACCCAGTTTCG & - \\
\hline JK207 & Kin4-la citrine tagging genotyping & GGAGGAAAGTGATGGCGAAAGAGC & - \\
\hline JK208 & $\begin{array}{l}\text { Kin4-la deletion / citrine tagging } \\
\text { genotyping }\end{array}$ & GCAGGAAGCACGAGCTAAGACC & - \\
\hline JK221 & $\begin{array}{l}\text { Kin4-la gene specific primer - } \\
\text { begin }\end{array}$ & GTTGAGACGCTGAAGGACAGTGC & - \\
\hline JK222 & $\begin{array}{l}\text { Kin4-la gene specific primer - } \\
\text { begin }\end{array}$ & CCAGCCAAGTCAACAAGGTGAAGC & - \\
\hline JK223 & Kin4-la gene specific primer - end & GCAGAATTGGTTGGAACAGGAGC & - \\
\hline JK224 & Kin4-la gene specific primer - end & TCCACATCTCGCCTCTTCACAGC & - \\
\hline JK209 & Kin4-Ic deletion genotyping & CAGAAAGTGCCGAATGCGCTCC & - \\
\hline JK210 & Kin4-Ic citrine tagging genotyping & ACTGACCTGCCACATTCCGAGC & - \\
\hline JK211 & $\begin{array}{l}\text { Kin4-Ic deletion / citrine tagging } \\
\text { genotyping }\end{array}$ & TTGACTTGTGTGGAATCTTGGATGC & - \\
\hline JK212 & $\begin{array}{l}\text { Kin4-Ic gene specific primer - } \\
\text { begin }\end{array}$ & ССТACCACAATGGGCAAACCTCC & - \\
\hline
\end{tabular}


Table 1 - Primers used in this study

\begin{tabular}{|c|c|c|c|}
\hline Primer & Use & Sequence $\left(5^{\prime}>3^{\prime}\right)$ & RE site \\
\hline JK213 & $\begin{array}{l}\text { Kin4-Ic gene specific primer - } \\
\text { begin }\end{array}$ & GCGTTTGAGTCTGCTGGGCTTAC & - \\
\hline JK214 & Kin4-Ic gene specific primer - end & GACCCTCTTGCACCTCATGTTTGG & - \\
\hline JK215 & Kin4-Ic gene specific primer - end & GAATGTGGCAGGTCAGTGCTCG & - \\
\hline JK130 & $\begin{array}{l}\text { Primer on resistance cassettes } \\
\text { forward }\end{array}$ & TGCTAAGGCAGGGTTGGTTACG & - \\
\hline JK160 & $\begin{array}{l}\text { Primer on resistance cassettes } \\
\text { reverse }\end{array}$ & TGCGAAGGATAGTGGGATTGTGC & - \\
\hline JK125 & $\begin{array}{l}\text { Sequencing of terminator after } \\
\text { mCherry }\end{array}$ & AGAGGCTGAAGCTGAAGGACG & - \\
\hline JK129 & $\begin{array}{l}\text { Sequencing region upstream of } \\
\text { mCherry }\end{array}$ & CCCTTGGTCACCTTCAGCTTGG & - \\
\hline JK112 & $\begin{array}{l}\text { Sequencing region upstream of } \\
\text { GFP }\end{array}$ & GCCGGACACGCTGAACTTGTG & - \\
\hline JK114 & $\begin{array}{l}\text { Sequence downstream of resi- } \\
\text { sistance cassette }\end{array}$ & ACTCAAGAGGATAAAACCTCACC & - \\
\hline JK111 & Construct sequencing general & GATTAAGTTGGGTAACGCCAGG & - \\
\hline JK116 & Construct sequencing general & ACACAGGAAACAGCTATGACC & - \\
\hline JK236 & $\begin{array}{l}\text { Sequencing of pET28a-Kin4- } \\
\text { Ic-(794) }\end{array}$ & GCGAAATTAATACGACTCACTATAGGG & - \\
\hline JK237 & $\begin{array}{l}\text { Sequencing of pET28a-Kin4- } \\
\text { Ic-(794) }\end{array}$ & GCTAGTTATTGCTCAGCGGTGG & - \\
\hline JK238 & $\begin{array}{l}\text { Sequencing of pET28a-Kin4- } \\
\text { Ic-(794) }\end{array}$ & CTACCACAATGGGCAAACCTCC & - \\
\hline JK239 & $\begin{array}{l}\text { Sequencing of pET28a-Kin4- } \\
\text { Ic-(794) }\end{array}$ & AGCCTACGGTCAATCGAGATCC & - \\
\hline
\end{tabular}

Table 2 - Plasmids used in this study

\begin{tabular}{|c|c|c|}
\hline Plasmid & Usage & Source \\
\hline pTM374 & C-terminal citrine tagging of Kin4-la & (Miki et al. 2014) \\
\hline pTM365 & C-terminal citrine tagging of Kin4-Ic & (Miki et al. 2014) \\
\hline pmCherry-LoxP-BsdR & $\begin{array}{l}\text { Vector for making c-terminal mCherry fusion pro- } \\
\text { teins at an endogenous locus; Blasticidin resistance }\end{array}$ & This study \\
\hline peGFP-nptll & $\begin{array}{l}\text { Vector for making c-terminal GFP fusion proteins at } \\
\text { an endogenous locus; G418 resistance }\end{array}$ & This study \\
\hline SCAMP4-mCherry & C-terminal mCherry tagging of SCAMP4 & This study \\
\hline SCAMP4-GFP & C-terminal GFP tagging of SCAMP4 & This study \\
\hline Kin4-Ic-KO-HygR & Knock-out of the entire Kin4-Ic reading frame & This study \\
\hline Kin4-la-KO-HygR & $\begin{array}{l}\text { Knock-out of the entire Kin4-la reading frame; } \\
\text { Hygromycin resistance }\end{array}$ & This study \\
\hline Kin4-la-KO-NatR & $\begin{array}{l}\text { Knock-out of the entire Kin4-la reading frame; Nour- } \\
\text { seothricin resistance }\end{array}$ & This study \\
\hline MAP65a-mCherry & C-terminal mCherry tagging of MAP65a & This study \\
\hline pET28a-Kin4-Ic-(794) & Bacterial production of $\mathrm{N}$-terminal Kin4-Ic fragment & This study \\
\hline
\end{tabular}

pmCherry-LoxP-BsdR

First, the sequence encoding the citrine fluorophore in pCTRN-nptII (Hiwatashi et al. 2008) was exchanged for that of mCherry using a fragment amplified with primers TM227 and JK74 and introduced using HindIII and EcoRI sites. Next, the nopaline synthase terminator (Tnos) and 
nptII resistance cassette were removed using BamHI and EcoRI. Finally, the blasticidin resistance cassette, obtained from p35S-loxP-BsdR (AB537973) as a PstI / BamHI fragment, and Tnos, obtained as a PCR fragment amplified from pCTRN-nptII using JK117 and JK118 and digested with EcoRI and PstI, were introduced.

\section{peGFP-nptII}

The citrine fluorophore encoding sequence in pCTRN-nptII was exchanged for that of eGFP amplified with primers TM227 and JK74 using HindIII and EcoRI sites.

\section{SCAMP4-mCherry}

A $\sim 0.7 \mathrm{~kb}$ region of the 3 ' end of the SCAMP4 gene and a $\sim 1.1 \mathrm{~kb}$ region downstream of the stop codon were amplified using primers JK138 till JK141 and cloned into pmCherry-LoxPBsdR using XhoI / HindIII and XbaI / SacI sites.

\section{SCAMP4-GFP}

The mCherry fluorophore coding sequence and blasticidin resistance cassette were removed from SCAMP4-mCherry and replaced with eGFP and the nptII resistance cassette obtained from peGFP-nptII using HindIII and XbaI sites.

\section{$\underline{\mathrm{Kin} 4-\mathrm{Ic}-\mathrm{KO}-\mathrm{Hyg} \mathrm{R}}$}

$\mathrm{A} \sim 0.8 \mathrm{~kb}$ region upstream the initiation codon of the Kin4-Ic gene and a $\sim 1.0 \mathrm{~kb}$ region downstream of the stop codon were amplified using primers Kin4-Ic-5' UTR Fw, Kin4-Ic-5' UTR Rv, Kin4-Ic-3' UTR Fw and Kin4-Ic-3' UTR Rv and cloned into p35S-loxP-BsdR (AB537973) using $K p n \mathrm{I} / \mathrm{XhoI}$ and NdeI / SacII sites. The blasticidin resistance cassette was subsequently replaced with the hygromycin resistance cassette from p35S-LoxP-HygR (p'TN186) using XhoI and $X b a \mathrm{I}$.

\section{Kin4-Ia-KO-HygR}

The $\sim 1.0 \mathrm{~kb}$ region after the stop codon of Kin4-Ia was obtained from pTM374 as a BamHI / SacI fragment and inserted into p35S-LoxP-HygR (pTN186). A 1.0 kb region upstream of the start codon, amplified using primers JK198 and JK199 was subsequently introduced using $K p n \mathrm{I}$ and $\mathrm{XhoI}$.

\section{$\underline{\text { Kin4-Ia-KO-NatR }}$}

The hygromycin resistance cassette of Kin4-Ia-KO-HygR was replaced with the nourseothricin resistance cassette from the $\gamma$-tubulin-a-KO plasmid (Nakaoka et al. 2015) using EcoRV, BamHI and SacI.

\section{MAP65a-mCherry}

The sequence encoding the citrine fluorophore and nptII resistance cassette in the MAP65acitrine plasmid (Kosetsu et al. 2013) was replaced with that encoding mCherry and the blasticidin resistance cassette from pmCherry-LoxP-BsdR using ClaI and NotI.

pET28a-Kin4-Ic-(794)

The sequence encoding the first 794 amino acids of Kin4-Ic was PCR amplified from a cDNA library generated from P. patens protonemal tissue using primers JK234 and JK235. The resulting product was digested with $\mathrm{NcoI}$ and $\mathrm{NotI}$ and subsequently ligated into pET28a.

\section{$\boldsymbol{P}$. patens growth conditions and transformation}

P. patens tissues were grown on BCDAT plates under continuous light, unless stated otherwise. Plasmids were linearized and introduced into the $P$. patens genome by homologous recombination 
using PEG-mediated protoplast transformation (Yamada et al. 2016). Correct insertion events were characterised by PCR. A summary of the characteristics of generated moss lines and their use throughout the study are listed in table 3 below. For imaging, protonemal tissue was grown on BCD medium in glass bottom dishes (Yamada et al. 2016).

\begin{tabular}{|c|c|c|c|c|c|c|}
\hline Name & Experimental use & $\begin{array}{l}\text { Main } \\
\text { clone }\end{array}$ & $\begin{array}{l}\text { Background } \\
\text { strain }\end{array}$ & $\begin{array}{l}\text { Plasmid } \\
\text { transformed }\end{array}$ & Figure & Source \\
\hline MAP65a-citrine & $\begin{array}{l}\text { Kinesin-4 knock-out con- } \\
\text { struction }\end{array}$ & \#2 & WT & see reference & $\begin{array}{l}2,3 F-G, S 1 \\
\text { S2C-D, S4B-C, } \\
\text { S4G }\end{array}$ & $\begin{array}{l}\text { (Kosetsu et } \\
\text { al. 2013) }\end{array}$ \\
\hline MAP65c-citrine & $\begin{array}{l}\text { Overlap / FM4-64 colocal- } \\
\text { ization }\end{array}$ & \#5 & WT & see reference & $1 \mathrm{~B}$ & $\begin{array}{l}\text { (Kosetsu et } \\
\text { al. 2013) }\end{array}$ \\
\hline$\Delta k i n 4-l a$ & $\begin{array}{l}\text { Measuring effect of Kine- } \\
\text { sin-4 removal on overlap } \\
\text { dimension }\end{array}$ & \#7 & MAP65a-citrine & Kin4-la-KO-HygR & $\begin{array}{l}2,3 F, S 1 \\
S 2 C-D, S 4 F-G\end{array}$ & This study \\
\hline$\Delta k i n 4-I c$ & $\begin{array}{l}\text { Measuring effect of Kine- } \\
\text { sin- } 4 \text { removal on overlap } \\
\text { dimension }\end{array}$ & \#172 & MAP65a-citrine & Kin4-Ic-KO-HygR & $\begin{array}{l}2,3 F, S 1 \\
\text { S2C-D, S4F-G }\end{array}$ & This study \\
\hline$\Delta k i n 4-l a ~ / ~ \Delta k i n 4-l c$ & $\begin{array}{l}\text { Measuring effect of Kine- } \\
\text { sin-4 removal on overlap } \\
\text { dimension }\end{array}$ & \#66 & $\Delta k i n 4-l c$ & Kin4-la-KO-NatR & $\begin{array}{l}\text { 2, 3E-G, S1, } \\
\text { S2C-D, S4B-C, } \\
\text { S4G }\end{array}$ & This study \\
\hline SCAMP4-GFP & $\begin{array}{l}\text { Validation of SCAMP4 } \\
\text { as cell plate membrane } \\
\text { marker }\end{array}$ & \#42 & WT & SCAMP4-mCherry & S3C-D & This study \\
\hline SCAMP4-mCh & $\begin{array}{l}\text { Cell plate membrane / } \\
\text { overlap colocalization }\end{array}$ & \#173 & MAP65c-citrine & SCAMP4-mCherry & $3 A-D, S 3 C, S 4 A$ & This study \\
\hline $\begin{array}{l}\Delta k i n 4-/ c \text { / } \\
\text { SCAMP4-mCh }\end{array}$ & $\begin{array}{l}\text { Generating Kinesin- } 4 \text { dou- } \\
\text { ble mutant with membrane } \\
\text { marker }\end{array}$ & $\# 44$ & $\Delta k i n 4-I c$ & SCAMP4-mCherry & S1A, S3C & This study \\
\hline $\begin{array}{l}\Delta k i n 4-l a ~ / ~ \Delta k i n 4-l c \\
\text { / SCAMP4-mCh }\end{array}$ & $\begin{array}{l}\text { Cell plate membrane / } \\
\text { overlap colocalization in } \\
\text { Kin- } 4 \text { double mutant }\end{array}$ & \#391 & $\begin{array}{l}\Delta k i n 4-l c / \\
\text { SCAMP4-mCh }\end{array}$ & Kin4-la-KO-NatR & $3 \mathrm{~A}-\mathrm{D}, \mathrm{S} 1 \mathrm{~A}, \mathrm{~S} 4 \mathrm{~A}$ & This study \\
\hline Kin4-la-citrine & $\begin{array}{l}\text { Intensity analysis of Kin4-la } \\
\text { throughout cytokinesis }\end{array}$ & $\# 43$ & WT & pTM374 & S1C, S2A-B & $\begin{array}{l}\text { This study; } \\
\text { (Miki et al. } \\
\text { 2014) }\end{array}$ \\
\hline Kin4-Ic-citrine & $\begin{array}{l}\text { Intensity analysis of Kin4-Ic } \\
\text { throughout cytokinesis }\end{array}$ & $\# 57$ & WT & pTM365 & S1C, S2A-B & $\begin{array}{l}\text { This study; } \\
\text { (Miki et al. } \\
\text { 2014) }\end{array}$ \\
\hline $\begin{array}{l}\text { Kin4-Ic-citrine / } \\
\text { MAP65a-mCh }\end{array}$ & $\begin{array}{l}\text { Kinesin-4 / overlap colo- } \\
\text { calization }\end{array}$ & \#140 & Kin4-Ic-citrine & MAP65a-mCh & S4D-E & This study \\
\hline
\end{tabular}

\section{Recombinant protein production}

An N-terminal, his-tagged fragment comprising the motor domain and coiled-coil regions required for dimerization of Kin4-Ic was produced in E. coli Rosetta2 (DE3) cells harbouring the pET28a-Kin4-Ic-(794) plasmid. Production was triggered through the addition of $0.2 \mathrm{mM}$ IPTG and performed at $18{ }^{\circ} \mathrm{C}$ for 20 hours. Cells were lysed using a French press in a phosphate buffer $\left(50 \mathrm{mM} \mathrm{NaH} \mathrm{PO}_{4}, 250 \mathrm{mM} \mathrm{NaCl}, 10 \% \mathrm{w} / \mathrm{v}\right.$ glycerol, $\left.\mathrm{pH} 7.4\right)$, containing a protease inhibitor cocktail (Roche diagnostics), DNAseI and $30 \mathrm{mM}$ imidazole. After centrifugation the clarified lysate was incubated with Ni-NTA agarose beads (Qiagen) at $4^{\circ} \mathrm{C}$ for 1 hour. Beads were washed five times with phosphate buffer containing DNAseI and $30 \mathrm{mM}$ imidazole. Proteins were eluted in phosphate buffer containing $500 \mathrm{mM}$ imidazole in $1 \mathrm{~mL}$ fractions. The second fraction was aliquoted, flash frozen in liquid nitrogen and stored at $-80^{\circ} \mathrm{C}$. Protein concentration was determined using coomassie staining of the purified protein on an SDS-PAGE gel including a BSA reference and subsequent densitometry of the bands (Figure S2E). Used concentrations refer to Kin4-Ic monomers. 


\section{Preparation of microtubule seeds}

To prepare stabilized seeds, $10 \mu \mathrm{L}$ of MRB80 (80 mM PIPES, pH 6.8, 1 mM EGTA, $4 \mathrm{mM}$ $\mathrm{MgCl}_{2}$ ) containing $0.4 \mu \mathrm{M}$ Alexa 568-tubulin, $0.3 \mu \mathrm{M}$ biotin-tubulin and $15.3 \mu \mathrm{M}$ unlabelled tubulin was polymerised at $37^{\circ} \mathrm{C}$ in the presence of $1 \mathrm{mM}$ GMPCPP (guanosine-5'-[( $\alpha, \beta$ )methyleno]triphosphate) for 30 minutes. Seeds were then pelleted using an airfuge (Beckman Coulter) operated at $\sim 22$ psi for $5 \mathrm{~min}$, resuspended in $90 \mu \mathrm{L}$ of MRB80 and stored at room temperature.

\section{Glass treatment}

Coverslips for microtubule polymerisation assays were first cleaned in acetone for 30 minutes and then with a solution composed of $10 \%(\mathrm{w} / \mathrm{v}) \mathrm{KOH}$ mixed with isopropanol in a 2:1 ratio for 1 hour. It was subsequently silanized using a solution of $4.81 \mathrm{mM}$ dichlorodimethylsilane (DDS) dissolved in trichloroethylene and washed twice in ethanol.

\section{In vitro microtubule growth assays}

Microtubule growth assays were performed in flow chambers consisting of DDS-treated coverglass mounted on a glass slide separated by thin layer of silicone vacuum grease. This generated a small channel with a typical volume of $8 \mu \mathrm{L}$. The channel was incubated with goatanti-biotin (Sigma B3640) dissolved in PBS for 5 min and after being rinsed with PBS, blocked with 1\% Pluronic F-127 in PBS for 1 hour. After flushing the channel with MRB80, GMPCPPstabilized seeds were added. Unbound seeds were removed by flushing the channel with MRB80 supplemented with $80 \mathrm{mM} \mathrm{KCl}$ and $1 \mathrm{mg} / \mathrm{mL}$ casein. Finally, $20 \mu \mathrm{L}$ of assay buffer was introduced, consisting of MRB80 with $80 \mathrm{mM} \mathrm{KCl}, 1 \mathrm{mg} / \mathrm{mL}$ casein, $1 \mathrm{mM}$ ATP, $1 \mathrm{mM}$ GTP, $16.67 \mu \mathrm{g} / \mathrm{mL}$ catalase (Sigma), $33.33 \mathrm{mM}$ D-glucose, $166.7 \mu \mathrm{g} / \mathrm{mL}$ glucose oxidase (Sigma), 0.45 $\mu \mathrm{M}$ Alexa 568-tubulin, $21.2 \mu \mathrm{M}$ unlabelled tubulin (Cytoskeleton inc.) and the indicated amount of Kin4-Ic-(794). The sample was then transferred to a TIRF microscope for observation where it was kept at $30 \pm 1{ }^{\circ} \mathrm{C}$ using an objective heater.

\section{Analysis of microtubule growth velocities}

The growth velocities for a given experimental condition were determined by kymograph analysis. The catastrophe frequencies were determined by dividing the total number of observed catastrophe events by the total time microtubules spend in the growth phase. The error of the catastrophe rate was calculated by dividing the catastrophe rate by the square root of the number of observations (Walker et al. 1988).

\section{Fluorescence microscopy and staining}

All live cell imaging was performed on a Roper spinning disc microscope composed of a Nikon Ti eclipse body, 100x Plan Apo VC objective (NA 1.40), Evolve 512 camera (Photometrics), Yokogawa CSU-X1 spinning disc head with a 1.2x post-magnification lens fitted between the spinning disc unit and the camera. Excitation light for citrine and GFP probes was provided by a $491 \mathrm{~nm}$ Cobolt Calypso50 laser and emitted light was filtered with a 527/60 bandpass filter (Semrock). For FM4-64 and mCherry probes a $561 \mathrm{~nm}$ Cobolt Jive50 laser line was used and emission light was filtered through a 595/50 bandpass filter (Chroma). Typical exposures were 500-700 ms for citrine, GFP and FM4-64 and 800-1000 ms for mCherry.

FM4-64 was dissolved in $\mathrm{dH}_{2} \mathrm{O}$ and used at a final concentration of $10 \mu \mathrm{M}$. The dye was added to dividing cells at the moment of nuclear envelope breakdown, which is roughly $10 \mathrm{~min}$ 
before anaphase onset (Kosetsu et al. 2013). Aniline Blue was used at a final concentration of $0.1 \%(\mathrm{w} / \mathrm{v})$ in $0.1 \mathrm{M} \mathrm{K}_{2} \mathrm{HPO}_{4}$. Tissue fixed in $2 \%(\mathrm{w} / \mathrm{v})$ paraformaldehyde and $0.025 \%(\mathrm{v} / \mathrm{v})$ glutaraldehyde was stained for 20-30 minutes after which it was rinsed twice with $0.1 \mathrm{M} \mathrm{K}_{2} \mathrm{HPO}_{4}$ and mounted in the same solution for observation.

For imaging of the in vitro microtubule growth assay a TIRF setup was used. This consisted of a Nikon Ti eclipse microscope body equipped with a 100x Apo TIRF objective (NA 1.49) and a QuantEM 512SC camera (Photometrics). Excitation light was provided by a Cobolt Jive50 561 $\mathrm{nm}$ laser line and emission light was filtered using a 607/36 bandpass filter. An exposure time of $300 \mathrm{~ms}$ was used to acquire images at $5 \mathrm{~s}$ intervals.

\section{Differential interference contrast (DIC) microscopy}

DIC microscopy was performed on a Nikon Ti eclipse microscope equipped with Normarski optics using a long working distance condenser (NA 0.52) and 100x Plan Apo VC objective (NA 1.40). Illumination was supplied by a CoolLED pE-100 light source. The lid of the glass bottom dish was removed before imaging and the sample was rotated such that the forming cell plate was perpendicular to the prism shear axis. Images were captured using an Andor iXon3 camera. The MAP65a-citrine signal was subsequently recorded using spinning disc microscopy essentially as described above.

\section{Transmission electron microscopy}

For transmission electron microscopy of protonemal cells, small fragments of protonemal tissue grown on BCD agar plates overlaid with cellophane were fixed in $0.1 \mathrm{M}$ sodium phosphate buffer (pH 7.4) containing 2.5\% (v/v) glutaraldehyde, $0.5 \%(\mathrm{w} / \mathrm{v})$ paraformaldehyde and $0.001 \%$ $(\mathrm{v} / \mathrm{v})$ triton $\mathrm{x}-100$. Samples were postfixed in $1 \%(\mathrm{w} / \mathrm{v})$ osmium tetroxide in phosphate buffer, dehydrated in a graded series of ethanol and subsequently embedded in Spurr low-viscosity embedding resin (Sigma EM0300). Ultrathin sections ( $90 \mathrm{~nm}$ ) were made and observed with a JEOL JEM1011 transmission electron microscope operated at an acceleration voltage of $80 \mathrm{kV}$.

\section{Image analysis}

All image analysis was performed using ImageJ and in-house developed Matlab (Mathworks) code, available on request.

The moment of anaphase onset in time lapse movies of mitotic events was determined either using direct observation of chromosome separation or, when chromosomes could not be distinguished, using the rise in MAP65 midplane labelling intensity. The latter method was validated in a strain expressing both GFP labelled MAP65 and a histone-mRFP chromosome marker (data not shown).

To generate average intensity profiles of MAP65a-citrine or SCAMP4-mCherry perpendicular to the forming cell plate, the images were rotated (bicubic interpolation) such that the division plane was oriented vertically with the growing tip of the cell located on the right hand side. The gradual tilting of the division plane common in caulonemal cells, was countered using the ImageJ StackReg plugin (Rigid body type translation). The signal of the division plane was isolated from the remainder of the image using automated thresholding based on the maximum entropy method. The centre of the intensity distribution in each horizontal line of the thresholded area was then calculated using intensity-weighted averaging. The calculated centres were used to align the horizontal lines which were finally averaged along the vertical axis. To generate kymographs 
displaying the evolution of the intensity profiles for MAP65 (Figure 2B), the profiles were normalized per time point to the brightest pixel.

Velocities of radial expansion were obtained by kymograph analysis of StackReg-treated movies (see above). A line following the division plane was used for generating a kymograph of the MAP65a-citrine signal. The slopes of the two shoulders present in the resultant kymograph were then used to calculate velocities for the two leading edges.

The average thickness of the cross walls observed using transmission electron microscopy was calculated by dividing the surface area of the cross wall by the length of the cross wall.

\section{Statistical data analysis}

For statistical validation, data were first tested for normality using the Shapiro-Wilk test $(<50$ samples) or Kolmogorov-Smirnov test ( $\geq 50$ samples). When normality was assumed ( $>0.05)$, data were evaluated using unpaired $t$-tests. For data where a normal distribution was not assumed, Mann-Whitney U and Kruskal-Wallis tests were employed.

\section{Acknowledgements}

The MBL in Woods Hole (MA) is gratefully acknowledged for use of equipment. We thank Aniek Jongerius for help with the in vitro experiments, Ken Kosetsu for sharing MAP65labeled moss lines, and Kyowa Hakko Kogyo for kindly providing driselase. The work has been financially supported by HFSP grant RGP0026/2011 to M.E.J. and G.G. 


\section{Supplemental figures}

A

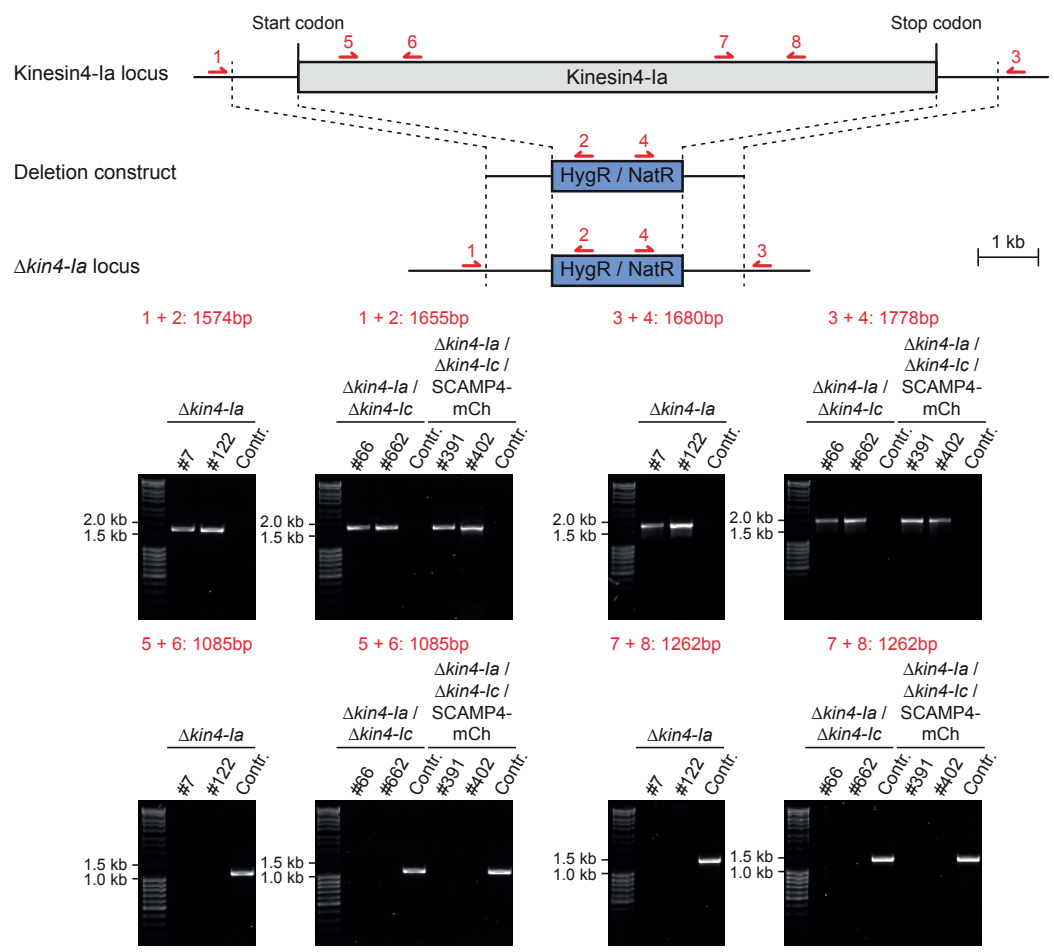

2

B
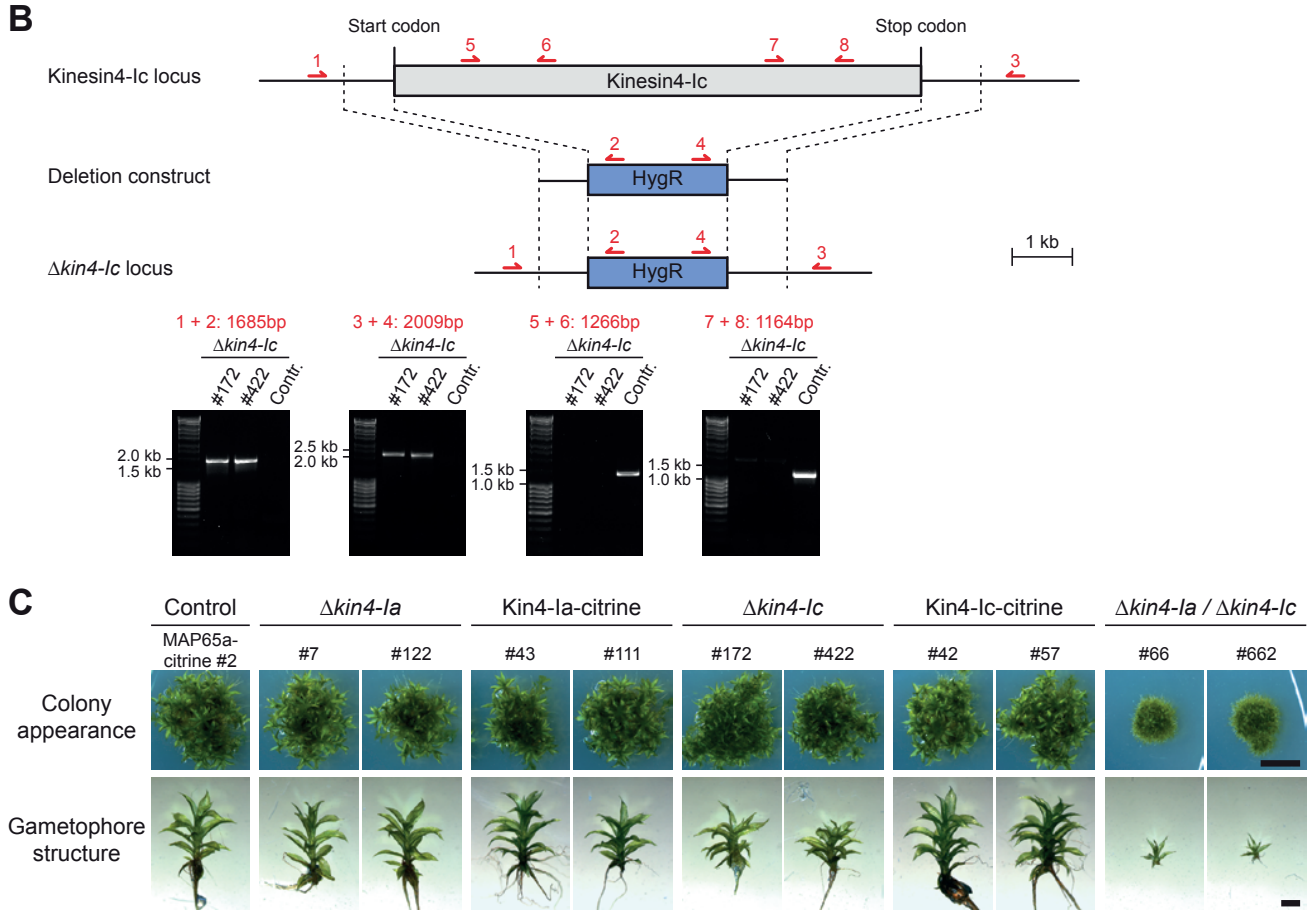

(figure legend on next page) 
Figure S1 - Generation, confirmation and appearance of Kinesin-4 deletion mutants. (A) Schematic representation of the genomic locus containing the Kin4-la encoding gene (gray box). Introduction of the deletion construct by homologous recombination (dashed lines) led to replacement of the gene with a hygromycin or nourseothricin resistance cassette (blue box). Red arrows denote primer binding sites used for confirmation of the obtained lines. The products obtained after PCR reactions using different primer pairs and their predicted sizes are given below. Two independent transformants were tested per line. (B) Schematic of deletion of the Kin4-Ic encoding gene, similar as described in A. (C) Colony and gametophore appearance of moss lines in which Kin4-la and/or Kin4-Ic were absent or labelled with citrine. For each moss line two independent transformants are shown. Kin4-la deletion mutants and Kin4-la citrine-labelled lines exhibited no aberrant colony appearance. Albeit similar in size, colonies of Kin4-Ic deletion lines produced stunted gametophores. No such defects were present in lines expressing Kin4-Ic-citrine, strongly suggesting this is a functional fusion protein. Lines in which both Kinesin-4's were absent showed a reduced colony diameter and gametophores of severely reduced stature. Plants were photographed after 5 weeks of growth on BCD agar plates. The assay was repeated with similar outcomes. The scalebars represent $5 \mathrm{~mm}$ and $1 \mathrm{~mm}$ in the top and bottom rows respectively. 
A

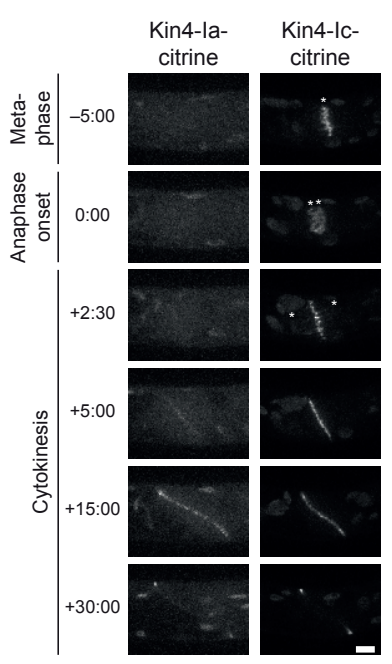

C

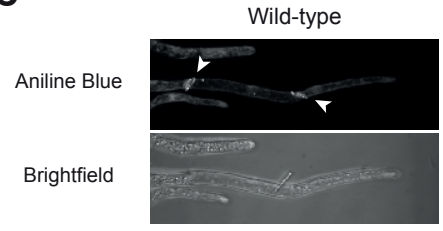

$\Delta k i n 4-/ c$

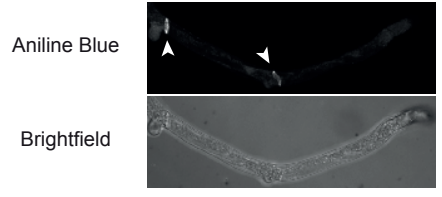

E

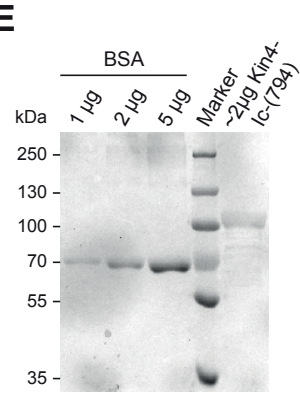

B

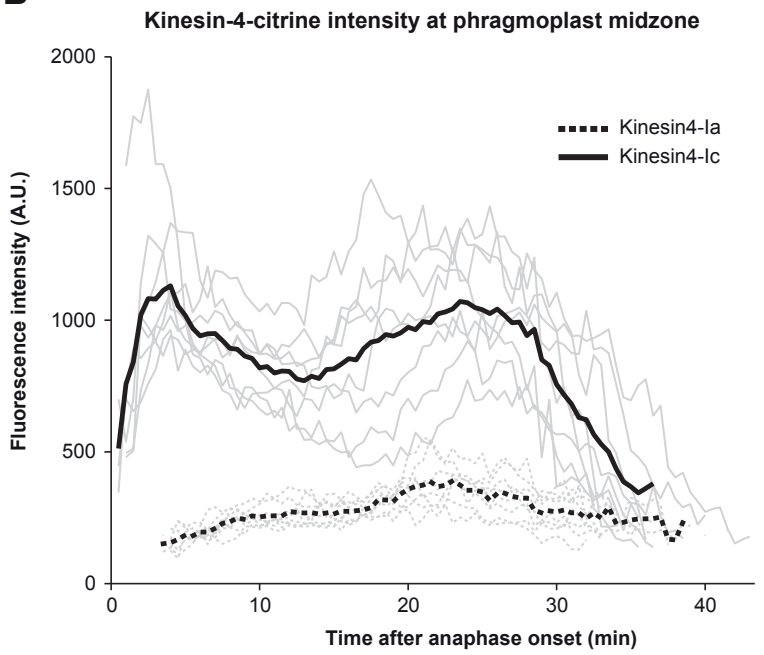

D
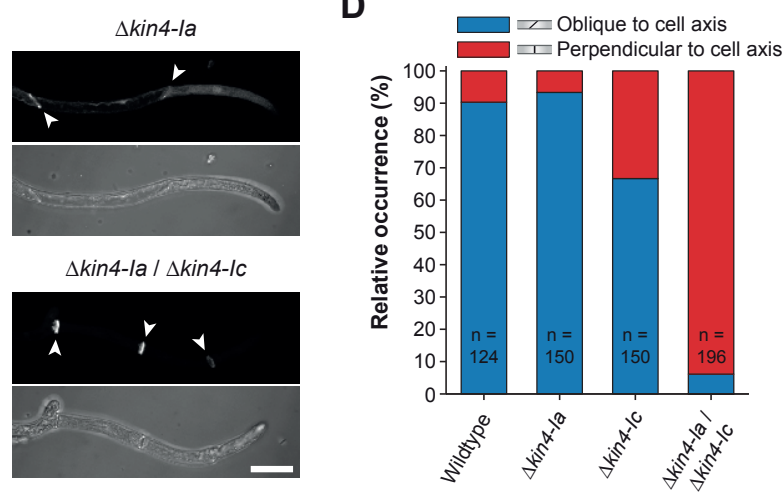

G

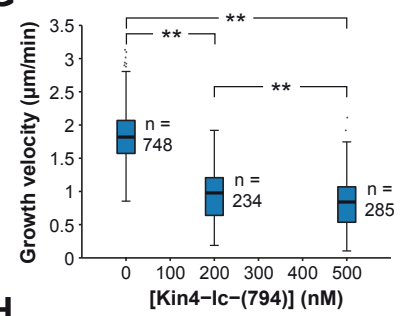

H

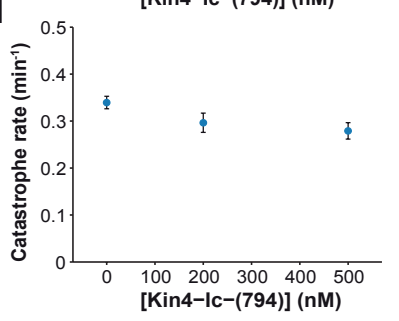

(figure legend on next page) 
Figure S2 - Differential arrival of kinesin-4s at the phragmoplast midplane, division plane rotation defects in absence of kinesin-4s and in vitro microtubule growth inhibition by Kinesin4-Ic. (A) Localization dynamics during cell division in cells with endogenous Kin4-la or Kin4-Ic labelled with citrine. Kin4-Ic localized to chromosomes (marked with an asterix) and rapidly transferred to the phragmoplast midplane at anaphase onset. In contrast, Kin4-la was absent from chromosomes and was gradually recruited onto the midplane from the cytosol about 5 minutes after anaphase onset onwards. Shown images are maximum z-projections of 3 confocal planes spaced $0.5 \mu \mathrm{m}$ apart. Scalebar indicates $5 \mu \mathrm{m}$. (B) Average pixel intensities of citrine-labelled Kin4-la and Kin4-Ic measured within the midplane throughout cytokinesis. Pixels of the midplane were isolated from the remainder of the image using an intensity threshold calculated using the maximum entropy method. The background signal measured in the cytosol was subtracted from the recorded intensities. For both proteins, profiles for 8 cells are shown together with their average value. All analysed cells were imaged with identical illumination and detection settings. (C) Cross wall orientation in kinesin-4 mutants visualized using Aniline Blue staining. The arrowheads mark the callose-rich cross walls. Images are maximum z-projections of 20 planes spaced $1.5 \mu \mathrm{m}$ apart. Scalebar indicates 50 $\mu \mathrm{m}$. (D) Relative occurrence of oblique and perpendicular cross walls in caulonema of kinesin-4 mutants as judged by eye. The indicated number of cross walls were scored in the apical and first two subapical caulonema cells of Aniline Blue stained tissue. The tissue was grown on BCD agar plates overlaid with cellophane. (E) Purified Kin4-IC-(794) protein detected as a single band of approximately the expected size of $89.82 \mathrm{kDa}$ on a coomassie-stained SDS-PAGE gel. Different amounts of BSA were loaded for densitometry. (F) Kymographs depicting microtubule growth initiated from stabilized seeds in presence of the indicated amount of purified Kin4-Ic-(794). Microtubules were visualized using Alexa568-tubulin. The slightly brighter labelled seed section is indicated below with a red bar. The horizontal scalebar represents $5 \mu \mathrm{m}$, the vertical scalebar $1 \mathrm{~min}$. (G) Boxplots of microtubule growth velocity in presence of different amounts of Kin4-lc-(794). The whiskers indicate minimum / maximum observed values or 1.5 times the interquartile range when outliers were present. The indicated number of growth events were analysed for each condition in at least 2 independent experiments. ${ }^{* *}$ indicates differences that were supported with $\mathrm{P}<0.001$ in Mann-Whitney $\mathrm{U}$ and Kruskal-Wallis tests. (H) Catastrophe rates plotted for different amounts of Kin4-Ic-(794). The total time microtubules were observed to spend in growth was at least $700 \mathrm{~min}$ for each condition. Error bars represent standard deviations assuming a Poisson distribution. 
A

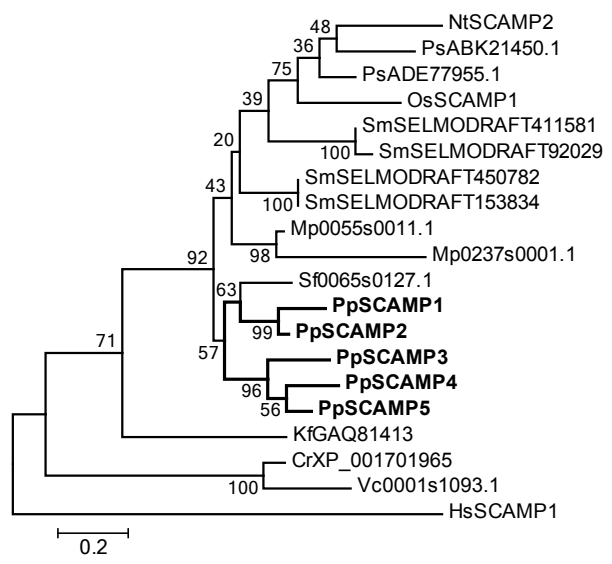

B

\begin{tabular}{clc} 
Name & v1.6 identifier & v3.3 identifier \\
\hline SCAMP1 & Pp1s50_114V6 & Pp3c25_8700V3.1 \\
\hline SCAMP2 & Pp1s297_59V6 & Pp3c5_14240V3.1 \\
\hline SCAMP3 & Pp1s58_245V6 & Pp3c10_9510V3.1 \\
\hline SCAMP4 & Pp1s257_93V6 & Pp3c1_21730V3.1 \\
\hline SCAMP5 & Pp1s30_332V6 & Pp3c2_16160V3.1
\end{tabular}

D

Time after FM4-64 application + washout
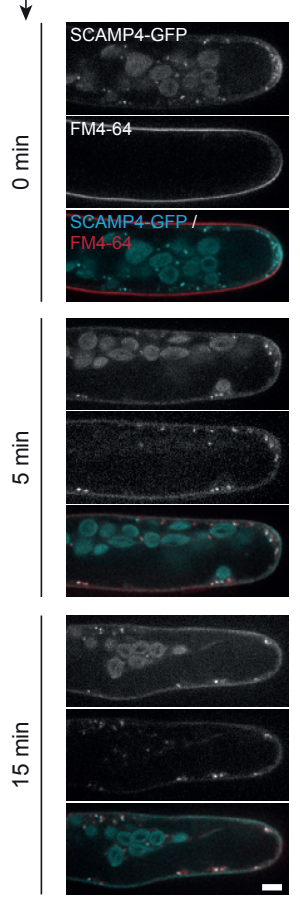

E

SCAMP4-GFP FM4-64

0:00

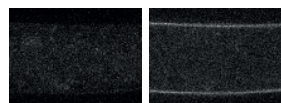

$2: 30$

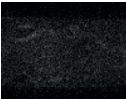

$3: 30$
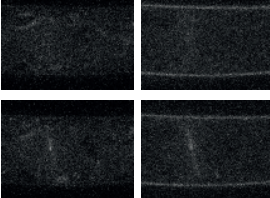

$5: 00$
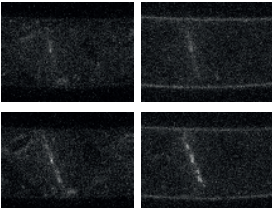

10:00
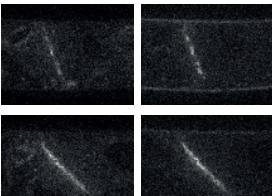

15:00
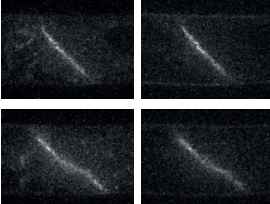

25:00
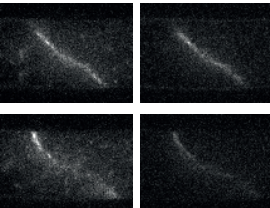

$30: 00$
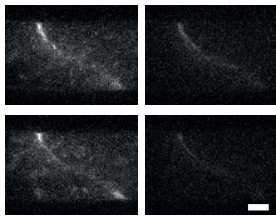

C

SCAMP4 locus

Stop codon
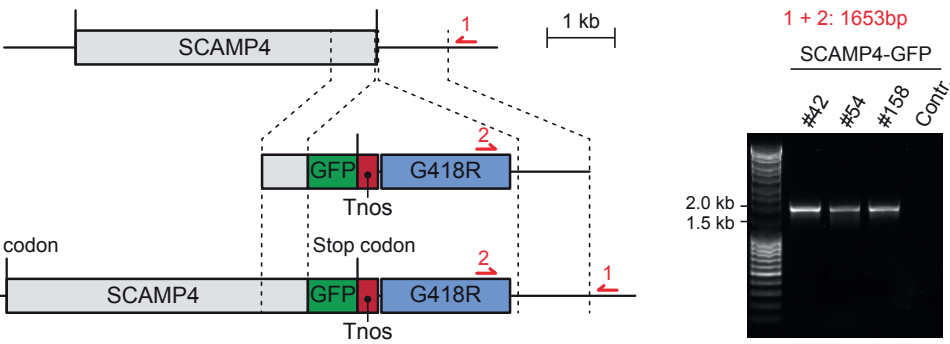

Start codon

Stop codon

SCAMP4 locus
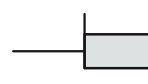

\section{SCAMP4}

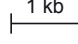

mCherry tagging construct

SCAMP4 mCherry locus

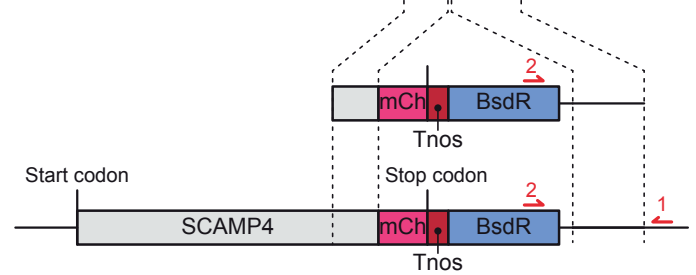

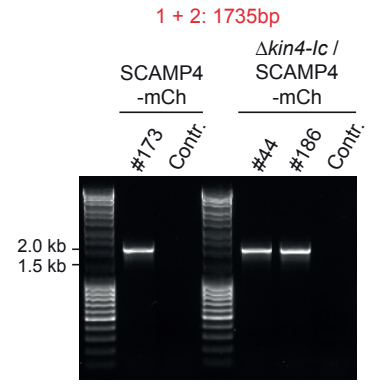

(figure legend on next page) 
Figure S3 - Generation and validation of a labelled moss secretory carrier membrane protein (SCAMP) as cell plate membrane marker. (A) Phylogenetic tree of SCAMPs in non-flowering plants inferred using the maximum likelihood method. The included rice and tobacco SCAMPs have established cell plate localization (Law et al. 2012). Human SCAMP1 served as an outgroup. Bootstrap values (derived from 500 replicates) indicate the percentage of trees in which the associated taxa clustered together. Horizontal branch length represents the number of amino acid substitutions per site. Hs - Homo sapiens, Vc - Volvox carteri, $\mathrm{Cr}$ - Chlamydomonas reinhardtii, $\mathrm{Kf}-\mathrm{Klebsor}$ midium flaccidum, $\mathrm{Pp}$ - Physcomitrella patens, $\mathrm{Sf}$ - Sphagnum fallax, $\mathrm{Mp}$ - Marchantia polymorpha, $\mathrm{Sm}$ - Selaginella moellendorffii, Os - Oryza sativa, Ps - Picea sitchensis, Nt - Nicotiana tabacum. The names used for the Volvox, Sphagnum, and Marchantia proteins are their Phytozome locus identifiers and the names used for Chlamydomonas, Klebsormidium, Selaginella and Picea proteins are their NCBI protein accession numbers. (B) Table listing cosmoss.org locus identifiers for the five SCAMP proteins encoded by the Physcomitrella patens genome using formatting of the indicated version of the genome assembly. (C) Schematic representation of the genomic locus containing the SCAMP4 encoding gene (gray box) and constructs used for C-terminal GFP and mCherry tagging via homologous recombination (dashed lines). In SCAMP4-labelled lines, the original stop codon is replaced with a fragment containing the GFP or mCherry encoding sequence (green and pink boxes respectively), the nopaline synthase terminator (red box) and a cassette conferring G418 or blasticidin resistance (blue box). Red arrows denote primer binding sites used for confirmation of the obtained lines. The product obtained after PCR reaction and its predicted size are given on the right. Despite a number of tested primers and PCR-conditions, integration of the left targeting arm could not be validated. The numbers of independently obtained transformants are given above the gel images. (D) Interphase protonemal cells expressing SCAMP4-GFP treated with FM4-64 in a pulse-chase experiment. Images show three different cells imaged at the indicated time after FM464 application and washout. The colocalization observed after 5 minutes indicated SCAMP4 resides at an early endosomal endomembrane compartment, presumably the trans-Golgi network (Law et al. 2012). A single confocal plane from the cell centre is shown. The scalebar indicates $5 \mu \mathrm{m}$. (E) Dual localization of SCAMP4-GFP and FM4-64 in a dividing caulonemal cell. From the first arrival of vesicles $\sim 3$ minutes after anaphase onset $(t=0)$ onwards, the cell-plate is similarly labelled by both markers. A single confocal plane is shown. The scalebar indicates $5 \mu \mathrm{m}$. 
A

B

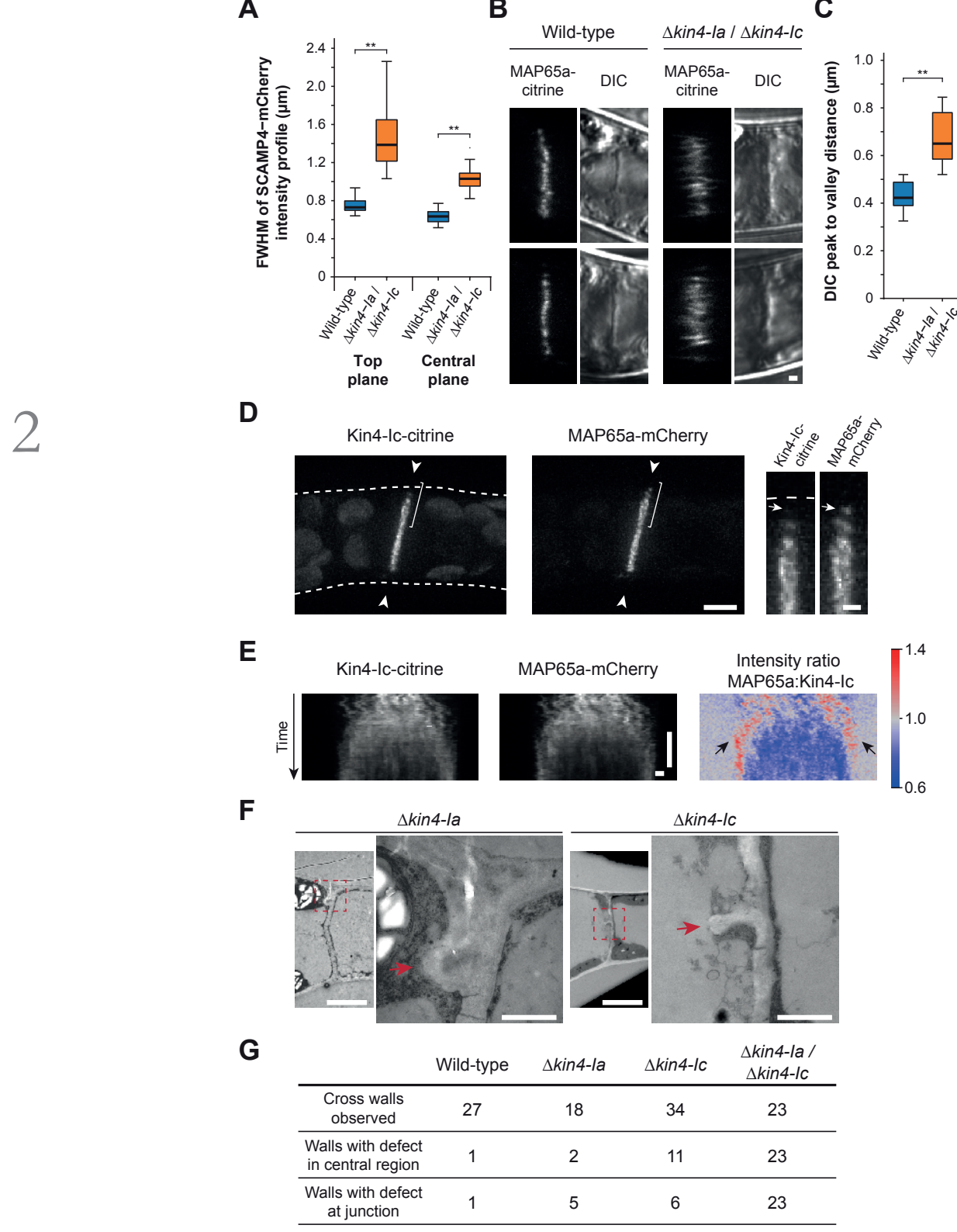

B

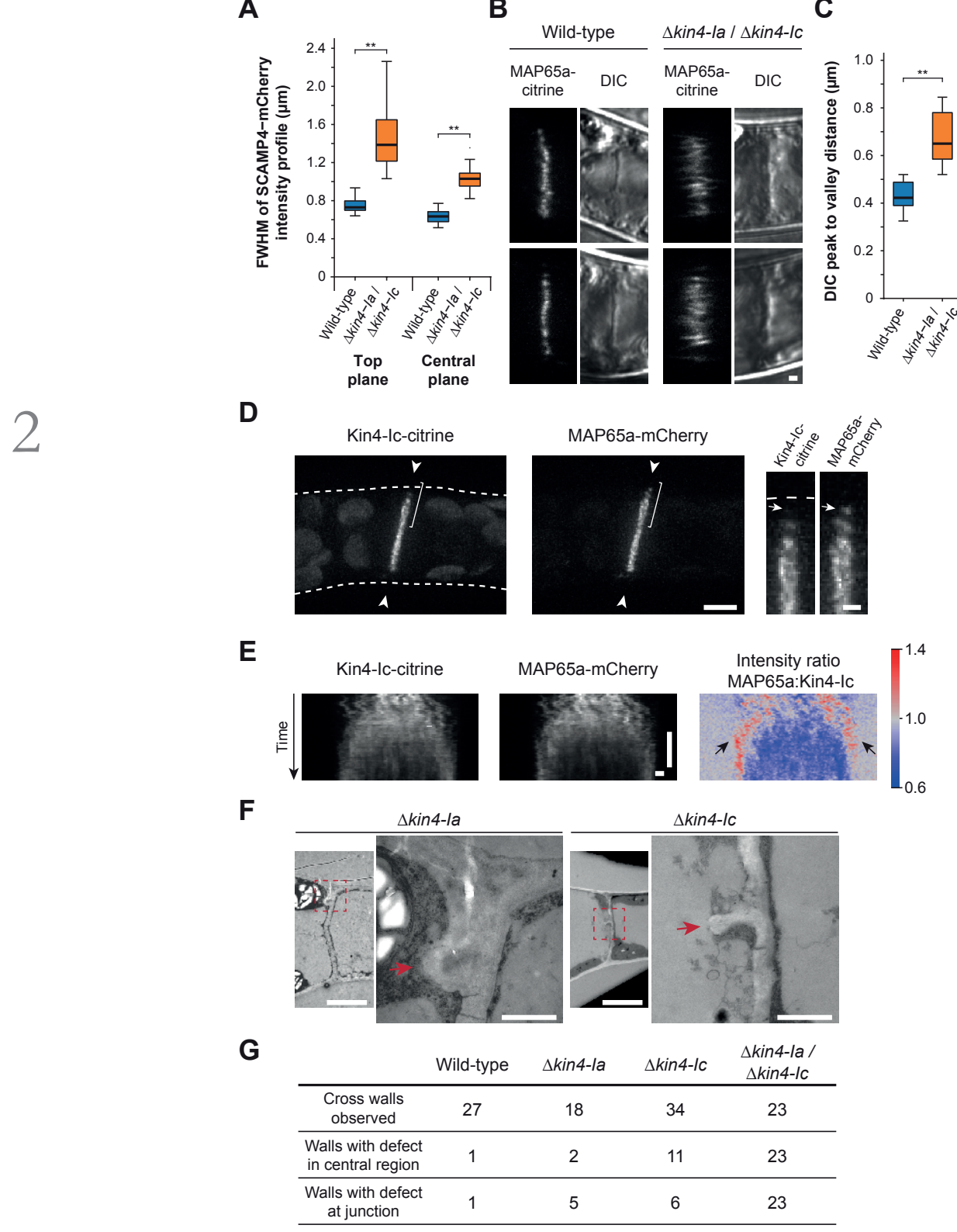

C

Figure S4 - Quantification of cell plate thickening, delayed enrichment of Kinesin-4 at overlaps during phragmoplast expansion and cross wall defects in Kinesin-4 mutants. (A) Boxplots of the full width at half maximum (FWHM) of the SCAMP4-mCherry intensity distribution perpendicular to the cell plate at top and central confocal planes in wild-type $(n=22)$ and $\Delta$ kin4-la / $\Delta$ kin4-Ic $(\mathrm{n}=22)$ cells. The whiskers indicate minimum / maximum observed values or 1.5 times the interquartile range when outliers were present. ${ }^{* *}$ indicates differences that were supported with $\mathrm{P}<0.001$ in Mann-Whitney $U$ and Kruskal-Wallis tests. (B) Cell plates of wild-type and (legend continued on next page) 
kinesin-4 knockout cells observed using differential interference contrast (DIC) microscopy. Corresponding confocal images of the MAP65a-citrine signal are shown on the left. Single optical sections taken at $\sim 3 \mu \mathrm{m}$ from the phragmoplast edge for two cells per genotype are shown. Scalebar indicates $1 \mu \mathrm{m}$. (C) Boxplots of the distance of the bright region on one side of the cell plate (peak) to the dark region on the opposite side (valley) obtained from DIC images of cell plates in wild-type $(n=12)$ and $\Delta k i n 4-l a ~ / \Delta k i n 4-I C$ $(n=15)$ cells. Distances were measured using a linescan of a $1 \mu \mathrm{m}$ wide line segment oriented perpendicular to the cell plate. (D) Still image of a radially expanding phragmoplast ( $4 \mathrm{~min}$ after anaphase onset) with endogenous Kin4-Ic and MAP65a labelled. A magnified view of the bracketed area shows MAP65a-positive overlaps at the expanding edge with little Kin4-Ic present. Arrowheads indicate the line used for generation of the kymographs shown in $\mathrm{E}$. The image is a maximum projection of 5 planes acquired with $0.5 \mu \mathrm{m}$ spacing. Scalebar represents $5 \mu \mathrm{m}$ and $1 \mu \mathrm{m}$ in the enlarged views. (E) Kymographs for Kin4-IC-citrine and MAP65a-mCherry in the phragmoplast midplane indicated in $\mathrm{D}$. The ratio between the two signals is represented on the right. At the two expanding phragmoplast edges (indicated with arrows) a relative deficiency of Kin4-Ic is apparent, while otherwise the Kin4-Ic signal is equal to, or exceeding that of MAP65. Images are representative for 8 visualized cell divisions. Horizontal scalebar indicates $1 \mu \mathrm{m}$ and the vertical scalebar indicates $5 \mathrm{~min}$. (F) Transmission electron micrographs of cross walls in $\Delta k i n 4-l a$ and $\Delta k i n 4-l c$ mutant cells. An enlarged view of the boxed areas is shown on the right. Irregular protrusions from the cross walls are marked with an arrow. Compare with Figure 3G. Scalebars indicate 5 $\mu \mathrm{m}$ in the left images and $1 \mu \mathrm{m}$ in the enlarged views. (G) Table showing quantification of the number of cross walls with a structural defect. A defect was defined as a local protrusion in the cross wall with a length at least 0.5 times the thickness of the cross wall, as exemplified in F. Defects were recorded separately in the centre of the cross wall and at the T-shaped junctions between the cross wall and maternal cell wall that represent the former attachment sites of the cell plate. 


\section{References}

Bieling, P., Telley, I. \& Surrey, T., 2010. A minimal midzone protein module controls formation and length of antiparallel microtubule overlaps. Cell, 142(3), pp.420-432.

Boruc, J. \& van Damme, D., 2015. Endomembrane trafficking overarching cell plate formation. Current Opinion in Plant Biology, 28, pp.92-98.

Bringmann, H. et al., 2004. A kinesin-like motor inhibits microtubule dynamic instability. Science, 303(5663), pp.1519-1522.

Brunkard, J.O., Runkel, A.M. \& Zambryski, P.C., 2015. The cytosol must flow: Intercellular transport through plasmodesmata. Current Opinion in Cell Biology, 35, pp.13-20.

Derivery, E. et al., 2015. Polarized endosome dynamics by spindle asymmetry during asymmetric cell division. Nature, 528(7581), pp.280-285.

Drakakaki, G., 2015. Polysaccharide deposition during cytokinesis: Challenges and future perspectives. Plant Science, 236, pp.177-184.

Duellberg, C. et al., 2013. End-binding proteins and Ase1/PRC1 define local functionality of structurally distinct parts of the microtubule cytoskeleton. Trends in Cell Biology, 23(2), pp.54-63.

Euteneuer, U. \& Mcintosh, J.R., 1980. Polarity of midbody and phragmoplast microtubules. Journal of Cell Biology, 87, pp.509-515.

Fendrych, M. et al., 2010. The Arabidopsis exocyst complex is involved in cytokinesis and cell plate maturation. The Plant Cell, 22(9), pp.3053-3065.

Gaillard, J. et al., 2008. Two microtubule-associated proteins of Arabidopsis MAP65s promote antiparallel microtubule bundling. Molecular Biology of the Cell, 19(10), pp.4534-4544.

Green, R.A., Paluch, E. \& Oegema, K., 2012. Cytokinesis in animal cells. Annual Review of Cell and Developmental Biology, 28, pp.29-58.

Gu, X. \& Verma, D.P., 1996. Phragmoplastin, a dynamin-like protein associated with cell plate formation in plants. The EMBO Journal, 15(4), pp.695-704.

Han, X. \& Kim, J.Y., 2016. Integrating hormone- and micromolecule-mediated signaling with plasmodesmal communication. Molecular Plant, 9(1), pp.46-56.

Hepler, P.K. \& Jackson, W.T., 1968. Microtubules and early stages of cell-plate formation in the endosperm of Haemanthus katherinae Baker. Journal of Cell Biology, 38(2), pp.437-446.

Hiwatashi, Y. et al., 2008. Kinesins are indispensable for interdigitation of phragmoplast microtubules in the moss Physcomitrella patens. The Plant Cell, 20(11), pp.3094-3106.

Hiwatashi, Y., Sato, Y. \& Doonan, J.H., 2014. Kinesins have a dual function in organizing microtubules during both tip growth and cytokinesis in Physcomitrella patens. The Plant Cell, 26(3), pp.1256-1266.

Ho, C.-M.K. et al., 2011. Interaction of antiparallel microtubules in the phragmoplast is mediated by the microtubule-associated protein MAP65-3 in Arabidopsis. The Plant Cell, 23(8), pp.2909-2923.

Hu, C.-K. et al., 2011. KIF4 regulates midzone length during cytokinesis. Current Biology, 21(10), pp.815824.

Janson, M.E. et al., 2007. Crosslinkers and motors organize dynamic microtubules to form stable bipolar arrays in fission yeast. Cell, 128(2), pp.357-368.

de Keijzer, J. et al., 2017. Shortening of microtubule overlap regions defines membrane delivery sites during plant cytokinesis. Current Biology, 27(4), pp.514-520.

de Keijzer, J., Mulder, B.M. \& Janson, M.E., 2014. Microtubule networks for plant cell division. Systems and Synthetic Biology, 8(3), pp.187-194.

Kong, Z. et al., 2015. Kinesin-4 functions in vesicular transport on cortical microtubules and regulates cell wall mechanics during cell elongation in plants. Molecular Plant, 8(7), pp.1011-1023.

Kosetsu, K. et al., 2013. MICROTUBULE-ASSOCIATED PROTEIN65 is essential for maintenance of phragmoplast bipolarity and formation of the cell plate in Physcomitrella patens. The Plant Cell, 25(11), pp.4479-4492.

Kurasawa, Y. et al., 2004. Essential roles of KIF4 and its binding partner PRC1 in organized central spindle midzone formation. The EMBO Journal, 23(16), pp.3237-3248.

Law, A.H.Y., Chow, C.M. \& Jiang, L., 2012. Secretory carrier membrane proteins. Protoplasma, 249(2), pp.269-283.

Lee, K.Y., Davies, T. \& Mishima, M., 2012. Cytokinesis microtubule organisers at a glance. Journal of Cell Science, 125(15), pp.3495-3500. 
Lee, Y.J., Giang, H.M. \& Liu, B., 2001. A novel plant kinesin-related protein specifically associates with the phragmoplast organelles. The Plant Cell, 13(11), pp.2427-2439.

Lekomtsev, S. et al., 2012. Centralspindlin links the mitotic spindle to the plasma membrane during cytokinesis. Nature, 492(7428), pp.276-279.

Miki, T. et al., 2014. Endogenous localizome identifies 43 mitotic kinesins in a plant cell. Proceedings of the National Academy of Sciences of the United States of America, 111(11), pp.1053-1061.

Müller, S. et al., 2004. The plant microtubule-associated protein AtMAP65-3/PLE is essential for cytokinetic phragmoplast function. Current Biology, 14(5), pp.412-417.

Müller, S. \& Jürgens, G., 2015. Plant cytokinesis - No ring, no constriction but centrifugal construction of the partitioning membrane. Seminars in Cell and Developmental Biology, 53, pp.10-18.

Murata, T. et al., 2013. Mechanism of microtubule array expansion in the cytokinetic phragmoplast. Nature Communications, 4, p.1967.

Nakaoka, Y. et al., 2015. Cytoplasmic nucleation and atypical branching nucleation generate endoplasmic microtubules in Physcomitrella patens. The Plant Cell, 27(1), pp.228-242.

Otegui, M.S. et al., 2001. Three-dimensional analysis of syncytial-type cell plates during endosperm cellularization visualized by high resolution electron tomography. The Plant Cell, 13(9), pp.2033-2051.

Otegui, M.S., Verbrugghe, K.J. \& Skop, A.R., 2005. Midbodies and phragmoplasts: analogous structures involved in cytokinesis. Trends in Cell Biology, 15(8), pp.404-413.

Rybak, K. et al., 2014. Plant cytokinesis is orchestrated by the sequential action of the TRAPPII and exocyst tethering complexes. Developmental Cell, 29(5), pp.607-620.

Samuels, A.L., Giddings, T.H. \& Staehelin, L.A., 1995. Cytokinesis in tobacco BY-2 and root tip cells: a new model of cell plate formation in higher plants. Journal of Cell Biology, 130(6), pp.1345-1357.

Schmiedel, G., Reiss, H.D. \& Schnepf, E., 1981. Associations between membranes and microtubules during mitosis and cytokinesis in caulonema tip cells of the moss Funaria bygrometrica. Protoplasma, 108(1-2), pp.173-190.

Staehelin, L.A. \& Hepler, P.K., 1996. Cytokinesis in higher plants. Cell, 84(1), pp.821-824.

Steiner, A. et al., 2016. Cell cycle-regulated PLEIADE/AtMAP65-3 links membrane and microtubule dynamics during plant cytokinesis. The Plant Journal, 88(4), pp.531-541.

Subramanian, R. et al., 2010. Insights into antiparallel microtubule crosslinking by PRC1, a conserved nonmotor microtubule binding protein. Cell, 142(3), pp.433-443.

van der Vaart, B. et al., 2013. CFEOM1-associated kinesin KIF21A is a cortical microtubule growth inhibitor. Developmental Cell, 27(2), pp.145-160.

Walker, R.A. et al., 1988. Dynamic instability of individual microtubules analyzed by video light microscopy: rate constants and transition frequencies. Journal of Cell Biology, 107(4), pp.1437-1448.

Wheatley, S.P. \& Wang, Y.L., 1996. Midzone microtubule bundles are continuously required for cytokinesis in cultured epithelial cells. Journal of Cell Biology, 135(4), pp.981-989.

Yamada, M., Miki, T. \& Goshima, G., 2016. Imaging mitosis in the moss Physcomitrella patens. In P. Chang \& R. Ohi, eds. Methods in Molecular Biology (Clifton, N.J.). New York, NY, United States: Springer New York, pp. 263-282. 


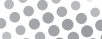

: 8898

:98989

$\because \because \because$

889

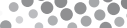

\title{
Chapter 3 - Antiparallel microtubule
}

$\therefore$ Overlaps position exocyst subunit

$\because$ Sec6 during plant cell division

\author{
Jeroen de Keijzer,
}

Han Tang,

Els Sweep,

Marcel E. Janson and

Tijs Ketelaar

Laboratory of Cell Biology, Wageningen:

University, Droevendaalsesteeg 1, $6708 \mathrm{~PB}$

Wageningen, The Netherlands

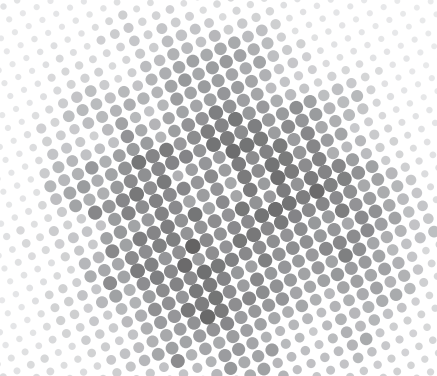

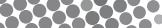

8289888 :

208288 .

\section{$+2$}

.

$898 \%$ :

88:

"You are confined only by the walls you build yourself."

- Andrew Murphy

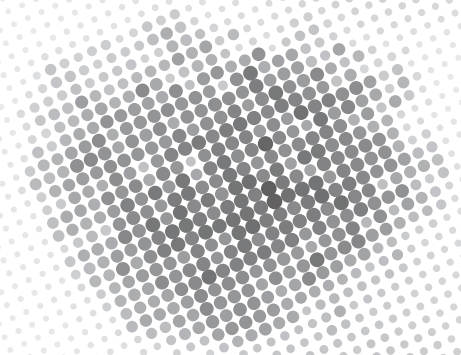




\section{Abstract}

In eukaryotic cells membrane-bound vesicles are trafficked among various intracellular compartments and to and from the plasmamembrane. The fusion of a transport vesicle with its destination membrane is brought about by tethering and fusion complexes. The same machinery is used to build de novo membrane compartments through vesicle-vesicle fusion, such as during plant cytokinesis. Here, vesicles are combined into a transient, radially expanding membrane compartment termed the cell plate. The microtubules of the bipolar phragmoplast apparatus support cell plate biogenesis which ultimately results in the cell wall dividing the daughter cells. In the moss Physcomitrella patens the width of the initial aggregation of cell-plate membrane material is templated by the length of overlap between phragmoplast microtubules. Based on these observations we hypothesize that vesicle tethering and fusion machinery is positioned by the overlap-specified plane. We therefore analysed in moss the subcellular localization of several subunits of the exocyst, a tethering complex active during plant cytokinesis. We find that Sec6, but neither Sec3a, Sec5b nor Sec5d, localizes to microtubule overlap regions prior to cell plate construction and that this localization is partially dependent on the presence and length of these structures. We further demonstrate that moss Sec6 possesses a conserved ability to physically interact with the moss orthologue of the Arabidopsis Sec1/Munc18 protein important for cell-plate membrane fusion called KEULE, and co-localizes with this protein at the division plane. Thus, Sec6 has a high potential to bridge vesicle tethering and fusion activity during plant cytokinesis. The precise role of Sec6-overlap association for its function is unclear at the moment, although the exposed behaviour certainly opens up unique opportunities to learn about the molecular mechanisms involved in exchange of positional cues by the phragmoplast microtubules and the forming cell plate.

\section{Introduction}

In eukaryotic cells the fusion of transport vesicles with endomembrane compartments and the plasma membrane relies on the combined action of fusion and tethering complexes. The force driving the fusion of a vesicle and its destination membrane is almost universally generated by SNARE (soluble N-ethylmaleimide-sensitive factor attachment receptor) complexes, typically composed of 4 conserved, membrane-associated proteins (Söllner et al. 1993; Wickner \& Schekman 2008; Südhof \& Rothman 2009). Tethering - the establishment of the initial physical connection between the two membranes - on the other hand, is orchestrated by a multitude of unrelated, multimeric complexes (Koumandou et al. 2007; Vukašinović \& Žárský 2016). To enable targeted trafficking among the various distinct membrane compartments in eukaryotic cells, their membranes require identity and regulation of what membrane fusion reactions should and should not be compatible. Although SNARE complex composition bestows some of this specificity (Paumet et al. 2004), other factors including Rab GTPases (Grosshans et al. 2006; Stenmark 2009) and the various tethering complexes play an important role as well (Yu \& Hughson 2010). Indeed, each of the several tethering complexes typically facilitates the docking of vesicles at a specific target membrane (Vukašinović \& Žárský 2016; Yu \& Hughson 2010). Although in most studies investigating vesicle tethering the target membrane belongs to a preexisting subcellular compartment, cells sometimes rely on fusing vesicles with each other for the de novo formation of a membrane compartment. A striking example of such a special occasion of vesicle fusion is found in plant cytokinesis. 
The physical separation of the two daughter cells formed during plant cell division occurs via a transient, disk-shaped membrane compartment that expands radially towards the parental cell wall. This membrane compartment is termed the cell plate and its construction culminates in a new cell wall segment dividing two individual plasma membranes (Drakakaki 2015; Müller \& Jürgens 2015). The initiation of the cell plate and its radial expansion relies on the fusion of vesicles supplied mainly by the secretory pathway (Mcmichael \& Bednarek 2013; Richter et al. 2014; Boruc \& van Damme 2015). Since the shape and orientation of the cell plate are reflected in the architecture and position of the final cross wall, timely fusion of vesicles occurring in a confined space constitutes a prime opportunity to position the division plane. Thus, studying the components that drive cell plate membrane fusion and their spatiotemporal regulation could give fundamental insight in both plant cytokinesis and how tethering complexes operate in the context of vesicle-vesicle fusion.

While work in Arabidopsis has uncovered much about the composition, trafficking and function of SNARE complexes fusing cell plate vesicles (reviewed in Müller \& Jürgens 2015; Jürgens et al. 2015), comparatively little is known about the tethering factors involved. In the same model plant both the TRAPPII (Transport Protein Particle II) and exocyst tethering complexes contribute to cell plate biogenesis (Thellmann et al. 2010; Fendrych et al. 2010; Qi et al. 2011; Rybak et al. 2014). Both are multimeric protein complexes that function in post-Golgi membrane trafficking (Drakakaki et al. 2012; Fendrych et al. 2010; reviewed by Vukašinović \& Žárský 2016). Interestingly, while TRAPPII is associated with the cell plate throughout its formation, the exocyst appears briefly on the cell plate during early membrane fusion and then reappears after cell plate insertion (Fendrych et al. 2010; Zhang et al. 2013; Rybak et al. 2014). Nonetheless, the two tethering complexes exhibit physical interaction and most likely function cooperatively during cytokinesis (Rybak et al. 2014). While functional analysis of the TRAPPII complex is expedited by only single genes encoding the two unique TRAPPII subunits (Thellmann et al. 2010; Qi et al. 2011), the diverse complement of genes encoding exocyst subunits have made dissecting the role of the exocyst comparatively cumbersome. Therefore, in this study we set out to investigate exocyst behaviour during cytokinesis in the moss Physcomitrella patens. Although this representative of a basal land plant lineage has seen a similar degree of gene amplification of exocyst subunit genes (with a notable smaller radiation of Exo70 paralogs) (Cvrčková et al. 2012), it features a major haploid phase in its life cycle expediting genetic analysis. Since cell plate assisted cytokinesis is a hallmark of all land plants (Buschmann \& Zachgo 2016) and a comprehensive toolset to study cell division exists for P. patens (Yamada et al. 2016), we anticipate that this model plant is particularly useful for studying exocyst function in relation to cell plate membrane fusion.

In the previous chapter we investigated the microtubules of the cytoskeletal apparatus supporting cell plate formation termed the phragmoplast. We found that antiparallel microtubule overlaps formed in the phragmoplast centre dictate the width of membrane accumulation (de Keijzer et al. 2017). This widening effect was most pronounced during early stages of cell plate assembly, suggesting that the spatial regulation of early membrane transformation processes (i.e. tethering and fusion) strongly depends on overlap dimensions. However, any molecular evidence for such a dependency is currently lacking. To understand if the exocyst could be a cell-plate assembly factor which localization is templated through microtubule overlaps, we monitored the localization of several GFP-tagged exocyst subunits during cytokinesis in the moss P. patens. We show that a single exocyst subunit localizes to microtubule overlaps and that these structures are co-determinants of its localization. This provides the first indication that molecules driving 
membrane fusion processes are at least in part associated with microtubule overlaps during plant cytokinesis. This finding thus makes a case that, in general, spatially confined generation of de novo membrane compartments could be achieved by linking tethering activity to a defined, nonmembranous subcellular structure.

\section{Results and discussion}

The yeast exocyst has been a benchmark in elucidating the structure and subunit assembly of the exocyst complex. Here, the octameric complex is composed of two subcomplexes, each containing 4 subunits that have strong interactions essential for subcomplex integrity (Heider et al. 2015). Limited evidence supports a similar configuration in plants with some additional interactions identified (Figure 1A) (Hála et al. 2008; Zhang et al. 2013). To survey exocyst localization behaviour during cytokinesis in $P$. patens, we focussed on one putative subcomplex and labelled three of its subunits with GFP (Figure 1A). For this purpose a GFP-encoding fragment was integrated at the end of the genes encoding Sec6, Sec3a, and the two Sec5 paralogs Sec5b and Sec5d in a strain expressing $m$ Cherry- $\alpha$-tubulin such that mitotic progression could be monitored (Figure S1). These four subunits were visualized throughout cell division alongside an established cell plate membrane marker expressed by a separate strain for use as temporal reference (SCAMP4; de Keijzer et al. 2017) (Figure 1B). This revealed that Sec3a and Sec5b localized most intensely to the phragmoplast midplane during cell plate initiation and after cell plate attachment to the parental wall, congruent with the localization behaviour of exocyst subunits studied in Arabidopsis (Fendrych et al. 2010; Zhang et al. 2013). Besides these canonical localization dynamics we noted disparate localization behaviour for Sec6 and Sec5d. While Sec5d was absent from the cell plate until late cytokinesis, Sec6 was detected at the centre of the division apparatus from spindle formation onwards, earlier than any other subunit under investigation (Figure 1B, white arrowheads).

Interestingly, all studied subunits also labelled a distinct part of the cell cortex corresponding to the site where the division plane intersected with the plasma membrane (Figure 1B, blue arrowheads). To our knowledge such a cortical localization pattern has not been observed during cytokinesis for any labelled subunit in Arabidopsis (Fendrych et al. 2010; Zhang et al. 2013; Rybak et al. 2014). The cortical labelling was punctate, with numerous puncta together making up a 2-3 $\mu \mathrm{m}$ wide band that formed a continuous ring at the cell cortex (Figure 1C). The dimensions and pattern of this ring match that of the cortical division zone described in higher plants. This zone is a distinct plasma membrane compartment established during preprophase involved in transmitting positional cues to the expanding cell plate for correct division plane establishment (van Damme 2009; Müller et al. 2009; Stöckle et al. 2016). Because the cortical localization of the exocyst could only be detected from early cytokinesis onwards it does not entirely exhibit the temporal distribution of a bona fide cortical division zone protein. However, moss caulonemal cells lack preprophase-bands (Schmiedel et al. 1981), possibly explaining the later arrival. At any rate, it will be interesting to find out if the exocyst has a role in establishment or maintenance of the cortical division zone.

To further characterize the observed differences among the visualized subunits, we identified the moment with respect to anaphase onset at which subunits first became visible at both the phragmoplast midplane and the cortex (Figure 1D-E). This analysis showed that all studied subunits arrived at the cortex at around the same time (5-10 minutes post anaphase onset). 
This could mean that at the cortex the subunits are part of assembled exocyst complexes. The average time of arrival at the midplane however diverged enormously for the studied subunits. While Sec3a and Sec5b were detectable at the phragmoplast midplane at times comparable to the typical appearance time of cell plate membrane ( $\sim 2.5$ minutes after anaphase onset), Sec $5 \mathrm{~d}$ was absent during cell plate formation and expansion and Sec6 was universally present before anaphase had started (Figure 1E). The presence of Sec6 at the site of cell plate biogenesis prior to membrane arrival was verified using co-visualization of Sec6-GFP with the membrane dye FM4-64 (Figure 1F). Thus, while sharing localization at the cortex and late cell plate with other the studied exocyst subunits, Sec6 also exhibits a unique localization to the phragmoplast midplane that is not related to the presence of other studied subunits and even appears to be independent of any membrane compartment.

The observed localization patterns for Sec6 were strongly reminiscent of the sites where microtubules from opposite poles form antiparallel overlaps in the spindle and phragmoplast midplane (Ho et al. 2011; Kosetsu et al. 2013; de Keijzer et al. 2017). To assess if Sec6 localized to regions of microtubule overlap, we generated a moss line expressing Sec6-mCherry together with a citrine-tagged version of the antiparallel microtubule bundling protein MAP65 (Kosetsu et al. 2013). We previously identified a regulator of microtubule dynamics that shortens overlap length at the onset of anaphase: Kin4-Ic (de Keijzer et al. 2017). To see if altered overlap dimension would affect the distribution of Sec6, we similarly tagged MAP65 and Sec6 in a $\Delta$ kin4-Ic genetic background. In wild-type cells, Sec6 localization indeed closely followed the distribution of MAP65-citrine labelled regions of overlap (Figure 2A, left panels). Strikingly, this behaviour was even visible during prophase, when the nuclear envelope is still intact and no bipolar spindle is yet formed (Figure 2A, arrowheads). In absence of the Kin4-Ic regulator of overlap length a similar strong co-localization was observed during prophase and spindle stages, yet Sec6 increasingly localized to only the central region of overlaps as cytokinesis progressed (Figure 2A, right panels). Nevertheless, it was evident that during early cytokinesis the width of the Sec6-mCherry labelled zone was wider in $\Delta$ kint-Ic cells compared to wild-type (Figure 2A, arrows). Thus, taken together these findings suggest that Sec6 localizes to antiparallel overlaps and that during cytokinesis overlap length is an important, but not the absolute determinant, for defining the area of Sec6 localization.

To further understand to what extent microtubule overlaps are a prerequisite for the localization of Sec6 during cytokinesis, we silenced the three MAP65 genes expressed in protonema. Earlier studies have demonstrated that upon knockdown of MAP65 antiparallel microtubule bundle formation in the phragmoplast is severely compromised, but that during early cytokinesis the phragmoplast remains bipolar and still starts aggregating cell-plate membrane in its centre. Later, membrane delivery stalls and newly formed microtubules become less focussed towards the phragmoplast midplane, ultimately resulting in a collapse of the phragmoplast (Kosetsu et al. 2013). Therefore, we deemed the start of cytokinesis useful to study the dependency of Sec6 enrichment at the phragmoplast midplane on the presence of microtubule overlaps formed by MAP65. While in control cells the Sec6-GFP signal increased immediately after the onset of anaphase, in MAP65 silenced cells Sec6-GFP only dimly and diffusely labelled the phragmoplast midplane (Figure 2B, arrowheads). This confirms a role for overlaps in Sec6-localization and prompts future research directed towards elucidating the molecular details of this association.

In conclusion, both disruption of overlap length and the absence of MAP65 affect Sec6 localization dynamics, showing that overlaps are substantial for Sec6 positioning. In addition, 
A

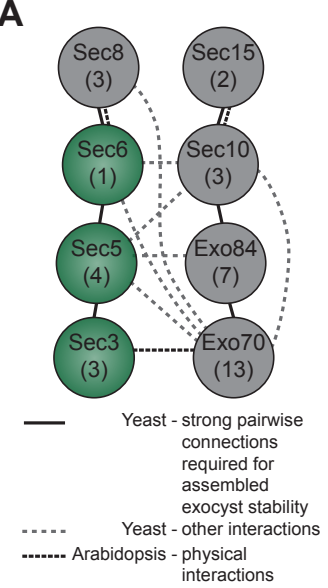

B

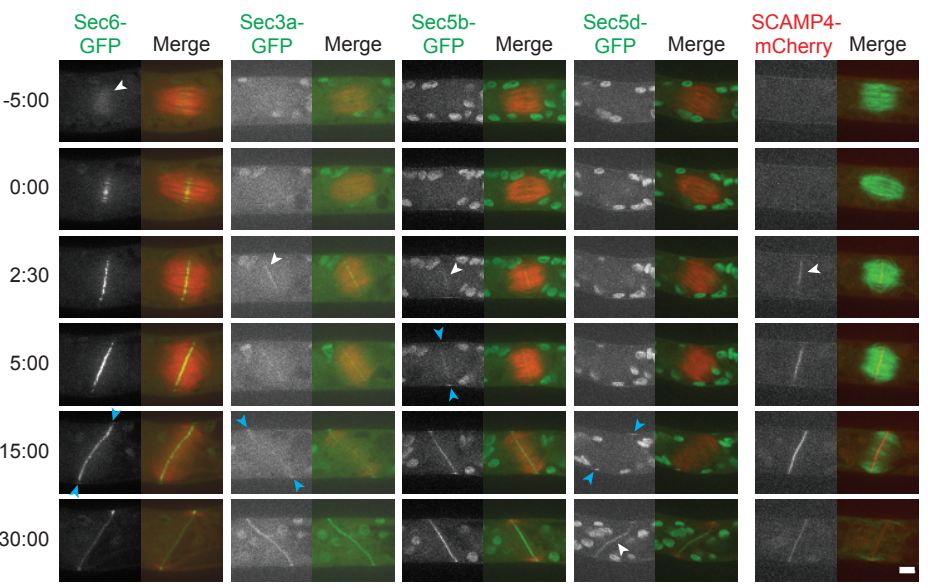

C
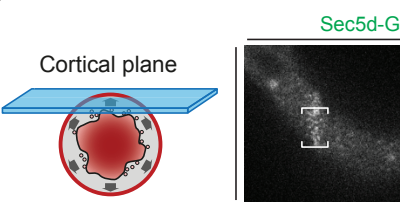

Sec5d-GFP

3
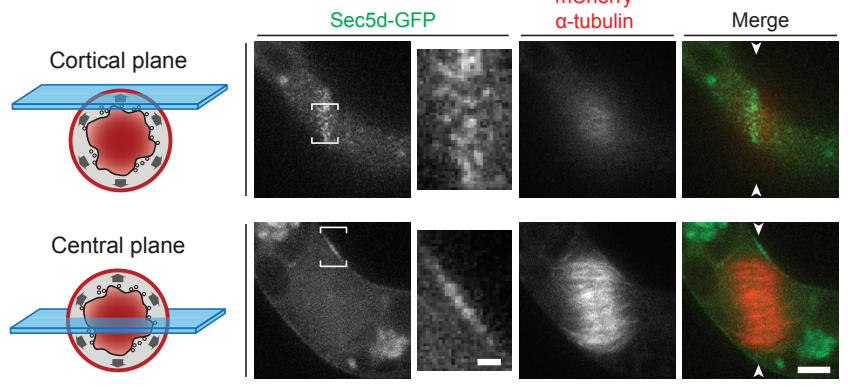

Sideview of division plane

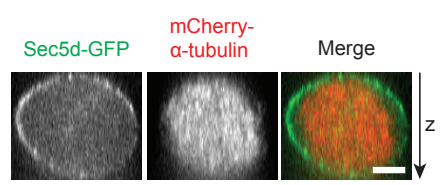

D
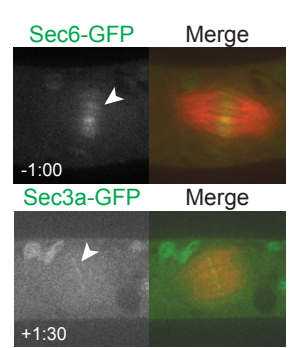

$\mathrm{E}$
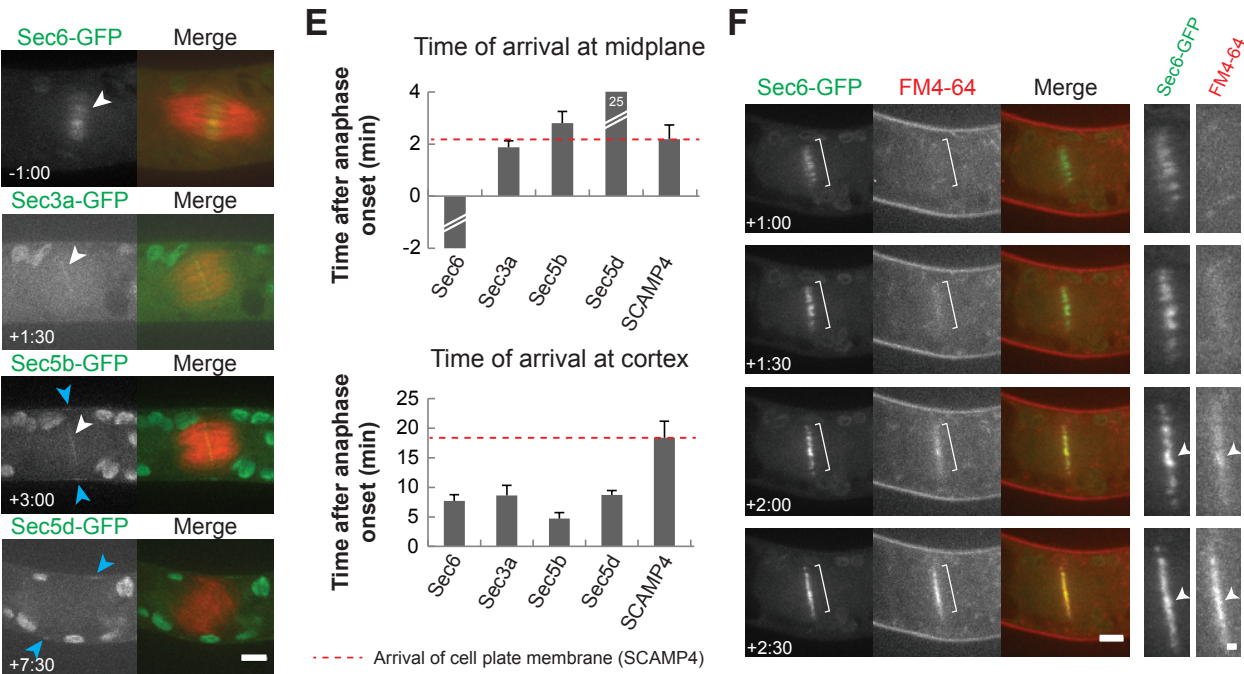

Figure 1 - Localization survey of a subset of Physcomitrella exocyst subunits during cell division. (A) Schematic drawing of the eight canonical exocyst subunits and their physical association. The interactions are mostly derived from the yeast exocyst (Munson \& Novick 2006; Heider et al. 2015), with experimentally confirmed interactions among subunits in plants depicted separately (Hála et al. 2008; Zhang et al. 2013). The numbers between brackets indicate the amount of paralogs encoded in the P. patens genome (Cvrčková et al. 2012). For subunits highlighted in green 
mechanisms independent of MAP65 appear to play a role, especially during later stages of cytokinesis. This hints that Sec6 has affinity for multiple partners during cell division which could have several underlying causes. Firstly, the arrival of other exocyst subunits, which appears related to initial membrane deposition (Figure 1B-D), could sequester Sec6 from overlaps as it is assembled into exocyst complexes. Additionally (or additively), Sec6 could interact with secondary factors, since in yeast the Sec6 subunit interacts with at least three other proteins besides exocyst complex members (Sivaram et al. 2006; de Craene et al. 2006; Morgera et al. 2012). Thus, elucidation of Sec6-interactions active during plant cytokinesis may help uncover the mechanisms in which the enigmatic localization pattern of Sec6 is rooted.

A screen for Sec6-interacting proteins in Arabidopsis revealed that Sec1/munc18 (SM) family protein KEULE physically associated with Sec6, but not with several other cell plate localized exocyst subunits (Wu et al. 2013). Interestingly, KEULE is required for efficient membrane fusion during cytokinesis in Arabidopsis (Assaad et al. 1996). It contributes to fusion via preventing the important cytokinetic SNARE-component KNOLLE from refolding into its closed, nonfusion-competent conformation, thereby allowing the formation of fusogenic trans-SNARE complexes among cell plate delivered vesicles (Park et al. 2012; Karnahl et al. 2017; Jürgens et al. 2015). If and how the interaction with exocyst subunit Sec6 contributes to KEULE function is presently unknown. Nonetheless, in Sec6-mutants cytokinesis defects are found that resemble these encountered in plants lacking KEULE (Wu et al. 2013), hinting that a cooperative Sec6KEULE interaction might be present during cytokinesis. Our observation that Physcomitrella Sec6 exhibits characteristics of both overlap and cell plate-association may thus be related to a similar interaction with a cytokinetic SM family protein. However, this family has not yet been explored in moss.

We identified 7 loci in the P. patens genome that are predicted to encode SM family proteins.

GFP fusions of at least one paralog were created. (B) Localization of exocyst subunits Sec6, Sec3a, Sec5b and Sec5d during cell division visualized in caulonemal apical cells expressing mCherry-a-tubulin (appearing red in the merged image) and a GFP-tagged version of the indicated exocyst subunit. The progression of cell plate and phragmoplast development, visualized with cell plate marker SCAMP4 (de Keijzer et al. 2017) and GFP-a-tubulin, is depicted on the right as a reference. Time after anaphase onset $(\mathrm{t}=0)$ is indicated in min:sec. Localization to the phragmoplast midplane is indicated with white arrowheads, while blue arrowheads point to examples of cortical localization. Images are maximum z-projections of 3 confocal planes spaced $0.5 \mu \mathrm{m}$ apart. Scalebar, $5 \mu \mathrm{m}$. (C) Cortical localization of the exocyst during cell division visualized in a cell expressing Sec5d-GFP and $\mathrm{mCherry}$ - $\alpha$-tubulin at a single cortical and central confocal plane (illustrated on the left). A detailed view of the Sec5d-GFP signal indicated by the brackets is shown. A sideview of the division plane (marked with arrowheads) is depicted on the right. Scalebar in overview and sideview images, $5 \mu \mathrm{m}$; Scalebar in zoomed images, $1 \mu \mathrm{m}$. (D) Frames illustrating the first detectable appearance of exocyst subunits Sec6, Sec3a, Sec5b and Sec5d at the phragmoplast midplane (white arrowheads) and the cell cortex (blue arrowheads). The co-visualized mCherry-a-tubulin signal appears red in the merged image. Images are maximum z-projections of 3 confocal planes spaced $0.5 \mu \mathrm{m}$ apart. Scalebar, $5 \mu \mathrm{m}$. (E) Bar graph showing the average appearance time of GFP-tagged exocyst subunits at the phragmoplast midplane and the cell cortex compared to that of cell plate membrane (SCAMP4-mCherry, dashed line). Error bars indicate standard deviation. Averages were obtained from $n=4$ cells (Sec6 and Sec3a), $n=5$ cells (Sec5b and Sec5d) or $n=8$ cells (SCAMP4). (F) Early cell plate membrane accumulation visualized in a dividing cell expressing Sec6-GFP stained with FM464 membrane dye. Time with respect to anaphase onset $(t=0)$ is indicated in min:sec. The bracketed areas are shown in detail on the right. Here, arrowheads mark membranous material accumulating at Sec6-labelled sites. Images are maximum z-projections of 3 confocal planes spaced $0.5 \mu \mathrm{m}$ apart. Scalebar in overview images, $5 \mu \mathrm{m}$; Scalebar in zoomed images, $1 \mu \mathrm{m}$. 
A

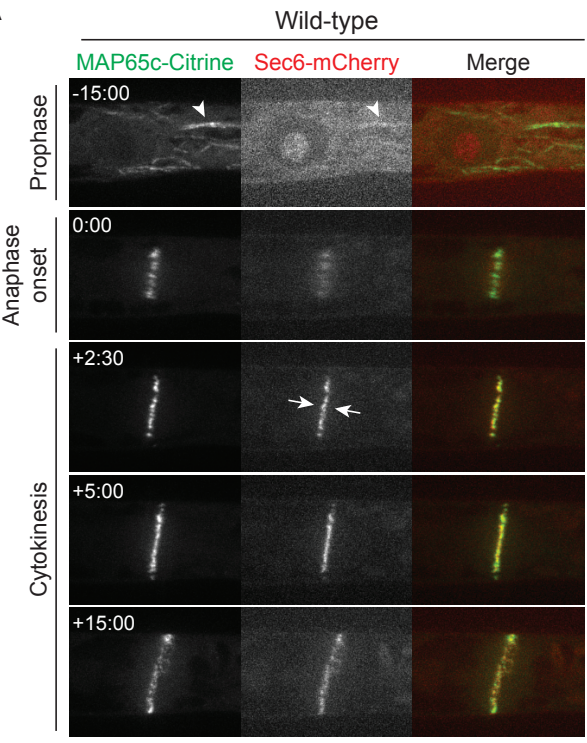

B

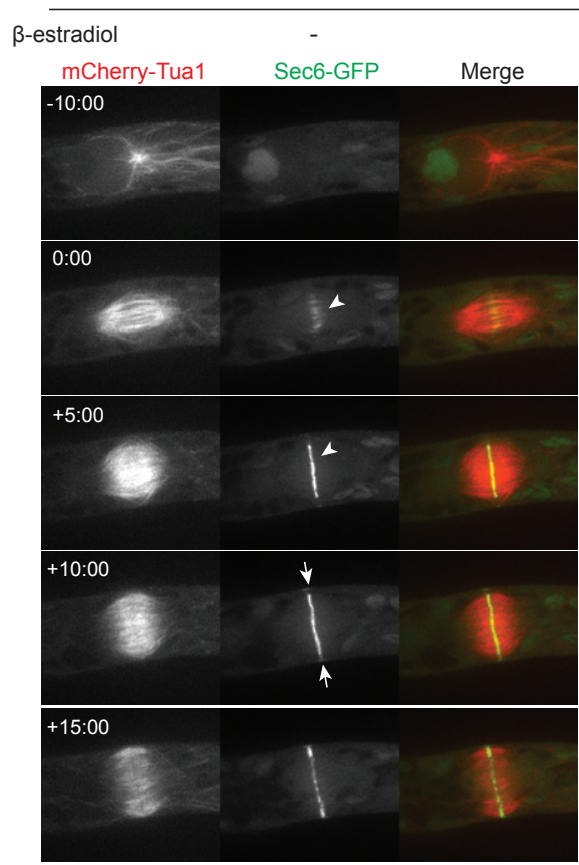

$\Delta k$ in $4-/ c$

MAP65a-Citrine Sec6-mCherry Merge
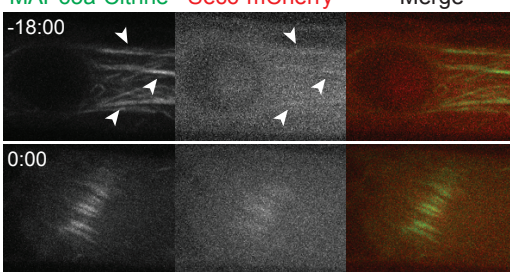

\section{$+2: 30$}

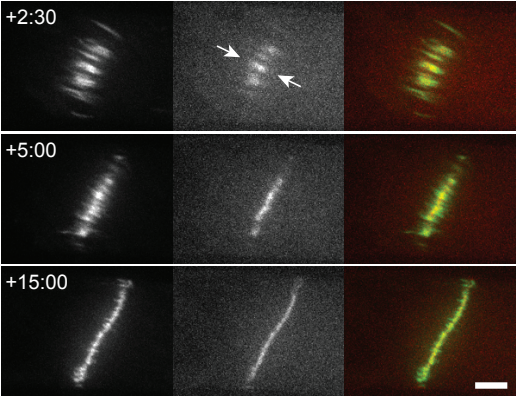

MAP65a-c RNAi

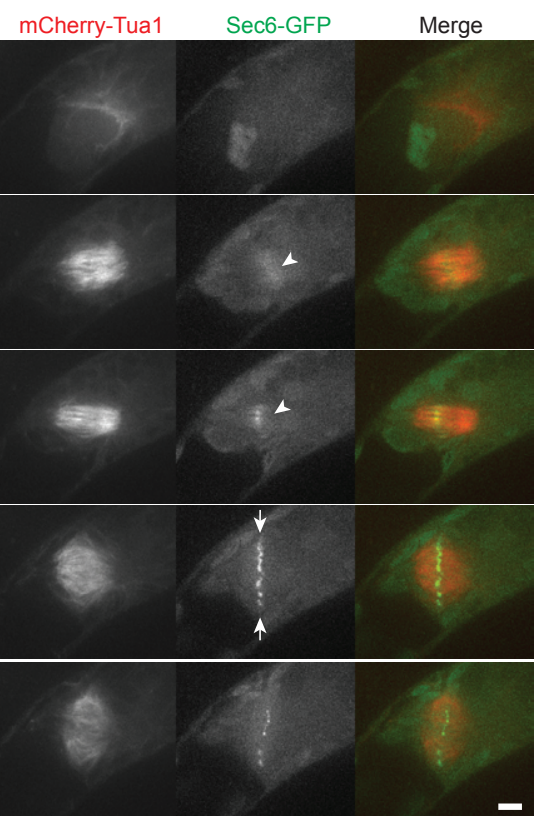

Figure 2 - Localization of exocyst subunit Sec6 to antiparallel microtubule overlaps depends on overlap length and presence of MAP65. (A) Dividing caulonemal cells expressing citrine labelled MAP65 and Sec6-mCherry in a wild-type and $\Delta$ kin4-Ic genetic background imaged throughout mitosis. MAP65-labelled microtubule bundles present during prophase that show concomitant Sec6 labelling are marked with arrowheads. Arrows highlight the width of the Sec6-labelled zone during early cytokinesis. Time with respect to anaphase onset $(t=0)$ is indicated in min:sec. Images are maximum z-projections of 3 confocal planes spaced $0.5 \mu \mathrm{m}$ 
Of the identified loci, two contained genes with a predicted exon number equal to that of the Arabidopsis KEULE gene and their expected gene products exhibited an overall similarity to Arabidopsis KEULE at least twice higher than the other predicted SM genes (Figure 3A). Therefore we considered these two genes encoding potential Arabidopsis KEULE homologues, warranting their further examination. We analysed their gene expression in various tissues using a publically available transcriptome atlas (Ortiz-Ramírez et al. 2016), which revealed that the gene encoded at locus Pp3c17_24130 was ubiquitously expressed in all moss tissues, while the other gene (Pp3c16_570) was only expressed in rhizoids (Figure 3A and Figure S2). Since we used protonemal cells throughout this study we focussed on the former gene and tentatively named it PpKEULE.

Since the position of introns in genes contains information on the evolutionary trajectory of the gene family they belong to (e.g. Rogozin et al. 2003; Garcia-España et al. 2009; Javelle et al. 2011) and the exon number between Arabidopsis KEULE and PpKEULE matched, we compared their intron-exon structure. This revealed that intron positions were highly similar for both species (Figure 3B). Introns in the PpKEULE gene were slightly longer, however this is in line with average intron length being bigger in moss compared to Arabidopsis (Rensing et al. 2005). The concurrent intron-exon structure of the genes of both species signifies that they are derived from a gene present in the last common ancestor of mosses and higher plants, providing a basis for possible conservation of a role in cell-plate membrane fusion.

We next determined whether the identified moss KEULE orthologue interacted with Sec6, as has been described in Arabidopsis by Wu et al. (2013). During their characterization of the physical association between KEULE and Sec6 the interaction domain of KEULE was narrowed to a C-terminal portion of the protein which these authors designated C1 (Wu et al. 2013). The high degree of sequence conservation allowed us to delineate the same domain in PpKEULE and also test it for its ability to interact with Sec6. In a reciprocal yeast 2-hybrid assay with Physcomitrella Sec6, KEULE and the KEULE-C1 domain, we found an interaction between Sec6 and PpKEULE when Sec6 was fused to the activation domain of the Gal4 transcription factor. A strong interaction between the PpKEULE-C1 fragment and Sec6 highlighted that this domain was sufficient for interaction (Figure 3C). When Sec6 was fused to the DNA-binding domain of Gal4 only a weak interaction with the PpKEULE-C1 domain was found (Figure 3C). This was also the case for the Arabidopsis proteins in a similar 2-hybrid reporter assay (Wu et al. 2013), and is possibly because in this case the binding partners are in a less favourable configuration for reconstituting Gal4 function. The PpKEULE protein thus interacts with Sec6 in a fashion indistinguishable from its orthologue in Arabidopsis. This hints that Sec6 with a KEULE-type SM protein could form a conserved module and furthers that Physcomitrella KEULE and Sec6 could play an important role in cell plate membrane fusion.

To establish if the interaction between Sec6 and PpKEULE could function during cell plate formation we visualized Sec6-mCherry together with endogenous PpKEULE tagged with GFP (Figure S1). We chose to fuse GFP to the c-terminal end, since this yielded a functional fusion protein for Arabidopsis KEULE (Steiner et al. 2016). While absent from Sec6-labelled regions

apart. Scalebar, $5 \mu \mathrm{m}$. (B) Time sequence of dividing caulonemal cells expressing Sec6-GFP and mCherry- $\alpha$-tubulin with silencing of MAP65a, b and c uninduced (control) and induced though the addition of $\beta$-estradiol. Arrowheads mark the accumulation of Sec6 to the phragmoplast midplane during early cytokinesis and the arrows delineate the midplane during a later stage of cytokinesis. Time with respect to anaphase onset $(t=0)$ is indicated in min:sec. Images are maximum z-projections of 3 confocal planes spaced $0.5 \mu \mathrm{m}$ apart. Scalebar, $5 \mu \mathrm{m}$. 
during prophase and metaphase, PpKEULE-GFP became rapidly enriched at the site of Sec6mCherry at the onset of cytokinesis (Figure 3D). During radial expansion of the phragmoplast, newly formed sites of Sec6 localization showed relatively little corresponding PpKEULE-GFP signal (Figure 3D, arrowheads). Furthermore, at the cortical zone marked by the labelled exocyst

A

\begin{tabular}{llccc}
$\begin{array}{l}\text { v1.6 locus } \\
\text { identifier }\end{array}$ & $\begin{array}{l}\text { v3.1 locus } \\
\text { identifier }\end{array}$ & $\begin{array}{c}\text { \% identity with } \\
\text { AtKEULE }\end{array}$ & \# of exons & $\begin{array}{c}\text { expressed in } \\
\text { protonema }\end{array}$ \\
\hline Pp1s468_8 & Pp3c17_24130 & $54.50 \%$ & 21 & + \\
Pp1s144_47 & Pp3c16_570 & $49.53 \%$ & 21 & - \\
Pp1s33_94 & Pp3c18_21360 & $23.60 \%$ & 13 & + \\
Pp1s391_41 & Pp3c12_11250 & $21.04 \%$ & 4 & - \\
Pp1s188_85 & Pp3c4_5220 & $20.77 \%$ & 4 & + \\
Pp1s26_105 & Pp3c17_5740 & $18.09 \%$ & 22 & + \\
Pp1s203_96 & Pp3c9_21860 & $16.44 \%$ & 10 & +
\end{tabular}

B

AtKEULE

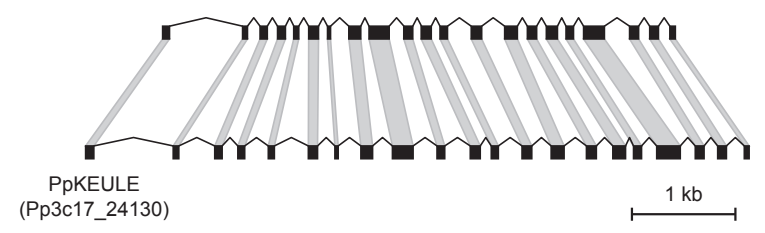

D

Sec6-mCherry PpKEULE-GFP Merge

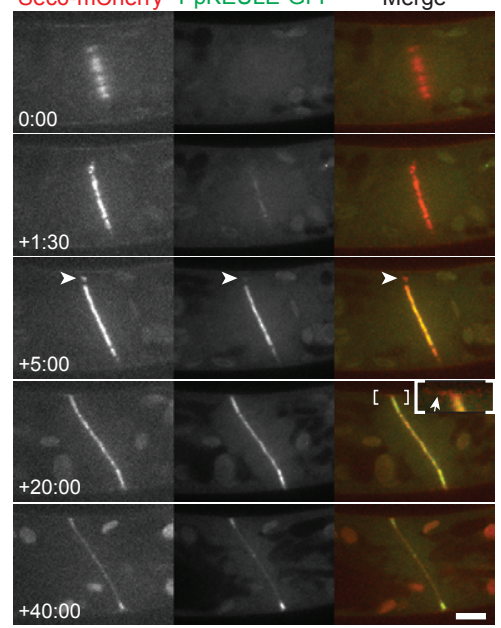

C

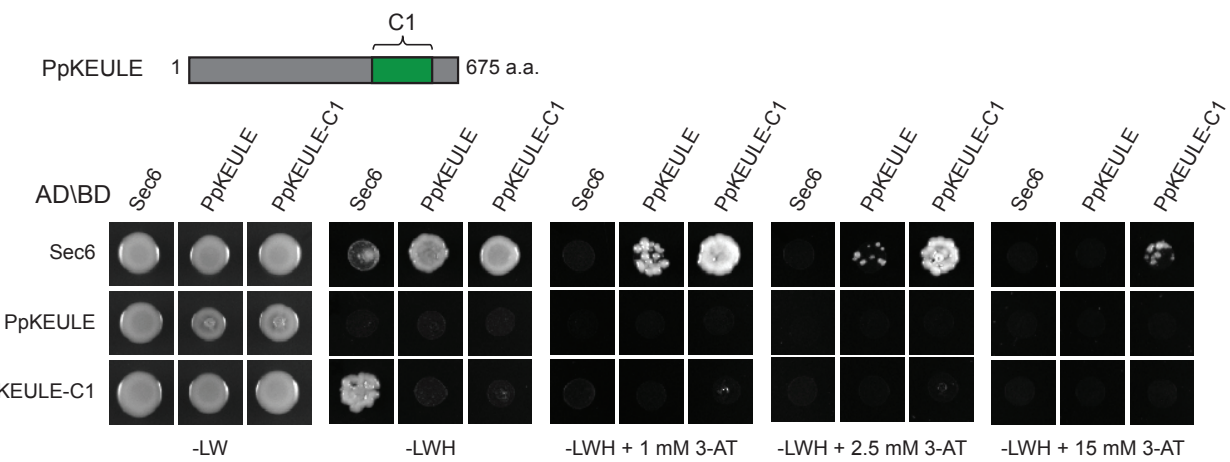

Figure 3 - Identification of a $P$. patens KEULE orthologue that physically interacts with Sec6 and co-localizes at the phragmoplast midplane during cell plate formation. (A) Table summarizing loci putatively encoding Sec1/munc18-like (SM) family proteins and their identity to the cytokinetic Sec1 protein KEULE of Arabidopsis at the protein level. The locus identifiers for two versions of the $P$. patens genome assembly are given. For each predicted gene the number of exons and the presence of indicators of gene expression in protonemal tissue are given. (B) Comparison between the exon (black boxes) and intron (connecting chevrons) structure of the gene encoding Arabidopsis KEULE and the putative P. patens orthologue expressed in protonema. (C) Yeast-twohybrid interaction assay between Sec6, PpKEULE, and the PpKEULE C1 domain (illustrated at the top). Proteins were fused with the Gal4 activation domain (AD) and binding domain (BD) and their different combinations were co-expressed in yeast strains that were tested for growth on reporter media lacking histidine in combination with increasing amounts of the competitive inhibitor 3-AT. (D) Sec6 and PpKEULE co-visualized throughout cell division in a cell expressing Sec6-mCherry and PpKEULE-GFP. The arrowheads indicate Sec6-labelled regions at the expanding edge of the phragmoplast showing less associated KEULE-GFP compared to the central regions. An enlarged view of the bracketed area is depicted in the inset where the arrow points to Sec6 located at the cortex without associated PpKEULE-GFP signal. Images are maximum z-projections of 3 confocal planes spaced $0.5 \mu \mathrm{m}$ apart. Scalebar, $5 \mu \mathrm{m}$. 
subunit no corresponding PpKEULE signal was found (Figure 3D, arrow). Taken together, this spatially and temporally imperfect co-localization might mean that physical association between Sec6 and PpKEULE in a cytokinetic context is tightly regulated. Functional analysis of these two factors may provide further insight in this.

From recent advances in the field of vesicle fusion a picture is emerging wherein tethering complexes rather universally exert control over SNARE action (Hong \& Lev 2014). For the exocyst complex a lot of this crosstalk seems to impinge on the Sec6 subunit. In yeast Sec6 binds to SNARE component Sec9, preventing it from being incorporated into a SNARE complex (Sivaram et al. 2005; Morgera et al. 2012) or otherwise affecting Sec9 function (Dubuke et al. 2015). Furthermore, it physically interacts with one of the founders of the SM family proteins, Sec1, to modify SNARE activity (Hashizume et al. 2009; Morgera et al. 2012). The Sec6-Sec1 interaction can occur in the context of an assembled exocyst complex, indicative of a direct regulatory link between tethering and fusion activities (Morgera et al. 2012; Heider et al. 2015). Our results on Physcomitrella Sec6 localization during plant cytokinesis and interaction with a conserved SM protein suggest a similar bridging role. During cell plate biogenesis, Sec6 could link tethering activity with activation or modulation of the vesicle fusion machinery, currently most obviously via the SM protein KEULE. Very unique about Sec6 during plant cytokinesis however, is its localization to microtubule overlaps, implying that it also establishes interactions with other subcellular compartments independent of the exocyst or any other fusion machinery component. Of course, for other exocyst subunits for which precedent of Sec6 interaction exists (i.e. Sec8, Sec10 and Exo70; Figure 1A), occurrence of similar localization behaviour should be verified to investigate if Sec6 at microtubule overlaps is not part of a small exocyst subcomplex. From here, the molecular details of the association could be further elucidated. However, most crucially, whether the unique localization of Sec6 to microtubule overlaps is relevant for its putative functions in cell plate vesicle tethering and fusion then remains to be investigated. The requirement of short microtubule overlaps for efficient cell plate formation certainly proposes that this will offer a highly interesting opportunity to further explore how phragmoplast microtubules and the forming cell plate interact.

\section{Materials and methods}

\section{Plasmids and cloning procedures}

For construction of GFP/mCherry tagging constructs for Sec6, Sec3a, Sec5b, Sec5d, and PpKEULE, regions of approximately $1 \mathrm{~kb}$ before and after the stop codon of the encoding gene were amplified by PCR using primers listed in table 1. The PCR fragments were digested with restriction enzymes indicated in table 1 and ligated into correspondingly digested peGFPNPTII or pmCherry-LoxP-BsdR vector (de Keijzer et al. 2017).

To generate the expression constructs used for yeast-two-hybrid assays, first the coding sequences of Sec6, PpKEULE, and the PpKEULE-C1 domain were PCR-amplified from a $P$. patens cDNA library derived from protonemal tissue. The PCR products were then introduced into the pENTR-D-TOPO vector and subsequently subcloned into destination plasmids pDEST22 and pDEST32 (Invitrogen) via Gateway LR reactions. All plasmids used throughout this study are listed in table 2 . 
Table 1 - Primers used in this study

\begin{tabular}{|c|c|c|c|}
\hline Primer & Use & Sequence $\left(5^{\prime}>3^{\prime}\right)$ & RE site \\
\hline HT23 & Cloning of Sec6 left targeting arm & CGGGGTACCCCGACTTCTTACCCAGGTTTG & Kpnl \\
\hline HT24 & Cloning of Sec6 left targeting arm & ACCGTCGAGCTTCCTTCCTGGCCTGTATTTTGC & Xhol \\
\hline HT3 & Cloning of Sec6 right targeting arm & AAAACTAGTCGAGAGAAGGAAATAGTGTTATGAC & Spel \\
\hline HT4 & Cloning of Sec6 right targeting arm & AAAGCGGCCGCGATGACTACAGAATCCCGATTAGC & Notl \\
\hline HT11 & Cloning of Sec3a left targeting arm & ACCGCTCGAGGTGCTGAAGTCAAATTTGCTGATTG & Xhol \\
\hline HT12 & Cloning of Sec3a left targeting arm & $\begin{array}{l}\text { AAAGATATCGAAAATGAAGTTCTTGAAGATTTCT- } \\
\text { TTCATCTC }\end{array}$ & EcoRV \\
\hline HT13 & Cloning of Sec3a right targeting arm & ACGGGATCCGGGGTAGGTTGTAGAATGATGGTC & BamHI \\
\hline HT14 & Cloning of Sec3a right targeting arm & $\begin{array}{l}\text { AGAAGCGGCCGCGGTTGACTTAATACAAAGAGTCTA- } \\
\text { CATCCC }\end{array}$ & Notl \\
\hline EO9 & Cloning of Sec5b left targeting arm & TCTGGTACCGGCCATCAAATCTCTCGTGGAAGC & Kpnl \\
\hline EO10 & Cloning of Sec5b left targeting arm & TGCGGGCCCTCTCAAGTCATCGTTATCTACATAGGCG & Apal \\
\hline EO11 & Cloning of Sec5b right targeting arm & TCGACCGGTTTTAGTGCGACGCCGTGCG & Agel \\
\hline EO12 & Cloning of Sec5b right targeting arm & TCCCGAGCGGCCGCCACAAGGCATGTGGGCCAAGC & Notl \\
\hline EO1 & Cloning of Sec5d left targeting arm & TCAGGTACCTGCCGATTGTGTGGTTGCTTTG & Kpnl \\
\hline EO2 & Cloning of Sec5d left targeting arm & $\begin{array}{l}\text { CGCCGTATCGATTCTCAACTCATCGTTATCTACATAAAT- } \\
\text { TTGAGGG }\end{array}$ & Clal \\
\hline EO3 & Cloning of Sec5d right targeting arm & TCTACCGGTCAAGAATTGAAGGCCTCACAGGG & Agel \\
\hline EO4 & Cloning of Sec5d right targeting arm & GGACCCGGGCCGGTGCATCCGACTTTGCTG & Smal \\
\hline JK277 & $\begin{array}{l}\text { Cloning of PpKEULE left targeting } \\
\text { arm }\end{array}$ & ACGCGGGCCCTGTAGGTTGCAGTTACGATGTGG & Apal \\
\hline JK278 & $\begin{array}{l}\text { Cloning of PpKEULE left targeting } \\
\text { arm }\end{array}$ & ACGCGTCGACAAAGTCGTCCATCTCTAATTTGCC & Sall \\
\hline JK279 & $\begin{array}{l}\text { Cloning of PpKEULE right targeting } \\
\text { arm }\end{array}$ & CGGGATCCGAAGGAGCACGAAGGAATTTTTGG & BamHI \\
\hline JK280 & $\begin{array}{l}\text { Cloning of PpKEULE right targeting } \\
\text { arm }\end{array}$ & CGCGAGCTCGTAAATTGACACTCGGGCAAGTGC & Sacl \\
\hline HT122 & $\begin{array}{l}\text { Cloning of Sec6 coding sequence } \\
\text { for } \mathrm{Y} 2 \mathrm{H}\end{array}$ & CACCATGATGATGCATGATGCTGGC & - \\
\hline HT123 & $\begin{array}{l}\text { Cloning of Sec6 coding sequence } \\
\text { for } Y 2 \mathrm{H}\end{array}$ & TCACTTCCTTCCTGGCCTG & - \\
\hline HT118 & $\begin{array}{l}\text { Cloning of PpKEULE coding se- } \\
\text { quence for } Y 2 \mathrm{H}\end{array}$ & CACCATGGATTCCTCGCACGGTTC & - \\
\hline HT119 & $\begin{array}{l}\text { Cloning of PpKEULE coding se- } \\
\text { quence for } \mathrm{Y} 2 \mathrm{H}\end{array}$ & TCAAAAGTCGTCCATCTCTAATT & - \\
\hline HT120 & $\begin{array}{l}\text { Cloning of PpKEULE-C1 coding } \\
\text { sequence for } \mathrm{Y} 2 \mathrm{H}\end{array}$ & CACCCAGCTAGCGAAGTTGAGTGAT & - \\
\hline HT121 & $\begin{array}{l}\text { Cloning of PpKEULE-C1 coding } \\
\text { sequence for } \mathrm{Y} 2 \mathrm{H}\end{array}$ & TCAATCACTGACGGCATGCCTC & - \\
\hline HT57 & $\begin{array}{l}\text { Sec6 GFP/mCherry tagging geno- } \\
\text { typing }\end{array}$ & GCAGGTATGTGGAAGAGAGA & - \\
\hline HT86 & $\begin{array}{l}\text { Sec6 GFP/mCherry tagging geno- } \\
\text { typing }\end{array}$ & CTCTTTACGAGAGGCGACAA & - \\
\hline HT69 & Sec3a GFP tagging genotyping & GGCTCGCTACTATCAACAAGCTAG & - \\
\hline HT70 & Sec3a GFP tagging genotyping & СССАТTAСТСАТTGAАATTCAАATCAAAAACTG & - \\
\hline HT63 & Sec5b GFP tagging genotyping & CACTCATGCGGAGGTTAGCA & - \\
\hline HT64 & Sec5b GFP tagging genotyping & CTCAGTGGTTGGTCCTTGTT & - \\
\hline HT67 & Sec5d GFP tagging genotyping & TGTGCGTCTTTTGGCGGATT & - \\
\hline HT68 & Sec5d-GFP tagging genotyping & GAATGCTATGTGCAAGCGGA & - \\
\hline JK164 & MAP65c citrine tagging genotyping & АCTCCTTGACCGCTTGCTTCC & - \\
\hline
\end{tabular}


Table 1 - Primers used in this study

\begin{tabular}{|c|c|c|c|}
\hline Primer & Use & Sequence $\left(5^{\prime}>3^{\prime}\right)$ & RE site \\
\hline JK202 & MAP65c citrine tagging genotyping & CGTCCAAACGCTGGCTACAAGG & - \\
\hline HT163 & PpKEULE GFP tagging genotyping & CACGATCAGGCTAAACGAC & - \\
\hline HT164 & KEULE GFP tagging genotyping & АСTTTTTTCTCATGCGTAC & - \\
\hline JK219 & $\begin{array}{l}\text { SCAMP4 mCherry tagging geno- } \\
\text { typing }\end{array}$ & TAGCGTTCTGAGGTTCGGGTGG & - \\
\hline JK220 & $\begin{array}{l}\text { SCAMP4 mCherry tagging geno- } \\
\text { typing }\end{array}$ & GTGATCAACGTGGGATTAAAGAAGTCAC & - \\
\hline JK129 & General reverse primer on mCherry & CCCTTGGTCACCTTCAGCTTGG & - \\
\hline JK112 & General reverse primer on GFP & GCCGGACACGCTGAACTTGTG & - \\
\hline JK114 & $\begin{array}{l}\text { General forward primer from resist- } \\
\text { ance cassette }\end{array}$ & ACTCAAGAGGATAAAACCTCACC & - \\
\hline
\end{tabular}

Table 2 - Plasmids used in this study

\begin{tabular}{lll} 
Plasmid & Usage & Source \\
\hline SEC6-GFP & C-terminal GFP tagging of SEC6 & This study \\
\hline SEC3a-GFP & C-terminal GFP tagging of SEC3a & This study \\
\hline PpKEULE-GFP & C-terminal GFP tagging of PpKEULE & This study \\
\hline SEC5b-GFP & C-terminal GFP tagging of SEC5b & This study \\
\hline SEC5d-GFP & C-terminal GFP tagging of SEC5d & This study \\
\hline SEC6-mCherry & C-terminal mCherry tagging of SEC6 & This study \\
\hline SCAMP4-mCherry & C-terminal mCherry tagging of SCAMP4 & (de Keijzer et al. 2017) \\
\hline MAP65c-Citrine & C-terminal citrine tagging of MAP65c & (Kosetsu et al. 2013) \\
\hline MAP65a-c RNAi & B-estradiol induced silencing of MAP65a-c & (Kosetsu et al. 2013) \\
\hline pDEST22-SEC6 & Yeast-2-Hybrid & This study \\
\hline pDEST22-PpKEULE & Yeast-2-Hybrid & This study \\
\hline pDEST22-PpKEULE-C1 & Yeast-2-Hybrid & This study \\
\hline pDEST32-SEC6 & Yeast-2-Hybrid & This study \\
\hline pDEST32-PpKEULE & Yeast-2-Hybrid & This study \\
\hline pDEST32-PpKEULE-C1 & Yeast-2-Hybrid & This study \\
\hline
\end{tabular}

\section{$P$. patens growth conditions and transformation}

P. patens tissues were routinely grown on BCDAT plates under continuous light. Plasmids were linearized and introduced into the $P$. patens genome by homologous recombination using PEG-mediated protoplast transformation (Nishiyama et al. 2000). Correct insertion events were characterised by PCR (Figure S1). Characteristics of generated moss lines and their use throughout the study are summarized in table 3. For imaging, protonemal tissue was grown for 5 to 7 days on BCD medium in glass bottom dishes (Yamada et al. 2016). 
Table 3 - Summary of $P$. patens strains and their usage throughout this study

\begin{tabular}{|c|c|c|c|c|c|c|}
\hline Name & Experimental use & $\begin{array}{l}\text { Main } \\
\text { clone }\end{array}$ & $\begin{array}{l}\text { Background } \\
\text { strain }\end{array}$ & $\begin{array}{l}\text { Plasmid } \\
\text { transformed }\end{array}$ & Figure & Reference \\
\hline EF1-mCherry-tua1 & $\begin{array}{l}\text { Background strain } \\
\text { for tagging exocyst } \\
\text { subunits with GFP }\end{array}$ & \#52 & Wild-type & $\begin{array}{l}\text { EF1-mCherry- } \\
\text { tua1-BsdR }\end{array}$ & - & $\begin{array}{l}\text { (K. Kosetsu, T. } \\
\text { Miki, G. Goshi- } \\
\text { ma, unpublished) }\end{array}$ \\
\hline $\begin{array}{l}\text { Sec6-GFP / mCher- } \\
\text { ry-tua1 }\end{array}$ & $\begin{array}{l}\text { Sec6 visualization } \\
\text { during cell division }\end{array}$ & \#92 & $\begin{array}{l}\text { EF1-mCher- } \\
\text { ry-tua1 }\end{array}$ & Sec6-GFP & $1 \mathrm{~B}, \mathrm{D}, \mathrm{E}$ & This study \\
\hline $\begin{array}{l}\text { Sec3a-GFP / } \\
\text { mCherry-tua1 }\end{array}$ & $\begin{array}{l}\text { Sec3a visualization } \\
\text { during cell division }\end{array}$ & \#57 & $\begin{array}{l}\text { EF1-mCher- } \\
\text { ry-tua1 }\end{array}$ & Sec3a-GFP & $1 B, D, E$ & This study \\
\hline $\begin{array}{l}\text { Sec5b-GFP / } \\
\text { mCherry-tua1 }\end{array}$ & $\begin{array}{l}\text { Sec5b visualization } \\
\text { during cell division }\end{array}$ & \#2 & $\begin{array}{l}\text { EF1-mCher- } \\
\text { ry-tua1 }\end{array}$ & Sec5b-GFP & $1 \mathrm{~B}, \mathrm{D}, \mathrm{E}$ & This study \\
\hline $\begin{array}{l}\text { Sec5d-GFP / } \\
\text { mCherry-tua1 }\end{array}$ & $\begin{array}{l}\text { Sec5d visualization } \\
\text { during cell division }\end{array}$ & \#48 & $\begin{array}{l}\text { EF1-mCher- } \\
\text { ry-tua1 }\end{array}$ & Sec5d-GFP & $1 \mathrm{~B}-\mathrm{E}$ & This study \\
\hline Sec6-GFP & $\begin{array}{l}\text { Sec6 / FM4-64 co-lo- } \\
\text { calization }\end{array}$ & \#37 & Wild-type & Sec6-GFP & $1 \mathrm{~F}$ & This study \\
\hline $\begin{array}{l}\text { GFP-tua1 / } \\
\text { SCAMP4-mCherry }\end{array}$ & $\begin{array}{l}\text { Division apparatus / } \\
\text { cell plate membrane } \\
\text { visualization }\end{array}$ & \#2 & $\begin{array}{l}\text { GFP-a-tubu- } \\
\text { lin1 (GTU14) }\end{array}$ & $\begin{array}{l}\text { SCAMP4-mCher- } \\
\text { ry }\end{array}$ & $1 \mathrm{~B}$ & $\begin{array}{l}\text { This study; } \\
\text { (Hiwatashi et al. } \\
\text { 2008) }\end{array}$ \\
\hline $\begin{array}{l}\text { Sec6-mCherry / } \\
\text { MAP65c-citrine }\end{array}$ & $\begin{array}{l}\text { Sec6 / microtubule } \\
\text { overlap co-locali- } \\
\text { zation }\end{array}$ & $\# 1$ & $\begin{array}{l}\text { Sec6-mCher- } \\
\text { ry }\end{array}$ & MAP65c-citrine & $2 \mathrm{~A}$ & This study \\
\hline $\begin{array}{l}\text { Sec6-mCher- } \\
\text { ry / } \triangle k i n 4-I c / \\
\text { MAP65a-citrine }\end{array}$ & $\begin{array}{l}\text { Sec6 / microtubule } \\
\text { overlap co-locali- } \\
\text { zation upon Kin4-Ic } \\
\text { deletion }\end{array}$ & \#223 & 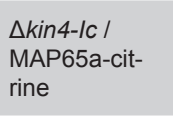 & Sec6-mCherry & $2 A$ & $\begin{array}{l}\text { This study; (de } \\
\text { Keijzer et al. } \\
\text { 2017) }\end{array}$ \\
\hline $\begin{array}{l}\text { MAP65a-c RNAi / } \\
\text { Sec6-GFP / mCher- } \\
\text { ry-tua1 }\end{array}$ & $\begin{array}{l}\text { Sec6 localization } \\
\text { upon microtubule } \\
\text { overlap disruption }\end{array}$ & \#15 & $\begin{array}{l}\text { Sec6-GFP / } \\
\text { mCherry-tua1 }\end{array}$ & MAP65a-c RNAi & $2 B$ & This study \\
\hline $\begin{array}{l}\text { Sec6-mCherry/KE- } \\
\text { ULE-GFP }\end{array}$ & $\begin{array}{l}\text { Sec6 / PpKEULE } \\
\text { co-localization }\end{array}$ & \#2 & $\begin{array}{l}\text { Sec6-mCher- } \\
\text { ry }\end{array}$ & PpKEULE-GFP & $3 \mathrm{D}$ & This study \\
\hline
\end{tabular}

\section{Identification of Physcomitrella Sec1 homologues}

Proteins of the Sec1/Munc18 family were identified by BLAST using the Arabidopsis KEULE protein sequence as input against the predicted proteins of $P$. patens genome assemblies version 1.6 and 3.1 and by keyword search in the Phytozome 10.3 browser (www.phytozome. org). Expression in protonema was assessed by verifying the presence of ESTs derived from protonemal tissue and using the Physcomitrella eFP browser (Ortiz-Ramírez et al. 2016).

\section{Yeast two-hybrid assay}

Yeast 2-hybrid assays were performed with a split Gal4 transcription factor system using the His3 gene as reporter (James et al. 1996). For this, pDEST22/32-based constructs (Table 1) were transformed into yeast strain PJ69-4a or PJ69-4 $\alpha$ by PEG-mediated transformation. Positive transformants with minimal background reporter activity were selected on double dropout medium (-Leu-His or-Trp-His) with different concentrations of 3-amino-1,2,4-triazole (3-AT) present to increase histidine-dependent growth stringency. Selected clones were then allowed to mate and resulting diploids were selected on -Leu-Trp plates. With surviving cells a yeast 2-hybrid assay was then performed on triple dropout medium (-Leu-Trp-His) with increasing amounts of 3-AT present.

\section{Fluorescence microscopy and staining}

All live cell imaging was performed on a Roper spinning disk microscope system composed of a Nikon Ti eclipse body, Yokogawa CSU-X1 spinning disc head and Photometrics Evolve 512 camera. All imaging was conducted with a 100x Plan Apo VC oil immersion objective (NA 1.40), 
using a 1.2x post-magnification fitted before the camera. The GFP and citrine probes were exited using $491 \mathrm{~nm}$ light generated by a Cobolt Calypso50 laser and their emitted light was bandpass filtered at 497-557 nm. For FM4-64 and mCherry $561 \mathrm{~nm}$ excitation light generated by a Cobolt Jive50 laser was used in combination with bandpass filtering at 570-620 nm. During image digitization a camera electron multiplication gain of 300 was employed and typical exposures were 800-1000 ms for GFP, citrine, FM4-64 probes and 1000-2000 ms for the mCherry probe. FM4-64 was dissolved in $\mathrm{dH}_{2} \mathrm{O}$ at a final concentration of $10 \mu \mathrm{M}$ and added to cells at the moment of nuclear envelope breakdown as described (Kosetsu et al. 2013).

\section{Acknowledgements}

I thank Elysa Overdijk for sharing Sec5 labelled lines and helpful commentary on the manuscript. 


\section{Supplemental figures}

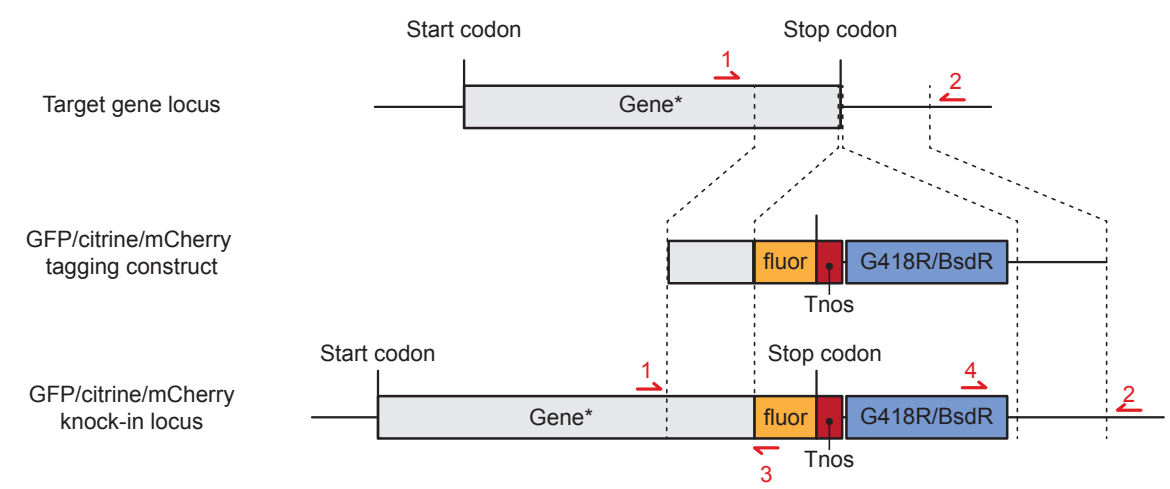

*Sec6

${ }^{*}$ Sec3a

Primers 1+2 $5188 \mathrm{bp}$ (GFP) / $4795 \mathrm{bp}$ (mCh) (2078 bp)

Locus:

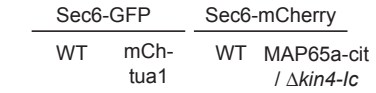

Background:

$6.0 \mathrm{~kb}$
$5.0 \mathrm{~kb}$
$2.0 \mathrm{~kb}$

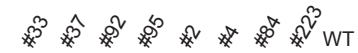

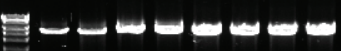

*Sec5b

${ }^{*} \operatorname{Sec} 5 d$

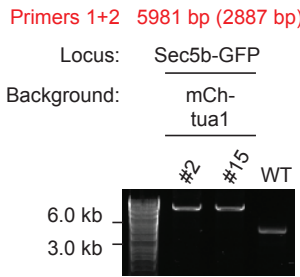

Primers 1+2 5538 bp (2524 bp)

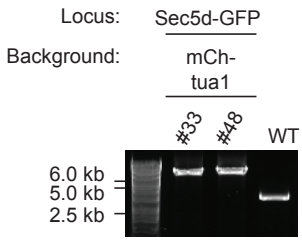

5328 bp (2250 bp)

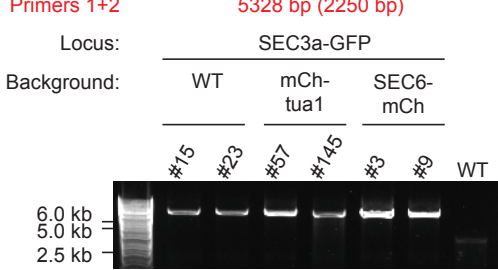

*MAP65c

Primers 1+2 4822 bp (2477 bp)

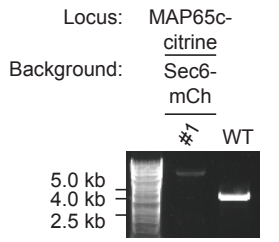

${ }^{*}$ PpKEULE
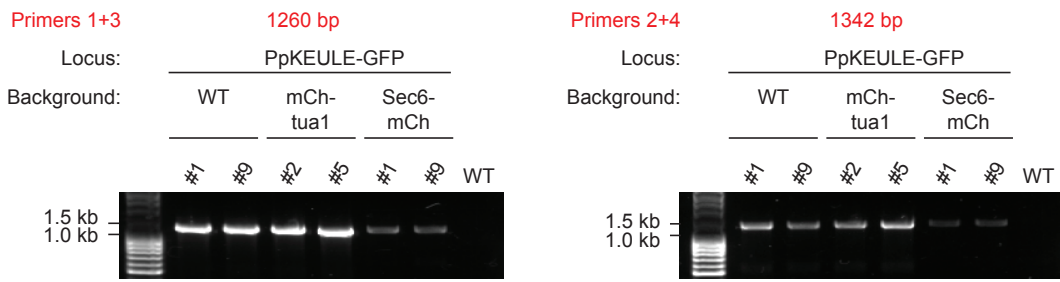

Figure S1 - Generation and verification of fluorophore tagged versions of Sec3a, Sec5b, Sec5d, Sec6 and PpKEULE. Schematic representation of a typical genetic locus and the construct used for C-terminal GFP/citrine/mCherry tagging via homologous recombination (dashed lines; not drawn to scale). In labelled lines, the original stop codon is replaced with a fragment containing a fluorophore encoding sequence (orange box), the nopaline synthase terminator (red box) and a cassette conferring G418 or blasticidin resistance (blue box). Red arrows denote primer binding sites used for confirmation of the obtained lines by PCR. For each indicated 


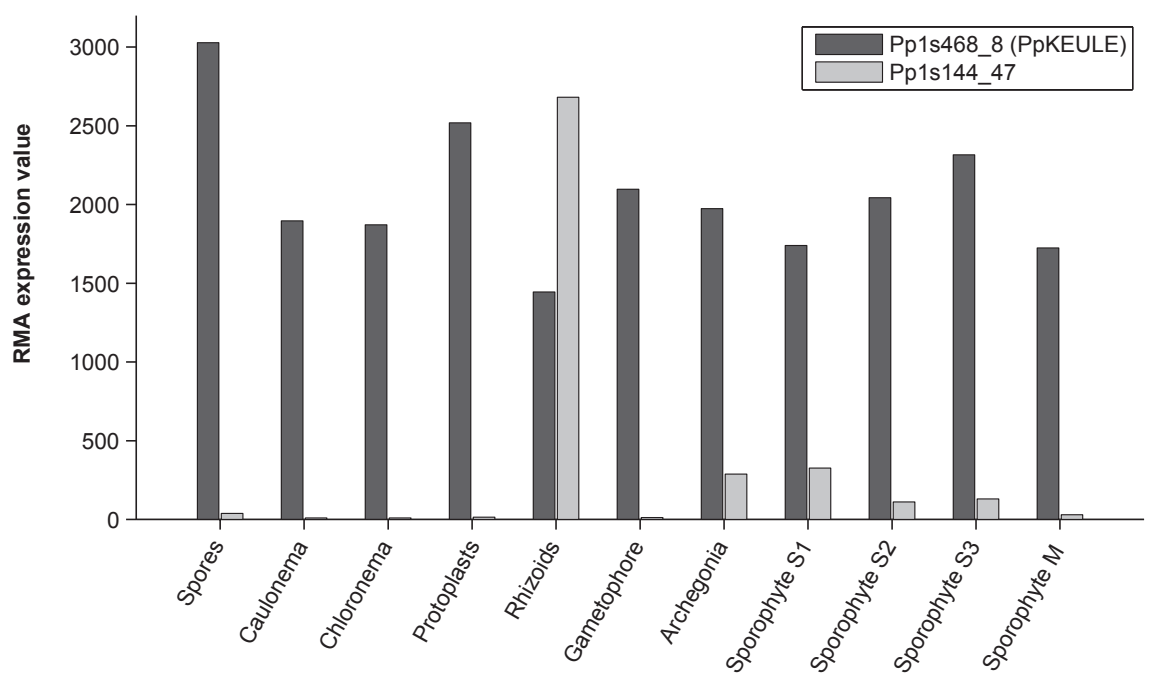

Figure S2 - Expression analysis of two $P$. patens genes encoding Sec1/munc18 family proteins similar to Arabidopsis KEULE. The robust multi-array average (RMA) expression value of two genes encoding KEULE homologs is plotted for various moss tissues/life cycle stages. The locus identifier in version 1.6 of the Physcomitrella genome assembly of these genes is indicated. Expression data was obtained from the publically available Physcomitrella transcriptome atlas (Ortiz-Ramírez et al. 2016).

gene with a knocked-in construct for fluorescent labelling the product obtained after PCR reaction and its predicted size are given. The numbers of two independent transformants and their genetic background are given above the gel images. 


\section{References}

Assaad, F.F. et al., 1996. The KEULE gene is involved in cytokinesis in Arabidopsis. Molecular \& General Genetics, 253(3), pp.267-277.

Boruc, J. \& van Damme, D., 2015. Endomembrane trafficking overarching cell plate formation. Current Opinion in Plant Biology, 28, pp.92-98.

Buschmann, H. \& Zachgo, S., 2016. The evolution of cell division: From streptophyte algae to land plants. Trends in Plant Science, 21(10), pp.872-883.

Cvrčková, F. et al., 2012. Evolution of the land plant exocyst complexes. Frontiers in Plant Science, 3(159).

de Craene, J.-O. et al., 2006. Rtn1p is involved in structuring the cortical endoplasmic reticulum. Molecular Biology of the Cell, 17(7), pp.3009-3020.

van Damme, D., 2009. Division plane determination during plant somatic cytokinesis. Current Opinion in Plant Biology, 12(6), pp.745-751.

Drakakaki, G. et al., 2012. Isolation and proteomic analysis of the SYP61 compartment reveal its role in exocytic trafficking in Arabidopsis. Cell Research, 22(2), pp.413-424.

Drakakaki, G., 2015. Polysaccharide deposition during cytokinesis: Challenges and future perspectives. Plant Science, 236, pp.177-184.

Dubuke, M.L. et al., 2015. The exocyst subunit Sec6 interacts with assembled exocytic SNARE complexes. The Journal of Biological Chemistry, 290(47), pp.28245-28256.

Fendrych, M. et al., 2010. The Arabidopsis exocyst complex is involved in cytokinesis and cell plate maturation. The Plant Cell, 22(9), pp.3053-3065.

Garcia-España, A. et al., 2009. Intron evolution: Testing hypotheses of intron evolution using the phylogenomics of tetraspanins. PLoS one, 4(3), e4680.

Grosshans, B.L., Ortiz, D. \& Novick, P., 2006. Rabs and their effectors: achieving specificity in membrane traffic. Proceedings of the National Academy of Sciences of the United States of America, 103(32), pp.1182111827.

Hála, M. et al., 2008. An exocyst complex functions in plant cell growth in Arabidopsis and tobacco. The Plant Cell, 20(5), pp.1330-1345.

Hashizume, K. et al., 2009. Yeast Sec1p functions before and after vesicle docking. Molecular Biology of the Cell, 20(22), pp.4673-4685.

Heider, M.R. et al., 2015. Subunit connectivity, assembly determinants and architecture of the yeast exocyst complex. Nature Structural \& Molecular Biology, 23(1), pp.59-66.

Hiwatashi, Y. et al., 2008. Kinesins are indispensable for interdigitation of phragmoplast microtubules in the moss Physcomitrella patens. The Plant Cell, 20(11), pp.3094-3106.

Ho, C.-M.K. et al., 2011. Interaction of antiparallel microtubules in the phragmoplast is mediated by the microtubule-associated protein MAP65-3 in Arabidopsis. The Plant Cell, 23(8), pp.2909-2923.

Hong, W. \& Lev, S., 2014. Tethering the assembly of SNARE complexes. Trends in Cell Biology, 24(1), pp.35-43.

James, P., Halladay, J. \& Craig, E.A., 1996. Genomic libraries and a host strain designed for highly efficient two-hybrid selection in yeast. Genetics, 144(4), pp.1425-1436.

Javelle, M. et al., 2011. Genome-wide characterization of the HD-ZIP IV transcription factor family in maize: Preferential expression in the epidermis. Plant Physiology, 157(2), pp.790-803.

Jürgens, G. et al., 2015. Plant cytokinesis: a tale of membrane traffic and fusion. Biochemical Society Transactions, 43(1), pp.73-78.

Karnahl, M. et al., 2017. ER assembly of SNARE complexes mediating formation of partitioning membrane in Arabidopsis cytokinesis. eLife, 6, e25327.

de Keijzer, J. et al., 2017. Shortening of microtubule overlap regions defines membrane delivery sites during plant cytokinesis. Current Biology, 27(4), pp.514-520.

Kosetsu, K. et al., 2013. MICROTUBULE-ASSOCIATED PROTEIN65 is essential for maintenance of phragmoplast bipolarity and formation of the cell plate in Physcomitrella patens. The Plant Cell, 25(11), pp.4479-4492.

Koumandou, V.L. et al., 2007. Control systems for membrane fusion in the ancestral eukaryote; evolution of tethering complexes and SM proteins. BMC Evolutionary Biology, 7, p.29.

Mcmichael, C.M. \& Bednarek, S.Y., 2013. Cytoskeletal and membrane dynamics during higher plant cytokinesis. New Phytologist, 197(2013), pp.1039-1057. 
Morgera, F. et al., 2012. Regulation of exocytosis by the exocyst subunit Sec6 and the SM protein Sec1. Molecular Biology of the Cell, 23(2), pp.337-346.

Müller, S. \& Jürgens, G., 2015. Plant cytokinesis - No ring, no constriction but centrifugal construction of the partitioning membrane. Seminars in Cell and Developmental Biology, 53, pp.10-18.

Müller, S., Wright, A.J. \& Smith, L.G., 2009. Division plane control in plants: new players in the band. Trends in Cell Biology, 19(4), pp.180-188.

Munson, M. \& Novick, P., 2006. The exocyst defrocked, a framework of rods revealed. Nature Structural \& Molecular Biology, 13(7), pp.577-581.

Nishiyama, T. et al., 2000. Tagged mutagenesis and gene-trap in the moss, Physcomitrella patens by shuttle mutagenesis. DNA Research, 7(1), pp.9-17.

Ortiz-Ramírez, C. et al., 2016. A transcriptome atlas of Physcomitrella patens provides insights into the evolution and development of land plants. Molecular Plant, 9(2), pp.205-220.

Park, M. et al., 2012. Sec1/Munc18 protein stabilizes fusion-competent syntaxin for membrane fusion in Arabidopsis cytokinesis. Developmental Cell, 22(5), pp.989-1000.

Paumet, F., Rahimian, V. \& Rothman, J.E., 2004. The specificity of SNARE-dependent fusion is encoded in the SNARE motif. Proceedings of the National Academy of Sciences of the United States of America Sciences, 101(10), pp.3376-3380.

Qi, X. et al., 2011. A specific role for Arabidopsis TRAPPII in post-Golgi trafficking that is crucial for cytokinesis and cell polarity. The Plant Journal, 68(2), pp.234-248.

Rensing, S.A. et al., 2005. Protein encoding genes in an ancient plant: analysis of codon usage, retained genes and splice sites in a moss, Physcomitrella patens. BMC Genomics, 6(1), p.43.

Richter, S. et al., 2014. Delivery of endocytosed proteins to the cell-division plane requires change of pathway from recycling to secretion. eLife, 3, e02131.

Rogozin, I.B. et al., 2003. Remarkable interkingdom conservation of intron positions and massive, lineagespecific intron loss and gain in eukaryotic evolution. Current Biology, 13(17), pp.1512-1517.

Rybak, K. et al., 2014. Plant cytokinesis is orchestrated by the sequential action of the TRAPPII and exocyst tethering complexes. Developmental Cell, 29(5), pp.607-620.

Schmiedel, G., Reiss, H.D. \& Schnepf, E., 1981. Associations between membranes and microtubules during mitosis and cytokinesis in caulonema tip cells of the moss Funaria hygrometrica. Protoplasma, 108(1-2), pp.173-190.

Sivaram, M.V.S. et al., 2005. Dimerization of the exocyst protein Sec6p and its interaction with the t-SNARE Sec9p. Biochemistry, 44(16), pp.6302-6211.

Sivaram, M.V.S. et al., 2006. The structure of the exocyst subunit Sec6p defines a conserved architecture with diverse roles. Nature Structural \& Molecular Biology, 13(6), pp.555-556.

Söllner, T. et al., 1993. SNAP receptors implicated in vesicle targeting and fusion. Nature, 362(6418), pp.318-324.

Steiner, A. et al., 2016. The membrane-associated Sec1/Munc18 KEULE is required for phragmoplast microtubule reorganization during cytokinesis in Arabidopsis. Molecular Plant, 9(4), pp.528-540.

Stenmark, H., 2009. Rab GTPases as coordinators of vesicle traffic. Nature Reviens: Molecular Cell Biology, 10(8), pp.513-525.

Stöckle, D. et al., 2016. Putative RopGAPs impact division plane selection and interact with kinesin-12 POK1. Nature Plants, 2(9), e16120.

Südhof, T.C. \& Rothman, J.E., 2009. Membrane fusion: grappling with SNARE and SM proteins. Science, 323(5913), pp.474-477.

Thellmann, M. et al., 2010. Tethering factors required for cytokinesis in Arabidopsis. Plant Physiology, 154(2), pp. $720-732$.

Vukašinović, N. \& Žárský, V., 2016. Tethering complexes in the Arabidopsis endomembrane system. Frontiers in Cell and Developmental Biology, 4, p.46.

Wickner, W. \& Schekman, R., 2008. Membrane fusion. Nature Structural \& Molecular Biology, 15(7), pp.658664.

Wu, J. et al., 2013. Regulation of cytokinesis by exocyst subunit SEC6 and KEULE in Arabidopsis thaliana. Molecular Plant, 6(6), pp.1863-1876.

Yamada, M., Miki, T. \& Goshima, G., 2016. Imaging mitosis in the moss Physcomitrella patens. In P. Chang \& R. Ohi, eds. Methods in Molecular Biology (Clifton, N.J.). New York, NY, United States: Springer New York, pp. 263-282. 
Yu, I.-M. \& Hughson, F.M., 2010. Tethering factors as organizers of intracellular vesicular traffic. Annual Review of Cell and Developmental Biology, 26(1), pp.137-156.

Zhang, Y. et al., 2013. The Arabidopsis exocyst subunit SEC3A is essential for embryo development and accumulates in transient puncta at the plasma membrane. New Phytologist, 199(1), pp.74-88. 


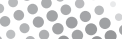

$\therefore \% 88 \%$

::898898.

88.

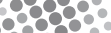

Chapter 4 - Differential regulation of $\therefore$ microtubule flux in the spindle and $\because$ phragmoplast involves kinesin-4i. mediated regulation of microtubule polymerization

Jeroen de Keijzer, Chaniël Bakker and Marcel E. Janson

Laboratory of Cell Biology, Wageningen University, Droevendaalsesteeg 1, 6708 PB

Wageningen, The Netherlands

"Reality is captured in the categorical nets of language only at the expense of fatal distortion"

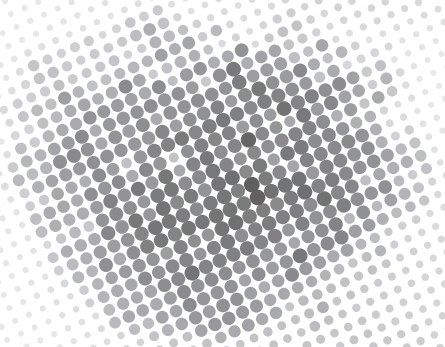

- Friedrich Nietzsche 


\section{Abstract}

Plant cytokinesis relies on the construction of a division plane that is brought about by the bipolar phragmoplast microtubule array. The microtubules of the apparatus dynamically reorganize during expansion, whereby bipolarity is maintained and a steady length control is achieved. Since plant cells lack defined microtubule organizing centres, the organisation of this array is understood to be the collective result of several dispersed activities. Activities driving phragmoplast array architecture have been proposed to include retrograde flux of microtubule polymers and localized regulation of microtubule dynamics. It is unclear however if and how these mechanisms are coupled for efficient length control. Microtubules of opposite polarity in the phragmoplast centre are able to form regions of overlap that have a short, defined length. We previously established that the selective inhibition of plus-end growth by kinesin-4 type molecular motors can limit the length of overlaps. In this work we explore if kinesin-4 mediated control of plus end growth at overlaps determines the rate of retrograde microtubule flux in the moss Physcomitrella patens. First we establish in dividing moss cells that kinesin-4s are indeed negative regulators of microtubule plus-end dynamics at the zone of overlap. We then show using localized marking of microtubule segments by photo-activation that in absence of kinesin-4 activity flux rates are enhanced, ultimately resulting in elongation of the entire apparatus. We further uncover that microtubule growth and flux behaviour are differentially tuned in the metaphase spindle and phragmoplast apparatus. Overall, we find that the balance between growth in the overlap, microtubule flux and potentially minus-end breakdown at the poles influences both the size of the overlap itself as well as the length of the entire division apparatus.

\section{Introduction}

The plant phragmoplast is a bipolar, microtubule-based structure that develops from the spindle after chromosome segregation and facilitates biogenesis of a dividing wall. Unique among different modes of eukaryotic cytokinesis, construction of this dividing wall occurs via a transient membrane compartment termed the cell plate, that is initiated in the cell centre and expands towards the parental plasma membrane. The radial expansion of the cell plate requires a concerted reshaping of the phragmoplast. To this end, phragmoplast microtubules are continually added and reorganized at the margins of the expanding cell plate (Murata et al. 2013; reviewed in Lee \& Liu 2013). Given this dynamic nature of the plant cytokinetic microtubule array and the general lack of defined microtubule-organizing centres in land plants, the phragmoplast is an intriguing example to study the (self-)organizing principles that build acentrosomal bipolar microtubule arrays.

One aspect of array architecture particularly important for the phragmoplast is the length of the structure. Why does length matter for phragmoplast functioning? In the first place, the rigid outer wall of plant cells imposes restrictions on the possible orientations the division apparatus can have within a cell. Yet, plant cells often require a flexible adaptation of the division plane to contribute to tissue formation. Reorientation and shortening of the division apparatus would be essential to obtain this flexibility, as is indeed observed in small cells realizing a transverse division plane (Palevitz 1993; de Ruijter et al. 1997). Secondly, phragmoplast microtubules have a tentative function in transport of cell plate destined vesicles (Otegui et al. 2001; Lee et al. 2001). Since the transport properties of motor-propelled cargoes generally depends on microtubule 
network topology (Ando et al. 2015), a well-tuned length of the phragmoplast microtubule network could facilitate efficient delivery of cell plate material. Finally, recent findings indicate that active conservation of the length scale of the apparatus is important during phragmoplast expansion (Murata et al. 2013).

Many ingredients for efficient length control of bipolar microtubule structures and insight into their interplay have been obtained from work on animal spindles (reviewed in Goshima \& Scholey 2010). A major phenomenon recurrently encountered across different cell types involves the poleward translocation of microtubules (termed microtubule flux; Sawin \& Mitchison 1991). New polymer mass to fuel this activity is typically created through polymerization at plus ends pointing inwards and its removal is achieved through breakdown of microtubules at the poles, often at their minus ends (reviewed in Gadde \& Heald 2004; Petry 2016). The balance between rates of these activities is able to set a steady-state length of the apparatus (Shirasu-Hiza et al. 2004; Wang et al. 2010; Loughlin et al. 2011; Reber et al. 2013). Transition to a different regime through alteration of one parameter (for example decreasing the rate of minus-end breakdown), can effectuate elongation or shortening, as was shown for example during anaphase B spindle elongation in fruit fly mitosis (Wang et al. 2013). However, in both human and fly mitosis spindle length regulation is not dependent on flux per se, but additionally appears to be linked to spindle microtubule dynamics via mechanisms not functioning through poleward microtubule translocation (Ganem et al. 2005; Buster et al. 2007). Nevertheless, this dispensability of flux is likely related to presence of a discrete microtubule organizing centre, since in acentrosomal oocyte spindles flux appears to be the major driver for spindle bipolarization and size determination (Gadde \& Heald 2004; Mitchison 2005).

Interestingly, the forces driving spindle microtubule flux are thought to be produced in between a pair of antiparallel microtubules from opposite poles (Nislow et al. 1992; Goshima \& Scholey 2010). This also means that control over growth processes taking place here could be directly translated to how much polymer is available for translocation, and thus affect flux rates. Sites of microtubule overlap could therefore be the location where sliding action and plus end growth are coupled to help determine the overall length of a bipolar structure. Intriguingly, short regions of antiparallel overlap are abundantly found in the centre of the plant phragmoplast (Hepler \& Jackson 1968; Hiwatashi et al. 2008; Ho et al. 2011), which is also the location containing the majority of microtubule plus-ends (Vantard et al. 1990; Ho et al. 2011). The anaphase spindle forms the basis of the plant phragmoplast, as well as the animal central spindle. Flux is less well studied in these cytokinetic microtubule networks, although they probably have profoundly different design criteria than the preceding spindle. By characterizing the transition from the mitotic to the cytokinetic state of the network, we hope to learn more about the regulation of flux. Pole-ward microtubule translocation has been observed in the phragmoplast (Asada et al. 1991; Murata et al. 2013). However, if and how pole-ward flux is linked to regulation of microtubule dynamic instability within the antiparallel overlap geometry remains unclear. To investigate this we combined live-cell imaging of marked microtubule segments with deletion of a microtubule growth inhibitor functioning at phragmoplast microtubule overlaps in the moss plant Physcomitrella patens (de Keijzer et al. 2017). We demonstrate that flux rates in the phragmoplast rise with an increase of growth activity at the zone of microtubule overlap and that this in turn elongates the entire apparatus. The here unravelled coupling between microtubule growth at overlaps and flux behaviour not only leads to better understanding of phragmoplast length control, but also expands our knowledge on how overlap-shortening is achieved for spatially confined cell plate assembly. 
A
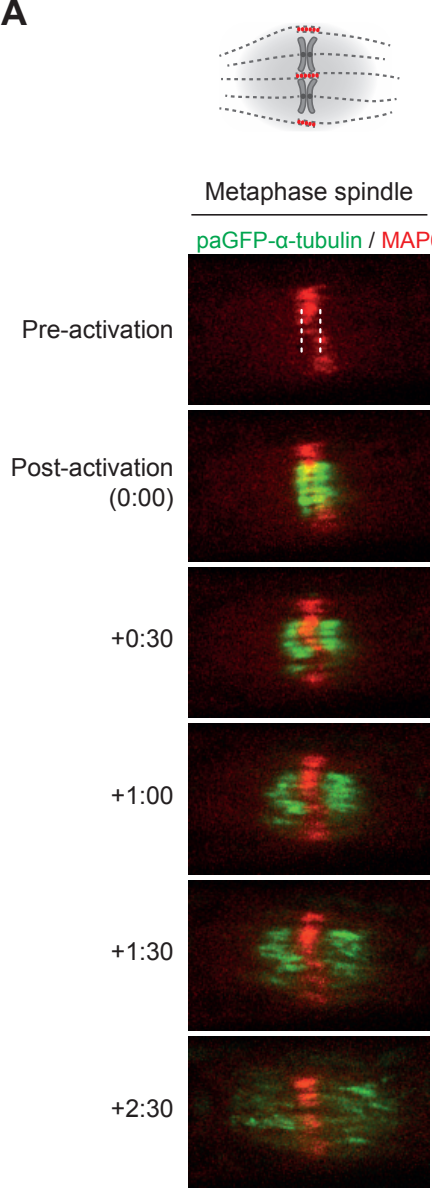

$+5: 00$

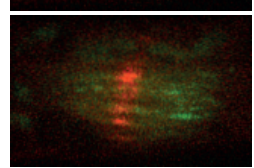

B

\section{Average \\ kymograph of entire activated zone}

\section{Kymograph of} segment of activated zone

$$
\text { paGFP-a-tubulin }
$$

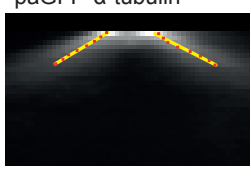

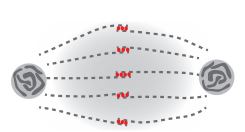

Phragmoplast initiation
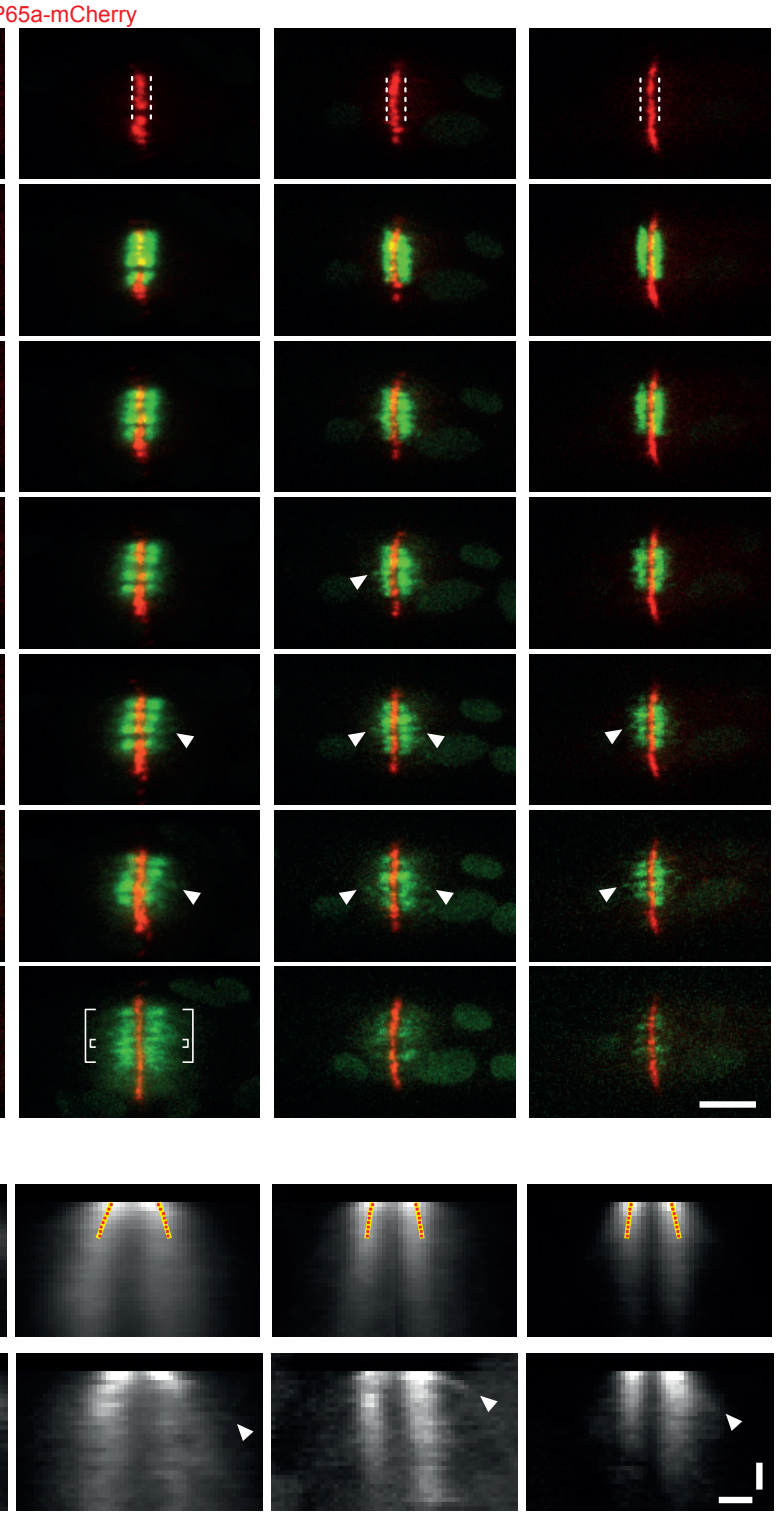

Figure 1 - Rapid, synchronized retrograde microtubule flux behaviour in the metaphase spindle is succeeded by nonsynchronous movement during cytokinesis. (A) Time lapse of a photo-activation experiment performed in cells expressing both MAP65a-mCherry and paGFP- $\alpha$-tubulin at four stages of cell division (illustrated at the top). Two $4 \mu \mathrm{m}$ long regions at opposite sides of the MAP65-labeled spindle/phragmoplast midplane were irradiated by $405 \mathrm{~nm}$ wavelength 


\section{Results and discussion}

While retrograde microtubule flux in the phragmoplast has been documented in several studies, methods to visualize the phenomenon relied on pulsed introduction of labelled tubulin through cell permeabilization (Asada et al. 1991; Asada et al. 1997) or on motility analysis of individual microtubule speckles and gamma tubulin spots (Murata et al. 2013). To more comprehensively capture flux behaviour in a non-invasive and quantifiable way, we used localized activation of a photo-activatable probe incorporated in the microtubule polymers. To this end we generated a moss line expressing photo-activatable GFP (paGFP) fused to moss $\alpha$-tubulin (Patterson \& Lippincott-Schwartz 2002; Hiwatashi et al. 2008). We subsequently fused red fluorophore mCherry to the conserved bundling protein MAP65 to mark the zone where microtubules of the opposite poles interdigitate to form overlaps (Figure S1B) (Kosetsu et al. 2013). Expression of neither probe resulted in aberrant colony morphology or growth deficiency compared to wild-type (Figure S1A). We then used this strain to study flux behaviour during 3 subsequent stages of phragmoplast development (initiation of the phragmoplast, solid phragmoplast and ring-shaped phragmoplast) and also during metaphase to understand if flux behaviour changed during the spindle-to-phragmoplast transition (See materials and methods for a full definition of the used stages). Labelled tubulin on either side of the MAP65-labelled interdigitate was locally photo-activated after which the redistribution of marked microtubule segments was traced for about 5 minutes (Figure 1A). By performing a similar activation experiment on the largely static array of interphase non-apical cells using identical acquisition parameters we established that photo-bleaching of activated paGFP was not a relevant factor on the timescale flux experiments were performed (Figure S1C-E). Thus, the observed gradual dimming of photo-activated polymer mass during cell division can in large be attributed to polymer turnover.

During the phragmoplast stages microtubule translocation behaviour was markedly different from mitosis. In the metaphase spindle marked microtubule segments collectively moved towards the poles (Figure 1A). The majority of activated phragmoplast microtubules remained largely immobile. Incidentally individual microtubule segments emanated from the largely static pool and moved relatively fast towards the poles (Figure 1A, arrowheads). To quantify the overall displacement behaviour of activated zones (here referred to as bulk flux rate), kymographs capturing the average redistribution of fluorescence after photo-activation were generated and used to derive flux speeds (Figure 1B). This revealed that the mean bulk flux rate of about $1.5 \mu \mathrm{m} / \mathrm{min}$ prevalent during metaphase rapidly declined during phragmoplast initiation to end up approximately an order of magnitude lower during the later phragmoplast stages (Figure 2A). To measure the speeds of the fast-moving microtubule segments, kymographs of a small portion of the activated area were made and used to determine the maximum displacement rate

light (indicated by the dashed lines) after which movement of the activated region was monitored. Time is indicated in min:seconds. The arrowheads indicate examples of occasional fast retrograde movement of individual microtubule segments in the phragmoplast. The two bracket pairs illustrate the regions used to generate the two kymograph types depicted in B. Pictures are a maximum z-projection of 3 planes spaced $0.5 \mu \mathrm{m}$ apart. Scalebar, $5 \mu \mathrm{m}$. (B) Kymographs representing microtubule flux behaviour in the division apparatus. The overall retrograde movement is illustrated by kymographs averaging the entire activated area (top row). Here, intensity-weighted centres were computationally determined (red marks), after which displacement speed was estimated through linear regression (yellow line). The kymographs created from a small segment of the activated area (bottom row) capture the occasional retrograde microtubule movement as diagonal lines originating from the activated bulk (marked with arrowheads). Horizontal scalebar, $1 \mu \mathrm{m}$; vertical scalebar, $1 \mathrm{~min}$. 
of discrete microtubule segments (Figure 1B, arrowheads). These speeds were approximately equal to the rates of synchronized flux in the metaphase spindle, with a tendency to decrease as cytokinesis progressed (Figure 2B). It thus appears that among phragmoplast microtubules only a subset undergoes poleward transport at rates potentially relevant for biological function. These observations closely resemble the behaviour and even the speeds described for flux in the phragmoplast of tobacco culture cells (Murata et al. 2013). These authors ascribe the biphasic nature of phragmoplast microtubule flux velocities to the presence of a population of stabilized, parallel bundled microtubules incapable of flux, and a separate dynamic population which exhibits flux. Similarly, heterogeneous flux behaviour in spindles from Xenopus egg extracts was attributed to the different interactions fluxing microtubules had with these of the same and the opposite pole (Yang et al. 2008). To better understand what underlies differential flux behaviour in the plant phragmoplast, it will be valuable to identify the proteins that drive flux. While several lines of research suggest that, as in the animal spindle, this role is fulfilled by tetrameric kinesin-5 motors (Asada et al. 1997; Bannigan et al. 2007; Gillmor et al. 2016), also kinesin-12 and kinesin-14 motor proteins have been implicated (Murata et al. 2013; Lee \& Liu 2013). Nonetheless, we conclude that flux is a recurring phenomenon in the phragmoplast of both a higher and a lower plant and could hence be an important and universal signature of length control.

Having established the flux behaviour in the division apparatus of $P$. patens we next investigated if there could be a dependency on microtubule growth occurring at the antiparallel overlaps in the phragmoplast centre. In a previous study we identified two kinesin- 4 type molecular motors

A

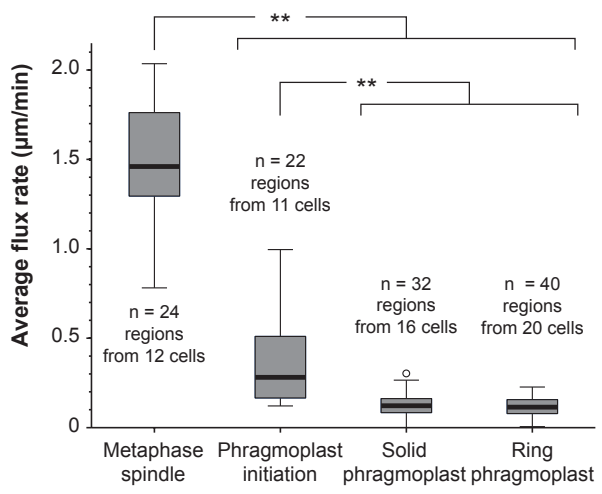

B

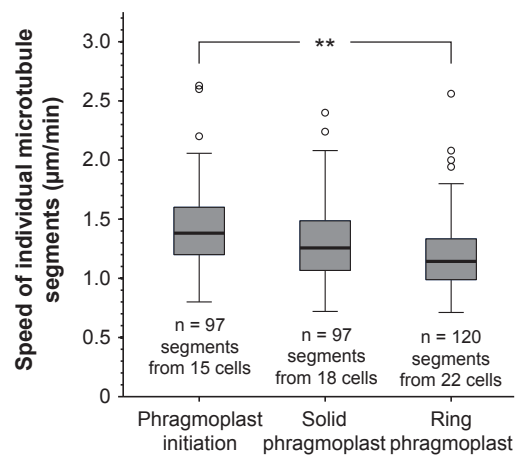

Figure 2 - Quantification of bulk microtubule flux rate and maximum speed of individual highlighted microtubule segments during four stages of cell division. (A) Boxplots of the bulk retrograde microtubule flux rate during four stages of cell division. Flux rates were determined using the kymograph analysis method illustrated in figure $1 \mathrm{~B}$ for the indicated number of activated regions. The whiskers indicate minimum / maximum observed values or 1.5 times the interquartile range when outliers (circles) were present. Flux rates during the different stages were all significantly different from each other $\left({ }^{* *} \mathrm{P}<0.001\right)$ except the ring and solid phragmoplast stages in a one-way ANOVA with Tamhane T2 post-hoc test. (B) Boxplots of the maximum speed of individual highlighted microtubule segments during four stages of cell division. Speeds were manually measured from kymographs of a segment of the activated zone as shown in figure 1B for the indicated number of microtubule segments. The whiskers indicate minimum / maximum observed values or 1.5 times the interquartile range when outliers (circles) were present. Differences in speeds found to be significant in a Kruskal-Wallis test with pairwise comparisons are highlighted $\left({ }^{* *} \mathrm{P}<0.001\right)$. 
that jointly prevented elongation of phragmoplast overlaps (Kin4-Ia and Kin4-Ic, hereafter collectively referred to as kinesin-4; de Keijzer et al. 2017). Overlap shortening can be caused by plus-end-directed sliding activity or growth-inhibiting properties attributed to kinesin-4 family proteins. Using an in vitro assay we determined that moss kinesin-4 indeed possesses such polymerization inhibiting properties. Interestingly, kinesin-4 is absent from metaphase spindle microtubules with recruitment to overlaps occurring rapidly at anaphase onset, the same moment we observed bulk flux velocities to drop. This concurrent switch in behaviour makes kinesin-4 an interesting candidate for being part of the mechanism causing flux velocities to fall as the mitotic microtubule array is succeeded by the cytokinetic array. However, kinesin-4 recruitment and differential flux behaviour could also be uncoupled processes that happen to share the same temporal regulation. Functional analysis of kinesin-4 could thus also give insight into the mechanisms of differential flux regulation. First, we assessed whether changed overlap dynamics in absence of kinesin- 4 can be attributed to altered microtubule dynamics in vivo. For this, we visualized length dynamics of MAP65-labelled overlaps in wild-type and kinesin-4 knock-out cells simultaneously with the microtubule plus-end marker EB1b-mCherry (Figure S2A-B) (Hiwatashi et al. 2014). Compared to wild-type, events in which overlap length suddenly extended occurred over three times more frequently in cells lacking kinesin-4 (Figure S2C). Most crucially, this increase was almost exclusively due to a rise in overlap extension events that were preceded by EB1b-mCherry comets, showing that kinesin- 4 supresses overlap extension by inhibiting outgrowth of microtubule plus ends located within overlaps. Moss lines lacking these motors therefore enabled us to address the relation between microtubule polymerization in overlaps and flux.

To see if the established enhancement of growth at the microtubule interdigitate in kinesin-4 knock-out cells increases flux rates, we established moss lines in which the overlap was visible and microtubule photo-activation experiments could be performed. We first tested the use of the red photo-activatable fluorophore pamCherry1 (Subach et al. 2009; hereafter referred to as pamCherry) for flux experiments, since it is compatible with the emission spectrum of the MAP65a-citrine probe present in the previously generated kinesin- 4 knock-out mutants. Expression of pamCherry- $\alpha$-tubulin, like the before-mentioned paGFP-based probe, did not alter moss growth rates and colony morphology in both wild-type and kinesin- 4 knock-out lines (data not shown). Although photo-stability and brightness of pamCherry were considerably reduced compared to paGFP (Figure S3A-B; cf. Figure S1D-E), limiting its use to visualize individual microtubule segments, bulk flux could be determined well in all previously used stages of cell division. Flux experiments using pamCherry in wild-type and kinesin-4 knock-out cells revealed that retrograde movement of microtubules appeared to occur faster in kinesin-4 knock-out lines (Figure 3A). Capturing movement using kymographs of the average intensity redistribution after photo-activation, allowed us to determine that bulk flux rates were slightly enhanced during metaphase and at least doubled throughout cytokinesis in kinesin-4 knockout cells when compared to wild-type (Figure 3B-C). The down-regulation of flux during the spindle-to-phragmoplast transition was preserved in kinesin- 4 knock-out cells, indicating that other factors besides kinesin- 4 contribute to this temporal switch in flux behaviour. Nonetheless, the sole absence of kinesin-4 already significantly enhanced phragmoplast microtubule flux rates. While this could be directly attributed to a lack of polymerization inhibiting activity by kinensin-4, an indirect effect of cell plate assembly, which is hampered in the kinesin-4 mutant, on microtubule activities in overlaps could also be envisaged (de Keijzer et al. 2017). However, since during the phragmoplast initiation stage cell plate membrane accumulation is still minimal 
(Kosetsu et al. 2013; de Keijzer et al. 2017), yet here flux markedly increases (Figure 3A, arrowheads; Figure 3B) we deem a direct relationship most plausible. Whether enhanced speed and/or frequency of individual, fast-moving microtubule segments accounts for the increased

A

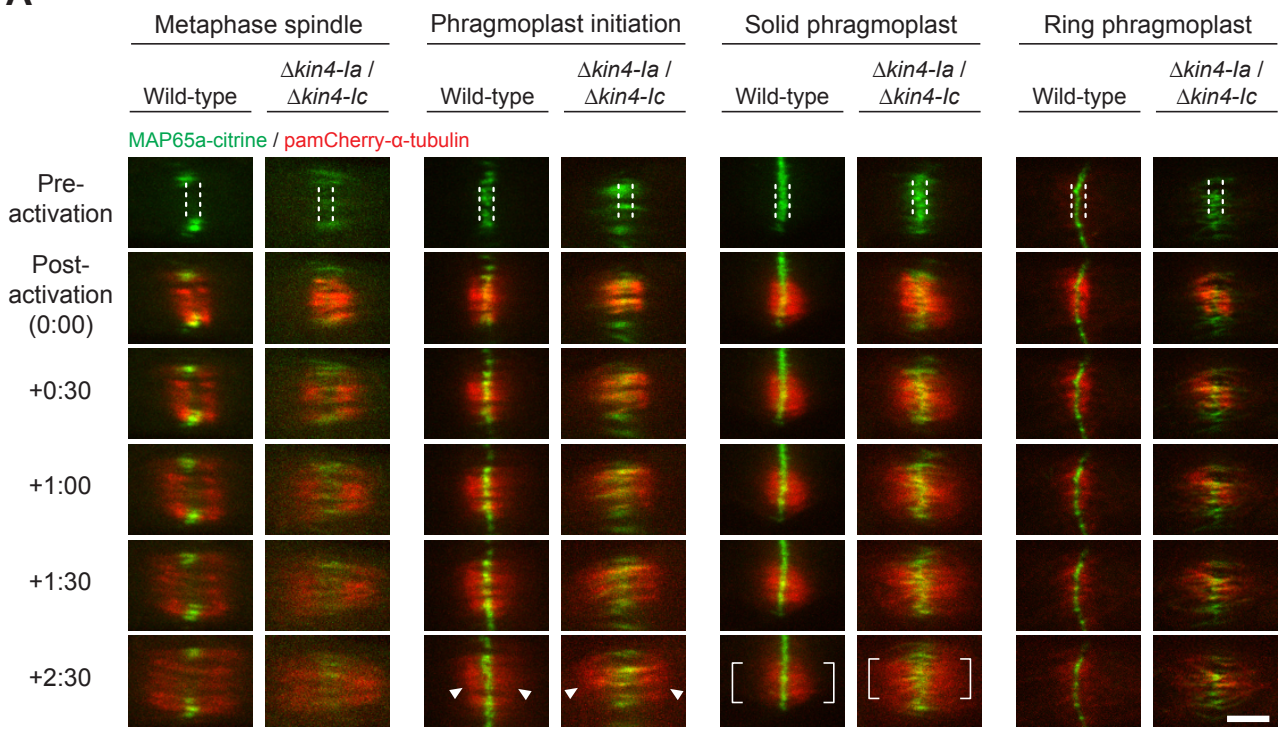

B

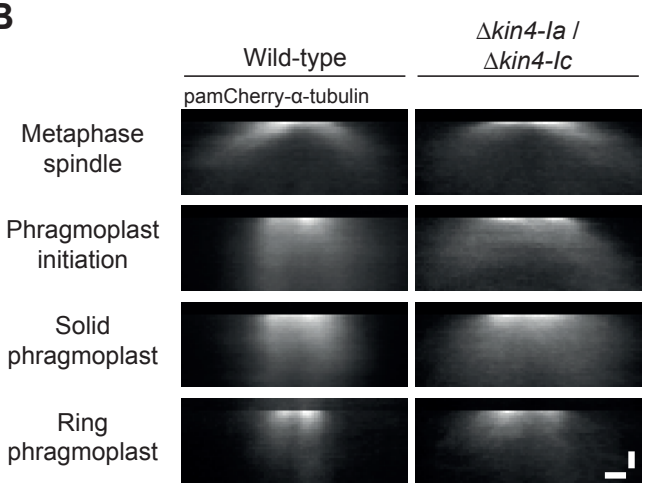

C

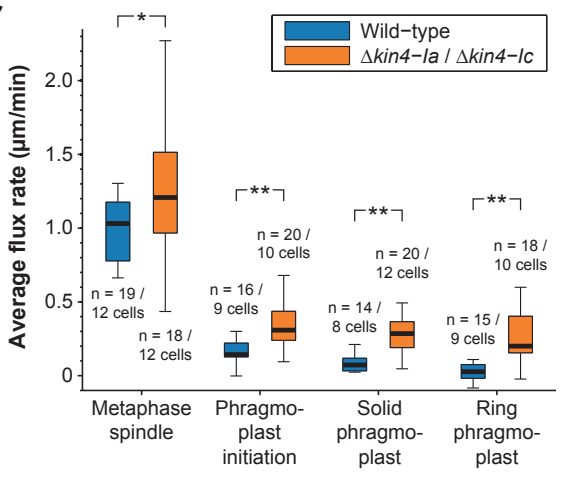

Figure 3 - Kinesin-4 mediated limitation of microtubule overlap length reduces phragmoplast flux rates. (A) Time lapse of a photo-activation experiment performed in wild-type and $\Delta k i n 4-l a$ / $\Delta$ kin4-Ic knock-out cells expressing MAP65a-citrine and pamCherry-a-tubulin at four stages of cell division. Two $4 \mu \mathrm{m}$ long regions at opposite sides of the MAP65-labeled spindle/phragmoplast midplane were irradiated by $405 \mathrm{~nm}$ wavelength light (indicated by the dashed lines) after which movement of the activated region was monitored. Time is indicated in min:seconds. The arrowheads highlight an example of larger retrograde displacement of photo-activated microtubules in absence of kinesin-4. The brackets illustrate the region used to generate the kymographs depicted in B. Pictures are a maximum z-projection of 3 planes spaced $0.5 \mu \mathrm{m}$ apart. Scalebar, $5 \mu \mathrm{m}$. (B) Kymographs representing bulk microtubule flux behaviour in the division apparatus of a wild-type and $\Delta$ kin4-la / $\Delta k i n 4-l c$ knock-out cell at four subsequent stages of cell division. Horizontal scalebar, $1 \mu \mathrm{m}$; vertical scalebar, $1 \mathrm{~min}$. (C) Boxplots of the bulk retrograde microtubule flux rate during four stages of cell division in wild-type and kinesin- 4 knock out cells. Flux rates were determined using the kymograph analysis method illustrated in figure $1 \mathrm{~B}$ for the indicated number of activated regions. The whiskers indicate minimum / maximum observed values. Differences between the two genotypes within each cell division stage were evaluated with Students $t$-test assuming unequal variances $\left({ }^{*} P<0.05\right.$ and ${ }^{* *} \mathrm{P}<0.001$ ). 
bulk flux rates upon kinesin-4 loss could not be established due to the less discrete photoactivation and reduced brightness of the pamCherry- $\alpha$-tubulin probe.

Taken together, our results show that lifting inhibition of microtubule polymerization at overlaps through kinesin-4 removal enhances the rate of retrograde translocation of microtubule polymer mass. This suggests that in the wild-type phragmoplast the full potential of microtubule translocation (assuming that it is generated through activity of mechanochemical enzymes; Asada et al. 1997) is not realized due to a lack of substrate polymers (i.e. there is an flux 'overcapacity'). Such an excess in flux capacity is potentially used to shorten overlaps at the onset of cytokinesis (de Keijzer et al. 2017). This scenario could also explain why flux rates during phragmoplast initiation are higher than during later stages (Figure 2A): at this early stage there is still polymer available within the long overlaps inherited from the spindle apparatus to drive flux. The presence of a flux overcapacity also hints that there are likely mechanisms in place that prevent overlapping microtubules from sliding entirely apart. This prevention could be supplied by crowding of cross-linking proteins within the overlap, a property described for MAP65-homologue Ase1p (Braun et al. 2011), or could stem from other mechanisms generating feedback between sliding activity and overlap length, such as motor antagonism or directional switching of motors (Subramanian \& Kapoor 2012; Bowne-Anderson et al. 2015). In any case, plus end growth is an important factor in setting the rate of microtubule flux and the concerted action of sliding and growth could keep the antiparallel interaction zone between microtubules at a defined length.

In human cells the pole-to-pole length of the central spindle increased upon removal of kinesin-4 homologue KIF4 (Hu et al. 2011). Our finding that rates of poleward microtubule translocation are enhanced upon loss of kinesin-4 function forms a possible explanation for this observation. To further explore if flux behaviour has consequences for the pole-to-pole length of the entire cell division apparatus we studied length dynamics in more detail. To this end, we used expression of mCherry- $\alpha$-tubulin to visualize the division apparatus throughout cell division in wild-type and kinesin-4 knock-out cells and computationally determined the evolution of pole-to-pole length (Figure4; Figure S3C). Since the two kinesin-4 motors responsible for phragmoplast overlap length control are active during different stages of cytokinesis (Kin4-Ic mainly early, Kin4-Ia only later; de Keijzer et al. 2017), single $\Delta$ kin4-Ia and $\Delta$ kin4-Ic mutants were also analysed. In the wild-type situation apparatus length was rather stable in metaphase and briefly spiked during anaphase after which it shortened steadily during cytokinesis. Shortening of the phragmoplast occurred initially at a pace of about $1 \mu \mathrm{m} /$ minute, to then slowly level of after 30 minutes, yielding an apparatus approximately 60\% shorter than at the start (Figure 4B). In absence of both kinesin-4s pole-to-pole distances increased for both the metaphase spindle and phragmoplast with the length of late phragmoplasts being reduced only by about $20 \%$. The knock-out of kinesin-4s did not alter the overall architecture of the division apparatus (Figure 4A). Interestingly, lengths in the $\Delta$ kint-Ic single mutant closely resembled the double mutant early in mitosis, to then transition to wild-type-like during late cytokinesis (Figure 4A). Through the course of cytokinesis $\Delta$ kin 4 -Ic phragmoplasts shortened from typical kinesin-4 double knock-out lengths to almost wild-type lengths (Figure 4B). This highlights that increased phragmoplast length in absence of kinesin- $4 \mathrm{~s}$ is not just a consequence of the spindle already being longer, but rather makes a case that the phragmoplast system can adapt length by regulation of growth activity at the overlaps (in this case through the later recruitment of Kin4-Ia). Future flux measurements performed in the $\Delta$ kint-Ic single mutant could substantiate this notion. The average length of the division apparatus in the single $\Delta k i n 4-I a$ mutant was indistinguishable 
from that of wild-type. This could be attributed to the very minor effects its sole removal has on overlap lengths (Figure 4A) (de Keijzer et al. 2017). This also showed that the metaphase spindle elongation observed in the double mutant was caused by the absence of Kin4-Ic. Since at this stage in cell division Kin4-Ic localizes to chromosomes (Miki et al. 2014), it is likely that it exerts its inhibition of microtubule growth at this location. This would be in agreement with the behaviour described for human chromokinesin KIF4A, although here its removal downregulated flux (Wandke et al. 2012). The absence of KIF4A was shown to disturb multiple aspects of chromosome alignment and segregation, which could manifest itself differentially on spindle flux in plant and animal cells.

If the constant metaphase spindle length is maintained by a flux rate of 1.5 micron/minute and an equal rate of microtubule depolymerisation at the poles, rapid phragmoplast shortening may be expected at the moment flux is almost completely shut down upon entry into cytokinesis. Whilst initial shortening is indeed fast, the shortening rate decreases slowly. At the poles of animal spindles several breakdown processes relevant for setting spindle length are spatially controlled through a phosphorylation gradient originating from the spindle centre (Loughlin et al. 2011; Uehara et al. 2013). Based on this and findings pointing towards active microtubule breakdown at phragmoplast poles (Panteris et al. 2011; Komis et al. 2017), it is tempting to speculate that minus end depolymerization in the phragmoplast is similarly regulated by an activating gradient that decreases in strength away from the division plane. This form of spatial feedback would lead to a gradual abolishment of the mismatch between depolymerization and polymerization speeds, ultimately causing stabilization of phragmoplast length. Such a mechanism would explain the observed phragmoplast shortening behaviour of the wild-type phragmoplast. Since the higher flux rates in kinesin-4 knock-out cells imply that here the polymerization/depolymerization mismatch is bigger than in wild-type, the same scenario can also explain why shortening occurs only to a limited extend in these cells (Figure 5). It will be interesting to find out if a depolymerization mechanism spatially organized from the division plane indeed provides a comprehensive mean for phragmoplast length determination.

We observed a marked increase in retrograde microtubule flux rates in absence of kinesin-4 mediated growth control at phragmoplast overlaps. Yet, flux rates were not restored to rates that prevailed in the metaphase spindle apparatus minutes earlier (Figure 3C). The inhibition of polymerisation rates by kinesin- 4 is hence not the only aspect of network dynamics altered at the moment of anaphase onset to diminish microtubule flux (Figure 5, right hand side). Thus, overall, setting correct overlap and phragmoplast length at the onset of spindle-to-phragmoplast transition seems to involve an orchestrated downregulation of the capacity to flux, microtubule growth at overlaps and the rate of minus-end depolymerisation (Figure 5). This transition of regimes combined with the outspoken role of overlap-length shortening for cell plate assembly, makes the plant phragmoplast an interesting structure for studying the interplay between principles dictating overlap dimensions and overall length of bipolar microtubule arrays.

\section{Acknowledgements}

We thank T. Miki, K. Kosetsu and G. Goshima (Nagoya University) for kindly sharing plasmids for $\alpha$-tubulin labelling. 
A

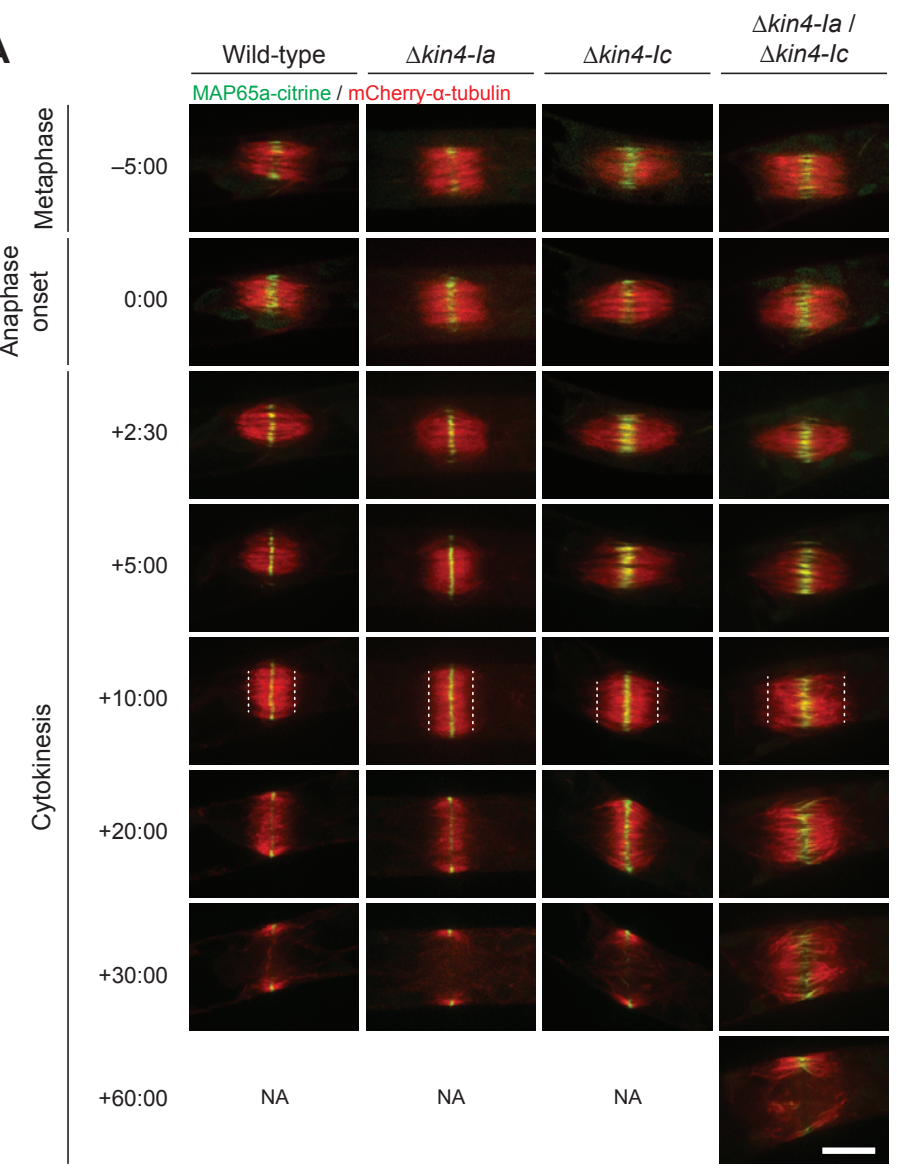

B

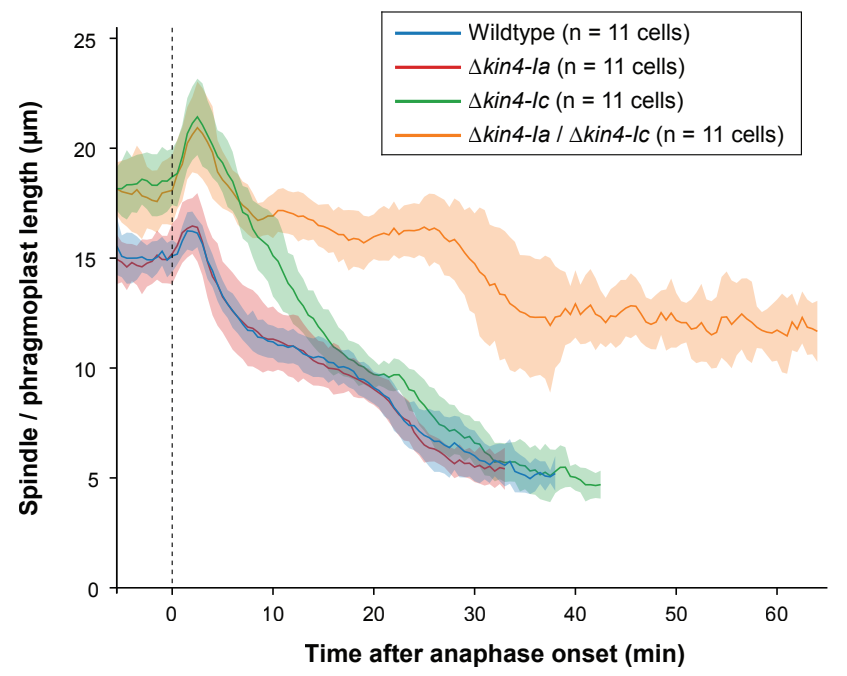

Figure 4 - Length of the division apparatus is set by kinesin-4 activity. (A) Image sequence of the entire cell division apparatus and microtubule overlaps visualized with mCherry-a-tubulin and MAP65a-citrine in wildtype cells and cells lacking Kin4-la and/or Kin4-Ic. The time with respect to anaphase onset $(t=0)$ is indicated in min:seconds on the left. Dashed lines highlight the varying pole-topole lengths of the division apparatus. Images are maximum z-projections of 3 confocal planes spaced $0.5 \mu \mathrm{m}$ apart and were rotated to keep the division plane vertically aligned. Scalebar, $10 \mu \mathrm{m}$. (B) Graph showing the evolution of the average, computationally determined spindle / phragmoplast length for the indicated amount of wild-type, $\Delta k i n 4-$ la, $\Delta k i n 4-I c$ and $\Delta k i n 4-l a ~ I$ $\Delta k$ in4-lc cells. The plots were trimmed at the end to only include averages obtained from at least half of the imaged cells. Shaded areas indicate standard deviation. 

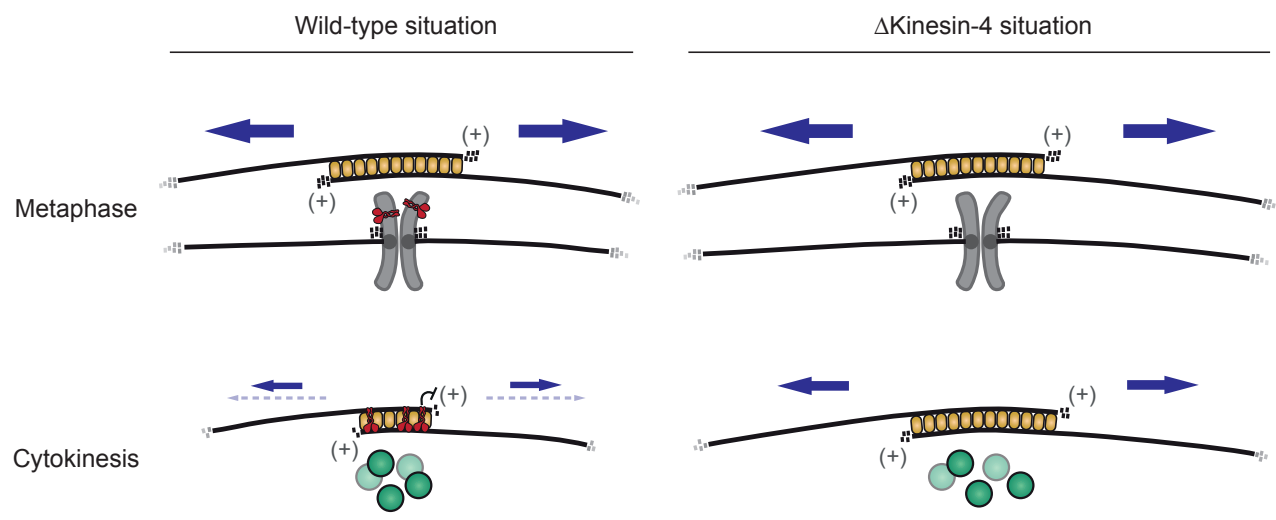

Figure 5 - Schematic depicting how interplay between microtubule growth, sliding and putatively minus end depolymerisation may determine spindle and phragmoplast length. In the wild-type situation (left hand side) in the metaphase spindle retrograde microtubule flux rates are high (blue arrows). To fuel this microtubule translocation, polymerisation rates must be high as well (black dashes). The apparatus during this stage in cell division retains a constant length, implying that depolymerisation at microtubule minus-ends occurs at an equal rate (gray dashes). As cytokinesis starts, recruitment of kinesin-4 (red) down-regulates growth at microtubule overlaps (yellow) and a concomitant slower pace of microtubule flux is observed. At the same time microtubule flux behaviour becomes heterogeneous among microtubules with a largely immobile population and one exhibiting sporadic fast retrograde movement (dashed arrow). In absence of kinesin-4 regulators of microtubule growth (right), flux rates are marginally enhanced in the metaphase spindle, potentially due to activities exerted from its chromosome-bound location at this stage (de Keijzer et al. 2017; Miki et al. 2014) and leading to a slightly longer division apparatus. Moreover, during cytokinesis, enhanced growth at microtubule overlaps enables a higher flux rate compared to wild-type. The phragmoplast length remains relatively constant throughout cytokinesis compared to the gradual shortening found in wild-type (Figure 4B). Possibly, in wild-type depolymerisation rates at minus ends exceed rates of plus end polymerization, whereas in the mutant situation this mismatch is partly compensated for by increased polymerization and flux.

\section{Materials and Methods}

\section{Plasmids and cloning procedures}

Plasmids used were generated using standard restriction-ligation cloning procedures and were verified by sequencing. The use of plasmids is summarized in table 2 and all primers are listed in table 1.

Table 1 - List of primers used in this study

\begin{tabular}{|c|c|c|c|}
\hline Primer & Use & Sequence $\left(5^{\prime}>3^{\prime}\right)$ & RE site \\
\hline JK225 & $\begin{array}{l}\text { Cloning of paGFP/pam- } \\
\text { Cherry fluorophore }\end{array}$ & ATTTGCGGCCGCGCAGAGATGGTGAGCAAGGGCGAGG & Notl \\
\hline JK226 & $\begin{array}{l}\text { Cloning of paGFP/pam- } \\
\text { Cherry fluorophore }\end{array}$ & $\begin{array}{l}\text { GACTAGTTGCTCCTGCTCCTGCTCCTGCTCCCTTGTA- } \\
\text { CAGCTCGTCCATGCC }\end{array}$ & Spel \\
\hline JK227 & $\begin{array}{l}\text { paGFP/pamCherry con- } \\
\text { struct sequencing }\end{array}$ & ATGCTCCAGACAGTACAGCTCC & - \\
\hline JK75 & $\begin{array}{l}\text { paGFP/pamCherry con- } \\
\text { struct sequencing }\end{array}$ & GACCACTACCAGCAGAACACC & - \\
\hline TM227 & $\begin{array}{l}\text { Cloning of mCherry } \\
\text { fluorophore }\end{array}$ & ATAAGCTTGATATCTTGGTGAGCAAGGGCGAGGAGCTG & HindIII \\
\hline
\end{tabular}


Table 1 - List of primers used in this study

\begin{tabular}{|c|c|c|c|}
\hline Primer & Use & Sequence (5'> 3') & RE site \\
\hline JK74 & $\begin{array}{l}\text { Cloning of mCherry } \\
\text { fluorophore }\end{array}$ & AGGAATTCTTACTTGTACAGCTCGTCCATGC & EcoRI \\
\hline JK129 & $\begin{array}{l}\text { Sequencing region } \\
\text { upstream of mCherry }\end{array}$ & CCCTTGGTCACCTTCAGCTTGG & - \\
\hline JK161 & $\begin{array}{l}\text { MAP65a locus geno- } \\
\text { typing }\end{array}$ & GGATGAGAACCGATTTGCGAGC & - \\
\hline JK162 & $\begin{array}{l}\text { MAP65a locus geno- } \\
\text { typing }\end{array}$ & GCGGTAAGAAAGTGGAGAGAAGC & - \\
\hline JK156 & EB1b locus genotyping & CATTTGTGGGTATGTGGGTTGG & - \\
\hline JK157 & EB1b locus genotyping & TGACAAAGGGAGAATAAAAGAGGTGG & - \\
\hline JK206 & $\begin{array}{l}\text { Kin4-la deletion geno- } \\
\text { typing }\end{array}$ & TTTGAGGTGTATCACCCAGTTTCG & - \\
\hline JK208 & $\begin{array}{l}\text { Kin4-la deletion geno- } \\
\text { typing }\end{array}$ & GCAGGAAGCACGAGCTAAGACC & - \\
\hline JK221 & $\begin{array}{l}\text { Kin4-la gene specific } \\
\text { primer - begin }\end{array}$ & GTTGAGACGCTGAAGGACAGTGC & - \\
\hline JK222 & $\begin{array}{l}\text { Kin4-la gene specific } \\
\text { primer - begin }\end{array}$ & CCAGCCAAGTCAACAAGGTGAAGC & - \\
\hline JK223 & $\begin{array}{l}\text { Kin4-la gene specific } \\
\text { primer - end }\end{array}$ & GCAGAATTGGTTGGAACAGGAGC & - \\
\hline JK224 & $\begin{array}{l}\text { Kin4-la gene specific } \\
\text { primer - end }\end{array}$ & TCCACATCTCGCCTCTTCACAGC & - \\
\hline
\end{tabular}

\section{ppaGFP-tua-BsdR}

The plasmid pTM425 featuring a GFP- $\alpha$-tubulin coding sequence with an upstream EF1 $\alpha$ promoter and a blasticidin resistance cassette (T. Miki, K. Kosetsu and G. Goshima, unpublished) was separated into three fragments by digestion: The backbone and blasticidin resistance cassette using $K p n \mathrm{I}$ and $A s c \mathrm{I}$, the EF1a promoter region using $K p n \mathrm{I}$ and $N o t \mathrm{I}$ and the $\alpha$-tubulin coding sequence using SpeI and $A s c \mathrm{I}$. These three fragments were combined with the paGFP coding sequence (L64F, T65S and T203H with respect to eGFP; Patterson \& Lippincott-Schwartz 2002) amplified using primers JK225 and JK226 and digested with SpeI and NotI, and then used in a 4-way ligation to yield a paGFP- $\alpha$-tubulin expression construct.

\section{ppamCherry1-tua-BsdR}

The same cloning procedure as described for ppaGFP-tua-BsdR was followed except that PAmCherry1 (Subach et al. 2009), amplified from plasmid pPAmCherry1-C1 (Addgene no. 31929) using primers JK225 and JK226, was used instead of paGFP.

\section{MAP65a-mCherry-nptII}

First, the sequence encoding the mCherry fluorophore (Shaner et al. 2004) was amplified with primers TM227 and JK74 and introduced in place of the citrine fluorophore in pCTRN-nptII (Hiwatashi et al. 2008) using HindIII and EcoRI sites. Next, the mCherry coding sequence and the nptII resistance cassette were obtained from this plasmid and used to replace the mCherry coding sequence and blasticidin resistance cassette of MAP65a-mCherry-BsdR (de Keijzer et al. 2017) using HindIII and NotI sites. 
Table 2 - List of plasmids used in this study

\begin{tabular}{|c|c|c|}
\hline Plasmid & Usage & Source \\
\hline pTM425 & $\begin{array}{l}\text { EF1 } \alpha \text {-promoter driven expression of GFP- } \alpha \text {-tu- } \\
\text { bulin }\end{array}$ & $\begin{array}{l}\text { (T. Miki, K. Kosetsu and G. } \\
\text { Goshima, unpublished) }\end{array}$ \\
\hline EF1-paGFP-tua-BsdR & $\begin{array}{l}\text { EF1 } \alpha \text {-promoter driven expression of paG- } \\
\text { FP-a-tubulin }\end{array}$ & This study \\
\hline EF1-pamCherry-tua-BsdR & $\begin{array}{l}\text { EF1 } \alpha \text {-promoter driven expression of pamCher- } \\
\text { ry1- } \alpha \text {-tubulin }\end{array}$ & this study \\
\hline MAP65a-mCherry-nptll & C-terminal mCherry tagging of MAP65a & This study \\
\hline EB1b-mCherry & $\begin{array}{l}\text { C-terminal mCherry tagging of expressed } \\
\text { EB1-homologue EB1b }\end{array}$ & See chapter 5 \\
\hline Kin4-la-KO-NatR & $\begin{array}{l}\text { Knock-out of the entire Kin4-la reading frame; } \\
\text { Nourseothricin resistance }\end{array}$ & (de Keijzer et al. 2017) \\
\hline pTM426 & $\begin{array}{l}\text { EF1a-promoter driven expression of mCher- } \\
\text { ry-a-tubulin }\end{array}$ & $\begin{array}{l}\text { (T. Miki, K. Kosetsu and G. } \\
\text { Goshima, unpublished) }\end{array}$ \\
\hline
\end{tabular}

\section{P. patens growth conditions and transformation}

$P$. patens tissues were routinely grown on BCDAT plates under continuous light, unless stated otherwise. Plasmids were linearized and introduced into the $P$. patens genome by homologous recombination using PEG-mediated protoplast transformation (Nishiyama et al. 2000). Correct insertion events were identified by PCR. Characteristics of generated moss lines and their use throughout the study are summarized in table 3. For imaging, protonemal tissue was grown on BCD medium in glass bottom dishes.

Table 3 - List of strains used in this study

\begin{tabular}{|c|c|c|c|c|c|c|}
\hline Name & Experimental use & $\begin{array}{l}\text { Main } \\
\text { clone } \\
\text { used }\end{array}$ & $\begin{array}{l}\text { Background } \\
\text { strain }\end{array}$ & $\begin{array}{l}\text { Plasmid } \\
\text { transformed }\end{array}$ & Figure & Reference \\
\hline EF1-paGFP-tua & $\begin{array}{l}\text { Substep in generating strain with } \\
\text { labelled overlaps and photoacti- } \\
\text { vatable microtubules }\end{array}$ & $\# 219$ & WT & $\begin{array}{l}\text { EF1-paGFP- } \\
\text { tua-BsdR }\end{array}$ & S1A & This study \\
\hline $\begin{array}{l}\text { MAP65a-mCherry / } \\
\text { EF1-paGFP-tua }\end{array}$ & $\begin{array}{l}\text { Characterization of retrograde } \\
\text { microtubule flux behaviour during } \\
\text { cell division }\end{array}$ & \#215 & EF1-paGFP-tua & $\begin{array}{l}\text { MAP65a- } \\
\text { mCherry-nptll }\end{array}$ & $1,2, \mathrm{~S} 1$ & This study \\
\hline MAP65c-citrine & $\begin{array}{l}\text { Parent strain for generating over- } \\
\text { lap + EB1b - colocalization strain }\end{array}$ & $\# 5$ & WT & see reference & - & $\begin{array}{l}\text { (Kosetsu et } \\
\text { al. 2013) }\end{array}$ \\
\hline $\begin{array}{l}\text { MAP65c-citrine / } \\
\text { EB1b-mCherry }\end{array}$ & $\begin{array}{l}\text { Polymerisation visualization in } \\
\text { overlaps }\end{array}$ & \#100 & MAP65c-citrine & EB1b-mCherry & S2 & $\begin{array}{l}\text { This study; } \\
\text { Chapter } 5\end{array}$ \\
\hline$\Delta k i n 4-/ c /$ MAP65a-cit & $\begin{array}{l}\text { Parent strain for generating } \\
\text { Kin4-la/lc knock-out strains with } \\
\text { additional red markers }\end{array}$ & \#172 & MAP65a-citrine & see reference & - & $\begin{array}{l}\text { (de Keijzer } \\
\text { et al. 2017) }\end{array}$ \\
\hline $\begin{array}{l}\text { EB1b-mCherry / } \Delta k i n 4- \\
\text { Ic / MAP65a-cit }\end{array}$ & $\begin{array}{l}\text { Substep in generating Kin4-la/ } \\
\text { Ic knock-out strain with EB1b } \\
\text { marker }\end{array}$ & \#68 & 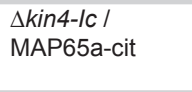 & EB1b-mCherry & $\mathrm{S} 2 \mathrm{~A}$ & This study \\
\hline $\begin{array}{l}\Delta \text { kin4-la / EB1b-mCher- } \\
\text { ry / } \Delta k i n 4-l c / \text { MAP65a- } \\
\text { cit }\end{array}$ & $\begin{array}{l}\text { Measuring effect of Kin4-la/ } \\
\text { Ic removal on polymerisation } \\
\text { dynamics in overlaps }\end{array}$ & \#86 & $\begin{array}{l}\text { EB1b-mCherry } \\
\text { / } \Delta \text { kin4-Ic / } \\
\text { MAP65a-cit }\end{array}$ & $\begin{array}{l}\text { Kin4-la-KO- } \\
\text { NatR }\end{array}$ & S2B-C & This study \\
\hline MAP65a-citrine & $\begin{array}{l}\text { Parent strain for generating } \\
\text { MAP65a-labelled (pa)mCher- } \\
\text { ry-tua expressing control strain }\end{array}$ & \#2 & WT & see reference & - & $\begin{array}{l}\text { (Kosetsu et } \\
\text { al. 2013) }\end{array}$ \\
\hline $\begin{array}{l}\text { pamCherry-tua / } \\
\text { MAP65a-cit }\end{array}$ & $\begin{array}{l}\text { Control strain with photo-activata- } \\
\text { ble microtubules for evaluation of } \\
\text { kinesin- } 4 \text { influence }\end{array}$ & \#34 & MAP65a-citrine & $\begin{array}{l}\text { EF1-pamCher- } \\
\text { ry-tua-BsdR }\end{array}$ & $\begin{array}{l}3 \\
\text { S3A-B }\end{array}$ & This study \\
\hline $\begin{array}{l}\text { pamCherry-tua / } \Delta k i n 4- \\
\text { Ic / MAP65a-cit }\end{array}$ & $\begin{array}{l}\text { Parent strain for generating } \\
\text { Kin4-la/lc knock-out strain with } \\
\text { photo-activatable microtubules }\end{array}$ & \#205 & $\begin{array}{l}\Delta k i n 4-I c / \\
\text { MAP65a-cit }\end{array}$ & $\begin{array}{l}\text { EF1-pamCher- } \\
\text { ry-tua-BsdR }\end{array}$ & - & This study \\
\hline
\end{tabular}


Table 3 - List of strains used in this study

\begin{tabular}{|c|c|c|c|c|c|c|}
\hline Name & Experimental use & $\begin{array}{l}\text { Main } \\
\text { clone } \\
\text { used }\end{array}$ & $\begin{array}{l}\text { Background } \\
\text { strain }\end{array}$ & $\begin{array}{l}\text { Plasmid } \\
\text { transformed }\end{array}$ & Figure & Reference \\
\hline $\begin{array}{l}\text { Dkin4-la / pamCher- } \\
\text { ry-tua / } \Delta \text { kin4-Ic / } \\
\text { MAP65a-cit }\end{array}$ & $\begin{array}{l}\text { Measuring effect of Kin4-la/lc re- } \\
\text { moval on retrograde microtubule } \\
\text { flux behaviour }\end{array}$ & \#533 & $\begin{array}{l}\text { pamCherry-tua } \\
\text { / } \Delta k \text { kin4-Ic / } \\
\text { MAP65a-cit }\end{array}$ & $\begin{array}{l}\text { Kin4-la-KO- } \\
\text { NatR }\end{array}$ & $3, \mathrm{~S} 3 \mathrm{~B}$ & This study \\
\hline $\begin{array}{l}\text { mCherry-tua / MAP65a- } \\
\text { cit }\end{array}$ & $\begin{array}{l}\text { Characterization of wild-type ap- } \\
\text { pearance and pole-to-pole length } \\
\text { of division apparatus }\end{array}$ & \#426 & MAP65a-citrine & pTM426 & 4 & This study \\
\hline$\Delta$ kin4-la / MAP65a-cit & $\begin{array}{l}\text { Parent strain for generating Kin4- } \\
\text { la knock-out strain with labelled } \\
\text { microtubules }\end{array}$ & $\# 7$ & MAP65a-citrine & see reference & - & $\begin{array}{l}\text { (de Keijzer } \\
\text { et al. 2017) }\end{array}$ \\
\hline $\begin{array}{l}\text { mCherry-tua / } \Delta k i n 4-l a ~ / ~ \\
\text { MAP65a-cit }\end{array}$ & $\begin{array}{l}\text { Measuring effect of Kin4-la } \\
\text { removal on pole-to-pole length }\end{array}$ & \#14 & $\begin{array}{l}\Delta k i n 4-l a ~ / \\
\text { MAP65a-cit }\end{array}$ & pTM426 & $4, \mathrm{~S} 3 \mathrm{C}$ & $\begin{array}{l}\text { This study; } \\
\text { Chapter } 5\end{array}$ \\
\hline $\begin{array}{l}\text { mCherry-tua / } \Delta k \text { in4-lc / } \\
\text { MAP65a-cit }\end{array}$ & $\begin{array}{l}\text { Measuring effect of Kin4-Ic } \\
\text { removal on pole-to-pole length; } \\
\text { Generation of double KO strain }\end{array}$ & \#474 & 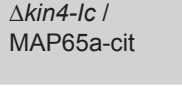 & pTM426 & 4 & This study \\
\hline $\begin{array}{l}\Delta \text { kin4-la / mCherry-tua / } \\
\Delta \text { kin4-Ic / MAP65a-cit }\end{array}$ & $\begin{array}{l}\text { Measuring effect of Kin4-la/lc } \\
\text { removal on pole-to-pole length }\end{array}$ & \#81 & $\begin{array}{l}\text { mCherry-tua } \\
\text { / } \Delta k i n 4-l c / \\
\text { MAP65a-cit }\end{array}$ & $\begin{array}{l}\text { Kin4-la-KO- } \\
\text { NatR }\end{array}$ & 4 & This study \\
\hline
\end{tabular}

\section{Fluorescence microscopy}

In all fluorescence microscopy experiments interphase caulonemal tip cells were used unless stated otherwise. Imaging was performed on a spinning disk confocal microscope consisting of a Nikon Ti-eclipse body equipped with a Yokogawa CSU-X1 spinning disk head and 100x Plan Apo VC objective (NA 1.40). Image digitization was done with a Photometrics Evolve 512 EMCCD camera with a $1.2 \mathrm{x}$ post-magnification fitted in front of the camera. Typical exposures used were 500-800ms for citrine and paGFP probes and 800-1000ms for mCherry and pamCherry probes while employing a camera electron multiplication gain of 300. For imaging of citrine and paGFP an excitation wavelength of $491 \mathrm{~nm}$ in combination with a 527/60 bandpass emission filter was used and for imaging of mCherry and pamCherry $561 \mathrm{~nm}$ excitation light in combination with a 595/50 bandpass emission filter was used. All components were operated by MetaMorph software.

Localised photo-activation was accomplished using an integrated FRAP/PA head consisting of two galvano-scanner mirrors operated at $6.5 \mathrm{kHz}$ by iLas2 software (Roper scientific). To photo-activate paGFP, $405 \mathrm{~nm}$ laser light generated by a $60 \mathrm{~mW}$ Melles-Griot 56RCS001 diode laser operated at $\sim 17 \%$ of its maximum output was passed through a ND 0.6 filter, then scanned for 15 passes on to a pre-defined region of the imaging plane. PAmCherry1 was photo-activated similarly, except that $\sim 1 \%$ of the $405 \mathrm{~nm}$ laser output and 1000 scan passes were used (Higher activation intensities caused background photo-activation the area surrounding the region intended for activation). Four frames were captured prior to activation and a second photo-activation pulse was administered approximately 5 minutes after the first to verify that the initially activated microtubules had exhibited true turnover and were not merely removed.

To categorize different stages of cell division during photo-activation experiments the following criteria were used:

- Metaphase spindle: The mitotic spindle 2 to 4 minutes after breakdown of the nuclear envelope. At this stage chromosomes have aggregated in the equatorial plane (Nakaoka et al. 2012). Chromosome separation did not commence until at least $\sim 3$ minutes after 
photo-activation.

- Phragmoplast initiation: Approximately 2.5-3.5 minutes after anaphase onset as assessed by the sharp rise in MAP65 labelling intensity at overlaps (de Keijzer et al. 2017). At this moment chromatids have been fully separated and microtubule overlaps in the wild-type situation have almost completed shortening.

- Solid phragmoplast: Between 5 till 15 minutes after anaphase onset. Microtubules of the phragmoplast are homogeneously distributed along the forming cell plate as assessed by continuous labelling of the phragmoplast midplane by MAP65.

- Ring phragmoplast: The phragmoplast is in contact or in close proximity of the cell cortex and microtubule depolymerisation has taken place in phragmoplast centre visible through disappeared and/or diminished MAP65 signal at this location.

\section{Image analysis}

\section{General}

All image analysis was performed using the FIJI distribution of Image 1.51 and custom written Matlab (Mathworks) code. Time-lapse image data was rotated (bicubic interpolation) such that the division plane was oriented vertically with the growing tip of the cell located on the right hand side. The gradual tilting of the division plane common in caulonemal cells, was subsequently countered in each image using the ImageJ 'MultiStackReg' plugin (Rigid body type translation using the MAP65 channel as reference). Kymographs were produced with Image J plugin 'MultipleKymograph'.

\section{Bulk flux rate determination}

For analysis of overall (bulk) flux behaviour a 35 pixels wide line segment perpendicular to the midplane was used to generate an average kymograph (Figure 1B). Activated zones on both sites of the midzone were analysed independently. The centre of mass of an activated zone was determined in the first 8 frames after photo-activation. For this only pixels above an intensity threshold were used. This threshold was defined as the average background pixel intensity measured in an area immediately adjoining the activated area with 8 (for paGFP) or 3 (for pamCherry) times the intensity standard deviation in that area added. Subsequently, simple linear regression on the calculated centres of mass was used to obtain bulk flux rates.

\section{paGFP and pamCherry bleaching assays}

A region of the sparse interphase microtubule network of subapical cells was photo-activated and segments of isolated microtubules that remained visible for the duration of imaging were selected using the polygon selection tool using spline interpolation in FIJI. The mean fluorescence intensity in this area was recorded after which measured fluorescence intensities were first baselined to 0 using the 4 -frame average intensity before photo-activation and then normalized to 1 using the 4 -frame average intensity after photo-activation.

\section{Spindle/phragmoplast length determination}

To obtain a computational estimate of the length of the division apparatus an image segmentation procedure was used to isolate it from the background followed by morphological operations on the resulting image mask (Figure S3C). First, the individual frames of rotated movies cropped to $240 * 180$ pixels were thresholded using the maximum entropy method employing 500 bins in the intensity histogram (Kapur et al. 1985). The obtained thresholds were smoothed over time with robust local regression smoothing using a second degree polynomial 
and a span width of $30 \%$. The temporally smoothed thresholds were then used to calculate a threshold mask for each image. Objects smaller than 20 pixels were removed from this mask and holes were filled in. The mask was then successively eroded and dilated with a disk-shaped structuring element with a radius of 1 pixel. Holes inadvertently generated by this procedure were filled in again to yield a contiguous object mask for each image. The average length of the $9 \%$ longest horizontal rows of pixels present in the image mask (with a minimum of 5 pixel rows) was then determined and used as pole-to-pole length approximation of the cell division apparatus. The generated masks and areas used for length calculation were visually inspected to verify consistency and robustness of the procedure.

\section{Statistical analysis}

For statistical validation, data were first tested for normality using the Shapiro-Wilk test $(\alpha=0.05)$ verified by visual inspection of Q-Q plots. When normality was assumed, data were evaluated using either an unpaired t-test ( 2 samples) or a one-way ANOVA analysis ( $>2$ samples). For data where a normal distribution was not assumed, either a Mann-Whitney U test (2 samples) or Kruskal-Wallis test with pairwise comparisons ( $>2$ samples) was employed. For all statistical analysis IBM SPSS Statistics version 22 or 23 was used. 


\section{Supplemental figures}

A
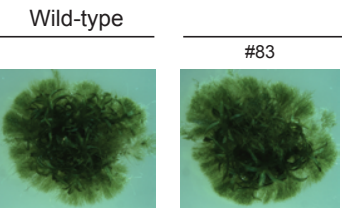

paGFP- $\alpha$-tubulin
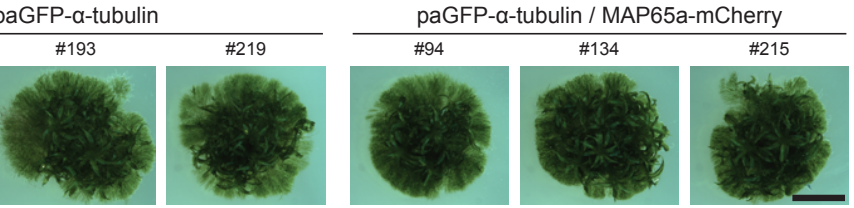

B
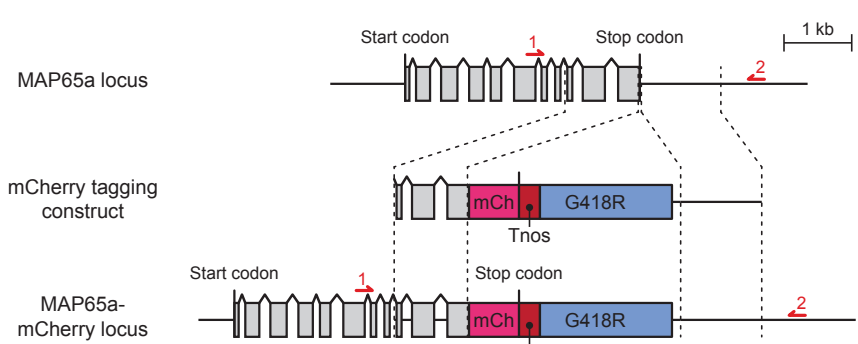

$1 \mathrm{~kb}$

MAP65a locus | 1 + 2: 3078bp MAP65a-mCherry locus | $1+2$ : 6199bp

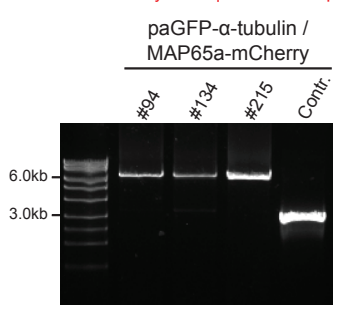

C
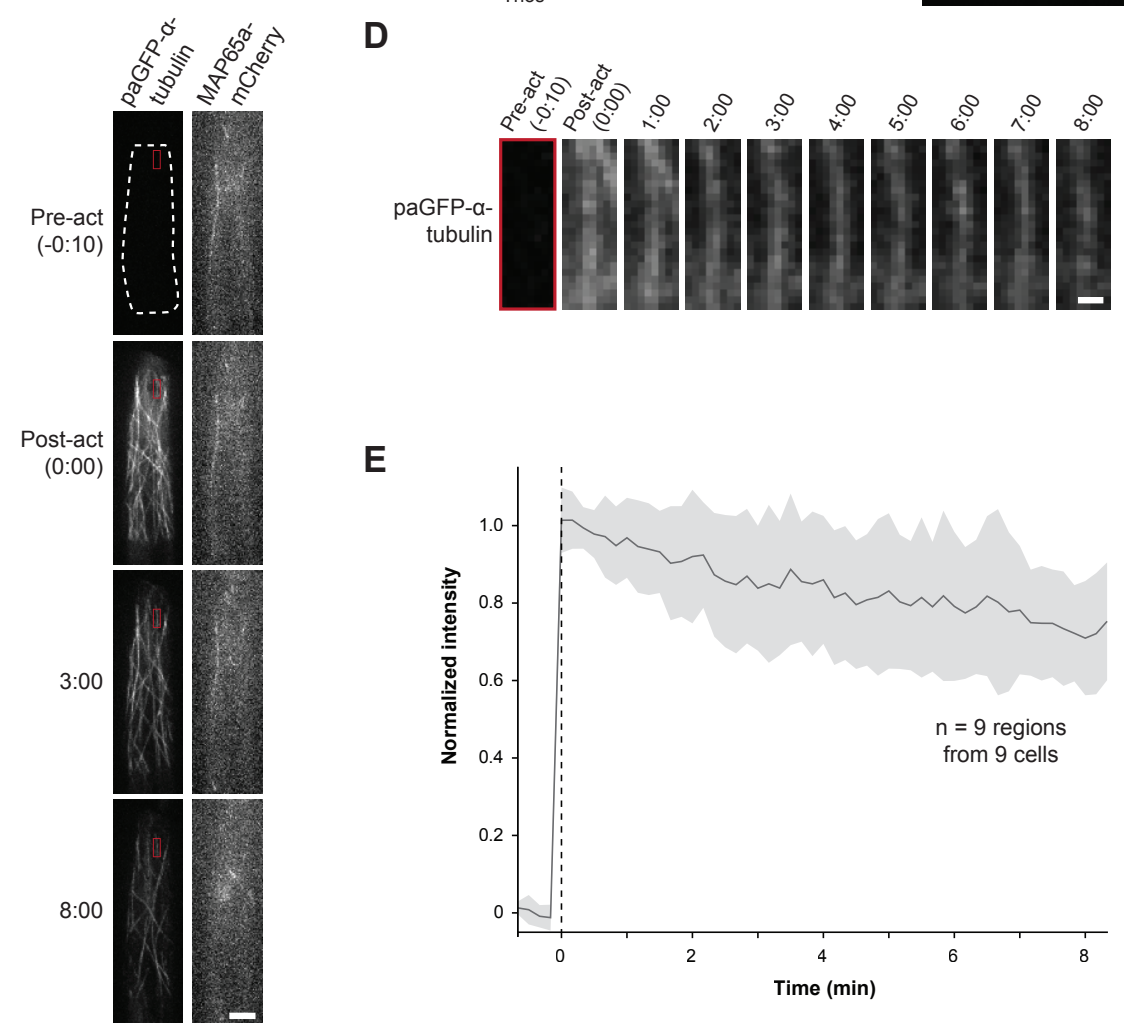

E

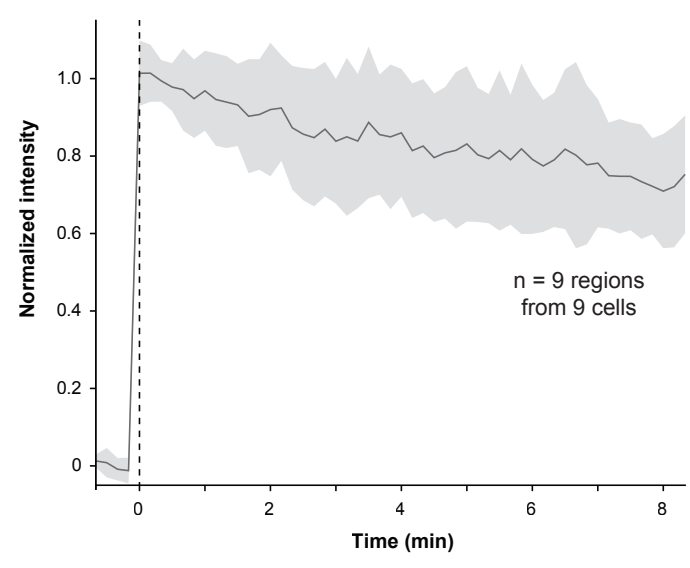

Figure S1 - Construction, confirmation and validation of a paGFP- $\alpha$-tubulin and MAP65a-mCherry expressing line. (A) Colony appearance of moss lines with an introduced paGFP-a-tubulin expression construct in a wild-type genetic background and lines with an additionally introduced MAP65a-mCherry marker. For each moss line three independent transformants are shown. Introduction of the expression constructs did not lead to altered colony size or morphology which would indicate growth defects. Plants were photographed after 4 weeks of growth on BCDAT agar plates. Scale bar, $5 \mathrm{~mm}$. (B) Schematic representation of the genomic locus containing the 
MAP65a encoding gene with its intron-exon structure (gray boxes) and the construct used for C-terminal mCherry tagging via homologous recombination (dashed lines). In MAP65a-labelled lines, the original stop codon is replaced with a fragment containing the mCherry encoding sequence (pink box), the nopaline synthase terminator (red box) and a cassette conferring G418 resistance (blue box). Red arrows denote primer binding sites used for confirmation of the obtained lines by PCR. The product obtained after PCR reaction and its predicted size are given on the right. The numbers of three independently obtained transformants are given above the gel images. (C) Photo-activation of a large area of the interphase microtubule network in a non-apical cell expressing MAP65a-mCherry and paGFP- $\alpha$-tubulin to assess bleaching characteristics of paGFP. The area indicated by the dashed line was irradiated by $405 \mathrm{~nm}$ wavelength light after which signal decay of labelled microtubules was monitored. Time is indicated in min:seconds. A microtubule segment visible during the entire observation time is highlighted by the red box and is shown in detail in $D$. Pictures are a maximum z-projection of 3 planes spaced $0.5 \mu \mathrm{m}$ apart. A constant intensity display range is used. Scalebar, $5 \mu \mathrm{m}$. (D) Zoomed view of the microtubule segment highlighted in C. Time after photo-activation is indicated in min:seconds. A constant intensity display range is used. Scalebar, $0.5 \mu \mathrm{m}$. (E) Average, normalized photo-bleaching profile of activated paGFP- $\alpha$-tubulin obtained from immobile microtubule segments in view for the entire observation time (as illustrated in $D$ ). The indicated number of regions were used. The shaded area indicates standard deviation. Measured fluorescence intensities were normalized both to 0 using the 4-frame average intensity before photo-activation and to 1 using the 4-frame average intensity after photo-activation. 
A
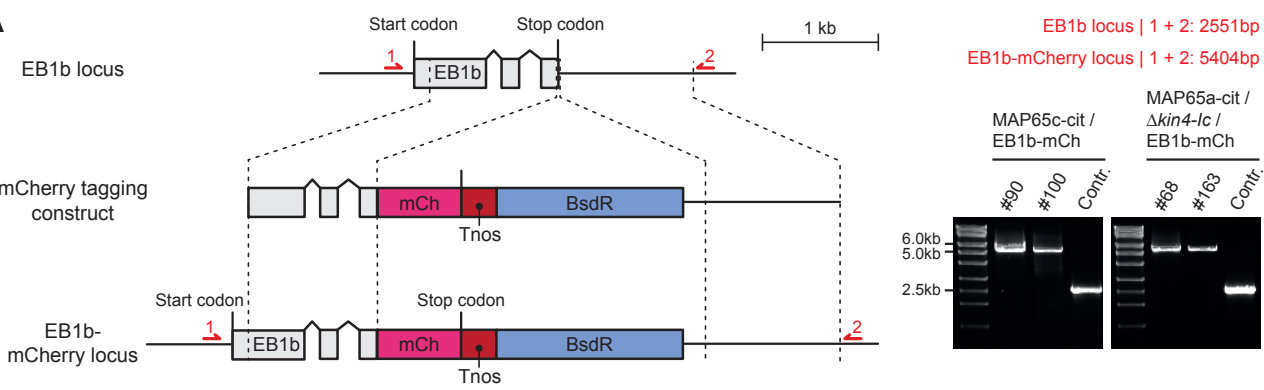

B

C

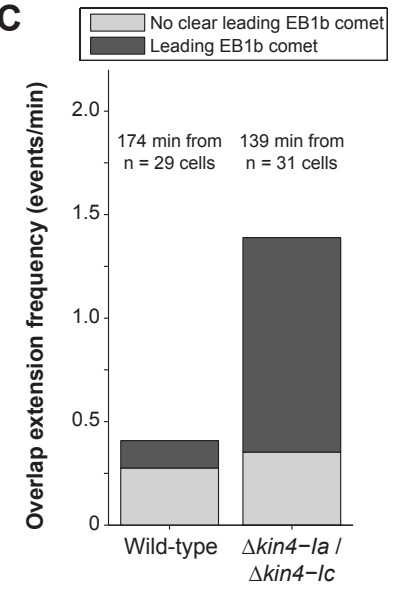

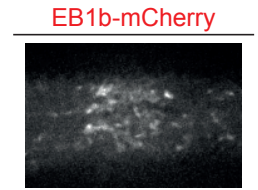

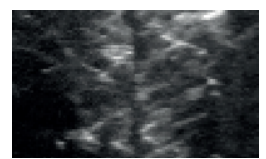

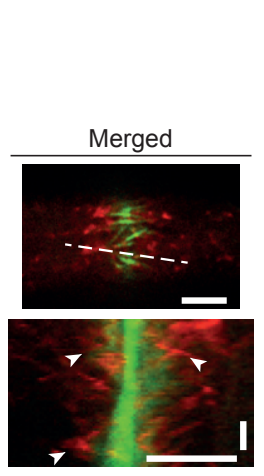

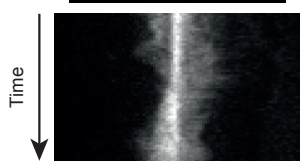

Figure S2 - Kinesin-4 prevents phragmoplast overlap elongation driven by microtubule polymerisation. (A) Schematic representation of the genomic locus containing the EB1b encoding gene with its intron-exon structure (gray boxes) and the construct used for C-terminal mCherry tagging via homologous recombination (dashed lines). In EB1b-labelled lines, the original stop codon is replaced with a fragment containing the mCherry encoding sequence (pink box), the nopaline synthase terminator (red box) and a cassette conferring blasticidin resistance (blue box). Red arrows denote primer binding sites used for confirmation of the obtained lines by PCR. The product obtained after PCR reaction and its predicted size are given on the right. The numbers of two independent transformants are given above the gel images. (B) Co-visualization of overlaps and growing microtubule plus ends in the phragmoplast of a $\Delta$ kin4-la / $\Delta k i n 4-I c$ cell expressing MAP65a-citrine and EB1b-mCherry. The dashed line indicates the region used for generation of the kymographs depicted below. Here, arrowheads mark overlap elongation foregone by an EB1b-marked polymerization event. Horizontal scalebars, $5 \mu \mathrm{m}$; vertical scalebar, $0.5 \mathrm{~min}$. (C) Graph representing the observed frequencies of overlap extension and fraction of EB1b-association in wild-type and kinesin-4 knock-out cells. Ring stage phragmoplasts were used for analysis and frequencies were obtained through inspection of kymographs as shown in B. Frequencies were derived from three serial optical planes only, and thus do not represent global rates. The total duration of the time-lapse data used is indicated. 
A

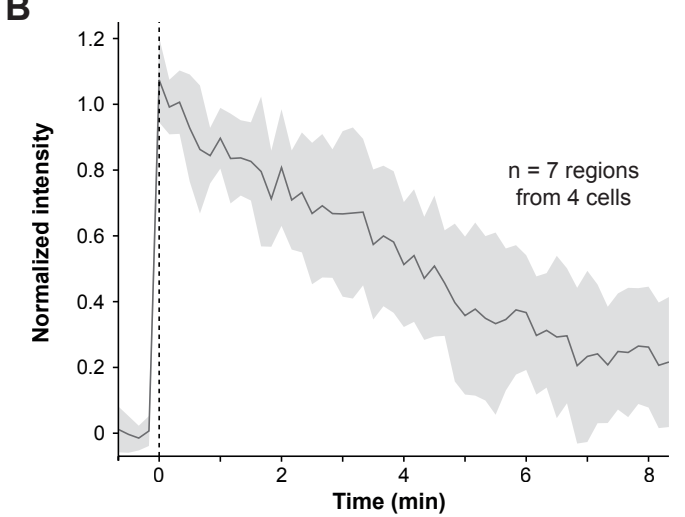

C
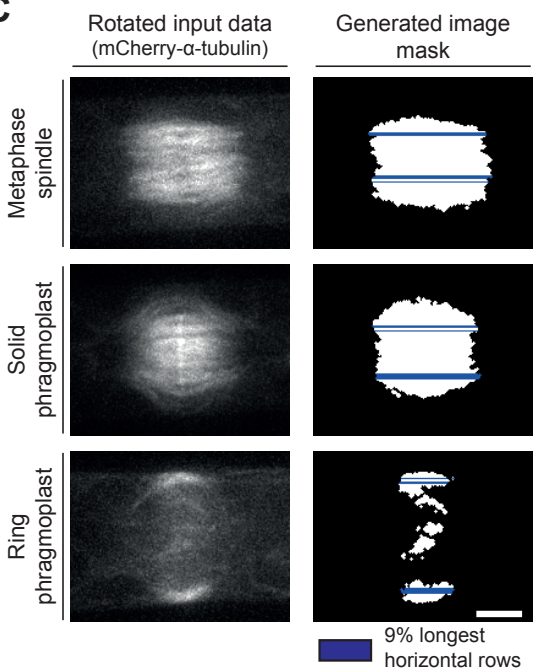

Figure S3 - Bleaching characterisation of pamCherry and automated determination of spindle and phragmoplast length. (A) Photoactivation experiment performed in the interphase microtubule network of a non-apical cell expressing pamCherry-a-tubulin and MAP65a-citrine (not shown) to assess bleaching characteristics of pamCherry. A small area containing a microtubule segment visible during the entire observation time is depicted. Time after photo-activation is indicated in min:seconds. Images are a maximum z-projection of 3 planes spaced $0.5 \mu \mathrm{m}$ apart. A constant intensity display range is used. Scalebar, $1 \mu \mathrm{m}$. (B) Average, normalized photo-bleaching profile of activated pamCherry- $\alpha$-tubulin obtained from immobile microtubule segments (as illustrated in A). The indicated number of activated microtubule regions were used, originating from both wildtype and $\Delta k i n 4-l a ~ / ~ \Delta k i n 4-I c$ genetic backgrounds. The shaded area indicates standard deviation. Measured fluorescence intensities were normalized both to 0 using the 4-frame average intensity before photo-activation and to 1 using the 4-frame average intensity after photo-activation. (C) Images of the cell division apparatus visualized with mCherry-a-tubulin and generated image masks at three different stages of division to illustrate the method used for computational approximation of spindle and phragmoplast length. In brief, image data rotated such that the midplane of the apparatus is aligned with the vertical axis, was thresholded using the maximum entropy method. Of the isolated objects the large features were retained and holes were filled to yield a continuous mask representing the division apparatus (right column). The average length of the $9 \%$ longest horizontal pixels rows (highlighted in blue) was then used as approximation of apparatus pole-to-pole length. This method maintained robust length approximation even as the segmented area started splitting in multiple areas during the ring phragmoplast stage (bottom row). Input images are a maximum z-projection of 3 planes spaced $0.5 \mu \mathrm{m}$ apart. Scalebar, $5 \mu \mathrm{m}$. 


\section{References}

Ando, D. et al., 2015. Cytoskeletal network morphology regulates intracellular transport dynamics. Biophysical Journal, 109(8), pp.1574-1582.

Asada, T., Kuriyama, R. \& Shibaoka, H., 1997. TKRP125, a kinesin-related protein involved in the centrosome-independent organization of the cytokinetic apparatus in tobacco BY-2 cells. Journal of cell science, 110 (2), pp.179-89.

Asada, T., Sonobe, S. \& Shibaoka, H., 1991. Microtubule translocation in the cytokinetic apparatus of cultured tobacco cells. Nature, 350, pp.238-241.

Bannigan, A. et al., 2007. A conserved role for kinesin-5 in plant mitosis. Journal of Cell Science, 120(16), pp.2819-2827.

Bowne-Anderson, H., Hibbel, A. \& Howard, J., 2015. Regulation of microtubule growth and catastrophe: Unifying theory and experiment. Trends in Cell Biology, 25(12), pp.769-779.

Braun, M. et al., 2011. Adaptive braking by Ase1 prevents overlapping microtubules from sliding completely apart. Nature Cell Biology, 13(10), pp.1259-1264.

Buster, D.W., Zhang, D. \& Sharp, D.J., 2007. Poleward tubulin flux in spindles: Regulation and function in mitotic cells. Molecular Biology of the Cell, 18(8), pp.3094-3104.

Gadde, S. \& Heald, R., 2004. Mechanisms and molecules of the mitotic spindle. Current Biology, 14(18), pp. $797-805$.

Ganem, N.J., Upton, K. \& Compton, D.A., 2005. Efficient mitosis in human cells lacking poleward microtubule flux. Current Biology, 15(20), pp.1827-1832.

Gillmor, C.S. et al., 2016. A genetic screen for mutations affecting cell division in the Arabidopsis thaliana embryo identifies seven loci required for cytokinesis PLoS one, 11(1), e0146492.

Goshima, G. \& Scholey, J.M., 2010. Control of mitotic spindle length. Annual Review of Cell and Developmental Biology, 26(1), pp.21-57.

Hepler, P.K. \& Jackson, W.T., 1968. Microtubules and early stages of cell-plate formation in the endosperm of Haemanthus katherinae Baker. Journal of Cell Biology, 38(2), pp.437-446.

Hiwatashi, Y. et al., 2008. Kinesins are indispensable for interdigitation of phragmoplast microtubules in the moss Physcomitrella patens. The Plant Cell, 20(11), pp.3094-3106.

Hiwatashi, Y., Sato, Y. \& Doonan, J.H., 2014. Kinesins have a dual function in organizing microtubules during both tip growth and cytokinesis in Physcomitrella patens. The Plant Cell, 26(3), pp.1256-1266.

Ho, C.-M.K. et al., 2011. Interaction of antiparallel microtubules in the phragmoplast is mediated by the microtubule-associated protein MAP65-3 in Arabidopsis. The Plant Cell, 23(8), pp.2909-2923.

Hu, C.-K. et al., 2011. KIF4 regulates midzone length during cytokinesis. Current Biology, 21(10), pp.815824.

Kapur, J.N., Sahoo, P.K. \& Wong, A.K.C., 1985. A new method for gray-level picture thresholding using the entropy of the histogram. Computer Vision, Graphics, and Image Processing, 29(3), pp.273-285.

de Keijzer, J. et al., 2017. Shortening of microtubule overlap regions defines membrane delivery sites during plant cytokinesis. Current Biology, 27(4), pp.514-520.

Komis, G. et al., 2017. Katanin effects on dynamics of cortical microtubules and mitotic arrays in Arabidopsis thaliana revealed by advanced live-cell imaging. Frontiers in Plant Science, 8.

Kosetsu, K. et al., 2013. MICROTUBULE-ASSOCIATED PROTEIN65 is essential for maintenance of phragmoplast bipolarity and formation of the cell plate in Physcomitrella patens. The Plant Cell, 25(11), pp.4479-4492.

Lee, Y.-R.J. \& Liu, B., 2013. The rise and fall of the phragmoplast microtubule array. Current Opinion in Plant Biology, 16(6), pp.757-763.

Lee, Y.J., Giang, H.M. \& Liu, B., 2001. A novel plant kinesin-related protein specifically associates with the phragmoplast organelles. The Plant Cell, 13(11), pp.2427-2439.

Loughlin, R. et al., 2011. Katanin contributes to interspecies spindle length scaling in Xenopus. Cell, 147(6), pp.1397-1407.

Miki, T. et al., 2014. Endogenous localizome identifies 43 mitotic kinesins in a plant cell. Proceedings of the National Academy of Sciences of the United States of America, 111(11), pp.1053-1061.

Mitchison, T.J., 2005. Mechanism and function of poleward flux in Xenopus extract meiotic spindles. Philosophical Transactions of the Royal Society B: Biological Sciences, 360(1455), pp.623-629.

Murata, T. et al., 2013. Mechanism of microtubule array expansion in the cytokinetic phragmoplast. Nature Communications, 4, p.1967. 
Nakaoka, Y. et al., 2012. An inducible RNA interference system in Physcomitrella patens reveals a dominant role of augmin in phragmoplast microtubule generation. The Plant Cell, 24(4), pp.1478-1493.

Nishiyama, T. et al., 2000. Tagged mutagenesis and gene-trap in the moss, Physcomitrella patens by shuttle mutagenesis. DNA Research, 7(1), pp.9-17.

Nislow, C. et al., 1992. A plus-end-directed motor enzyme that moves antiparallel microtubules in vitro localizes to the interzone of mitotic spindles. Nature, 359, pp.543-547.

Otegui, M.S. et al., 2001. Three-dimensional analysis of syncytial-type cell plates during endosperm cellularization visualized by high resolution electron tomography. The Plant Cell, 13(9), pp.2033-2051.

Palevitz, B.A., 1993. Morphological plasticity of the mitotic apparatus in plants and its developmental consequences. The Plant Cell, 5(9), pp.1001-1009.

Panteris, E. et al., 2011. A role for katanin in plant cell division: Microtubule organization in dividing root cells of fra2 and lue1 Arabidopsis thaliana mutants. Cytoskeleton, 68(7), pp.401-413.

Patterson, G.H. \& Lippincott-Schwartz, J., 2002. A photoactivatable GFP for selective photolabeling of proteins and cells. Science, 297(5588), pp.1873-1877.

Petry, S., 2016. Mechanisms of mitotic spindle assembly. Annual Review of Biochemistry, 85(1), pp.659-683.

Reber, S.B. et al., 2013. XMAP215 activity sets spindle length by controlling the total mass of spindle microtubules. Nature Cell Biology, 15(9), pp.1116-1122.

de Ruijter, N.C.A. et al., 1997. Spatial limitations induce spindle tilting and result in oblique phragmoplasts in Vicia faba L. root tip cells, but do not result in oblique cell walls. Acta Botanica Neerlandica, 46(3), pp.279-290.

Sawin, K.E. \& Mitchison, T.J., 1991. Poleward microtubule flux in mitotic spindles assembled in vitro. Journal of Cell Biology, 112(5), pp.941-954.

Shaner, N.C. et al., 2004. Improved monomeric red, orange and yellow fluorescent proteins derived from Discosoma sp. red fluorescent protein. Nature Biotechnology, 22(12), pp.1567-1572.

Shirasu-Hiza, M. et al., 2004. Eg5 causes elongation of meiotic spindles when flux-associated microtubule depolymerization is blocked. Current Biology, 14(21), pp.1941-1945.

Subach, F. V et al., 2009. Photoactivatable mCherry for high-resolution two-color fluorescence microscopy. Nature Methods, 6(2), pp.153-159.

Subramanian, R. \& Kapoor, T.M., 2012. Building complexity: insights into self-organized assembly of microtubule-based architectures. Developmental Cell, 23(5), pp.874-885.

Uehara, R. et al., 2013. Aurora B and Kif2A control microtubule length for assembly of a functional central spindle during anaphase. Journal of Cell Biology, 202(4), pp.623-636.

Vantard, M. et al., 1990. Incorporation of Paramecium axonemal tubulin into higher plant cells reveals functional sites of microtubule assembly. Proceedings of the National Academy of Sciences of the United States of America, 87(22), pp.8825-8829.

Wandke, C. et al., 2012. Human chromokinesins promote chromosome congression and spindle microtubule dynamics during mitosis. Journal of Cell Biology, 198(5), pp.847-863.

Wang, H. et al., 2010. Coupling between microtubule sliding, plus-end growth and spindle length revealed by kinesin-8 depletion. Cytoskeleton, 67(11), pp.715-728.

Wang, H. et al., 2013. Patronin mediates a switch from kinesin-13-dependent poleward flux to anaphase b spindle elongation. Journal of Cell Biology, 203(1), pp.35-46.

Yang, G. et al., 2008. Regional variation of microtubule flux reveals microtubule organization in the metaphase meiotic spindle. Journal of Cell Biology, 182(4), pp.631-639. 


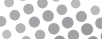

:8889.

:898898

-

$\because 89$

Chapter 5 - Kinesin4-la restricts growth

$\because$ of antiparallel microtubule overlaps

$\therefore$ to enhance array polarity and prevent

hyper-parallelization for responsive tip girowth guidance

Jeroen de Keijzer, Ruben van Spoordonk, Joanne E. van der Meer-Verweij, Marcel E. Janson and Tijs Ketelaar

Laboratory of Cell Biology, Wageningen University, Droevendaalsesteeg 1, 6708 PB Wageningen, The Netherlands

"Je gaat het pas zien als je het door hebt."

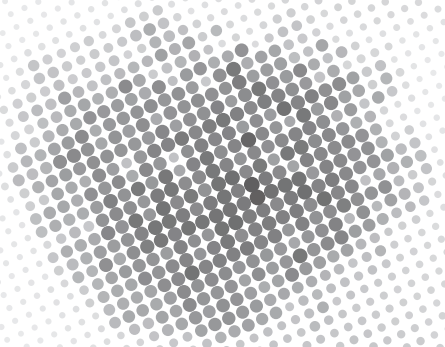




\section{Abstract}

Tip growing cells of amongst others plants and fungal hyphae secrete wall materials in a highly polarized fashion for fast and efficiently navigable colonization of the environment. The microtubule cytoskeleton has been implicated in generation of robustness and directional control over the growth process. The organizing principles of the polarized array in tip growing plant cells have remained particularly elusive as they lack a single defined microtubule nucleation site. Here we show that antiparallel encounters occur among the dispersedly nucleated microtubules in the polarized network of tip growing Physcomitrella patens caulonemal cells and identify a Kinesin- 4 molecule that is specifically recruited to these sites. Through direct visualization of microtubule plus end growth within antiparallel overlaps we demonstrate that this kinesin-4 inhibits polymerisation to confine overlap length. We then show that this confinement increases array polarization while simultaneously preventing hyper-parallelization of microtubules along the apical-basal axis. Loss of kinesin-4 activity results in a more stable growth axis and slower redirection of growth upon gravistimulation. Thus, the use of selective inhibition of microtubule growth at antiparallel overlaps constitutes a new organising principle for a polarized microtubule array which, during tip growth, guides expedient cell morphogenesis and provides responsiveness to environmental cues.

\section{Introduction}

Tip growth is a form of highly polarized cell growth used by a wide range of walled eukaryotes to rapidly colonize the immediate environment. Tip growth is encountered ubiquitously in the plant kingdom and occurs in select but diverse tissues (Domozych et al. 2013). The functioning of these tissues often intimately ties in with the typical long, cylindrical cell shape produced by tip growth, evident for example in the surface expansion of the root epidermis by root hairs in higher plants (Gilroy \& Jones 2000).

On the cellular level tip growth is realized by targeting of the secretory pathway to a defined growth zone. The major machinery functioning in the establishment and maintenance of the growth zone have been elucidated and generally include a dynamic actin network (reviewed in Ketelaar 2013) and Rho-related GTPase-integrated signalling through reactive oxygen species, calcium ions and phosphoinositides (reviewed in Cole \& Fowler 2006; Takeda et al. 2008; Kost 2008; Lee \& Yang 2008). However, how long-term robustness and guidance of the growth axis is achieved remains comparatively elusive.

The microtubule cytoskeleton was implicated in this process through pharmalogical experiments performed in a wide range of tip growing systems (root hairs (Bibikova et al. 1999; Ketelaar 2003), gymnosperm pollen (Anderhag et al. 2000) and moss protonemata (Doonan et al. 1988)). Furthermore, work on the microtubule regulator ARMADILLO-REPEAT KINESIN1 has shown that globally tuning microtubule growth dynamics is required for uniform root hair growth (Yang et al. 2007; Eng \& Wasteneys 2014). In recent work Hiwatashi et al. show that in tip growing moss protonemata a unipolarized microtubule array is formed with plus ends converging in an apical microtubule focus, the presence of which correlates with tip growth rate and directionality (Hiwatashi et al. 2014). Understanding microtubule array architecture and polarization may thus be key to learn about tip growth guidance.

Rather than using a fixed, centrosome-based organizing centre, the principles organising plant microtubule arrays are based on multiple types of microtubule-microtubule interactions (de 
Keijzer et al. 2014). These interactions and their ability to shape microtubule networks have been extensively studied in the quasi-2D cortical microtubule array of higher plants (Wasteneys \& Ambrose 2009). In this microtubule array it was demonstrated that lateral encounters between microtubules can result in bundling, a powerful principle to reinforce an existing microtubule orientation (Dixit \& Cyr 2004). The conserved class of MAP65 bundling proteins plays a prominent role in the bundling of microtubules of opposite polarity (Chan et al. 1999; Smertenko et al. 2004). In vitro reconstitution assays have shown that MAP65 proteins control the bundling probability of two colliding microtubules through dimerization (Gaillard et al. 2008; Tulin et al. 2012). Interestingly, this bundling activity generally does not alter the parameters of dynamic instability of the microtubules involved (Bieling et al. 2010; Shaw \& Lucas 2011). However, in bipolar mitotic microtubule arrays MAP65 and its yeast and animal orthologues recruit other microtubule growth regulating factors to modulate the length of anti-parallel overlaps (Bratman \& Chang 2007; Bieling et al. 2010; de Keijzer et al. 2017). In this study we set out to investigate if the polarity-sensitive nature of the bundler MAP65 and its potential to recruit other microtubule growth regulators are used to modulate the topology of a unipolar array using the tip growing cells of the moss Physcomitrella patens.

First we identify one kinesin-4 type molecular motor that is selectively recruited to antiparallel microtubule bundles in interphase. We then demonstrate that this molecule selectively inhibits microtubule growth within these overlaps to aid in maintenance of overall network polarity and that the lack of this activity results in a rigid, less adaptable growth axis. Thus, overlapbased growth inhibition constitutes a new organising principle for reinforcing the polarity of a unipolar microtubule array vital for the correct guidance of growth processes.

\section{Results and discussion}

The microtubule plus end growth direction in the microtubule network of tip growing moss caulonemal apical cells is highly polarized towards the apex (Hiwatashi et al. 2014). While such polarity could theoretically be solely maintained through a bias in nucleation direction of new microtubules, the divergent angles of branched microtubule nucleation reported for protonemal cells (Nakaoka et al. 2015) suggests that also inter-microtubule interactions assist in polarity establishment after nucleation. To investigate if antiparallel microtubule encounters could be one of these interactions we imaged the antiparallel microtubule overlap marker MAP65a-citrine (Kosetsu et al. 2013) together with the microtubule network in tip growing caulonemal apical cells. This revealed the presence of distinct, bar-shaped areas of MAP65a-signal throughout the microtubule network (Figure 1A). These areas of overlap occurred both among the microtubules residing in the cortical cytoplasm and among these in the organelle-rich endoplasm (Figure S1A). This finding implies that throughout the cell volume the microtubule network contains areas where microtubules of opposing polarity are aligned. In the light of MAP65-family proteins being able to recruit specific modulators of microtubule dynamics (Bratman \& Chang 2007; Bieling et al. 2010; Duellberg et al. 2013; de Keijzer et al. 2014) the observed overlaps could serve as a nexus for the selective alteration of microtubule behaviour.

Since in a previous study we found that kinesin- 4 motors can fulfil such a role on microtubule overlaps present in the phragmoplast array of $P$. patens we focussed on this subfamily of molecular motors (de Keijzer et al. 2017). Using a collection of strains expressing citrine-tagged versions of all 8 kinesin-4 motors encoded in the P. patens genome (Shen et al. 2012; Miki et al. 2014), we found that only labelling of Kinesin4-Ia (Kin4-Ia) produced striped, MAP65-like patterns 
A
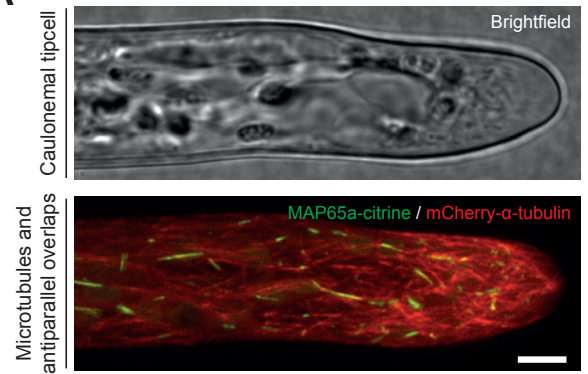

D

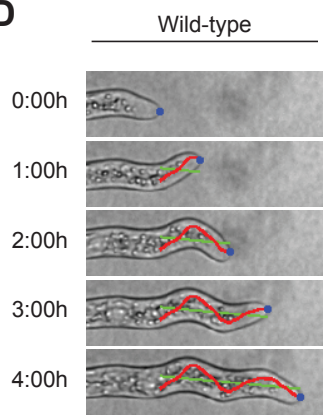

F
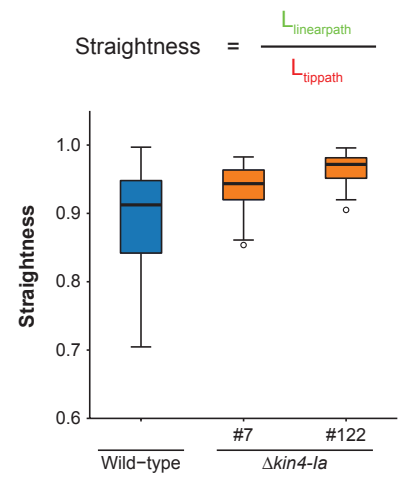

\begin{tabular}{lcc}
$\begin{array}{l}\text { P-values of pairwise } \\
\text { comparisons }\end{array}$ & $\begin{array}{l}\text { Mean } \\
\text { Ranks }\end{array}$ & $\begin{array}{c}\text { Variance } \\
\text { of Ranks }\end{array}$ \\
\hline Wild-type / $\Delta k i n 4-l a ~ \# 7$ & 0.131 & $\mathbf{0 . 0 4 3}$ \\
\hline Wild-type / $\Delta k i n 4-l a \# 122$ & $\mathbf{0 . 0 0 0}$ & $\mathbf{0 . 0 2 1}$ \\
\hline$\Delta k i n 4-l a \# 7$ / $\Delta k i n 4-l a \# 122$ & $\mathbf{0 . 0 2 1}$ & 0.646 \\
\hline
\end{tabular}

G
B
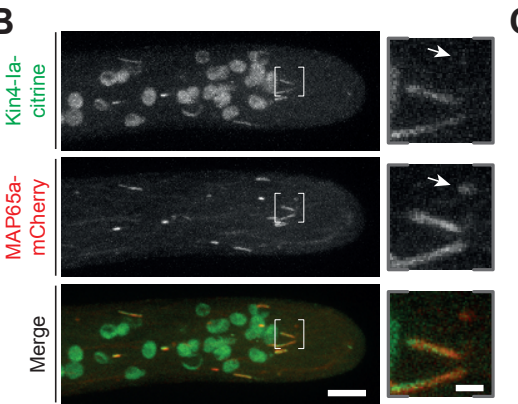

C \begin{tabular}{|l} 
Kin4-la \\
association \\
No Kin4-la \\
association \\
Uncertain \\
$n=598$ overlaps
\end{tabular} $n=598$ overlap

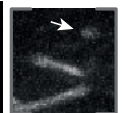

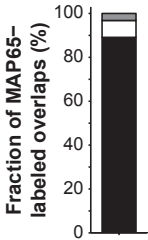
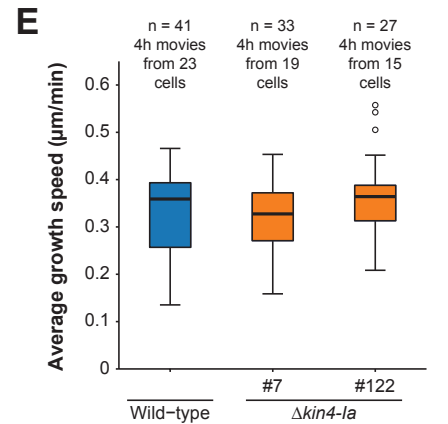

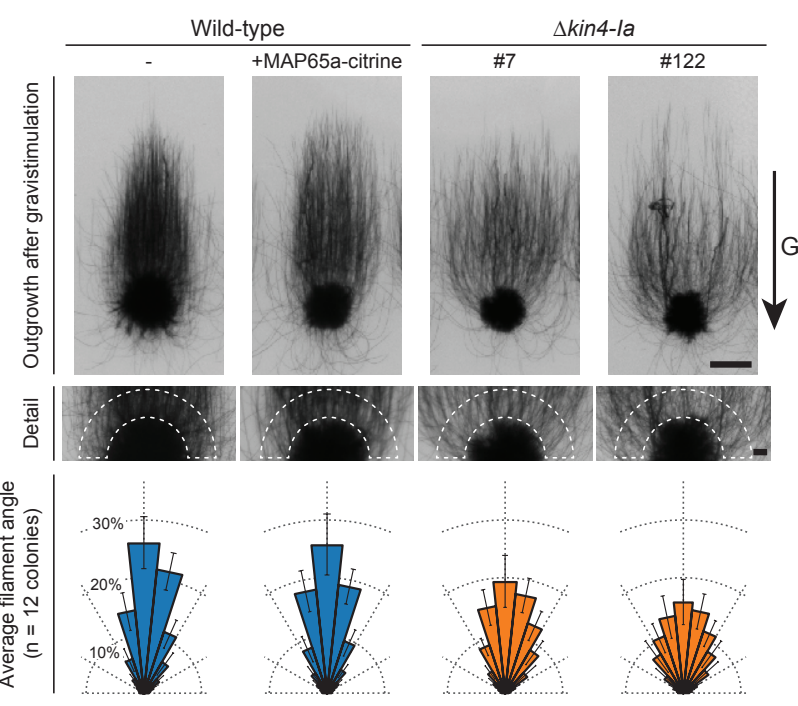

Figure 1 - In caulonemal apical cells Kin4-la localizes to antiparallel microtubule overlaps during interphase and loss of Kin4-la reduces the ability of caulonemal cells to alter their growth direction. (A) Microtubule network in a $P$. patens caulonemal tip cell and occurrence of antiparallel microtubule overlaps therein visualized in a cell expressing mCherry-a-tubulin and MAP65a-citrine. Fluorescence picture is a maximum z-projection of 20 planes spaced $0.4 \mu \mathrm{m}$ apart. Scalebar, $5 \mu \mathrm{m}$. (B) Interphase caulonema tip cell expressing both MAP65a-mCherry as a marker for antiparallel overlaps and Kin4-la labelled with citrine. An enlarged view of the bracketed area is shown on the right. Here, the arrow indicates an overlap without discernible Kin4-la-citrine association. The spherical objects also visible in the Kin4-la-citrine channel are autofluorescent chloroplasts. Images are a maximum z-projection of 20 planes spaced $0.4 \mu \mathrm{m}$ apart. The Kin4-la-citrine signal was acquired 
in caulonemal apical cells (Figure S1B). To confirm that the observed patterns corresponded to regions of antiparallel microtubule overlap a moss line expressing both Kin4-Ia-citrine and MAP65a-mCherry was constructed (Figure S2A). This revealed that indeed Kin4-Ia staining patterns co-localized with MAP65a-labelled regions of overlap (Figure 1B); 89\% of MAP65amCherry labelled overlaps showed concomitant Kin4-Ia localization (Figure 1C). Thus, besides having an established role at the phragmoplast midplane during late cytokinesis (de Keijzer et al. 2017), Kin4-Ia could have a role in organizing interphase microtubules for tip growth.

To establish if the presence of Kin4-Ia modulates tip growth behaviour we traced the growing tip of caulonemal cells over 4-hour periods in wild-type and two independently obtained Kin4-Ia knock-out lines (de Keijzer et al. 2017) (Figure 1D). The resulting trajectories were used to measure the speed of tip growth and the straightness of the path traversed by the tip relative to an idealized linear trajectory. The speed of tip growth was not significantly different among wild-type and the two $\Delta$ kint-Ia mutant strains (Figure 1E). This is in line with the absence of a phenotype at the level of colony size and appearance upon Kin4-Ia disruption (de Keijzer et al. 2017). However, calculation of a straightness indicator showed that, although the mean path straightness over a 4-hour period was similar to the wild-type for one mutant and slightly higher for the other, the variance decreased at least 4-fold in both mutants (Figure 1F). This highlights that upon losing Kin4-Ia function the growth axis of individual cells is more rigidly confined to a certain direction. To further address if the capability to steer the growth axis was compromised in $\Delta$ kin4-Ia mutants we used a gravitropic response assay. When transferred to the dark, caulonemal cells normally exhibit a strong, negative gravitropic growth response (Cove et al. 1978; Jenkins et al. 1986). Although in $\Delta k$ in4-Ia mutant lines the growth axis of caulonemal filaments ultimately aligned opposite to the direction of the gravity vector, compared to control lines alignment was delayed, indicating a hampered adaptation of tip growth direction (Figure $1 \mathrm{G})$. Taken together, these results show that Kin4-Ia disruption, without notably affecting the

before that of MAP65a-mCherry at each confocal plane. Scale bar in overview image, $5 \mu \mathrm{m}$; scale bar in the enlarged view, 1 Hm. (C) Bar graph representing the fraction of MAP65a-mCherry-labelled overlaps with an associated Kin4-la-citrine signal. The uncertain category was assigned in case close proximity of an autofluorescent chloroplast hampered the assessment of co-localization. (D) Time sequences of growing caulonemal tip cells of wild-type and two $\Delta k i n 4-l a$ mutant lines observed with bright field microscopy. The path travelled by the cell tip (blue dot) is highlighted in red. An idealised linear path based on linear regression analysis of the red path is indicated in green. Scale bar, $10 \mu \mathrm{m}$. (E) Boxplots of average tip growth speeds derived from 4 hour growth periods for wild-type and two $\Delta$ kin4-la lines. The whiskers indicate minimum / maximum observed values or 1.5 times the interquartile range when outliers (circles) were present. A one-way ANOVA analysis did not reveal significant differences among distributions $(P>0.05)$. ( $F)$ Definition of a straightness parameter wherein colours correspond to paths plotted in $\mathrm{D}$. Boxplots of the straightness parameter for paths travelled by cell tips of wild-type and two $\Delta$ kin 4 -la lines. The same 4 hour growth periods were analysed as indicated in $\mathrm{B}$. The outcome of a Kruskal-Wallis pairwise comparison test on the means as well as the outcome of pairwise Levene's tests for equal variance of the ranked dataset are represented in the table below. Significant differences $(P<0.05)$ are highlighted in bold. The whiskers indicate minimum / maximum observed values or 1.5 times the interquartile range when outliers (circles) were present. (G) Colony outgrowth photographed 5 weeks after transferring plates pre-grown in the light in a horizontal position to the dark in an upright position for two wild-type backgrounds (with and without the MAP65a-citrine marker present) and two $\Delta$ kin4-la mutant lines. Detailed pictures show that caulonema radiate out of the colony in a less polarized manner upon loss of Kin4-la. Here, the dashed lines indicate the $2 \mathrm{~mm}$ circumferential area at the top of the colony used to determine filament angles with OrientationJ. The circular histograms ('rose plots') below depict the average abundance of determined filament angles divided in $12^{\circ}$ bins. Error bars represent standard deviation. Scale bar in the overview images, $5 \mathrm{~mm}$; scale bar in the detail images, $1 \mathrm{~mm}$. 
rate of the growth process itself, has a major effect on the ability of a caulonemal cell to steer its growth axis.

Having established overlap association and a role in growth axis control for Kin4-Ia we next examined the dynamic behaviour of this motor more closely. In vitro assays with Xenopus MAP65homolog PRC1 and its associated kinesin-4 Xklp1 have elaborated how these two components can control overlap length (Bieling et al. 2010). After autonomous recognition of antiparallel overlaps by PRC1 it recruits Xklp1, which then, owing to its motility, inhibits growth at plus ends. Taking advantage of the high accessibility of caulonemal apical cells for live cell imaging we set out to investigate if similar mechanisms are involved in overlap formation in moss, using cells with both MAP65a and Kin4-Ia labelled. Imaging was performed in the cortical cytoplasm of the cell as here an extensive microtubule network is present and the potential of a local bias in microtubule orientations introduced through spatial hindrance by large organelles is minimal (Figure S1A). Observation of 70 overlap formation events ( $\mathrm{n}=15$ cells) revealed that Kin4-Iacitrine appeared on overlaps slightly later than its decoration by MAP65a-mCherry (always at least one $\sim 3 \mathrm{~s}$ interval later) and that after this initial delay both signals remained associated until overlap disappearance (Figure 2A). This observation is reminiscent of the kinesin-4 recruitment strategy described in vitro (Bieling et al. 2010) and could explain the small fraction of overlaps without apparent Kin4-Ia association we observed earlier (Figure 1C).

A

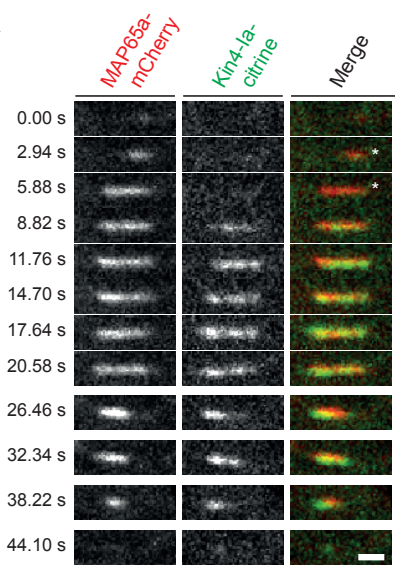

B

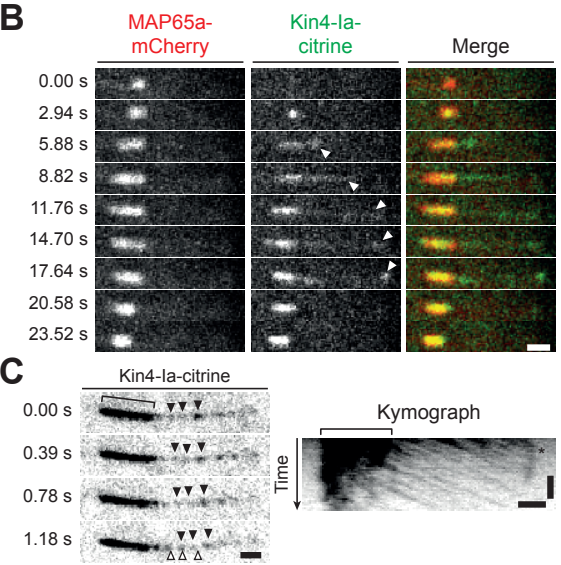

D

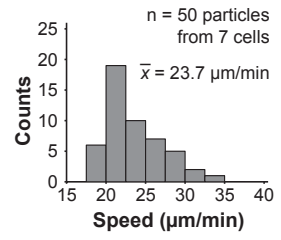

E

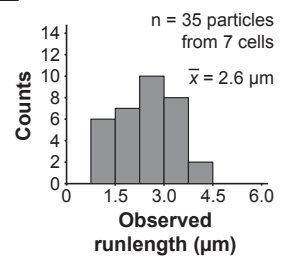

Figure 2 - Kin4-la is recruited to antiparallel overlaps and exhibits long-range motility. (A) Recruitment of Kin4-la to overlaps visualized by time-lapse imaging of a cell expressing Kin4-lacitrine and MAP65a-mCherry. Initially the expanding overlap is devoid of Kin4-la-citrine (indicated with an asterisk), which, after its later arrival, remains associated with the overlap until overlap disappearance. Images were obtained in a single optical plane from the cortical area. The Kin4-la-citrine signal was acquired before that of MAP65a-mCherry at each time point. Scalebar, $1 \mu \mathrm{m}$. (B) Snapshots showing asymmetric recruitment dynamics of Kin4-la onto microtubules adjacent to a nascent overlap. The arrowheads indicate a spur of Kin4-la-citrine fluorescence traveling in opposite direction of overlap elongation. Scalebar, $1 \mu \mathrm{m}$. (C) Time sequence of an overlap taken at high time resolution. Arrowheads point to examples of particles moving away from the overlap region (indicated with a bracket). The open arrowheads indicate previous particle positions at $t=0$. A kymograph representation depicted on the right shows that individual particles exhibit sustained motility away from the overlap until reaching a stationary zone of Kin4-la-citrine signal (marked with an asterisk). Horizontal scalebars, $1 \mu \mathrm{m}$; vertical scalebar, $5 \mathrm{~s}$. (D) Histogram showing the distribution of velocities for particles moving away from an overlap region. (E) Histogram showing the observed runlengths of particles. 
Interestingly, in $\sim 61 \%$ of the observed cases the Kin4-Ia-citrine signal also created a dim spur emanating from one side of the overlap (Figure 2B). Since we anticipate that in a diffusely nucleated microtubule network lateral encounters between a growing plus end and the lattice of an existing microtubule account for most overlap formation events, these spurs could correspond to the motor exploring a microtubule segment extending beyond the zone of overlap through plus end directed motility (Bieling et al. 2010). Indeed, imaging at high temporal resolution showed that these spurs consisted of particles moving in a highly linear fashion (Figure 2C). The average speed of these particles $(23.7 \mu \mathrm{m} / \mathrm{min})$ far exceeded typical microtubule polymerisation rates (Oda et al. 2009; Hiwatashi et al. 2014) and observed runlenghts were $2.6 \mu \mathrm{m}$ on average (Figure 2D-E). This suggests that Kin4-Ia could use its mobility to processively explore microtubules and reach growing plus-ends much akin to the behaviour of Xklp1 within and outside overlap zones described in vitro (Bieling et al. 2010). However, in caulonemal cells we could not directly observe motion within overlaps, probably owing to the high particle density and/or the presence of two microtubules of opposite polarity enabling frequent directional switching (Bieling et al. 2010). We conclude that the moss MAP65-Kin4-Ia system shares several relevant properties with the module described by Bieling et al. (2010) and as such could fulfil a similar role in controlling the formation and length of microtubule overlaps.

To investigate if Kin4-Ia affects the dimensions of antiparallel overlaps in caulonemal tip cells we measured the length of MAP65a-citrine labelled overlaps in the two Kin4-Ia knock-out lines and compared it with wild-type (Figure 3A). In the wild-type situation a narrow overlap length distribution with an average length of $0.91 \mu \mathrm{m}$ was observed (Figure 3B). By comparison, the length distribution in knock-out lines tapered off more slowly resulting in more than doubled average overlap lengths (Figure 3B). In both genetic backgrounds overlap lengths appeared uncorrelated with their relative position along the cell's main axis, highlighting that Kin4-Ia uniformly influences overlaps within the sampled area (Figure S2B). Thus, Kin4-Ia is a major determinant for overlap length in interphase caulonemal cells. This distinct role of Kin4-Ia also allowed us to verify that the citrine-tagged version of Kin4-Ia exhibited normal biological function, since a wild-type-like overlap length distribution was observed in cells expressing the fusion protein (Figure S2C).

To understand how Kin4-Ia limits overlap length we followed the dynamics of overlap formation events in wild-type and $\Delta k i n 4$-Ia lines (Figure 3C). Since overlap length limitation by Xklp1 acts via inhibition of polymerisation of the constituent microtubules (Bringmann et al. 2004; Bieling et al. 2010) we determined the average rate of overlap extension by dividing the maximum attained length of an overlap by the time taken to reach this length (Figure 3C, schematic). Compared to wild-type this rate was significantly higher in both $\Delta$ kin4-Ia lines indicating that growth inhibition could underlie the limitation of overlap length (Figure 3D).

In order to assay Kin4-Ia's role on polymerisation within overlaps more directly we introduced a marker for growing plus ends by tagging endogenous moss EB1-homolog EB1b with mCherry in both a wild-type and $\Delta$ kin4-Ia genetic background (Figure S3A) (Hiwatashi et al. 2014). We then simultaneously imaged citrine labelled MAP65 and EB1b-mCherry and measured the time that EB1b-comets associated with a nascent overlap and their average speed during that time (Figure 4). We found that comet lifetime in overlaps increased almost two-fold on average in absence of Kin4-Ia, while the average comet velocity only mildly increased in the mutant situation (Figure 4A-B). These measurements therefore not only expose that Kin4-Ia-dependent overlap length limitation acts chiefly via polymerisation inhibition, but also that the underlying 
A

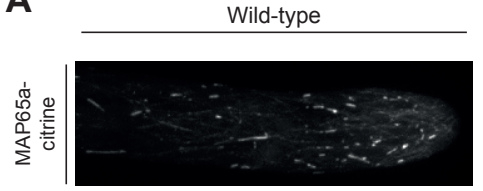

B

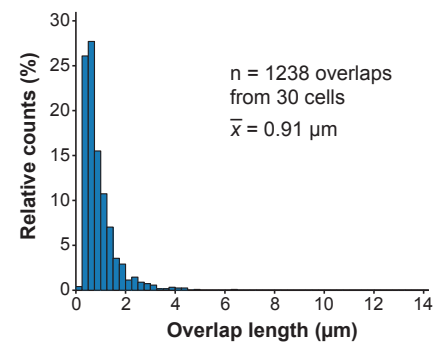

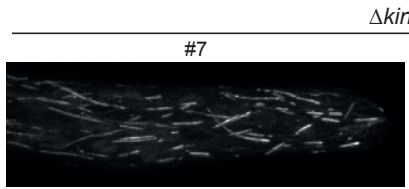

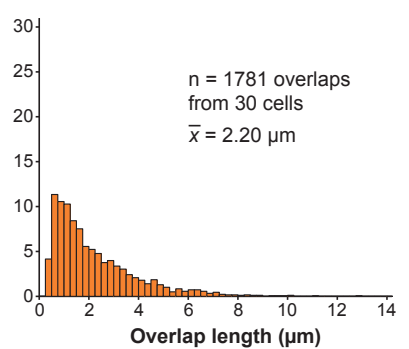

Overlap length $(\mu \mathrm{m})$
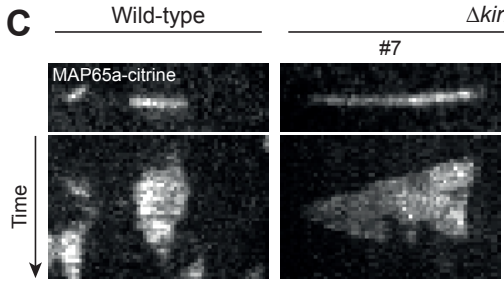

$\Delta$ kin4-la
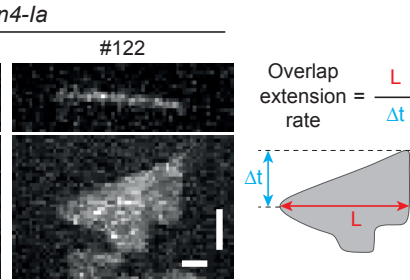

kin4-la
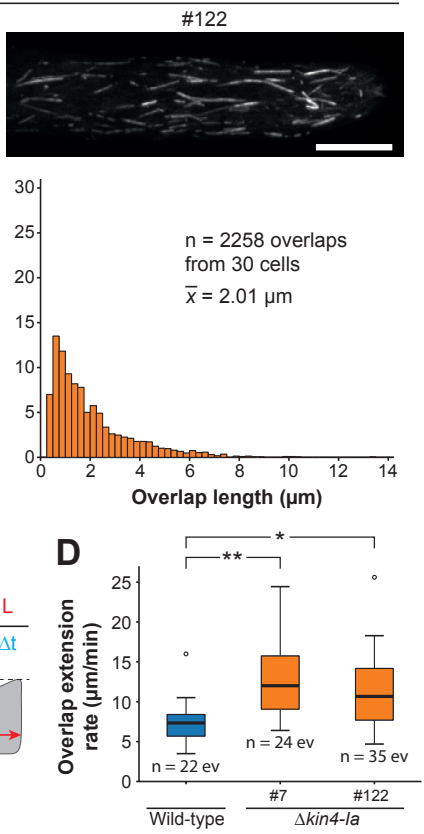

Figure 3 - Kin4-la limits overlap length by restricting the rate of overlap extension. (A) Visualization of overlaps by MAP65a-citrine in wild-type and $\Delta$ kin4-la cells. Two independent mutant strains are shown. Overlap regions appear longer in absence of Kin4-la. Horizontal scale bar, $10 \mu \mathrm{m}$. (B) Histograms showing the length distribution derived from the indicated amount of MAP65a-citrine labelled overlaps for wild-type and two $\Delta$ kin4-la mutant lines. All distributions were significantly different from each other in a Kruskal-Wallis test with pairwise comparisons $(P<0.001)$. (C) Kymographs and corresponding snapshots of microtubule overlap formation events (as defined in the materials and methods section) visualized using MAP65a-citrine for wild-type and two $\Delta$ kin4-la mutant lines. The snapshots depicted above show the overlaps at their maximum observed length. The scheme on the right shows how measurements and calculations were performed to obtain the overlap extension rates depicted in $\mathrm{D}$. Images are maximum z-projections of 3 planes spaced $0.5 \mu \mathrm{m}$ apart taken in the cortical area. Horizontal scale bar, $1 \mu \mathrm{m}$; vertical scale bar, 30s. (D) Boxplots of the overlap extension rate measured in wild-type and two $\Delta$ kin4-la mutant strains expressing MAP65a-citrine. The whiskers indicate minimum / maximum observed values or 1.5 times the interquartile range when outliers (circles) were present. Differences are supported with ${ }^{*} \mathrm{P}<0.05$ and ${ }^{* *} \mathrm{P}<0.001$ from a Kruskal-Wallis test with pairwise comparisons.

mechanism results in a relatively sudden shut down of growth as opposed to a gradual or persistent downregulation of microtubule growth within overlaps. The time needed for growth termination could in this scenario be set by the delay in Kin4-Ia recruitment to overlaps and the time needed for plus end accumulation driven by motor activity (Figure 2).

The termination of growth within overlaps by Kin4-Ia could serve to keep the extension of microtubules pointing in the retrograde direction at a minimum, and thereby aid in polarity maintenance. Since the co-visualization of microtubule plus ends and overlaps could yield a coarse understanding on the nature and direction of microtubule-microtubule encounters in the unipolarized array of tip growing caulonema, we quantified the number of overlap formation events with an associated EB1b-comet travelling away from the cell apex (retrograde), towards the apex (anterograde), with two associated comets traveling in both directions and events 
without association (Figure 4C-D). This categorization revealed that under wild-type conditions overlap formation was mainly associated with a single EB1b-comet travelling in the retrograde direction, indicative of a plus end growing away from the apex colliding with the lattice of a microtubule pointing towards the apex (Figure 4E). Interestingly, also a substantial fraction of overlap formation events were observed that did not exhibit EB1b-mCherry association, suggesting that processes other than polymerisation also drive antiparallel bundling (e.g. relative microtubule-microtubule sliding (Fink et al. 2009)) or that EB1b-labelling is not always at detectable levels. Compared to wild-type, EB1b-association was more frequently seen in the $\Delta$ kin4-Ia genetic background (89\% of all events compared to $53 \%$ in wild-type; Figure 4E). Of all EB1b-asssociated events, the relative contribution of anterograde encounters and these involving growing ends from both directions increased from $\sim 14 \%$ to $\sim 33 \%$ in the Kin $4-\mathrm{Ia}$ mutant. This indicates that in absence of Kin4-Ia growing microtubule ends have a higher

A

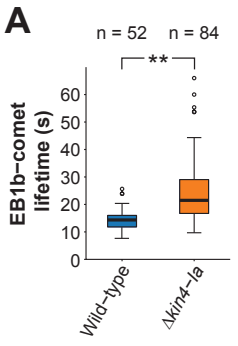

B

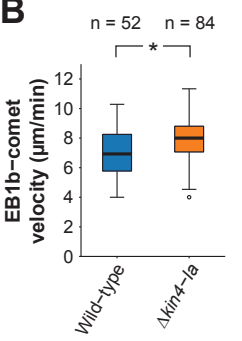

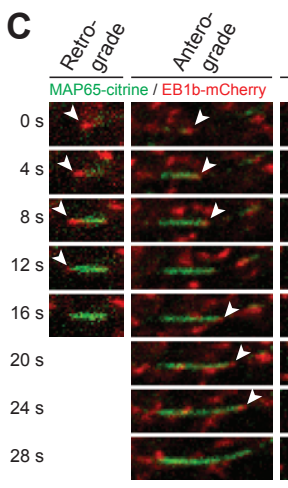
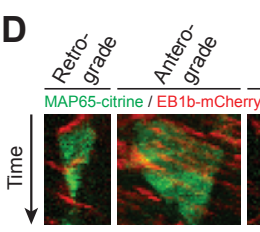

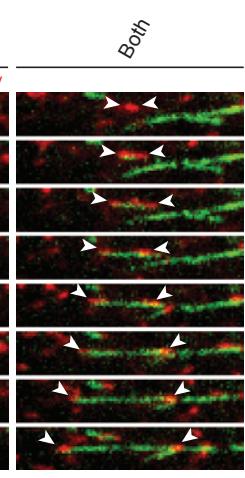

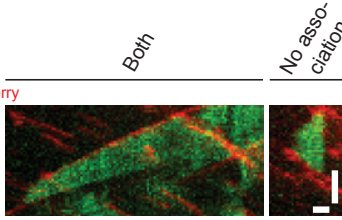

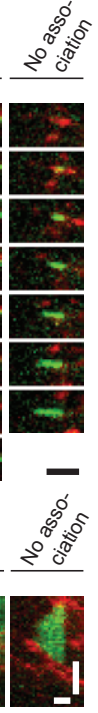

E

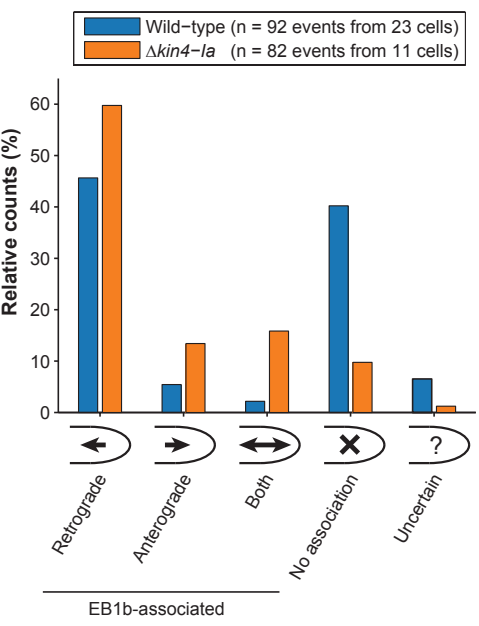

Figure 4 - Kin4-la limits overlap extension through inhibition of microtubule polymerisation. (A) Boxplots of EB1b-comet lifetimes in overlaps of wild-type and $\Delta k i n 4-l a$ cells. Comet lifetime was defined as the time during which an EB1b-comet associated with one side of an expanding microtubule overlap. The indicated number of EB1b-comets were measured. The whiskers indicate minimum I maximum observed values or 1.5 times the interquartile range when outliers (circles) were present. **Difference supported with $\mathrm{P}<0.001$ in a Mann-Whitney $\mathrm{U}$ test. (B) Boxplots of EB1b-comet velocities in overlaps of wild-type and $\Delta k i n 4-l a$ cells for the indicated number of EB1b-comets. The whiskers indicate minimum / maximum observed values or 1.5 times the interquartile range when outliers (circles) were present. *Difference supported with $\mathrm{P}<0.05$ in Student's $t$-test. (C) Time sequences of microtubule overlap formation events visualized with MAP65-citrine and microtubule plus end marker EB1b-mCherry (indicated with arrowheads). The events with EB1b-mCherry association were further categorised according to the growth direction of the microtubule plus end with respect to the cell apex: Retrograde (away from cell apex), anterograde (towards cell apex) or both (1 plus-end in each direction). The shown retrograde event was acquired in a wild-type background, the other events in a $\Delta$ kin4-la background. Images are maximum z-projections of 2 planes spaced $0.5 \mu \mathrm{m}$ apart acquired in the cortical area. Scale bar, $2 \mu \mathrm{m}$. (D) Kymographs of the microtubule overlap formation events depicted in C. Note that a larger section is shown. The categorisation of events is described in C. Horizontal scale bar, $1 \mu \mathrm{m}$; vertical scale bar, $30 \mathrm{~s}$. (E) Bar diagram showing relative occurrence of the different EB1b-association categories (as illustrated in C) obtained from the indicated number of overlap formation events in wild-type and $\Delta$ kin4-la cells. In case EB1b-association could not be unequivocally determined (e.g. due to high EB1b-comet abundance) the 'uncertain' category was assigned. 
propensity to interact with microtubules of opposite polarity and that the directional bias for retrograde encounters diminishes. This in turn hints that the activity of Kin4-Ia at overlaps has consequences for the overall topology of the microtubule network.

To assess the impact of overlap growth restriction by Kin4-Ia on the overall organization and polarization of the microtubule array we characterized the direction of all imaged EB1b-comets present in the movies used for the overlap-EB1b association assay using a particle tracking workflow (Figures 5A and S3B) (Jaqaman et al. 2008). This revealed a unimodal distribution of plus end growth trajectories centred around the basal-to-apical axis of the cell (set to $0^{\circ}$ ) in the wild-type situation. In contrast, a small second peak centred around the opposite direction was present in the distribution of the $\Delta$ kint-Ia mutant (Figure 5B arrowheads). This suggests that in absence of Kin4-Ia, enhanced growth in retrograde direction causes a bipolarisation of the microtubule array, although a net microtubule polarization towards the apex is maintained. Thus, Kin4-Ia's growth inhibiting activity at overlaps aids in overall array polarization, but is not required to establish or maintain the dominating direction. We found no differences in the speeds of EB1b-comets between wild-type and the $\Delta$ kin4-Ia mutant (Figure S3C). The mild difference in speed found for overlap-associated EB1b-comets (Figure 4B) is overall most likely drowned out by a majority of non-overlap-associated EB1b-comets that are not affected by Kin4-Ia.

Alongside the increase in retrograde oriented microtubule polymerization in the $\Delta$ kint-Ia mutant, EB1b-comet trajectories with angles close to $0^{\circ}$ (i.e. trajectories pointing exactly towards the cell tip) were also overrepresented (Figure 5B arrow). In the wild-type situation, polymerisation angles diverged near the apex with respect to the other areas of the cell (Figure 5C). However, in mutant cells at $\sim 10 \mu \mathrm{m}$ from the tip a subtle but notable continued alignment of polymerisation angles with the basal-to-apical cell axis was observed (Figure 5C). This could explain why the overall distribution of polymerization angles in $\Delta$ kin $4-I a$ cells peaked more sharply around $0^{\circ}$, despite the mild bipolarization of the array. How could this stricter alignment with the cell axis in the mutant situation arise? The unconstrained polymerization within overlaps mostly formed by retrograde travelling plus-ends (Figure 4) will result in more microtubule polymer length pointing in the basal direction. This in turn would increase the chance of anterograde growing plus ends having antiparallel encounters and consequent MAP65-mediated bundling with these microtubules. This is supported by the more frequent formation of overlaps associated with EB1b-comets traveling in anterograde or both anterograde and retrograde direction in the $\Delta$ kin4-Ia mutant (Figure 4E). Since the absence of Kin4-Ia would likewise not restrict the extension of this encounter, polymerization occurs unhindered in the exact opposite direction. Since this phenomenon universally occurs in both directions, it enhances both the orientation naturally most abundant and its opposite orientation. Since the MAP65-labelled regions of interaction between microtubules of these two directions are well-observable structures, we used these as a proxy to test if overall microtubule network alignment occurred in absence of Kin4-Ia. For this we recorded the angle with respect to the main cell axis of all overlaps analysed earlier for their length (Figure 2A). This indeed demonstrated that the orientation of overlaps became much more narrowly distributed around the cell axis upon removal of Kin4-Ia (Figure S4A). Thus, the limitation of overlap length between encountering antiparallel microtubules is a mechanism that, besides promoting a net basal-to-apical polarity of the array, also fosters polymerisation angle divergence.

To further understand the consequences for the overall alignment of the microtubule network 
with the cell axis upon loss of Kin4-Ia function we visualized the entire microtubule population by expression of a mCherry- $\alpha$-tubulin probe that did not alter colony size or appearance (Figure S4B). In the cortical cytoplasm of the $\Delta k i n 4-I a$ mutant a stronger longitudinal alignment of microtubules was apparent compared to the wild-type situation (Figure 5D). This was also the case in the median plane of the cell where we further noticed that vacuoles were more fragmented

A

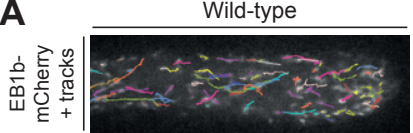

C

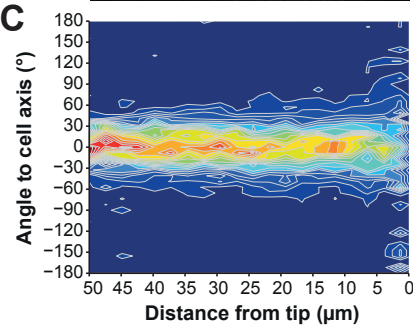

D

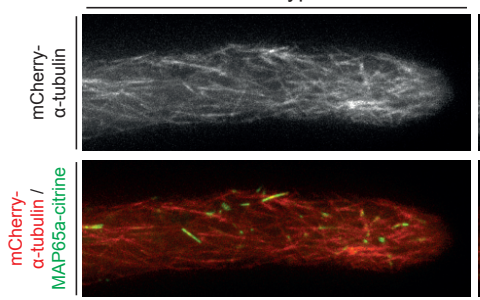

$\Delta$ kin4-la
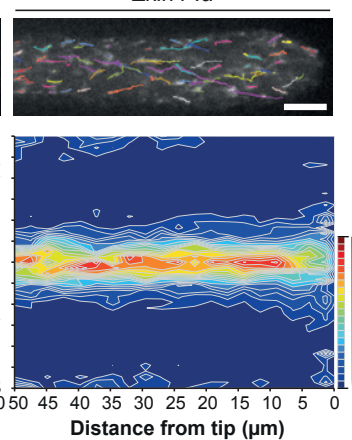

$\Delta$ kin4-la

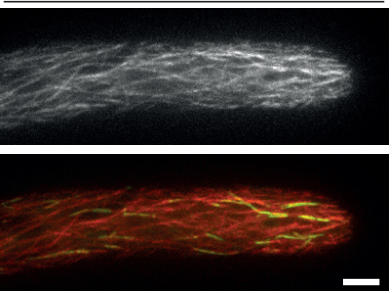

B

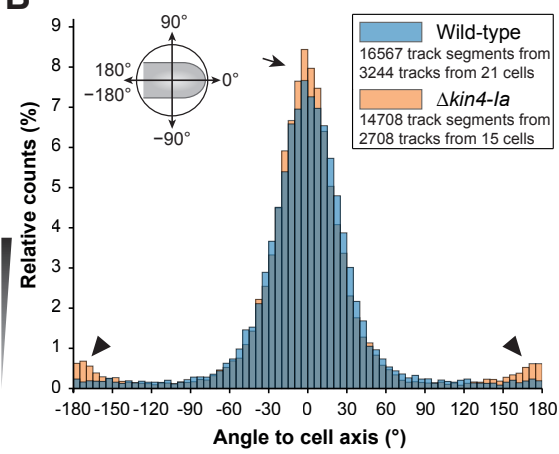

E

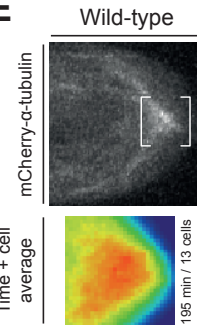

$\Delta$ kin4-la

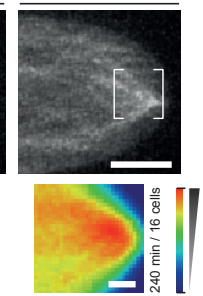

Figure 5 - Loss of Kin4-la leads to bipolarisation and hyper-parallelization of the interphase microtubule array in caulonemal tip cells. (A) Caulonemal tip cells expressing EB1b-mCherry together with the computationally identified tracks of microtubule plus ends labelled by this probe in a wild-type and $\Delta$ kin4-la mutant background. One frame from a time sequence is shown. The active tracks corresponding to this frame (i.e. tracks of which an identified comet in this frame is part of) are plotted in varying colours. The image is a maximum z-projection of 2 planes spaced $0.5 \mu \mathrm{m}$ apart acquired near the cell cortex. Scale bar, $5 \mu \mathrm{m}$. (B) Histogram showing the angle distribution for the indicated number of track-segments for wild-type and $\Delta$ kin4-la cells. Compared to wild-type, loss of Kin4-la leads to a larger fraction of EB1b-comets moving towards the base of the cell (arrowheads) and causes the comets moving towards the tip to be more aligned to the cell axis (arrow). (C) Contour plots with colour map showing the track-segment angle distribution as a function of distance to the cell tip for wild-type and $\Delta$ kin4-la cells (the same tracks were used as in B). The slightly higher abundance of track-segments with an angle close to that of the cell axis upon Kin4-la removal is noticeable from 50 until $\sim 10 \mu \mathrm{m}$ from the tip. (D) Microtubule array configuration in caulonemal tip cells visualized through expression of mCherry-a-tubulin in a wild-type and $\Delta$ kin4-la background. An overlay with the corresponding MAP65a-citrine signal is given below. Images shown are maximum z-projections of 9 planes spaced $0.27 \mu \mathrm{m}$ apart acquired near the cell cortex. Scale bar, $5 \mu \mathrm{m}$. (E) Snapshots showing the microtubule configuration in the apex of growing caulonemal tip cells visualized through mCherry-a-tubulin expression in a wild-type and $\Delta$ kin4-la genetic background. In both strains microtubules form a coalescing focus close to the dome of the apex. Snapshots are maximum z-projections of 10 planes spaced $0.5 \mu \mathrm{m}$ apart acquired around the median plane. Brackets delineate the areas that were used for generating the heat maps of the time and cell averaged apical microtuble intensity shown below. In brief, these were obtained by first computationally immobilizing growing cell tips, then making an average projection of image data acquired over 15 minutes and subsequently averaging over the indicated amount of cells. The average apical microtubule distribution appeared both more concentrated and more tapered upon loss of Kin4-la compared to wild-type. Scale bar in the top images, $5 \mu \mathrm{m}$; scale bar in the bottom images $1 \mu \mathrm{m}$. 
parallel to the main cell axis in the mutant situation (Figure S4C-D). Since the distribution of the vacuole in P. patens depends on microtubules (Oda et al. 2009), this is most likely the result of the higher degree of network parallelization.

The elucidated role of Kin4-Ia in preventing hyper-alignment of the microtubule network in apical cells does not yet provide a clear explanation for the tip growth steering phenotype (Figure 1D-G). To further investigate how these phenomena could be related we looked in more detail at the apical microtubule focus, a structure shown to consist of a bundle of coalescing microtubules that is involved in apical cell expansion (Hiwatashi et al. 2014) (Figure 5E). Due to the dynamic, cyclical appearance and disappearance of the apical microtubule focus (Hiwatashi et al. 2014), we followed it in multiple growing cells for $15 \mathrm{~min}$ and then averaged the mCherry- $\alpha$-tubulin signal to extract its dominant shape and residence characteristics (Figure 5E bottom). This revealed that upon Kin4-Ia removal a more tapered and more sharply defined area containing high average microtubule density was present, indicating the stronger microtubule alignment impacts the apical microtubule focus. The sharper focussing of this structure could account for the more stringent growth axis observed in $\Delta$ kint-Ia mutants. However, the poorly understood molecular makeup of this structure and lack of mechanistic insight on how it provides spatial cues for the growth process currently hamper conclusions about a direct connection between microtubule array structure and tip growth guidance. Nonetheless, the length scales on which microtubule networks exist in plant tip growing cells and how they contribute to cell polarity during apical growth in fungi, make microtubule plus end-mediated spatial cues for persistent growth an attractive concept (Sieberer et al. 2005; Ishitsuka et al. 2015). A key question to answer is thus how microtubule network organization is coupled to growth processes. Future investigation of components connected to the apical microtubule focus should have special regard for the actin cytoskeleton because many tip growing cells, including P. patens protenamata, have an accumulation of dynamic actin present at the tip (Vidali et al. 2009; Rounds \& Bezanilla 2013; Ketelaar 2013). Furthermore, crosstalk between the microtubule and actin cytoskeleton during tip growth has been proposed in tip growing pollen tubes (Justus et al. 2004; Zhu et al. 2013).

In this work we show that the concentration of a growth-inhibiting regulator at microtubule overlaps constitutes a mechanism to both aid in array polarization and at the same time preventing the array from becoming hyper-parallelized to the longitudinal cell axis. As such, antiparallel bundling by MAP65 can not only be used to locally reinforce array orientation (Wasteneys \& Ambrose 2009), but to reinforce polarity as well through recruitment of a secondary factor. This principle constitutes a novel tool in the plant toolbox to pattern dispersedly nucleated arrays and a similar mechanism may account for locally polarised sectors in the higher plant cortical array (Dixit et al. 2006). Here we only found a role for Kin4-Ia in preventing overlap elongation. If and how these overlaps are then subsequently actively removed remains to be investigated. Interestingly, the elongated overlaps in the $\Delta$ kin 4 -Ia mutant showed occasional splitting in the middle part of the overlap, reminiscent of microtubule severing (Figure 3C) (Burk et al. 2007). Thus, besides a more obvious activity such as depolymerisation, severing should also be considered to contribute to overlap disappearance. It will be intriguing to further learn how overlap-based activities together with other control- and reorganising systems build a polarised microtubule network that supports robust, yet adaptable tip growth. 


\section{Materials and Methods}

\section{Plasmids and cloning procedures}

Plasmids used were generated using standard restriction-ligation cloning procedures and were verified by sequencing. The use of plasmids is summarized in table 2 and all primers are listed in table 1.

Table 1 - List of primers used in this study

\begin{tabular}{|c|c|c|c|}
\hline Primer & Use & Sequence (5'> 3') & RE site \\
\hline JK206 & Kin4-la deletion genotyping & TTTGAGGTGTATCACCCAGTTTCG & - \\
\hline JK207 & Kin4-la citrine tagging genotyping & GGAGGAAAGTGATGGCGAAAGAGC & - \\
\hline JK208 & Kin4-la deletion / citrine tagging genotyping & GCAGGAAGCACGAGCTAAGACC & - \\
\hline JK161 & Genotyping of MAP65a locus & GGATGAGAACCGATTTGCGAGC & - \\
\hline JK162 & Genotyping of MAP65a locus & GCGGTAAGAAAGTGGAGAGAAGC & - \\
\hline JK87 & Cloning of EB1b left targeting arm & GGGGTACCCAGCTGATGGACGTAGTGCACC & Kpnl \\
\hline JK88 & Cloning of EB1b left targeting arm & CCСAAGCTTCTCTAAAAGATCCTTACTGTCGTCG & HindllI \\
\hline JK124 & Cloning of EB1b right targeting arm & TGTTGACGGCACTATTACTCGC & - \\
\hline JK90 & Cloning of EB1b right targeting arm & TCCCCGCGGTATGGACACTCACCAGGCAACC & Sacll \\
\hline JK114 & Sequence downstream of resistance cassette & ACTCAAGAGGATAAAACCTCACC & - \\
\hline JK111 & Construct sequencing general & GATTAAGTTGGGTAACGCCAGG & - \\
\hline JK116 & Construct sequencing general & ACACAGGAAACAGCTATGACC & - \\
\hline JK129 & Sequencing region upstream of mCherry & CCCTTGGTCACCTTCAGCTTGG & - \\
\hline JK156 & Genotyping of EB1b locus & CATTTGTGGGTATGTGGGTTGG & - \\
\hline JK157 & Genotyping of EB1b locus & TGACAAAGGGAGAATAAAAGAGGTGG & - \\
\hline JK210 & Kin4-Ic citrine tagging genotyping & ACTGACCTGCCACATTCCGAGC & - \\
\hline JK211 & Kin4-Ic citrine tagging genotyping & TTGACTTGTGTGGAATCTTGGATGC & - \\
\hline
\end{tabular}

\section{EB1b-mCherry-BsdR}

A $\sim 1.2 \mathrm{~kb}$ region downstream of the EB1b gene stopcodon was amplified using primers JK124 and JK90, digested with SacII and ligated into SmaI / SacII digested pmCherry-LoxPBsdR (de Keijzer et al. 2017). A $\sim 1.1 \mathrm{~kb}$ region upstream of the stop codon was subsequently amplified using primers JK87 and JK88 and introduced via KpnI and HindIII sites. The resulting tagging construct was digested with $S m a \mathrm{I}$ and $\mathrm{SacII}$ before transformation.

Table 2 - List of plasmids used in this study

\begin{tabular}{|c|c|c|}
\hline Plasmid & Usage & Source \\
\hline MAP65a-mCherry & C-terminal mCherry tagging of MAP65a & $\begin{array}{l}\text { (de Keijzer et al. } \\
2017 \text { ) }\end{array}$ \\
\hline EF1-mCherry-tua-BsdR (pTM426) & EF1 $\alpha$-promoter driven expression of mCherry- $\alpha$-tubulin & $\begin{array}{l}\text { (T. Miki, K. Kosetsu } \\
\text { and G. Goshima, } \\
\text { unpublished) }\end{array}$ \\
\hline EB1b-mCherry & C-terminal mCherry tagging of expressed EB1-homologue EB1b & this study \\
\hline pTM374 & C-terminal citrine tagging of Kin4-la & (Miki et al. 2014) \\
\hline pTM365 & C-terminal citrine tagging of Kin4-lc & (Miki et al. 2014) \\
\hline
\end{tabular}




\section{P. patens growth conditions and transformation}

$P$. patens tissues were routinely grown on BCDAT plates under continuous light, unless stated otherwise. Plasmids were linearized and introduced into the $P$. patens genome by homologous recombination using PEG-mediated protoplast transformation (Nishiyama et al. 2000). Correct insertion events were characterised by PCR. Characteristics of generated moss lines and their use throughout the study are summarized in table 3. For imaging, protonemal tissue was grown on BCD medium in glass bottom dishes.

Table 3 - List of moss strains used in this study

\begin{tabular}{|c|c|c|c|c|c|c|}
\hline Name & Experimental use & $\begin{array}{l}\text { Main } \\
\text { clone } \\
\text { used }\end{array}$ & Background & Plasmid & Figure & Reference \\
\hline Kin4-la-citrine & $\begin{array}{l}\text { Generating kin4-la + antiparallel } \\
\text { overlap colocalization strain }\end{array}$ & \#43 & WT & pTM374 & - & $\begin{array}{l}\text { (Miki et al. 2014; } \\
\text { de Keijzer et al. } \\
2017 \text { ) }\end{array}$ \\
\hline $\begin{array}{l}\text { Kin4-la-citrine / } \\
\text { MAP65a-mCherry }\end{array}$ & $\begin{array}{l}\text { Kinesin4-la + antiparallel overlap } \\
\text { colocalization }\end{array}$ & \#121 & Kin4-la-citrine & $\begin{array}{l}\text { MAP65a- } \\
\text { mCherry }\end{array}$ & $\begin{array}{l}1 \mathrm{~B}-\mathrm{H} \\
\mathrm{S} 2 \mathrm{~A} \\
\mathrm{~S} 2 \mathrm{C}\end{array}$ & This study \\
\hline MAP65a-citrine & $\begin{array}{l}\text { Overlap visualization / kin4-la } \\
\text { knock-out construction }\end{array}$ & \#2 & WT & $\begin{array}{l}\text { see refer- } \\
\text { ence }\end{array}$ & $\begin{array}{l}2,5 \\
\text { S2B } \\
\text { S4D }\end{array}$ & $\begin{array}{l}\text { (Kosetsu et al. } \\
\text { 2013) }\end{array}$ \\
\hline $\begin{array}{l}\text { MAP65a-citrine / } \\
\text { Skin4-la }\end{array}$ & $\begin{array}{l}\text { Measuring effect of kin4-la removal } \\
\text { on overlap dimension and cell tip } \\
\text { growth }\end{array}$ & $\begin{array}{l}\# 7 \& \\
\# 122\end{array}$ & $\begin{array}{l}\text { MAP65a-cit- } \\
\text { rine }\end{array}$ & $\begin{array}{l}\text { see refer- } \\
\text { ence }\end{array}$ & $\begin{array}{l}2,5 \\
\text { S2B, } \\
\text { S4D }\end{array}$ & $\begin{array}{l}\text { (de Keijzer et al. } \\
2017 \text { ) }\end{array}$ \\
\hline MAP65c-citrine & $\begin{array}{l}\text { Generating overlap + EB1b - colo- } \\
\text { calization strain }\end{array}$ & \#5 & WT & $\begin{array}{l}\text { see refer- } \\
\text { ence }\end{array}$ & - & $\begin{array}{l}\text { (Kosetsu et al. } \\
\text { 2013) }\end{array}$ \\
\hline $\begin{array}{l}\text { MAP65c-citrine / } \\
\text { EB1b-mCherry }\end{array}$ & $\begin{array}{l}\text { Polymerisation visualization in } \\
\text { overlaps / plus-end tracking }\end{array}$ & \#100 & MAP65c-citrine & $\begin{array}{l}\text { EB1b- } \\
\text { mCherry }\end{array}$ & $\begin{array}{l}3,4 \mathrm{~A}- \\
\mathrm{C}, \mathrm{S3}\end{array}$ & This study \\
\hline $\begin{array}{l}\text { MAP65a-cit- } \\
\text { rine / } \Delta \text { kin4-la / } \\
\text { EB1b-mCherry }\end{array}$ & $\begin{array}{l}\text { Measuring effect of kin4-la removal } \\
\text { on polymerisation dynamics in over- } \\
\text { laps and orientation of polymerisa- } \\
\text { tion in general }\end{array}$ & \#96 & $\begin{array}{l}\text { MAP65a-cit- } \\
\text { rine / } \Delta \text { kin4-la } \\
\# 7\end{array}$ & $\begin{array}{l}\text { EB1b- } \\
\text { mCherry }\end{array}$ & $\begin{array}{l}3,4 \mathrm{~A}- \\
\mathrm{C}, \mathrm{S3}\end{array}$ & This study \\
\hline $\begin{array}{l}\text { MAP65a-citrine / } \\
\text { mCherry- } \alpha \text {-tubulin }\end{array}$ & $\begin{array}{l}\text { Microtubule cytoskeleton + antipar- } \\
\text { allel overlap visualization }\end{array}$ & $\# 426$ & $\begin{array}{l}\text { MAP65a-cit- } \\
\text { rine }\end{array}$ & $\begin{array}{l}\text { EF1-mCher- } \\
\text { ry-tua-BsdR }\end{array}$ & $\begin{array}{l}\text { 1A, 4D- } \\
E, S 1 A \\
\text { S4A-C }\end{array}$ & This study \\
\hline $\begin{array}{l}\text { MAP65a-citrine / } \\
\Delta \text { kin4-la / mCher- } \\
\text { ry-a-tubulin }\end{array}$ & $\begin{array}{l}\text { Measuring effect of kin4-la removal } \\
\text { on MT-network architecture and } \\
\text { apical focussing }\end{array}$ & $\# 14$ & $\begin{array}{l}\text { MAP65a-cit- } \\
\text { rine / } \Delta \text { kin4-la } \\
\# 7\end{array}$ & $\begin{array}{l}\text { EF1-mCher- } \\
\text { ry-tua-BsdR }\end{array}$ & $\begin{array}{l}4 D-E \\
S 4 A-C\end{array}$ & This study \\
\hline $\begin{array}{l}\text { EF1-mCherry-a-tu- } \\
\text { bulin }\end{array}$ & $\begin{array}{l}\text { Generating kin4-la / kin4-lc + micro- } \\
\text { tubule array colocalization strains }\end{array}$ & \#52 & WT & $\begin{array}{l}\text { EF1-mCher- } \\
\text { ry-tua-BsdR }\end{array}$ & - & $\begin{array}{l}\text { (K. Kosetsu, } \\
\text { unpublished) }\end{array}$ \\
\hline $\begin{array}{l}\text { EF1-mCherry-a-tubu- } \\
\text { lin / Kin4-la-citrine }\end{array}$ & $\begin{array}{l}\text { Localization screen of all } P \text {. patens } \\
\text { kinesin- } 4 s \text { in caulonemal tip cells }\end{array}$ & \#22 & $\begin{array}{l}\text { EF1-mCher- } \\
\text { ry-a-tubulin }\end{array}$ & pTM374 & S1B & $\begin{array}{l}\text { This study; (Miki } \\
\text { et al. 2014) }\end{array}$ \\
\hline $\begin{array}{l}\text { Act-mCherry- } \alpha \text {-tubu- } \\
\text { lin / Kin4-lb-citrine }\end{array}$ & $\begin{array}{l}\text { Localization screen of all } P \text {. patens } \\
\text { kinesin- } 4 \text { s in caulonemal tip cells }\end{array}$ & \#12 & $\begin{array}{l}\text { Act-mCher- } \\
\text { ry-a-tubulin }\end{array}$ & $\begin{array}{l}\text { see refer- } \\
\text { ence }\end{array}$ & S1B & (Miki et al. 2014) \\
\hline $\begin{array}{l}\text { EF1-mCherry-a-tubu- } \\
\text { lin / Kin4-Ic-citrine }\end{array}$ & $\begin{array}{l}\text { Localization screen of all } P \text {. patens } \\
\text { kinesin- } 4 \mathrm{~s} \text { in caulonemal tip cells }\end{array}$ & \#19 & $\begin{array}{l}\text { EF1-mCher- } \\
\text { ry-a-tubulin }\end{array}$ & pTM365 & S1B & $\begin{array}{l}\text { This study; (Miki } \\
\text { et al. 2014) }\end{array}$ \\
\hline $\begin{array}{l}\text { Act-mCherry- } \alpha \text {-tubu- } \\
\text { lin / Kin4-Id-citrine }\end{array}$ & $\begin{array}{l}\text { Localization screen of all } P \text {. patens } \\
\text { kinesin- } 4 \text { s in caulonemal tip cells }\end{array}$ & \#8 & $\begin{array}{l}\text { Act-mCher- } \\
\text { ry-a-tubulin }\end{array}$ & $\begin{array}{l}\text { see refer- } \\
\text { ence }\end{array}$ & S1B & (Miki et al. 2014) \\
\hline $\begin{array}{l}\text { Act-mCherry-a-tubu- } \\
\text { lin / Kin4-le-citrine }\end{array}$ & $\begin{array}{l}\text { Localization screen of all } P \text {. patens } \\
\text { kinesin- } 4 \mathrm{~s} \text { in caulonemal tip cells }\end{array}$ & $\# 14$ & $\begin{array}{l}\text { Act-mCher- } \\
\text { ry-a-tubulin }\end{array}$ & $\begin{array}{l}\text { see refer- } \\
\text { ence }\end{array}$ & S1B & (Miki et al. 2014) \\
\hline $\begin{array}{l}\text { Act-mCherry-a-tubu- } \\
\text { lin / Kin4-Ila-citrine }\end{array}$ & $\begin{array}{l}\text { Localization screen of all } P \text {. patens } \\
\text { kinesin- } 4 \mathrm{~s} \text { in caulonemal tip cells }\end{array}$ & \#2 & $\begin{array}{l}\text { Act-mCher- } \\
\text { ry-a-tubulin }\end{array}$ & $\begin{array}{l}\text { see refer- } \\
\text { ence }\end{array}$ & S1B & (Miki et al. 2014) \\
\hline $\begin{array}{l}\text { Act-mCherry-a-tubu- } \\
\text { lin / Kin4-Ilb-citrine }\end{array}$ & $\begin{array}{l}\text { Localization screen of all } P \text {. patens } \\
\text { kinesin- } 4 \text { s in caulonemal tip cells }\end{array}$ & \#5 & $\begin{array}{l}\text { Act-mCher- } \\
\text { ry-a-tubulin }\end{array}$ & $\begin{array}{l}\text { see refer- } \\
\text { ence }\end{array}$ & S1B & (Miki et al. 2014) \\
\hline $\begin{array}{l}\text { Act-mCherry-a-tubu- } \\
\text { lin / Kin4-Ilc-citrine }\end{array}$ & $\begin{array}{l}\text { Localization screen of all } P \text {. patens } \\
\text { kinesin-4s in caulonemal tip cells }\end{array}$ & $\# 9$ & $\begin{array}{l}\text { Act-mCher- } \\
\text { ry-a-tubulin }\end{array}$ & $\begin{array}{l}\text { see refer- } \\
\text { ence }\end{array}$ & S1B & (Miki et al. 2014) \\
\hline
\end{tabular}

\section{Gravistimulation of moss colonies}

P. patens colonies were initiated from small protonemal fragments on BCDAT plates supplemented with $0.5 \%(\mathrm{w} / \mathrm{v})$ glucose and allowed to grow for 2 weeks under continuous 
light. Plates were then put in an upright position in the dark for 5 weeks. Outgrowth from the colonies was then photographed with a Bio-Rad ChemiDoc XRS+ imaging system using transillumination.

\section{Fluorescence microscopy}

In all fluorescence microscopy experiments interphase caulonemal tip cells were used. Imaging was performed on a spinning disk confocal microscope consisting of a Nikon Ti-eclipse body equipped with a Yokogawa CSU-X1 spinning disk head and 100x Plan Apo VC objective (NA 1.40). Image digitization was either done with a Photometrics Evolve 512 EMCCD camera with a 1.2x post-magnification fitted in front of the camera or an Andor iXon 3 model 888 with a 2x post-magnification fitted in front of the camera. Typical exposures used were 500-1000ms while employing a camera electron multiplication gain of 300. For imaging of citrine an excitation wavelength of $491 \mathrm{~nm}$ in combination with a 527/60 bandpass emission filter was used and for imaging of mCherry $561 \mathrm{~nm}$ excitation light in combination with a 595/50 or 607/36 bandpass emission filter was used. All components were operated by MetaMorph software.

For synchronized imaging of MAP65 labelled with citrine and mCherry-tagged EB1b the excitation light for both probes was delivered simultaneously and their emission light was first filtered (495-550nm and $>575 \mathrm{~nm}$ ) and then split using a Photometrics DV2 Multichannel Imaging System. Beam-splitting was achieved with a T565lpxr dichroic mirror (Chroma) with additional bandpass filtering at 495-540nm for citrine and at 570-620nm for mCherry.

\section{Long term imaging of tip growth}

Long term observation of tip growth was done with bright field microscopy using continuous sample illumination with white light from a Nikon D-LH/LC halogen lamp filtered through a NCB11 filter and a 10x Plan Fluor objective (NA 0.30). Images were captured with either a Photometrics Evolve 512 EMCCD camera with a 1.2x post-magnification fitted in front of the camera or directly with a Photometrics CoolSNAP HQ2 CCD camera.

\section{Image analysis}

All image analysis was performed using the FIJI distribution of Image 1.51 and custom written MATLAB scripts. Measurements were only taken throughout the first $50 \mu \mathrm{m}$ from the cell apex since this always excluded the nucleus in the observed cells. When dynamics of overlaps are presented only entire overlap formation events were used. An overlap formation event was defined as the de novo generation of a MAP65-labelled overlap region within the imaged observation plane(s) visible as an extending, bar-shaped signal. For display purposes all images in the manuscript were rotated (bicubic interpolation) such that the cell tip pointed to the right-hand side.

\section{MAP65a / Kin4-Ia colocalization assessment}

Z-stacks of MAP65a-mCherry and Kin4-Ia-citrine signals acquired along 20 planes spaced $0.4 \mu \mathrm{m}$ apart were first z-projected (maximum intensity). Then, MAP65a-mCherry labelled overlaps were identified and the presence of Kin4-Ia-citrine fluorescence at the same location was manually scored. When chloroplast autofluorescence obstructed colocalization assessment the original z-stack was consulted and when also here unequivocal assessment was impossible, an 'uncertain' category was assigned. 
Observation of MAP65a / Kin4-Ia dynamics

Overlap formation events were isolated and scored manually for the occurrence of a 'spur' of Kin4-Ia-citrine fluorescence away from the MAP65a-labelled overlap region. For measurements on Kin4-Ia linear motility within spurs overlap formation was first identified by monitoring of the MAP65a-mCherry signal after which the Kin4-Ia-citrine signal was acquired at high frame rate. Kymographs of the Kin4-Ia-citrine signal were generated using MetaMorph (Linewidth 5 pixels, using average value across width). The steepness and horizontal extension of lines in the resultant kymographs were used to determine particle velocity and runlength respectively.

Overlap angle, length and tip distance measurements

Z-stacks of overlaps labelled either with MAP65a-citrine or MAP65a-mCherry acquired along 20 planes spaced $0.4 \mu \mathrm{m}$ apart were first z-projected (maximum intensity). Along the length axis of continuous, bar-shaped signals a straight line segment was drawn in ImageJ of which length, angle and centroid were determined. Measured angles were subtracted with the angle of the longitudinal axis of the cell. The distance to the tip was defined as the horizontal distance between a line segment's centroid and the cell tip after aligning the longitudinal axis of the cell with the $\mathrm{x}$-axis of the image frame. Contour plots with colour map of the angle distribution of overlaps as a function of distance to the cell tip were made by first sorting data according to tip distance in $2 \mu \mathrm{m}$ bins. Then, for each distance bin a normalized, overlap length-weighted distribution of overlap angles divided in $12^{\circ}$ bins was generated.

Measurement of overlap extension rates

Kymograph representations of overlap formation events visualized with MAP65a-citrine were produced using the ImageJ KymographBuilder plugin. The generated kymographs were then used to measure the maximum acquired overlap length, as well as the time needed to reach this length. The maximum acquired length was defined as the length of the overlap at the moment before it would either stop extending, start shrinking or would be severed and break apart (also see scheme in figure 3C). For overlap extension rate calculation the maximum length was divided by the time taken to reach that length.

Categorisation of EB1b comet direction and measurement of comet dynamics in overlaps

EB1b-mCherry image data was first denoised using MetaMorph plugin Safir 1.0.3 in 2D+t mode (see also Figure S3B). The denoised EB1b image sequences were then overlaid with the co-visualized MAP65-labelled overlaps and overlap formation events were isolated. Kymographs of events were made for both signals using the KymographBuilder plugin in ImageJ. Scoring of the association of EB1b-comets with overlap formation including their direction relative to the cell tip was done by analysis of both overlaid time-lapse sequences and kymographs. The kymographs of overlap formation events with EB1b association were subsequently used to determine EB1b comet velocities and lifetimes. The steepness of the lines generated by EB1bcomets was used to obtain velocities and the time during which comets remained associated with the MAP65 signal was measured to obtain their lifetimes.

Tracking of EB1b-comets

The workflow and input parameters used for generating EB1b-comet trajectories and their subsequent analysis is schematically outlined in figure S3B.

An image sequence of EB1b-mCherry signal acquired at two confocal z-planes in the cell cortex was projected along the $z$-axis (maximum intensity). Noise in the resultant image sequence was then supressed using MetaMorph plugin Safir 1.0.3 in 2D+t mode (Boulanger et al. 2010). 
Owing to the relatively weak signal and use of electron multiplication gain in the imaging setup, we assumed photon shot noise to be the dominant noise component in the final image, therefore using 'Poisson' as the noise type.

Subsequently, to identify EB1b-comets against the cytoplasmic background signal a first segmentation step was performed by applying a global threshold obtained using the maximum entropy method employing 500 bins in the intensity histogram (Kapur et al. 1985). As the resulting areas were often composed of multiple features further intensity-based segmentation was performed. For this, first the local intensity maxima generated by individual comets were enhanced by convolving the image with a Difference of Gaussians (DoG) kernel. This form of bandpass filtering uses a subtraction of two Gaussian kernels with different standard deviation $(\sigma)$. The first $\sigma$ was chosen based on a Gaussian approximation of the point spread function describing the lateral resolution limit of the microscope setup and fluorophore used $(0.68$ px; Zhang et al. 2007). The second $\sigma$ was selected such that the filter would give a maximum response at the length scale of a comet, thus preserving information in the range of comet spatial frequencies (Matov et al. 2010). As the measured average comet length was 4.4 pixels (n $= \pm 4300$ comets), $\mathrm{a} \sigma$ of 3.1 pixels was used.

Then, using the DoG convolved image data, intensity based segmentation was performed by stepwise application of an increasingly lower segmentation threshold within the areas identified with the global threshold earlier. In this way, intensity peaks are first detected which are then allowed to grow in each successive segmentation step until reaching the minimum intensity level or merging with a neighbouring peak. When two growing peaks would fuse together, the two original areas obtained at a higher segmentation threshold are retained, thereby allowing the detection of individual particles which were before classified as a single object peak using the global intensity threshold. The step size for intensity based segmentation was the three-frame average of intensity standard deviation obtained from one frame before till one after the current frame. A minimum of 2 steps was enforced for segmentation. After completing the intensity based segmentation only areas bigger than 2 pixels were retained.

Next, an intensity-weighted centroid for the finally accepted areas was determined using the intensities of the noise-supressed image data. The coordinates of these centroids were then passed to the 'robust single-particle tracking' framework published by Jaqaman et al. (2008). This code first links particles in a frame-to-frame manner based on a linear assignment problem. For this, a cost matrix containing the costs for potential links between particles in consecutive frames and the cost for birth and death of tracks is solved. The linking cost for particles is based on a prediction of a track's propagation in time calculated through a Kalman filtering procedure (Jaqaman et al. 2008). Since the tracks obtained from this step are usually incomplete due to limitations in imaging (e.g. comets temporarily moving out of the imaged focal planes), a second global optimization step, achieved through solving another linear assignment problem, is performed yielding the final tracks (Jaqaman et al. 2008).

Finally, the displacement characteristics of tracked particles were analysed. For this timeaveraged track segments were used to determine growth rates and the angle of displacement. Track segments spanned a particle's position spaced 5 frames apart. Displacement was determined with a time-average instead of on a frame-to-frame basis as this suppressed the adverse effects of the placement error of the intensity-weighted centroid. This error is introduced by the modulation transfer function of the used microscope setup and is enhanced by the confined area retained after segmentation. The recorded particle angles were corrected with the angle of 
the cell such that tip-ward particle motion was equal to $0^{\circ}$. Contour plots with colour map of the angle distribution of track segments as a function of distance to the cell tip were made by first sorting data according to tip distance in $2.5 \mu \mathrm{m}$ bins. Then, for each distance bin a normalized distribution of track segment angles divided in $8^{\circ}$ bins was generated.

\section{Analysis of apical microtubule structure in growing cells}

First, cell displacement caused by tip growth was countered by recording the position of the tip at the start and end of imaging. The inferred linear path between these points was used to translate the consecutive frames of the z-projected movie (maximum intensity) using an affine transform (bicubic interpolation) creating alignment with the first frame. Then, the apical area containing the microtubule focus was cropped to a $4 \times 4 \mu \mathrm{m}$ square, the corners of which touched the edge of the apical dome. The time-lapse image data in this area was subsequently projected (average intensity) for each imaged cell. The intensities of these average projections were normalised and then averaged per genotype.

\section{Tracking of tip growth}

From the long term time-lapse imaging series of caulonemal outgrowth, the individual areas containing the growing cell tips were obtained and subdivided in 4 hour time periods. Then, for each growth period the apex of the cell (defined as the place with the highest local curvature) was manually determined at 4 minute intervals using the point tool in Fiji. The centroid locations were then smoothed using locally weighted linear regression analysis (employing quadratic polynomial regression and a span width of $15 \%$ ). The obtained smoothened paths were then used for determination of the distance traversed by the tip and, by dividing this distance with the observation time, used to calculate the average growth speed. A simple linear regression analysis of the smoothened tip tracks was performed to obtain an idealized linear path for each growing tip, used to calculate the relative straightness of a tip's trajectory (see also figure 1F).

\section{Angle determination of gravistimulated colony outgrowth}

Filament angles were determined with the OrientationJ Distribution plugin (Püspöki et al. 2016) employing a Gaussian window standard deviation of 0.85 pixels, a minimum coherency of $10 \%$ and a minimum energy of $1 \%$. All obtained angles in the region spanning $2 \mathrm{~mm}$ at the circumference of the top of the colony were rounded and weighted with their background corrected pixel intensities. The intensity-weighed angle distributions obtained for twelve colonies were then averaged and displayed in an angular histogram composed of $12^{\circ}$ bins.

\section{Statistical analysis}

For statistical validation, data were first tested for normality using the Shapiro-Wilk test $(\alpha=0.05)$ verified by visual inspection of $\mathrm{Q}-\mathrm{Q}$ plots. When normality was assumed, data were evaluated using either an unpaired t-test ( 2 samples) or a one-way ANOVA analysis ( $>2$ samples). For data where a normal distribution was not assumed, either a Mann-Whitney U test (2 samples) or Kruskal-Wallis test with pairwise comparisons ( $>2$ samples) was employed. For all statistical analysis IBM SPSS Statistics version 22 or 23 was used. 


\section{Supplemental figures}

A

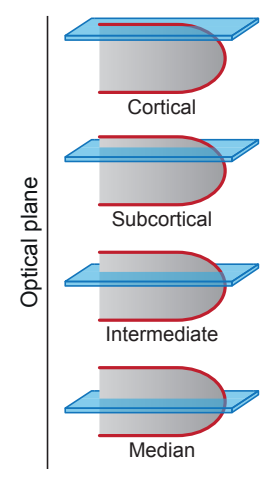

MAP65a-citrine

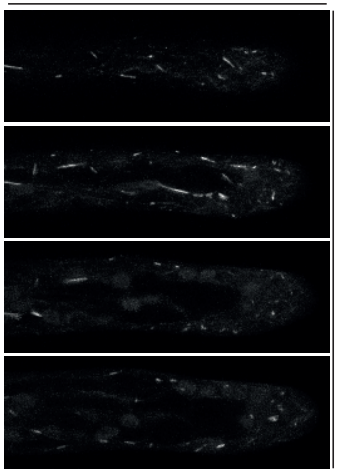

B

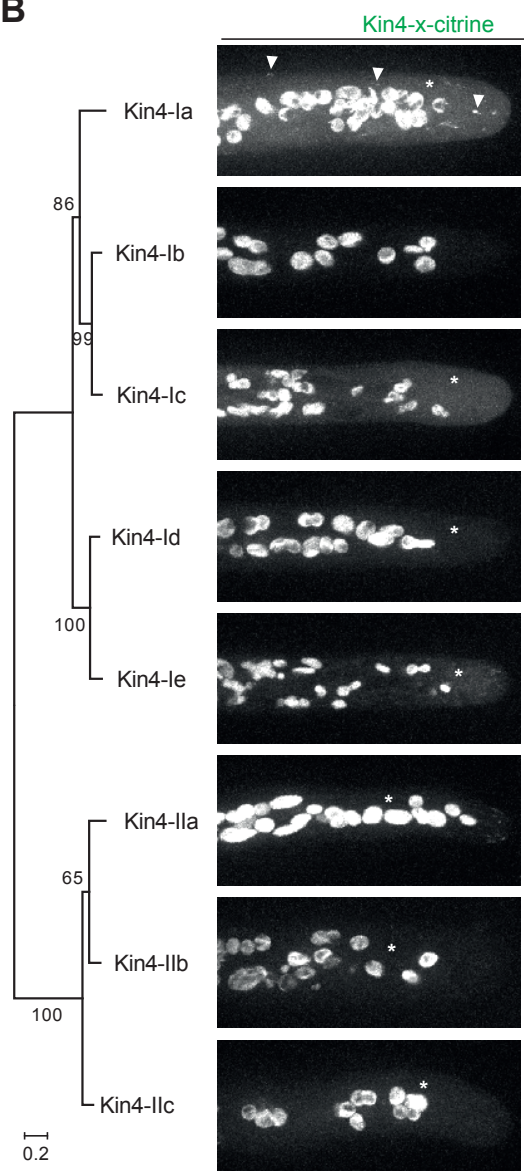

mCherry-a-tubulin
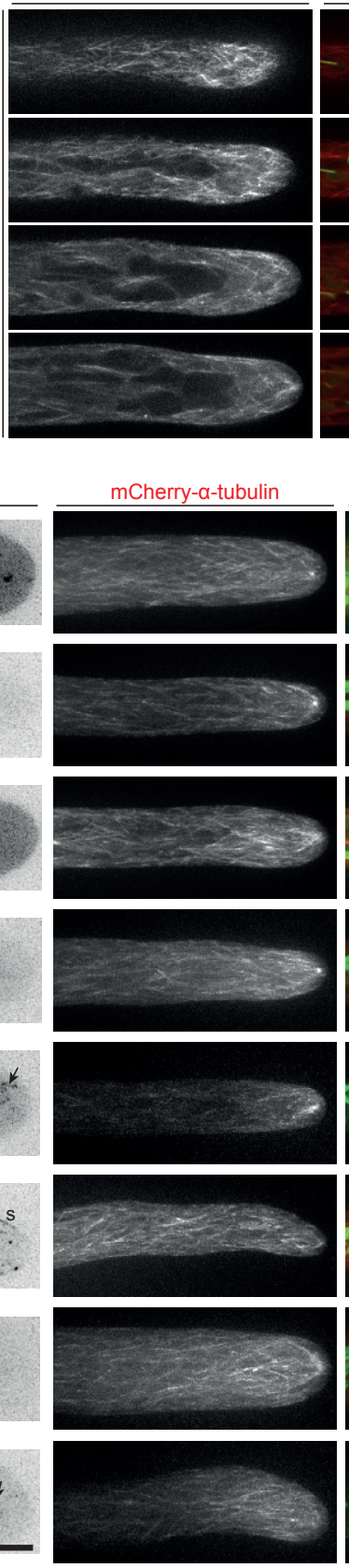

Merge
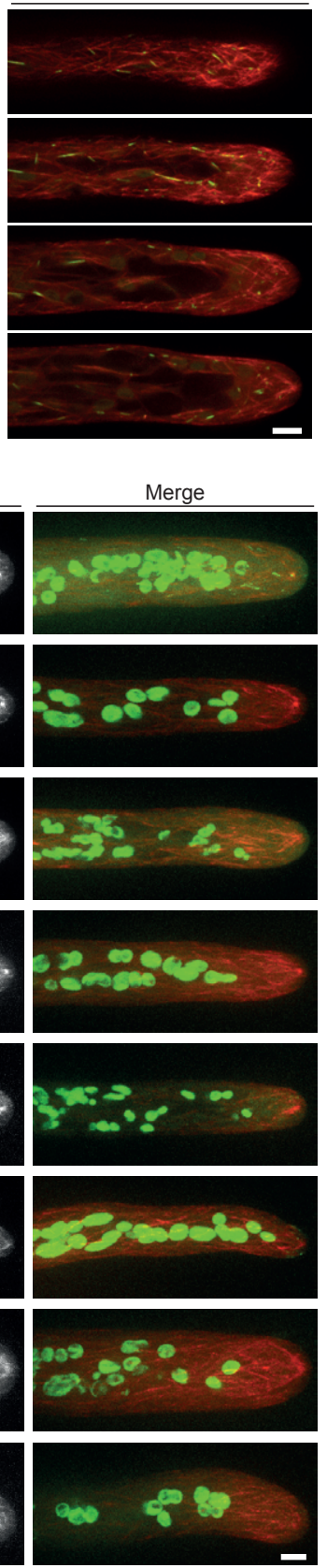

Figure S1 - 3D architecture of microtubule network in caulonemal tip cells and localization survey of $\boldsymbol{P}$. patens kinesin-4s. (A) Microtubule network architecture of a caulonemal tip cell divided in four serial optical planes as indicated on the left, visualized using a cell expressing $m$ Cherry- $\alpha$-tubulin and MAP65a-citrine. Microtubules and concomitant antiparallel overlaps occur throughout the cell. While absent near the cortex, larger organelles like vacuoles and chloroplasts become 
increasingly abundant towards the median plane of the cell. Shown images are maximum z-projections of 5 confocal planes spaced $0.4 \mu \mathrm{m}$ apart. Scale bar, $5 \mu \mathrm{m}$. (B) Localization survey of all eight kinesin-4s in P. patens caulonemal tip cells. All kinesin-4-citrine signals were acquired with identical illumination and detection settings and are shown using an equal intensity display range. Expanded views of the tip areas are given in inverted contrast. The microtubule network was co-visualized through expression of mCherry-a-tubulin. While most kinesin-4 proteins could be detected in the cytosol (as indicated with an asterisk), only Kin4-la labelling produced striped, overlap-like staining throughout the cell (arrowheads). Three other kinesin-4s gave punctate labelling in the tip of the cell (arrows), with Kin4-Ila additionally showing decoration of the plasma membrane at the subapex (indicated with ' $s$ '). The phylogenetic relationship between the kinesin-4s is depicted by a tree inferred using the maximum likelihood method following the nomenclature of Shen et al. (2012). Bootstrap values (derived from 500 replicates) indicate the percentage of trees in which the associated taxa clustered together. Horizontal branch lengths represent the number of amino acid substitutions per site. Scale bars, $5 \mu \mathrm{m}$. 
A

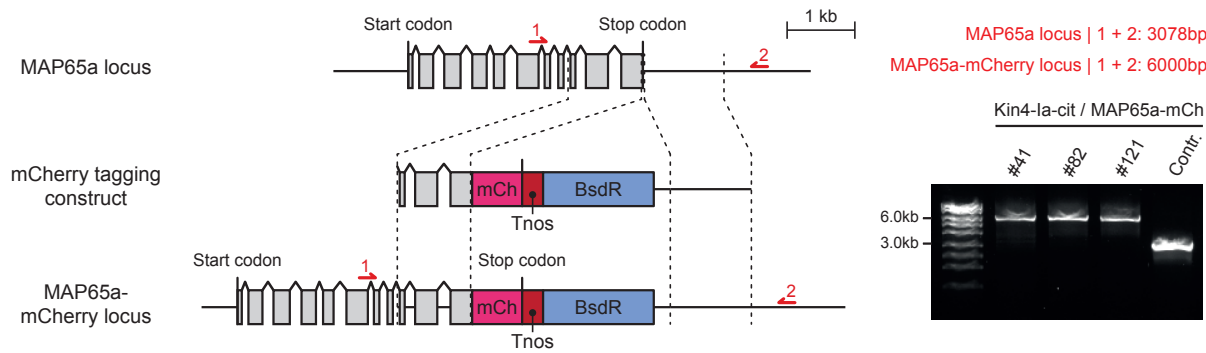

B

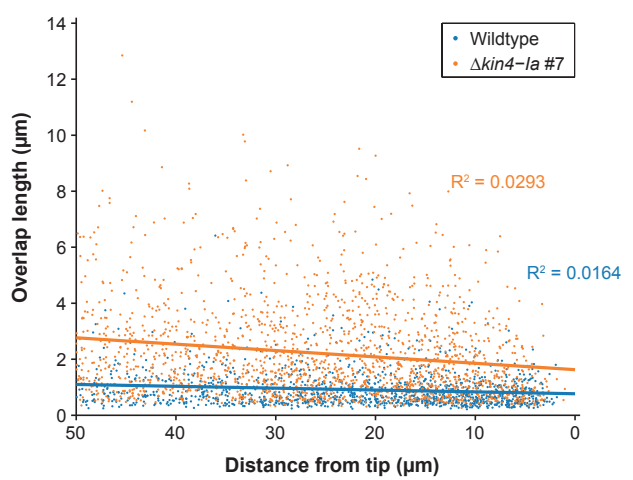

C

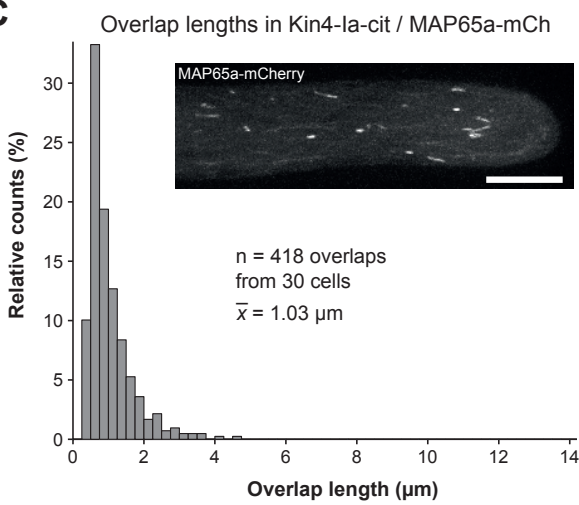

Figure S2 - Generation and validation of a MAP65a / Kin4-la co-localization strain and overlap length dependency on distance to the tip. (A) Schematic representation of the genomic locus containing the MAP65a encoding gene with its intron-exon structure (gray boxes) and the construct used for C-terminal mCherry tagging via homologous recombination (dashed lines). In MAP65a-labelled lines, the original stop codon is replaced with a fragment containing the mCherry encoding sequence (pink box), the nopaline synthase terminator (red box) and a cassette conferring blasticidin resistance (blue box). Red arrows denote primer binding sites used for confirmation of the obtained lines by PCR. The product obtained after PCR reaction and its predicted size are given on the right. The numbers of three independently obtained transformants are given above the gel images. (B) Scatterplot showing the correlation between overlap length and distance to the cell tip (as visualized with MAP65a-citrine expression). Individual measurements of the length of an overlap are plotted against the distance of its centre to the cell tip for $n=1238$ overlaps (wild-type) and $n=1781$ overlaps ( $\Delta$ kin4-la line \#7). Both in wild-type and the $\Delta$ kin4-la mutant simple linear regression analysis revealed an extremely weak correlation. (C) Histogram showing the length distribution of the indicated amount of MAP65a-mCherry labelled overlaps from the Kin4-la-citrine / MAP65a-mCherry co-localization line. An example of the MAP65a-mCherry signal in a caulonemal tip cell is depicted in the inset (z-projection of 20 planes spaced $0.4 \mu \mathrm{m}$ apart. Scale bar, 10 $\mu \mathrm{m})$. Mean overlap length was both significantly smaller than in the $\Delta$ kin4-la lines and significantly higher than in de MAP65a-citrine control line using a Kruskal-Wallis test with pairwise comparisons $(P<0.001)$. (Compare with Figure $2 A-B)$ 
5 
A

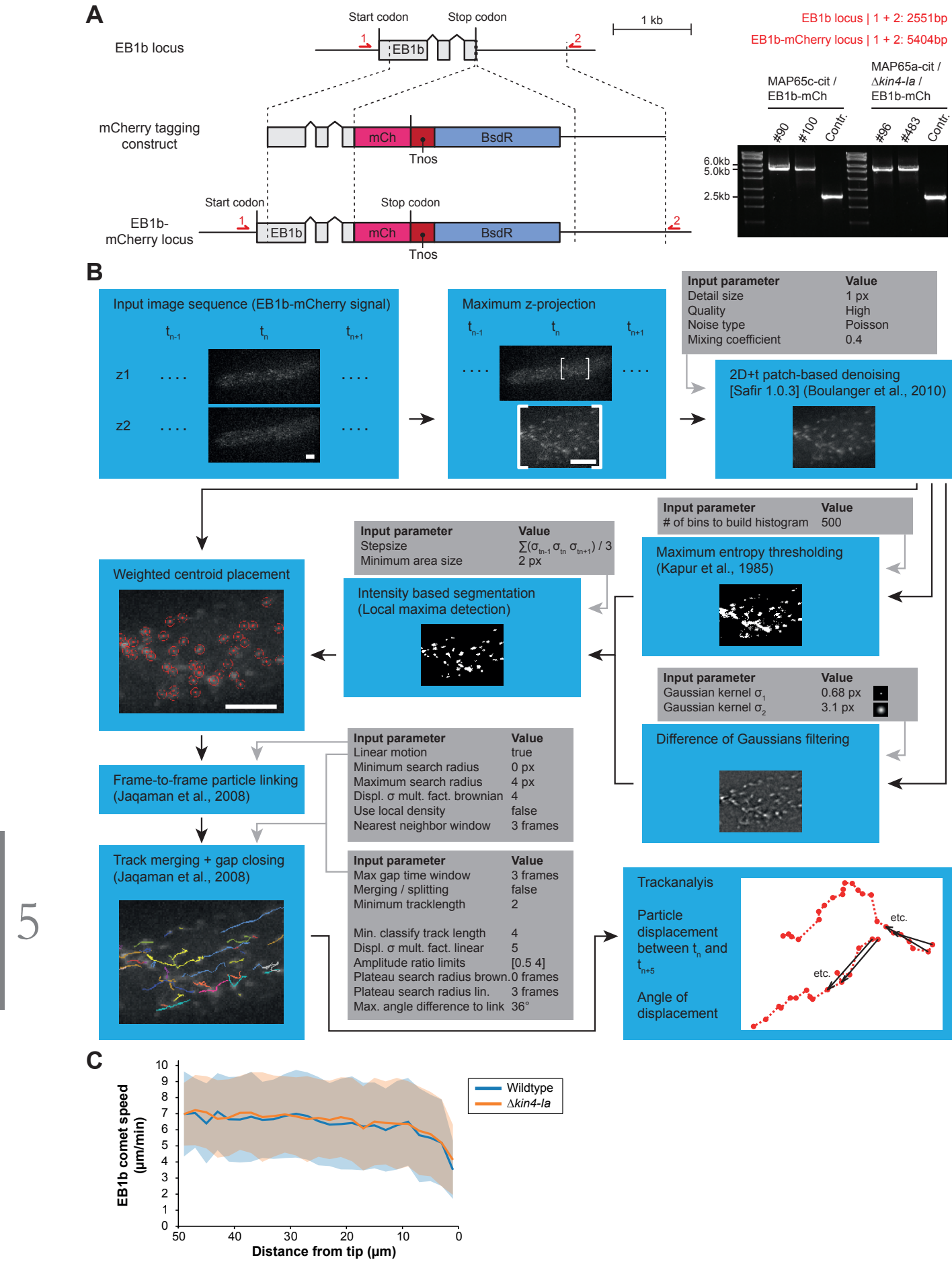

EB1b locus | 1 + 2: 2551 bp

Figure S3 - Generation of an EB1b-mCherry marker strain and computational tracking of EB1b-labelled comets. (A) Schematic representation of the genomic locus containing the EB1b

(legend continued on next page) 
encoding gene with its intron-exon structure (gray boxes) and the construct used for C-terminal $m$ Cherry tagging via homologous recombination (dashed lines). In EB1b-labelled lines, the original stop codon is replaced with a fragment containing the mCherry encoding sequence (pink box), the nopaline synthase terminator (red box) and a cassette conferring blasticidin resistance (blue box). Red arrows denote primer binding sites used for confirmation of the obtained lines by PCR. The product obtained after PCR reaction and its predicted size are given on the right. The numbers of two independent transformants are given above the gel images. (B) Schematic depiction of the workflow for EB1b-comet tracking including images illustrating the steps used. For a full description of the workflow refer to the materials and methods section. In brief, an input image sequence acquired at 2 z-planes was used to generate a maximum z-projection. After reducing image noise, an intensity threshold was determined using the maximum entropy method and the image data was filtered with a Difference of Gaussians (DoG) kernel. Thresholded areas were further segmented by detecting the local maxima within them using the DoG-filtered data. Only regions bigger than a defined minimum size were retained. An intensity-weighted centroid was then determined for each accepted particle (red circles). Subsequently, the particles were linked frame-to-frame using a published method (Jaqaman et al. 2008) generating short, incomplete tracks. These tracks were then combined into the final tracks using a second global optimization step (lines of varying colour; (Jaqaman et al. 2008)). Within each final track, velocity and angle of particle displacement were determined between frame $n$ and frame $n+5$ (referred to as track segments; black arrows). The bracketed area is shown in detail for all image processing steps for clarity. Scale bars, $5 \mu \mathrm{m}$. (C) Average EB1b-comet speed plotted as a function of the distance between the tip and the centre of the measured track segment for wild-type and a $\Delta k i n 4-l a$ mutant background. Across both genotypes velocities were highly similar along the entire length of the cell. Interestingly, both show a mild decrease in comet velocity near the apex. Distances to the tip were binned in $2 \mu \mathrm{m}$ bins before averaging. The shaded areas indicate standard deviation. Wild-type data from 16567 track segments from 3244 tracks from 21 cells and $\Delta$ kin4-la mutant data from 14708 track segments from 2708 tracks from 15 cells. 
A Wild-type
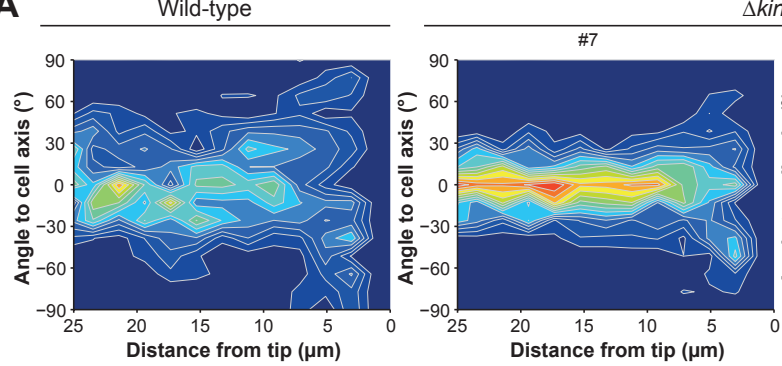

skin4-la
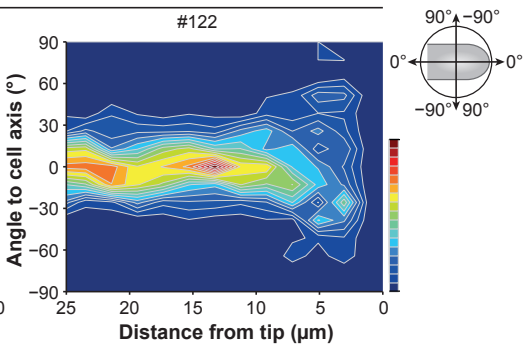

B

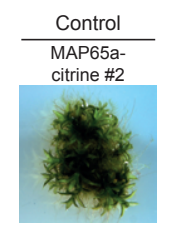

C

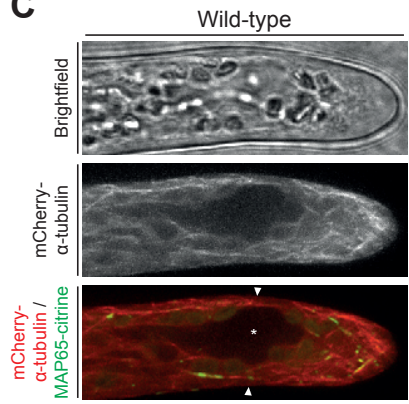

mCherry- $\alpha$-tubulin / MAP65a-citrine

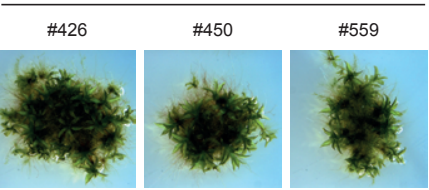

$\Delta$ kin4-la

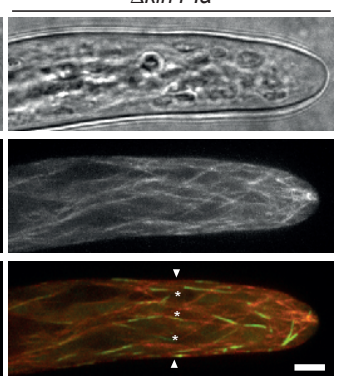

Control

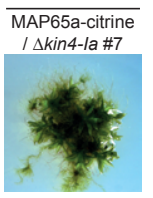

D

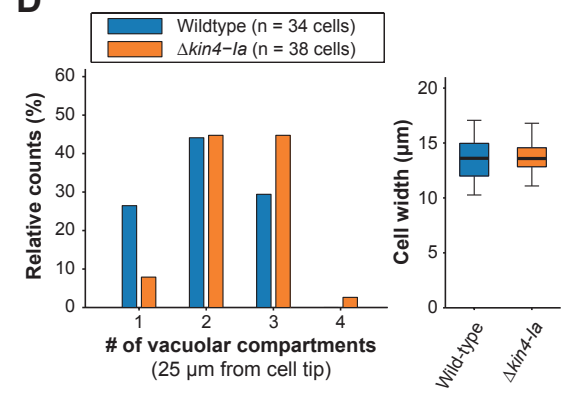

mCherry-a-tubulin / MAP65a-citrine / $\Delta$ kin4-la

\#14 \#85 \#22
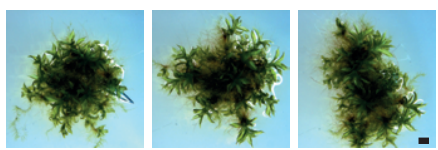

Figure S4 - Colony appearance of $m$ Cherry- $\alpha$-tubulin expressing strains and indicators of microtubule network hyper-parallelization. (A) Contour plots with colour map showing the angle distribution of MAP65a-citrine labelled overlaps as a function of distance to the cell tip for wild-type and two $\Delta$ kin4-la mutant lines. Upon Kin4-la removal overlaps show closer alignment with the cell axis relative to the wild-type situation. (B) Colony appearance of moss lines with an introduced $\mathrm{mCherry- \alpha -tubulin} \mathrm{expression} \mathrm{construct} \mathrm{in} \mathrm{a} \mathrm{wild-type} \mathrm{and} \Delta$ kin4-la genetic background. For each moss line three independent transformants are shown. Introduction of the expression construct did not lead to altered colony size or morphology which would indicate growth defects. Plants were photographed after 5 weeks of growth on BCD agar plates. Scale bar, $1 \mathrm{~mm}$. (C) Topology of the microtubule network in the median plane of a wild-type and $\Delta k i n 4-l a$ caulonemal tip cell visualized through expression of mCherry- $\alpha$-tubulin. A brightfield image of the imaged cell is shown above and an overlay with the corresponding MAP65a-citrine signal is given below. Here, the arrowheads illustrate the section transverse to the length axis, $25 \mu \mathrm{m}$ from the tip used for counting the degree of vacuolar partitioning as presented in C. Vacuole compartments separated by transvacuolar strands are indicated with an asterisk. Images of the fluorescent probes are maximum z-projections of 9 planes spaced $0.27 \mu \mathrm{m}$ apart. Scale bar, $5 \mu \mathrm{m}$. (D) Histogram showing quantification of the amount of vacuole compartments measured $25 \mu \mathrm{m}$ from the cell tip that were visible in the median plane for the indicated amount of wild-type and $\Delta k$ in $4-l a$ caulonemal tip cells. The vacuolar compartments in $\Delta k i n 4-l a$ showed a higher degree of fragmentation parallel the length axis of the cell than recorded for wild-type ( $P=0.033$ in a Mann-Whitney $U$ test). The boxplots presented on the right show that the average cell width recorded at the location where vacuole measurements were taken was similar for wild-type and $\Delta k$ in 4 -la cells ( $P>0.05$ in an unpaired Student's $t$-test). The whiskers indicate minimum / maximum observed values. 


\section{References}

Anderhag, P., Hepler, P.K. \& Lazzaro, M.D., 2000. Microtubules and microfilaments are both responsible for pollen tube elongation in the conifer Picea abies (Norway spruce). Protoplasma, 214(3-4), pp.141-157.

Bibikova, T.N., Blancaflor, E.B. \& Gilroy, S., 1999. Microtubules regulate tip growth and orientation in root hairs of Arabidopsis thaliana. Plant Journal, 17(6), pp.657-665.

Bieling, P., Telley, I. \& Surrey, T., 2010. A minimal midzone protein module controls formation and length of antiparallel microtubule overlaps. Cell, 142(3), pp.420-432.

Boulanger, J. et al., 2010. Patch-based nonlocal functional for denoising fluorescence microscopy image sequences. IEEE transactions on medical imaging, 29(2), pp.442-454.

Bratman, S. V. \& Chang, F., 2007. Stabilization of overlapping microtubules by fission yeast CLASP. Developmental Cell, 13(6), pp.812-827.

Bringmann, H. et al., 2004. A kinesin-like motor inhibits microtubule dynamic instability. Science, 303(5663), pp.1519-1522.

Burk, D.H., Zhong, R. \& Ye, Z.H., 2007. The katanin microtubule severing protein in plants. Journal of Integrative Plant Biology, 49(8), pp.1174-1182.

Chan, J. et al., 1999. The 65-kDa carrot microtubule-associated protein forms regularly arranged filamentous cross-bridges between microtubules. Proceedings of the National Academy of Sciences of the United States of America, 96(26), pp.14931-14936.

Cole, R.A. \& Fowler, J.E., 2006. Polarized growth: maintaining focus on the tip. Current Opinion in Plant Biology, 9(6), pp.579-588.

Cove, D.J. et al., 1978. Genetic and physiological studies of the effect of light on the development of the moss, Phycomitrella patens. Photochemistry and Photobiology, 27(2), pp.249-254.

Dixit, R., Chang, E. \& Cyr, R., 2006. Establishment of polarity during organization of the acentrosomal plant cortical microtubule array. Molecular Biology of the Cell, 17(3), pp.1298-1305.

Dixit, R. \& Cyr, R., 2004. Encounters between dynamic cortical microtubules promote ordering of the cortical array through angle-dependent modifications of microtubule behavior. The Plant Cell, 16(12), pp.3274-3284.

Domozych, D., Fujimoto, C. \& LaRue, T., 2013. Polar expansion dynamics in the plant kingdom: A diverse and multifunctional journey on the path to pollen tubes. Plants, 2(1), pp.148-173.

Doonan, J., Cove, D.J. \& Lloyd, C., 1988. Microtubules and microfilaments in tip growth: evidence that microtubules impose polarity on protonemal growth in Physcomitrella patens. Journal of Cell Science, 89, pp.533-540.

Duellberg, C. et al., 2013. End-binding proteins and Ase1/PRC1 define local functionality of structurally distinct parts of the microtubule cytoskeleton. Trends in Cell Biology, 23(2), pp.54-63.

Eng, R.C. \& Wasteneys, G.O., 2014. The microtubule plus-end tracking protein ARMADILLO-REPEAT KINESIN1 promotes microtubule catastrophe in Arabidopsis. The Plant Cell, 26(8), pp.3372-3386.

Fink, G. et al., 2009. The mitotic kinesin-14 Ncd drives directional microtubule-microtubule sliding. Nature Cell Biology, 11(6), pp.717-723.

Gaillard, J. et al., 2008. Two microtubule-associated proteins of Arabidopsis MAP65s promote antiparallel microtubule bundling. Molecular Biology of the Cell, 19(10), pp.4534-4544.

Gilroy, S. \& Jones, D.L., 2000. Through form to function: Root hair development and nutrient uptake. Trends in Plant Science, 5(2), pp.56-60.

Hiwatashi, Y., Sato, Y. \& Doonan, J.H., 2014. Kinesins have a dual function in organizing microtubules during both tip growth and cytokinesis in Physcomitrella patens. The Plant Cell, 26(3), pp.1256-1266.

Ishitsuka, Y. et al., 2015. Superresolution microscopy reveals a dynamic picture of cell polarity maintenance during directional growth. Science Advances, 1(10), e1500947.

Jaqaman, K. et al., 2008. Robust single-particle tracking in live-cell time-lapse sequences. Nature Methods, 5(8), pp.695-702.

Jenkins, G.I., Courtice, G.R. \& Cove, D.J., 1986. Gravitropic responses of wild-type and mutant strains of the moss Physcomitrella patens. Plant, Cell \& Environment, 9(8), pp.637-644.

Justus, C.D. et al., 2004. Microtubules and microfilaments coordinate to direct a fountain streaming pattern in elongating conifer pollen tube tips. Planta, 219(1), pp.103-109.

Kapur, J.N., Sahoo, P.K. \& Wong, A.K.C., 1985. A new method for gray-level picture thresholding using the entropy of the histogram. Computer Vision, Graphics, and Image Processing, 29(3), pp.273-285. 
de Keijzer, J. et al., 2017. Shortening of microtubule overlap regions defines membrane delivery sites during plant cytokinesis. Current Biology, 27(4), pp.514-520.

de Keijzer, J., Mulder, B.M. \& Janson, M.E., 2014. Microtubule networks for plant cell division. Systems and Synthetic Biology, 8(3), pp.187-194.

Ketelaar, T., 2013. The actin cytoskeleton in root hairs: All is fine at the tip. Current Opinion in Plant Biology, 16(6), pp.749-756.

Ketelaar, T., 2003. Unstable F-actin specifies the area and microtubules direction of cell expansion in Arabidopsis root hairs. The Plant Cell, 15(1), pp.285-292.

Kosetsu, K. et al., 2013. MICROTUBULE-ASSOCIATED PROTEIN65 is essential for maintenance of phragmoplast bipolarity and formation of the cell plate in Physcomitrella patens. The Plant Cell, 25(11), pp.4479-4492.

Kost, B., 2008. Spatial control of Rho (Rac-Rop) signaling in tip-growing plant cells. Trends in Cell Biology, 18(3), pp.119-127.

Lee, Y.J. \& Yang, Z., 2008. Tip growth: signaling in the apical dome. Current Opinion in Plant Biology, 11(6), pp.662-671.

Matov, A. et al., 2010. Analysis of microtubule dynamic instability using a plus-end growth marker. Nature Methods, 7(9), pp.761-768.

Miki, T. et al., 2014. Endogenous localizome identifies 43 mitotic kinesins in a plant cell. Proceedings of the National Academy of Sciences of the United States of America, 111(11), pp.1053-1061.

Nakaoka, Y. et al., 2015. Cytoplasmic nucleation and atypical branching nucleation generate endoplasmic microtubules in Physcomitrella patens. The Plant Cell, 27(1), pp.228-242.

Nishiyama, T. et al., 2000. Tagged mutagenesis and gene-trap in the moss, Physcomitrella patens by shuttle mutagenesis. DNA Research, 7(1), pp.9-17.

Oda, Y. et al., 2009. Microtubules regulate dynamic organization of vacuoles in Physcomitrella patens. Plant \& Cell Physiology, 50(4), pp.855-868.

Püspöki, Z. et al., 2016. Transforms and operators for directional bioimage analysis: A survey. In Advances in Anatomy, Embryology and Cell Biology. pp. 69-93.

Rounds, C.M. \& Bezanilla, M., 2013. Growth mechanisms in tip-growing plant cells. Annual Review of Plant Biology, 64(1), pp.243-265.

Shaw, S.L. \& Lucas, J., 2011. Intrabundle microtubule dynamics in the Arabidopsis cortical array. Cytoskeleton, 68(1), pp.56-67.

Shen, Z. et al., 2012. Phylogenetic analysis of the kinesin superfamily from Physcomitrella. Frontiers in Plant Science, 3, p.230.

Sieberer, B.J. et al., 2005. Microtubules guide root hair tip growth. New Phytologist, 167(3), pp.711-719.

Smertenko, A.P. et al., 2004. The Arabidopsis microtubule-associated protein AtMAP65-1: Molecular analysis of its microtubule bundling activity. The Plant Cell, 16(8), pp.2035-2047.

Takeda, S. et al., 2008. Local positive feedback regulation determines cell shape in root hair cells. Science, 319(5867), pp.1241-1244.

Tulin, A. et al., 2012. Single-molecule analysis of the microtubule cross-linking protein MAP65-1 reveals a molecular mechanism for contact-angle-dependent microtubule bundling. Biophysical Journal, 102(4), pp.802-809.

Vidali, L. et al., 2009. Lifeact-mEGFP reveals a dynamic apical F-actin network in tip growing plant cells. PloS one, 4(5), e5744.

Wasteneys, G.O. \& Ambrose, J.C., 2009. Spatial organization of plant cortical microtubules: close encounters of the 2D kind. Trends in Cell Biology, 19(2), pp.62-71.

Yang, G. et al., 2007. A mutation in MRH2 kinesin enhances the root hair tip growth defect caused by constitutively activated ROP2 small GTPase in Arabidopsis. PLoS one, 2(10), e1074.

Zhang, B., Zerubia, J. \& Olivo-Marin, J.-C., 2007. Gaussian approximations of fluorescence microscope point-spread function models. Applied Optics, 46(10), pp.1819-1829.

Zhu, L. et al., 2013. MAP18 regulates the direction of pollen tube growth in Arabidopsis by modulating F-actin organization. The Plant Cell, 25(3), pp.851-867. 
-88.

$\because 8 \% 8$

Chapter 6 - General discussion: shaping $\therefore$ microtubule networks for plant cell $\because$ division and expansion

\section{Jeroen de Keijzer}

Laboratory of Cell Biology, Wageningen

University, Droevendaalsesteeg 1, 6708 PB

Wageningen, The Netherlands

"A rolling stone gathers no moss"

- Publilius Syrus, ca 50 bc
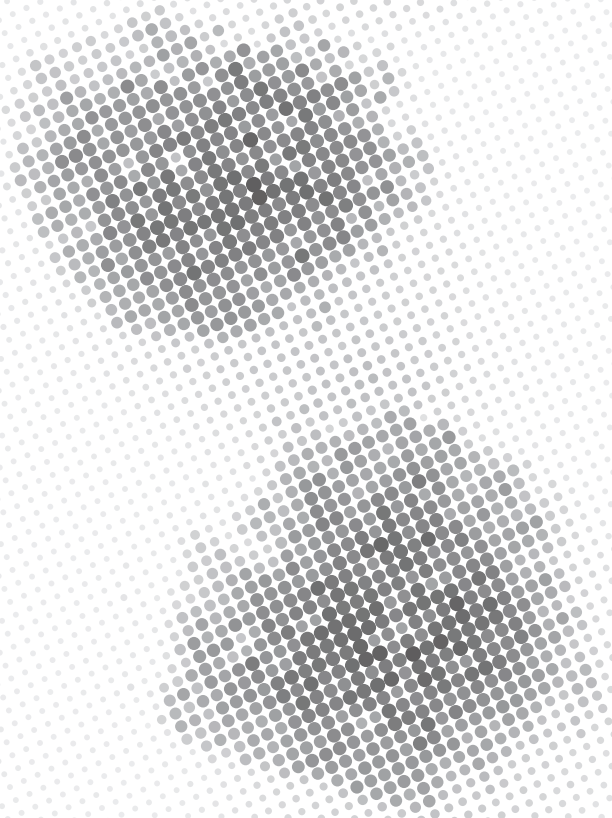
Microtubules are dynamic polymers assembled into a vast array of structured networks for various vital cellular functions. In this thesis I focused on the role of antiparallel microtubule overlaps in shaping microtubule networks that drive plant cell division and apical cell expansion. This led to the discovery that overlaps act as a scaffold for spatial orchestration of cytokinesis. Furthermore, we have shown that the interphase microtubule network of tip growing cells forms an important node in the relay of environmental signals to plant morphogenesis. In this chapter I will discuss the topics where the key findings presented in this thesis led to differences in perspective with other studies, can be expanded to other fields in interesting ways, give rise to new questions for future investigation, and generate new understanding. Inherent to this approach, the discussed topics will be presented in several separate sections. Firstly, I will give an evolutionary view on the link between phragmoplast overlaps and cell plate assembly. Then, I will briefly compare the design principles of the spindle and phragmoplast arrays and discuss that of the interphase network. Finally, a brief perspective on the broader relevance of the findings will be given.

\subsection{Phragmoplast function in an evolutionary perspective}

Cell plate-assisted execution of cytokinesis via formation of a phragmoplast is an evolutionary innovation encountered in land plants and some related algae (Buschmann \& Zachgo 2016). While dissimilar at first glance, several parallels between the phragmoplast microtubule array in plants and the central spindle directing cytokinetic processes in animal cells have been drawn (Euteneuer \& Mcintosh 1980; Otegui et al. 2005). In fact, the first study describing the animal midbody (the central spindle in late cytokinesis) refers to it as a 'possible cell-plate equivalent' (Flemming 1891). Moreover, functional homology between components organizing the plant and animal cytokinetic microtubule networks, such as the recurrent use of Ase1p/PRC1/ MAP65 family microtubule bundlers, became increasingly evident (Otegui et al. 2005). However, any notion to what degree (if at all) the relatedness of the two systems can be attributed to inheritance of cellular machinery regulating cytokinesis in the last common ancestor is currently lacking. In general, by exposing if there are conserved elements involved in the temporal and spatial regulation of eukaryotic cytokinesis, the origin and roles of lineage-specific adaptations can be better understood.

\section{Conservation of an overlap-associated kinesin-4 in eukaryotes}

Our results on the role of kinesin-4s in regulating overlap geometry during plant cell division described in chapter 2 begin to shine light on possible conservation of mitotic microtubule activities. The localization and function of kinesin4-Ic in moss provides strong indications that this motor is a true orthologue of a class of kinesin-4 molecules found in animals. Like Xklp1 in Xenopus, KIF4 in humans and KLP3A in Drosophila, moss kinesin4-Ic localizes to chromosome arms during metaphase to rapidly transition to the zone of overlapping microtubules at the onset of anaphase, where it serves to stabilize the late-mitotic microtubule array (Vernos et al. 1995; Williams et al. 1995; Kurasawa et al. 2004; de Keijzer et al. 2017). It is highly unlikely that this distinct set of properties combined in one protein could independently arise twice in eukaryotic evolution, thus supporting conservation of functions already present in a similar molecule of the last common ancestor of plants and animals. Also, the finding that the moss kinesin4-Ia/MAP65 system described in chapter 5 shares several molecular properties (i.e. recruitment and motility characteristics) with its Xenopus counterpart, reinforces the idea that kinesin-4 functioning 
during cell division is an ancestral trait. Plants and animals belong to two different eukaryotic supergroups (Plantae and Opisthokonta respectively), whose precise taxonomical relationship is not yet precisely resolved (Williams 2014; Butterfield 2015). Nevertheless, they are believed to have diverged (along with the other supergroups) soon after the arrival of the pan-eukaryotic ancestor approximately 1.6 billion years ago (Eme et al. 2014; Butterfield 2015; Knoll \& Nowak 2017). This implies that the different functional aspects of kinesin- 4 were present at that time, therefore hinting at an ancient role in the mitotic phase. The conservation of kinesin- 4 functions means that they could be encountered in many other eukaryotic organisms alive today, especially these belonging to the Plantae and Opisthokonta supergroups.

The finding of chapter 2 that overlap shortening is essential for confined cell plate membrane accumulation, gives an intriguing opening to speculate on the transition within the plant kingdom of a cleavage mode of cell division to a cell-plate assisted mode. As mentioned above, the phragmoplast-assisted mode of cell division is an evolutionary innovation that occurred in the ancestor of land plants and several related streptophyte algae (Buschmann \& Zachgo 2016) (See also figure 4 of chapter 1). The algal sister-lineages of this clade have a cleavage mode of cell division, possibly retained from the earliest, single-celled ancestral plant (Pickett-Heaps 1975; Pickett-heaps et al. 1999; Buschmann \& Zachgo 2016). As stated above, a cross-linker kinesin-4 module for overlap length control has been conserved in two different eukaryotic supergroups, suggesting it proved valuable in various cellular contexts throughout the course of evolution. Our results point out that during phragmoplast-assisted cell division, length control of overlaps is an important aspect of microtubule network lay-out for shaping the division plane. A late-mitotic microtubule array resembling the animal central spindle is reported to form in streptophyte algae that use cell separation by cleavage and are sister to the clade that acquired the phragmoplast-assisted mode (Katsaros et al. 2011). Unfortunately, in these organisms, the precise network geometry and presence of overlaps is not well-documented yet, although this could provide insight into how the phragmoplast developed from such a central spindle-like array. In a microtubule constellation that has to dynamically reorganize to add new material to a free-floating, flat division plane the importance of strict spatial control of overlaps has likely increased with respect to the ancestral late-mitotic microtubule array supporting a cleavage mode of cell division. The evolutionary conservation of kinesin-4 precludes a large role in this transition, making other, possibly newly evolved factors relevant for putting further constraints on overlap length and relative positioning of overlaps with respect to each other. Interestingly, in select algae a hybrid mechanism of cell division using both a cleavage furrow and a phragmoplast is encountered (Fowke \& Pickett-Heaps 1969; Pickett-heaps et al. 1999). This method most likely represents a partial reversal to the ancestral cleavage mode after the phragmoplast mode was acquired (Buschmann \& Zachgo 2016). Comparative studies among such algae and representatives of the phragmoplast-assisted mode and cleavage mode could bring to light what cellular innovations concerning overlap control are required for phragmoplast functioning. In a similar fashion, the innovations that led to the ability to fuse vesicles into a new membrane compartment in absence of a pre-existing target membrane could be investigated (further discussed in 6.2).

\section{Making ends meet: overlaps in flowering plants}

How does the finding that overlap length regulation intimately ties in with the cell plate assembly process in a moss compare to what is known about the role of overlaps in flowering plants? Investigations of the past 20 years, performed mainly in Arabidopsis, have reached conflicting 
conclusions on the presence and role of microtubule overlaps in flowering plants. Detailed electron tomography studies of Arabidopsis meristematic cells found that very few phragmoplast microtubules extend across the division plane, a prerequisite for overlap formation (SeguíSimarro et al. 2004; Austin et al. 2005). These authors instead describe 'head-on' engagement of phragmoplast microtubule plus ends from opposite poles with the so-called cell plate assembly matrix, a thin, ribosome-free area surrounding the cell plate membrane (Austin et al. 2005). The authors postulate that this association stabilizes the microtubule plus ends. On the other hand, MAP65-3, one of the MAP65-family microtubule crosslinking proteins of Arabidopsis, localizes to the division plane, suggesting the formation of overlaps. Moreover, in MAP653 mutant cells engagement of phragmoplast microtubules from opposite poles is hampered, suggesting overlaps are required for phragmoplast stability. (Müller et al. 2004; Ho et al. 2011). This finding, together with electron-microscopical observations that do show interdigitating microtubules in the Arabidopsis phragmoplast, makes a case that there is functional microtubule overlap formation during cytokinesis in flowering plants (Ho et al. 2011). Further investigation of other MAP65-family proteins in Arabidopsis has shown that they function in addition to MAP65-3 (Sasabe et al. 2011; Ho et al. 2012; Li et al. 2017). Meanwhile, factors that govern the length of phragmoplast overlaps in higher plants are still unknown. Given the conservation of kinesin-4 function among animals and moss, a motor belonging to this class of kinesins could be a prime candidate in higher plants. However, there is no clear indication that any of the three kinesin-4 motors encoded in the Arabidopsis genome are involved in cytokinesis (Kong et al. 2015). Thus, while there is ample reason to believe that overlaps do form in the Arabidopsis phragmoplast and play a role in its stability, there could be kinesin-4-independent mechanisms of length control, or, more general, the contribution of overlap length in delimiting the site of cell plate assembly has become less outspoken in Arabidopsis as it is in Physcomitrella.

If there would indeed be a diminished participation of microtubule overlaps in defining the site of cell plate formation in flowering plants, what phragmoplast properties could then explain this difference with moss? Some of the observations in the moss kinesin- 4 mutants already point to possibilities. We observed that membrane material, as well as exocyst subunit Sec6, did not decorate the entire length of over-extended overlaps in absence of kinesin-4 (Chapter $2 \& 3$ ). Instead, they both concentrated at the centre of overlaps, a phenomenon that became more noticeable as cytokinesis progressed. Thus, secondary processes contribute to the spatial confinement of membrane. This highlights that the overlap-defined zone appears to be subcompartmentalized by other processes, narrowing the distribution of cell plate membrane to only the centre of overlaps. Interestingly, the MAP65 signal within over-elongated overlaps peaked at the same location, indicating a potential link between membrane-associated factors and MAP65. Since interactions of MAP65 with membrane-associated factors (physical and/or chemical) are expected to decrease its turnover rate, we photo-bleached citrine-tagged MAP65 in kinesin-4 double mutant cells. We found that MAP65 turnover was slow near the centre of overlaps where membrane material was accumulating, whilst it was faster near overlap ends that were devoid of membrane (Figure 1A-B). This is indicative of a local interaction, but one needs to assume that recovery of MAP65 was not limited locally by membranous material slowing down cytosolic diffusion. The cell plate membrane structures that are delimited by secondary processes, thus appear to provide feedback to MAP65. This highlights that there is not only a flow of positional information from overlaps to the forming cell plate, but also vice versa. It should be noted however that these observations stem from an artificial mutant situation and could therefore operate differently under wild-type conditions. The dimensions of the overlaps found 
in wild-type phragmoplast are close to or below the resolution limit of the optical microscope, currently precluding similar experiments. Nonetheless, I expect that the two principles discussed here (additional confinement of the cell plate through overlap-independent, intrinsic factors and feedback between the forming cell plate and overlaps), can contribute to understanding why overlaps appear to have a diminished role in Arabidopsis compared to moss.

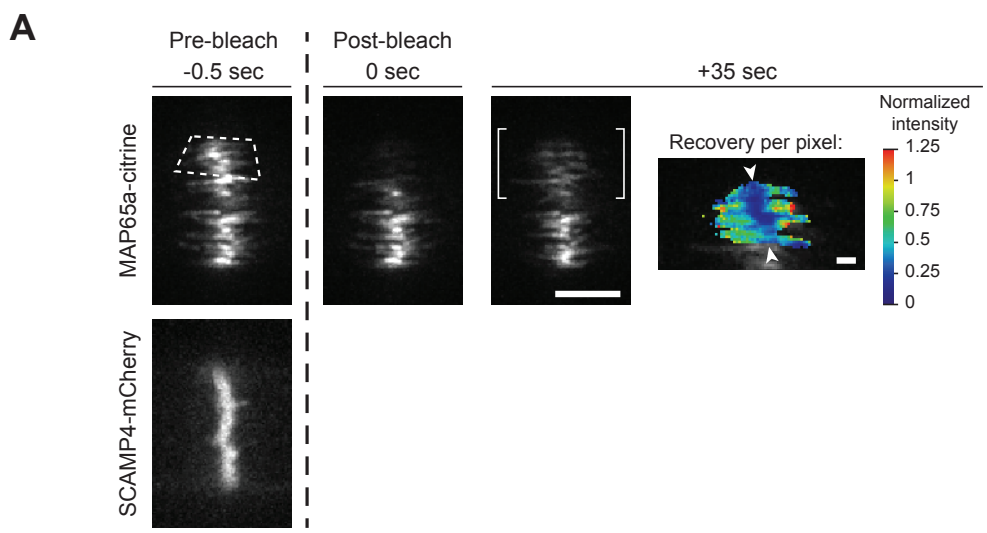

B
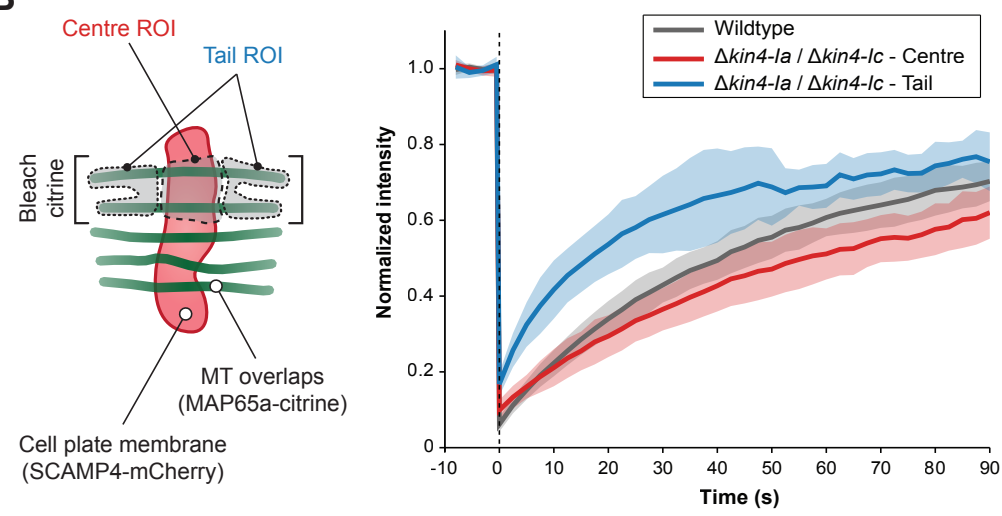

Cell plate membrane (SCAMP4-mCherry)

Figure 1 - Characterization of microtubule overlap - cell plate membrane interaction. (A) Turnover of MAP65 in the phragmoplast midplane in $\Delta$ kin4la / $\triangle$ kin4-Ic / SCAMP4-mCh cells analysed using fluorescence recovery after photobleaching. The dashed line indicates the bleached region. The degree of recovery was calculated on a per pixel basis. Arrowheads indicate the central region of the overlaps that corresponds to the region of the maturing cell plate demonstrated by strong labelling with SCAMP4-mCherry. Here, MAP65a-citrine turnover is slow compared to the protruding regions of microtubule overlap that have little associated membrane. Images are maximum z-projections of 3 confocal planes spaced $0.5 \mu \mathrm{m}$ apart. The left and right scalebar indicate $5 \mu \mathrm{m}$ and $1 \mu \mathrm{m}$ respectively. (B) Quantification of MAP65a-citrine fluorescence recovery in the central cell plate associated region ("centre") compared to the regions of overlap protruding from the cell plate ("tail") in the $\Delta$ kin4-la / $\Delta$ kin4-Ic mutant (n $=8$ cells). The schematic depicts the regions of interest (ROIs) used for fluorescence recovery measurements. The rate of recovery in the central region of this mutant closely resembled that of MAP65a in wild-type cells $(n=15$ cells) that lack overlap "tails". Curves show average and standard deviation for the indicated number of cells. 
Our findings in moss can be unified with existing data from flowering plants in a scenario where overlaps position the early membrane accumulation and fusion processes, after which other, overlap-independent remodelling processes become increasingly important for shaping the cell plate. In flowering plants, the latter processes may have taken up a bigger role at the expense of overlap-bound processes, or, alternatively, microtubules that do not form overlaps contribute to positioning of the cell plate, perhaps via their plus ends embedded in the cell plate assembly matrix (Austin et al. 2005). Several lines of future experimentation are required to corroborate these scenarios. Firstly, molecular players acting as cell plate membrane remodellers have been identified including dynamins and a SH3 domain-containing protein (Hong et al. 2003; Mcmichael \& Bednarek 2013; Ahn et al. 2017). This opens up avenues to look how intrinsic cell-plate membrane remodelling factors cooperate with overlap-associated processes (e.g. by making kinesin-4 mutants with additionally these factors knocked-out). How membrane fusion and remodelling processes, but also phragmoplast microtubules, are connected to the cell plate assembly matrix is currently not understood. To elucidate the functional aspects of this structure it will be crucial to overcome the current lack of definition of the cell plate assembly matrix in molecular regard. This makes it imperative to learn more about the fundamentals of this structure in the coming years. Finally, the proposed absence of a cytokinetic kinesin- 4 in Arabidopsis (Kong et al. 2015), raises the question at what point in the course of land plant evolution this factor (and by extension perhaps the tight control of overlap length) was lost. Investigations into representatives of other major plant lineages could address this question. The recent identification of a kinesin-4 with mitotic function in the fern Marsilea vestita certainly proposes to be a good starting point (Tomei \& Wolniak 2016).

All in all, Physcomitrella is a highly interesting organism for cytokinesis research. It shares conserved components with animal cytokinesis, while featuring a full-fledged mode of cell-plate and phragmoplast-assisted cell separation. This combination makes it an eminently effective instrument to understand how ancient eukaryotic principles of cell division and innovations within the green kingdom have intersected and led to the emergence of an intriguing and flexible method of separating two daughter cells.

\subsection{Linking the membrane-based partitioning system to a microtubule structure}

In this thesis we expose important new features of antiparallel microtubule overlaps during plant cell division. We show that overlaps are not only a structural element, but used to construct a localized biochemical environment for the positioning of membrane material and vesicle tethering factors used to build the division plane. Such a scaffolding function of overlaps has been described for the overlaps of the animal central spindle (The late-mitotic microtubule network regulating cytokinesis in animals) (Mishima et al. 2002; Zhu et al. 2006; Glotzer 2009; Lee et al. 2012; Duellberg et al. 2013). However, here, except during the final abscission step (Lekomtsev et al. 2012), they do not make direct contact with the division machinery (i.e. the contractile ring) in contrast to the situation in the phragmoplast. The intimate association between microtubule overlaps and membrane-associated processes are probably mediated through a range of control mechanisms. In this part I will discuss how some of the known mechanisms regulating cell plate membrane assembly and fusion could be linked to microtubule overlaps.

It has been established that secretory membrane trafficking pathways are required for cell-plate 
biogenesis (Mcmichael \& Bednarek 2013; Boruc \& Van Damme 2015). Generally membrane trafficking involves the budding of a vesicle from one membranous compartment and the subsequent fusion of the vesicle to a compartment with a different identity. However, during cell plate biogenesis two problems arise that require special adaptations to the canonical trafficking mechanism. i) No pre-existing target membrane exists, thus homotypic vesicle fusion is required. ii) Vesicle fusion should not occur prematurely or in a wrong location.

A string of successive studies in Arabidopsis have led to the emergence of a detailed description of the mechanism promoting the fusion of identical vesicles during cell plate formation, while preventing their premature fusion during vesicle formation and trafficking (Lauber et al. 1997; Waizenegger et al. 2000; Park et al. 2012; Richter et al. 2014; Jürgens et al. 2015; Karnahl et al. 2017). Cytokinesis specific Soluble NSF Attachment Protein Receptor (SNARE) complexes play a central role in this mechanism. Early in the membrane trafficking pathway the four parts of the SNARE complex used for cell plate membrane fusion are already assembled together in a lowenergy configuration, a state that would normally only arise after a successful fusion event of a vesicle (containing 1 SNARE component) with its target membrane (containing 3 components) (Karnahl et al. 2017). Vesicles containing this low-energy SNARE complex can thus be safely trafficked through different endomembrane compartments without a chance of spurious fusion. Upon delivery to the cell plate, the SNARE complexes are converted to a fusion-competent form, tentatively through the action of an ATP-consuming $\mathrm{N}$-ethylmaleimide-sensitive factor (NSF) (Karnahl et al. 2017). These factors normally reset the low-energy configuration after fusion has taken place. Disassembled SNARE complexes of different vesicles then form SNARE complexes among each other and trigger vesicle fusion. Specificity for inter-vesicle interactions, as opposed to transitioning back into a low-energy complex still within the same membrane, is facilitated by the SNARE-associated Sec1 protein called KEULE (Park et al. 2012). Together, these findings uncover many of the unique challenges of cell plate biogenesis, but lack the spatial aspect: how does the cell define the plane where vesicles start accumulating and fusing? With our current findings, microtubule overlaps are shown to be excellent platforms to provide this spatial cue for cell plate formation. This then raises the question on how membrane fusion activity can be coupled to these cytoskeletal structures.

\section{Control over membrane accumulation and fusion via tethering factors}

Vesicle tethering complexes have the potential to act as key factors in controlling vesicle fusion machinery at a specific subcellular compartment. Tethering complexes mediate the initial contact between two membranes before fusion. Two recurrent properties of these tethering complexes make them strong candidates for a pivotal role in the local regulation of membrane accumulation and fusion. In the first place, tethering complexes widely engage with the SNARE fusion machinery, and this engagement can exert control over SNARE activity (Hong \& Lev 2014). Also, tethering complexes can similarly interact with SNARE-associated factors, like Sec1proteins (Hashizume et al. 2009; Morgera et al. 2012; Hong \& Lev 2014). Secondly, tethering factors are known effectors of polarity generating mechanisms, which enables their recruitment to a defined area within the cell. This is for example illustrated by cell division in budding yeast, where tethering factors are recruited to the bud neck (Zhang et al. 2008), or by the pathogen Magnaporthe oryzae infecting plant cells, where polarization of tethering factors contributes to the formation of the required invasive structures (Gupta et al. 2015). Localized pools of tethering complexes therefore appear to execute spatial control over membrane fusion activity. 
Two tethering complexes have been implicated in cell plate formation in Arabidopsis: the exocyst and TRAPPII complexes. In chapter 3 we observed that one of the exocyst subunits of moss, Sec6, was positioned by microtubule overlaps during cell division. It also interacted with a Sec1 protein orthologous to Arabidopsis KEULE. These findings support that tethering factors could indeed fulfil a role in tying vesicle fusion activity to a particular subcellular location. It is however too soon to already embed these findings in the molecular framework of SNARE dynamics driving cell plate vesicle fusion. For this, the function of the Sec6-KEULE association needs to be uncovered and the nature and role of the link between Sec6 and overlaps will need to be elucidated. Furthermore, the unknown degree to which mechanisms in moss and Arabidopsis are the same, currently sets a constraint on fully integrating findings from these two model systems.

\section{Establishing the physical link: a role for MAP65?}

The emerging concept that an exocyst component is linked to microtubule overlaps also prompts one to question how such a typically membrane-associated protein could physically associate with these non-membranous structures. By analogy to recruitment strategies in the earlier described animal central spindle, MAP65-family proteins could play a direct role in this. In yeast and animal mitotic microtubule arrays several microtubule regulators are enriched at overlaps via a direct interaction with Ase1/PRC1 bundling proteins (For an overview see Chapter 1; Duellberg et al. 2013). Furthermore PRC1 recruits the centralspindlin complex, a signalling factor essential for cytokinesis in human cells (Gruneberg et al. 2006). Interestingly, in many of these examples the interaction between Ase1/PRC1 and their binding partners can be tuned via phosphorylation/dephosphorylation of the bundling protein (Jiang et al. 1998; Zhu et al. 2006; Fu et al. 2009; Khmelinskii et al. 2009; Hu et al. 2012). A similar regulatory grip on associations that MAP65 engages in, would be a highly useful property in a dynamically reorganizing network such as the phragmoplast. The various phosphorylation sites playing a role in tobacco and Arabidopsis MAP65 functioning (Smertenko et al. 2008; Sasabe \& Machida 2012; Boruc et al. 2017) and some observations on moss MAP65, such as the sharp rise in MAP65labelling occuring at anaphase onset and the altered turnover of MAP65 in close proximity of the forming cell plate (Figure 1), certainly point in the direction of post-translation modification in a cytokinetic context.

Interestingly, a recent study found that in Arabidopsis TRAPPII components Trs120 and Trs130 physically interact with MAP65-family bundling proteins (Steiner et al. 2016). The authors claim that this association does not specifically affect the recruitment of the TRAPPII complex to the phragmoplast midplane, but is rather used by a dividing cell to transmit cell cycle information. They base this on the observation that Trs120 is not mislocalized in a MAP65-3 mutant. However, MAP65-3 function overlaps with that of at least three other Arabidopsis MAP65 paralogs (Sasabe et al. 2011; Li et al. 2017), for one of which (MAP65-1) the authors also show physical association with Trs120 and Trs130 (Steiner et al. 2016). This redundancy thus currently precludes any broader conclusions on the requirement of MAP65s in general for the positioning of the TRAPPII tethering complex.

Despite the several interactions of Ase1/PRC1/MAP65 family proteins documented to date, a common recruitment motif used for association to this family of proteins is currently lacking. In case such a motif could be identified, identification of potential binding partners would be greatly accelerated. 


\section{Membrane partitioning systems outside the plant kingdom}

Partitioning of a cell wall-confined cell into discrete cytoplasms is not a cell biological phenomenon encountered exclusively during cell plate formation in plant cells. The work on analogous systems could provide a framework for the minimal components and their mechanistic interplay during de novo compartmentalization processes. Examples of other known phenomena include the cleavage of a zoosporangium into multiple motile zoospores in Phytophthora sp. (Hyde et al. 1991), embryo cellularization in Drosophila (Mazumdar \& Mazumdar 2002) and the compartmentalization of sporulating yeast cells. The latter is an interesting example, because several molecular aspects of this process have been elucidated. The compartmentalization of sporulating yeast cells occurs through the construction of the so-called forespore or prospore membrane. This membrane envelopes the four post-meiotic nuclei, eventually giving rise to the plasma membrane of the haploid spores (Nakamura et al. 2008). The forespore/prospore membrane compartment is built from vesicles supplied by the secretory pathway that are fused through SNARE action (Shimoda 2004). Interestingly, the construction is initiated at spindle pole bodies, which are yeast equivalents of animal centrosomes (Shimoda 2004). Thus, the repurposing of canonical vesicle trafficking machinery and coordinating fusion activity with microtubule organization is a recurrent theme among unrelated modes of membrane partitioning. This shows that drawing parallels among different systems could help to gain a better understanding of the organizing principles involved in positioning of a de novo membrane compartment for cell separation by the cytoskeleton.

\subsection{Disparity between spindle and phragmoplast design principles}

At first glance the spindle and phragmoplast are architecturally highly similar structures. They are both bipolar microtubule networks of similar size with plus ends pointing towards a central plane. Yet, in chapter 4 we discovered that the processes within these two arrays, in particular microtubule flux, are very different. In this section I will explore how these differences in organizing principles could tie in with the the different functions the spindle and phragmoplast network fulfil.

The metaphase spindle apparatus in moss maintains a strikingly constant length. Controlling this constant length may be a requirement for proper spindle functioning, since apparatus length is an essential determinant of the distance over which chromatids are transported during anaphase (Asbury 2017). Rapid convergence to a steady-state length in the metaphase spindle system is likely facilitated by having a high rate of microtubule turnover (i.e. polymerization and depolymerization, in this case at two distinct sites). In metaphase spindle systems of other organisms it has been shown that organisation is driven by a relatively high rate of polymerization, coupled to microtubule transport and subsequent disassembly (Brugués et al. 2012; Reber et al. 2013; Kapoor 2017). Besides fostering expedient length control, this high turnover likely also facilitates other elemental spindle functions, such as chromosome congression (Wandke et al. 2012; Maiato et al. 2017) and kinetochore-microtubule attachment and error-correction (Lampson \& Grishchuk 2017).

The focus of cytoskeletal processes may be shifted towards controlling overlap length in the phragmoplast, given the essential role of this length in spatial organisation of cytokinesis. Having rapid microtubule dynamics may not be favourable for tight control over overlap length and hence dynamics on overlaps is largely shut down at the onset of cytokinesis. This however comes at the 
expense of control over phragmoplast array length (Chapter 4). The gearing of design principles towards setting up the cytokinetic microtubule overlaps as confined and stable platforms could thus explain the difference with the organizing principles of the metaphase spindle. All in all, adopting a designers perspective on microtubule networks like the phragmoplast and knowing their biological functions can help to identify missing components of network architecture. In the case of the phragmoplast, the still elusive mechanism of controlled microtubule breakdown at the poles and relative alignment of overlaps onto a plane are examples. Vice versa, illuminating what the prime network shaping principles are, can help to rationalize for which demands they are adjusted that particular way.

One interesting implication of thinking of overlaps as stable, autonomous centres for concentrating vesicles and their tethering machinery is that there is no strict requirement for active, motor-driven vesicle transport. Active transport is still part of the textbook explanation of phragmoplast functioning (Evert \& Eichhorn 2013), although experimental evidence supporting this are rather sparse and have not been built upon in the last decade (Lee \& Liu 2000; Otegui et al. 2001). Moreover, results from experiments where synthetic vesicles were micro-injected into dividing cells have shown that these accumulate in the cell-plate region, suggesting that membrane transport mechanisms are highly unspecific or even that accumulation is driven by diffusion and localized capture during plant cytokinesis (Esseling-Ozdoba et al. 2008). An outstanding future challenge is thus to dissect the contributions of active transport, passive transport (i.e. cytosolic flow) and diffusion-based accumulation methods. An excellent starting point to experimentally approach kinesin-based transport would be the extensive information available on kinesin localization during cell division in moss (Miki et al. 2014). Furthermore, a modelling approach could explore if a diffusion-based accumulation mechanism could produce biologically relevant rates of cell-plate membrane delivery, thereby exposing the requirement for active transport.

\subsection{The mechanism of dampening overlap dynamics and its use in tip growth guidance}

Although the evolutionary perspective on the MAP65/kinesin-4 module described in section 6.1 suggests that it has a deeply conserved cytokinetic role, through gene-duplication other, noncytokinetic roles may have additionally evolved. Investigating the functioning of this module in different roles may lead to a better understanding of its working principles. When investigating tip growing cells we found that multiple, dispersed antiparallel overlaps formed within the unipolarized array of these cells (Chapter 5). By developing techniques and markers for tracing polymerisation of microtubules and quantifying tip growth straightness we were able to uncover that these overlaps form a nexus for shaping the microtubule array and ultimately facilitate an adaptable growth axis. That a micron-sized feature in a microtubule network has leverage over the ultimate shape of the organism, opens up opportunities to delve deeper into mechanisms at both ends. Therefore, in this section I will explore how functioning of the crosslinker/kinesin-4 module on microtubules may be regulated at the molecular level, and I will discuss the use of a flexible microtubule array for tip growth guidance.

Overlap length limitation in the interphase microtubule array of tip growing cells is achieved by recruitment of kinesin4-Ia onto MAP65-decorated sites of overlap. The functioning of this module on interphase overlaps shows that, while having an ancient evolutionary history (see 
6.1), it can fulfil versatile functions in entirely different network topologies. At the same time, the findings corroborated the less detailed observations of kinesin- 4 recruitment and action on overlaps of the cytokinetic phragmoplast described in chapter 2. Critically, microscopically capturing the dynamics of antiparallel encounters in the interphase network at high quality allowed us to find striking similarities with the described behaviour of the PRC1 / XKlp1 combination studied in an in vitro system (Bieling et al. 2010). The properties of these antiparallel bundler / kinesin-4 combinations hint that several intricate mechanisms operate at the single molecule level of MAP65 and kinesin- 4 to form such a versatile module on overlaps. Firstly, we observed processive motility of kinesin4-Ia away from sites of MAP65-labelled overlap, but never anywhere else in the microtubule network. This suggests that motility is typically supressed, and that this inhibition is suddenly released as soon as the kinesin-4 molecule is recruited to the overlap. Interestingly, for the human kinesin-4s KIF21A and KIF21B autoinhibitory protein domains in the stalk have been identified (van der Vaart et al. 2013; van Riel et al. 2017). The binding to a microtubule crosslinking protein might dislocate this domain away from the motor domain, thereby abolishing the auto-inhibition and allowing the kinesin-4 to explore microtubules in the overlap (Yount et al. 2015). This brings up a second point where molecular regulation is important: the association between kinesin- 4 and the bundling protein. While physical association between moss MAP65 and kinesin-4 has not yet been directly demonstrated, the deep conservation of the crosslinker / kinesin-4 module makes it highly likely that they directly interact. The putative interaction in moss seems subject to stringent temporal regulation, since both moss kinesin-4s can be prevented from localizing to overlaps at various stages in the cell cycle (Kinesin4-Ic is absent from all overlaps except during cytokinesis and kinesin4-Ia is absent during preprophase and mitosis but present throughout the rest of the cell cycle). In animal cells the dimeric state of the bundling protein (Subramanian et al. 2013) as well as post-translational modification of both the bundling protein and the kinesin-4 (Zhu \& Jiang 2005; Nunes Bastos et al. 2013) are determinants of the interaction at the molecular level. All in all, these multiple layers of control probably make the crosslinker / kinesin-4 module into such a flexible and versatile component for structuring microtubule networks, as observed in plants.

We demonstrate that a lack of kinesin-4-based overlap length restriction has a limiting effect on the amount of possible microtubule orientations (i.e. degrees of freedom) within the interphase network. Without length restriction, microtubules line up along the length axis of cell (i.e. network hyperparallelization). A scenario in which the longitudinal, polarized alignment of microtubules is ending in an apical microtubule focus that dictates the site of tip growth is currently most likely (Doonan et al. 1988; Hiwatashi et al. 2014). To what degree this structure in the apex and microtubules further back in the cell are coupled and how this structure is precisely linked to growth processes, awaits further investigation. Nonetheless, our finding that a more parallelized overall array architecture negatively affects the steerability of tip growth already opens up room to speculate on why a cell would establish a connection between a rather disorderly and ephemeral microtubule structure and a growth process where a certain robustness would be expected. Indeed the polymerization speeds of individual microtubules $(\sim 7 \mu \mathrm{m} / \mathrm{min})$ and the reported median lifetime of the apical microtubule focus (1.6 min; Hiwatashi et al. 2014), strike as rather excessive for a unidirectional growth process occurring at a pace of about 0.35 $\mu \mathrm{m} / \mathrm{min}$. How could a determinate growth process be reconciled with such a rapidly turning over microtubule array with a diversity of filament orientations? If all the cell had to do was to grow straight, then a rigid network with lower rates of turnover would probably suffice. Yet, in a natural setting, changes in the environment (such as flooding and disturbances causing altered 
light / nutrient availability) are encountered. These situations would require swift integration of environmental signals into the growth process. Here, a dynamic ('noisy') array would be highly beneficial, both in the sense of turnover and the amount of possible shapes the network can adopt. A high turnover allows breakdown and rapid replacement of suboptimal configurations and through several iterations convergence towards a more optimal one. Alongside turnover as an ingredient for a tip growing cell to generate new network configurations, one could envisage the range of microtubule orientations in the network as a factor which sets a constraint on the potential diversity of these new network configurations. The hyperalligned microtubules in our kinesin-4 mutant possibly lead to an apical microtubule focus that is too fixed in space to allow redirection of growth processes. Thus, more generally speaking, while diversity in microtubule orientations and microtubule turnover could be considered as noise at the subcellular level, this noise is vital for a cell to come to new decisions on cellular morphogenesis and commit to them. Interestingly, also tissue patterning processes during plant development seem to benefit from a certain degree of internal noise (Meyer \& Roeder 2014), highlighting that fluctuations at lower levels affecting the behaviour of the system at higher levels could be a commonly encountered feature in plant morphogenesis.

\subsection{A perspective on application}

In this thesis I have explored how formation of microtubule overlap regions can shape plant microtubule networks. In an era where an increasing emphasis is put on the flow of information produced by academia to industries and policy making agencies, here I will reflect where the results of this thesis could be translated to such domains. To be brief, chances of these findings producing any offshoot companies, changes in policies, or something in that vein are near-zero. It is however not the case that plant microtubule research in general does not have the potential to bring fruitful insights for biotechnological purposes, as outlined in a recent review (Nick 2012). It certainly also does not mean that there is not a broader context wherein the current work could be embedded, but - just as with any other study investigating the fundamental mechanisms underpinning plant life - this will lie mostly in the development of (scientific) ideas. Before attempting to look ahead in what directions this work could develop, I think it is useful to first look back: what were the major concepts and tools that formed the pillars on which this work could build? (Table 1)

Table 1 - A non-exhaustive list of tools and concepts vital to the work in this thesis

\begin{tabular}{lll} 
Concept / tool & Origin / original research aim & Reference \\
\hline $\begin{array}{l}\text { Cross-bridging of microtubules } \\
\text { within the plant division plane }\end{array}$ & $\begin{array}{l}\text { Fine structure analysis of microtubules during cell } \\
\text { plate formation in the endosperm of African blood lily }\end{array}$ & $\begin{array}{l}\text { (Hepler \& Jackson } \\
\text { 1968) }\end{array}$ \\
\hline $\begin{array}{l}\text { MAP65 as crosslinker of phragmo- } \\
\text { plast microtubules }\end{array}$ & $\begin{array}{l}\text { Screen of microtubule associated proteins in tobacco } \\
\text { and carrot cells; Examination of a 'PLEIADE' Arabi- } \\
\text { dopsis mutant. }\end{array}$ & $\begin{array}{l}\text { (Chang-Jie \& } \\
\text { Sonobe 1993; Chan } \\
\text { et al. 1999; Müller et } \\
\text { al. 2004) }\end{array}$ \\
$\begin{array}{lll}\text { A crosslinker / kinesin-4 combina- } \\
\text { tion for length control }\end{array}$ & $\begin{array}{l}\text { Understanding overlap length control in the animal } \\
\text { central spindle }\end{array}$ & $\begin{array}{l}\text { (Bieling et al. 2010; } \\
\text { Hu et al. 2011) }\end{array}$
\end{tabular}

Possible cytokinetic functions of moss kinesin-4s 
Table 1 - A non-exhaustive list of tools and concepts vital to the work in this thesis

\begin{tabular}{lll} 
Concept / tool & Origin / original research aim & Reference \\
\hline Photo-activatable GFP & $\begin{array}{l}\text { Exploitation of a mutated residue in wild-type GFP } \\
\text { that deleted one of the two excitation bands present } \\
\text { in GFP }\end{array}$ & $\begin{array}{l}\text { (Ehrig et al. 1995; } \\
\text { Patterson \& Lippin- } \\
\text { cott-Schwartz 2002) }\end{array}$
\end{tabular}

Physcomitrella patens $\quad$ Understanding genetic basis of plant autotrophy $\quad$ (Engel 1968)

\begin{tabular}{lll}
$\begin{array}{l}\text { Homologous recombination in } P . \\
\text { patens }\end{array}$ & $\begin{array}{l}\text { Exploring why strong genetic linkage exists between } \\
\text { two successively introduced constructs }\end{array}$ & $\begin{array}{l}\text { (Kammerer \& Cove } \\
1996)\end{array}$ \\
\hline Spinning Disc / Nipkow Disc & $\begin{array}{l}\text { Early investigations into transmission of images by } \\
\text { electrical signals / 'mechanical television' }\end{array}$ & $\begin{array}{l}\text { (Tanaami et al. } \\
\text { 2002) }\end{array}$ \\
ImageJ image analysis software & $\begin{array}{l}\text { (Ongoing) development of an early image analysis } \\
\text { program used mainly by medical sciences }\end{array}$ & $\begin{array}{l}\text { (Schneider et al. } \\
\text { 2012) }\end{array}$ \\
\hline
\end{tabular}

While this inventory is by no means complete or illustrative of all scientific endeavours, from it emerges a trend that only in select cases the application in the current work of previously developed concepts is matching the use anticipated or intended by the original researcher(s). In other cases, such as the identification of MAP65 or moss kinesin-4, it was 'just' the outcome of a large scale screen. Yet, despite the evidently erratic flux of ideas and tools, the a priori assessment of potential societal impact has become a significant part of raising funds for scientific programmes. Being investments made with public money, effective oversight of the value created by scientific programmes is off course in place. However, metrics currently used for the assessment of impact, revolve around whether any direct solutions to problems facing humanity are created by a scientific programme. I see two problems when emphasis is put on this type of metric. It will inflate public expectations ("Why are there still pests, food shortages, etc.?”), which will damage the relation between scientific community and public in the long run. In addition to this, it narrows the mind and focus of a researcher. Certain input ideas may be ignored, because they are judged against the prevailing valorisation metric ("What possible use could studying the heritable immune system of prokaryotes ever have?”).

Another notion illustrated by table 1 is that application may not be bound to a discipline or may come at a much later time. For example, the Nipkow disc found its main application in early television systems and was rapidly superseded by superior mechanisms, only to resurface decades later as an exceptionally useful principle to illuminate living specimens on a microscope. This 'serendipity' makes it hard to look ahead and write down opportunities that could be logically expected to flow from the work described in this thesis. One could certainly speculate how, perhaps, the mutants with a defective cell-cell interface generated in chapter 2 may prove useful for plasmodesmata or cell-cell communication research. How maybe the uncovered role of overlaps in a diffusely organized, unipolarized network can lend insights into the self-organization of other polymer networks in long, slender cells. However, while being potential ideas that can bring value to other fields, they are still empty promises, making the maximization of the chance that they reach these other fields a meaningful commitment. Fostering dissemination of ideas to other places and other minds should therefore be something we aspire to. 


\section{References}

Ahn, G. et al., 2017. SH3 domain-containing protein 2 plays a crucial role at the step of membrane tubulation during cell plate formation. The Plant Cell, 29(6), pp.1388-1405.

Asbury, C., 2017. Anaphase A: Disassembling microtubules move chromosomes toward spindle poles. Biology (Basel), 6(1), p.15.

Austin, J.R., Seguí-Simarro, J.M. \& Staehelin, L.A., 2005. Quantitative analysis of changes in spatial distribution and plus-end geometry of microtubules involved in plant-cell cytokinesis. Journal of Cell Science, 118(17), pp.3895-903.

Bieling, P., Telley, I. \& Surrey, T., 2010. A minimal midzone protein module controls formation and length of antiparallel microtubule overlaps. Cell, 142(3), pp.420-432.

Boruc, J. et al., 2017. Phosphorylation of MAP65-1 by Arabidopsis Aurora kinases is required for efficient cell cycle progression. Plant Physiology, 173(1), pp.582-599.

Boruc, J. \& Van Damme, D., 2015. Endomembrane trafficking overarching cell plate formation. Current Opinion in Plant Biology, 28, pp.92-98.

Brugués, J. et al., 2012. Nucleation and transport organize microtubules in metaphase spindles. Cell, 149(3), pp.554-564.

Buschmann, H. \& Zachgo, S., 2016. The evolution of cell division: From streptophyte algae to land plants. Trends in Plant Science, 21(10), pp.872-883.

Butterfield, N.J., 2015. Early evolution of the Eukaryota. Palaeontology, 58(1), pp.5-17.

Chan, J. et al., 1999. The $65-\mathrm{kD}$ a carrot microtubule-associated protein forms regularly arranged filamentous cross-bridges between microtubules. Proceedings of the National Academy of Sciences of the United States of America, 96(26), pp.14931-14936.

Chang-Jie, J. \& Sonobe, S., 1993. Identification and preliminary characterization of a $65 \mathrm{kDa}$ higher-plant microtubule-associated protein. Journal of Cell Science, 105, pp.891-901.

Doonan, J., Cove, D.J. \& Lloyd, C., 1988. Microtubules and microfilaments in tip growth: evidence that microtubules impose polarity on protonemal growth in Physcomitrella patens. Journal of Cell Science, 89, pp.533-540.

Duellberg, C. et al., 2013. End-binding proteins and Ase1/PRC1 define local functionality of structurally distinct parts of the microtubule cytoskeleton. Trends in Cell Biology, 23(2), pp.54-63.

Ehrig, T., O'Kane, D.J. \& Prendergast, F.G., 1995. Green-fluorescent protein mutants with altered fluorescence excitation spectra. FEBS letters, 367(2), pp.163-166.

Eme, L. et al., 2014. On the age of eukaryotes: evaluating evidence from fossils and molecular clocks. Cold Spring Harbor Perspectives in Biology, 6(8), p.a016139.

Engel, P.P., 1968. The induction of biochemical and morphological mutants in the moss Physcomitrella patens. American Journal of Botany, 55(4), pp.438-446.

Esseling-Ozdoba, A. et al., 2008. Synthetic lipid (DOPG) vesicles accumulate in the cell plate region but do not fuse. Plant Physiology, 147(4), pp.1699-1709.

Euteneuer, U. \& Mcintosh, J.R., 1980. Polarity of midbody and phragmoplast microtubules. Journal of Cell Biology, 87, pp.509-515.

Evert, R.F. \& Eichhorn, S.E., 2013. Raven biology of plants, 8th edn, W.H. Freeman/Palgrave Macmillan.

Flemming, W., 1891. Neue Beiträge zur Kenntniss der Zelle. II Theil. Archiv für Mikroskopische Anatomie, 37(1), pp.685-751.

Fowke, L.C. \& Pickett-Heaps, J.D., 1969. Cell division in Spirogyra. II. Cytokinesis. Journal of Phycology, 5(4), pp.273-281.

Fu, C. et al., 2009. Phospho-regulated interaction between kinesin-6 Klp9p and microtubule bundler Ase1p promotes spindle elongation. Developmental Cell, 17(2), pp.257-267.

Glotzer, M., 2009. The 3Ms of central spindle assembly: microtubules, motors and MAPs. Nature Reviews: Molecular Cell Biology, 10(1), pp.9-20.

Gruneberg, U. et al., 2006. KIF14 and citron kinase act together to promote efficient cytokinesis. Journal of Cell Biology, 172(3), pp.363-372.

Gupta, Y.K. et al., 2015. Septin-dependent assembly of the exocyst is essential for plant infection by Magnaporthe oryzae. The Plant Cell, 27(11), pp.3277-3289.

Hashizume, K. et al., 2009. Yeast Sec1p functions before and after vesicle docking. Molecular Biology of the Cell, 20(22), pp.4673-4685. 
Hepler, P.K. \& Jackson, W.T., 1968. Microtubules and early stages of cell-plate formation in the endosperm of Haemanthus katherinae Baker. Journal of Cell Biology, 38(2), pp.437-446.

Hiwatashi, Y., Sato, Y. \& Doonan, J.H., 2014. Kinesins have a dual function in organizing microtubules during both tip growth and cytokinesis in Physcomitrella patens. The Plant Cell, 26(3), pp.1256-1266.

Ho, C.-M.K. et al., 2012. Arabidopsis microtubule-associated protein MAP65-3 cross-links antiparallel microtubules toward their plus ends in the phragmoplast via its distinct $\mathrm{C}$-terminal microtubule binding domain. The Plant Cell, 24(5), pp.2071-2085.

Ho, C.-M.K. et al., 2011. Interaction of antiparallel microtubules in the phragmoplast is mediated by the microtubule-associated protein MAP65-3 in Arabidopsis. The Plant Cell, 23(8), pp.2909-2923.

Hong, W. \& Lev, S., 2014. Tethering the assembly of SNARE complexes. Trends in Cell Biology, 24(1), pp.35-43.

Hong, Z. et al., 2003. Phragmoplastin dynamics: multiple forms, microtubule association and their roles in cell plate formation in plants. Plant Molecular Biology, 53(3), pp.297-312.

Hu, C.-K. et al., 2011. KIF4 regulates midzone length during cytokinesis. Current Biology, 21(10), pp.815824.

Hu, C.-K. et al., 2012. Plk1 negatively regulates PRC1 to prevent premature midzone formation before cytokinesis. Molecular Biology of the Cell, 23(14), pp.2702-2711.

Hyde, G.J. et al., 1991. Freeze substitution reveals a new model for sporangial cleavage in Phytophthora, a result with implications for cytokinesis in other eukaryotes. Journal of Cell Science, 100(4), pp.735-746.

Jiang, W. et al., 1998. PRC1: a human mitotic spindle-associated CDK substrate protein required for cytokinesis. Molecular Cell, 2(6), pp.877-885.

Jürgens, G. et al., 2015. Plant cytokinesis: a tale of membrane traffic and fusion. Biochemical Society Transactions, 43(1), pp.73-78.

Kammerer, W. \& Cove, D.J., 1996. Genetic analysis of the effects of re-transformation of transgenic lines of the moss Physcomitrella patens. Molecular \& General Genetics, 250(3), pp.380-382.

Kapoor, T., 2017. Metaphase spindle assembly. Biology (Basel), 6(1), p.8.

Karnahl, M. et al., 2017. ER assembly of SNARE complexes mediating formation of partitioning membrane in Arabidopsis cytokinesis. eLife, 6, e25327.

Katsaros, C.I. et al., 2011. Comparative immunofluorescence and ultrastructural analysis of microtubule organization in Uronema sp., Klebsormidium flaccidum, K. subtilissimum, Stichococcus bacillaris and S. chloranthus (Chlorophyta). Protist, 162(2), pp.315-331.

de Keijzer, J. et al., 2017. Shortening of microtubule overlap regions defines membrane delivery sites during plant cytokinesis. Current Biology, 27(4), pp.514-520.

Khmelinskii, A. et al., 2009. Phosphorylation-dependent protein interactions at the spindle midzone mediate cell cycle regulation of spindle elongation. Developmental Cell, 17(2), pp.244-256.

Knoll, A.H. \& Nowak, M.A., 2017. The timetable of evolution. Science Advances, 3(5), pp.1-13.

Kong, Z. et al., 2015. Kinesin-4 functions in vesicular transport on cortical microtubules and regulates cell wall mechanics during cell elongation in plants. Molecular Plant, 8(7), pp.1011-1023.

Kurasawa, Y. et al., 2004. Essential roles of KIF4 and its binding partner PRC1 in organized central spindle midzone formation. The EMBO Journal, 23(16), pp.3237-3248.

Lampson, M. \& Grishchuk, E., 2017. Mechanisms to avoid and correct erroneous kinetochore-microtubule attachments. Biology (Basel), 6(1), p.1.

Lauber, M.H. et al., 1997. The Arabidopsis KNOLLE protein is a cytokinesis-specific syntaxin. Journal of Cell Biology, 139(6), pp.1485-1493.

Lee, K.Y., Davies, T. \& Mishima, M., 2012. Cytokinesis microtubule organisers at a glance. Journal of Cell Science, 125(15), pp.3495-3500.

Lee, Y.R. \& Liu, B., 2000. Identification of a phragmoplast-associated kinesin-related protein in higher plants. Current Biology, 10(13), pp.797-800.

Lekomtsev, S. et al., 2012. Centralspindlin links the mitotic spindle to the plasma membrane during cytokinesis. Nature, 492(7428), pp.276-279.

Li, H. et al., 2017. Arabidopsis MAP65-4 plays a role in phragmoplast microtubule organization and marks the cortical cell division site. New Phytologist, 215(1), pp.187-201.

Maiato, H. et al., 2017. Mechanisms of chromosome congression during mitosis. Biology (Basel), 6(1), p.13.

Mazumdar, A. \& Mazumdar, M., 2002. How one becomes many: blastoderm cellularization in Drosophila melanogaster. BioEssays, 24(11), pp.1012-1022. 
Mcmichael, C.M. \& Bednarek, S.Y., 2013. Cytoskeletal and membrane dynamics during higher plant cytokinesis. New Phytologist, 197(2013), pp.1039-1057.

Meyer, H.M. \& Roeder, A.H.K., 2014. Stochasticity in plant cellular growth and patterning. Frontiers in Plant Science, 5 , p.420.

Miki, T. et al., 2014. Endogenous localizome identifies 43 mitotic kinesins in a plant cell. Proceedings of the National Academy of Sciences of the United States of America, 111(11), pp.1053-1061.

Mishima, M., Kaitna, S. \& Glotzer, M., 2002. Central spindle assembly and cytokinesis require a kinesinlike protein/RhoGAP complex with microtubule bundling activity. Developmental Cell, 2(1), pp.41-54.

Morgera, F. et al., 2012. Regulation of exocytosis by the exocyst subunit Sec6 and the SM protein Sec1. Molecular Biology of the Cell, 23(2), pp.337-346.

Müller, S. et al., 2004. The plant microtubule-associated protein AtMAP65-3/PLE is essential for cytokinetic phragmoplast function. Current Biology, 14(5), pp.412-417.

Nakamura, T. et al., 2008. Live observation of forespore membrane formation in fission yeast. Molecular Biology of the Cell, 19(8), pp.3544-3553.

Nick, P., 2012. Microtubules and the tax payer. Protoplasma, 249(2), pp.81-94.

Nunes Bastos, R. et al., 2013. Aurora B suppresses microtubule dynamics and limits central spindle size by locally activating KIF4A. Journal of Cell Biology, 202(4), pp.605-621.

Otegui, M.S. et al., 2001. Three-dimensional analysis of syncytial-type cell plates during endosperm cellularization visualized by high resolution electron tomography. The Plant Cell, 13(9), pp.2033-2051.

Otegui, M.S., Verbrugghe, K.J. \& Skop, A.R., 2005. Midbodies and phragmoplasts: analogous structures involved in cytokinesis. Trends in Cell Biology, 15(8), pp.404-413.

Park, M. et al., 2012. Sec1/Munc18 protein stabilizes fusion-competent syntaxin for membrane fusion in Arabidopsis cytokinesis. Developmental Cell, 22(5), pp.989-1000.

Patterson, G.H. \& Lippincott-Schwartz, J., 2002. A photoactivatable GFP for selective photolabeling of proteins and cells. Science, 297(5588), pp.1873-1877.

Pickett-heaps, J.D. et al., 1999. The cytoplast concept in dividing plant cells: cytoplasmic domains and the evolution of spatially organized cell. American Journal of Botany, 86(2), pp.153-172.

Pickett-Heaps, J.D., 1975. Green algae: Structure, reproduction and evolution in selected genera, Sinauer Associates.

Reber, S.B. et al., 2013. XMAP215 activity sets spindle length by controlling the total mass of spindle microtubules. Nature Cell Biology, 15(9), pp.1116-1122.

Richter, S. et al., 2014. Delivery of endocytosed proteins to the cell-division plane requires change of pathway from recycling to secretion. eL ife, 3, e02131.

van Riel, W.E. et al., 2017. Kinesin-4 KIF21B is a potent microtubule pausing factor. eLife, 6, e24746.

Sasabe, M. et al., 2011. Arabidopsis thaliana MAP65-1 and MAP65-2 function redundantly with MAP65-3/ PLEIADE in cytokinesis downstream of MPK4. Plant Signaling \& Behavior, 6(5), pp.743-747.

Sasabe, M. \& Machida, Y., 2012. Regulation of organization and function of microtubules by the mitogenactivated protein kinase cascade during plant cytokinesis. Cytoskeleton, 69(11), pp.913-918.

Schneider, C.A., Rasband, W.S. \& Eliceiri, K.W., 2012. NIH Image to ImageJ: 25 years of image analysis. Nature Methods, 9(7), pp.671-675.

Seguí-Simarro, J.M. et al., 2004. Electron tomographic analysis of somatic cell plate formation in meristematic cells of Arabidopsis preserved by high-pressure freezing. The Plant Cell, 16(4), pp.836-856.

Shimoda, C., 2004. Forespore membrane assembly in yeast: coordinating SPBs and membrane trafficking. Journal of Cell Science, 117(3), pp.389-396.

Smertenko, A.P. et al., 2008. The C-terminal variable region specifies the dynamic properties of Arabidopsis microtubule-associated protein MAP65 isotypes. The Plant Cell, 20(12), pp.3346-3358.

Steiner, A. et al., 2016. Cell cycle-regulated PLEIADE/AtMAP65-3 links membrane and microtubule dynamics during plant cytokinesis. The Plant Journal, 88(4), pp.531-541.

Subramanian, R. et al., 2013. Marking and measuring single microtubules by PRC1 and kinesin-4. Cell, 154(2), pp.377-390.

Tanaami, T. et al., 2002. High-speed 1-frame/ms scanning confocal microscope with a microlens and Nipkow disks. Applied Optics, 41(22), pp.4704-4708.

Tomei, E.J. \& Wolniak, S.M., 2016. Transcriptome analysis reveals a diverse family of kinesins essential for spermatogenesis in the fern Marsilea. Cytoskeleton, 73(3), pp.145-159.

van der Vaart, B. et al., 2013. CFEOM1-associated kinesin KIF21A is a cortical microtubule growth inhibitor. Developmental Cell, 27(2), pp.145-160. 
Vernos, I. et al., 1995. Xklp1, a chromosomal Xenopus kinesin-like protein essential for spindle organization and chromosome positioning. Cell, 81(1), pp.117-127.

Waizenegger, I. et al., 2000. The Arabidopsis KNOLLE and KEULE genes interact to promote vesicle fusion during cytokinesis. Current Biology, 10(21), pp.1371-1374.

Wandke, C. et al., 2012. Human chromokinesins promote chromosome congression and spindle microtubule dynamics during mitosis. Journal of Cell Biology, 198(5), pp.847-863.

Williams, B.C. et al., 1995. The Drosophila kinesin-like protein KLP3A is a midbody component required for central spindle assembly and initiation of cytokinesis. Journal of Cell Biology, 129(3), pp.709-723.

Williams, T.A., 2014. Evolution: Rooting the eukaryotic tree of life. Current Biology, 24(4), pp.151-152.

Yount, A.L., Zong, H. \& Walczak, C.E., 2015. Regulatory mechanisms that control mitotic kinesins. Experimental Cell Research, 334(1), pp.70-77.

Zhang, X. et al., 2008. Membrane association and functional regulation of Sec3 by phospholipids and Cdc42. Journal of Cell Biology, 180(1), pp.145-158.

Zhu, C. et al., 2006. Spatiotemporal control of spindle midzone formation by PRC1 in human cells. Proceedings of the National Academy of Sciences of the United States of America, 103(16), pp.6196-6201.

Zhu, C. \& Jiang, W., 2005. Cell cycle-dependent translocation of PRC1 on the spindle by Kif4 is essential for midzone formation and cytokinesis. Proceedings of the National Academy of Sciences of the United States of America, 102(2), pp.343-348. 


\section{Summary}

Microtubules are long filaments made up from protein building blocks and ubiquitously employed by eukaryotic cells for a wide range of often essential cellular processes. To perform these functions, microtubules are virtually always organized into higher order networks. Microtubule networks in cells of land plants are fundamental for guiding growth processes and for bringing about their unique mode of cell division. The latter is facilitated by the so-called phragmoplast network, consisting of two opposing sets of microtubules that foster in their centre the formation and radial outgrowth of a disc-shaped membrane compartment (termed the cell plate) that ultimately divides the two daughter cells. The mechanisms driving the spatial organisation of such networks are of outstanding interest because plant cells do not rely on major microtubule organizers as in most other organisms. Instead, plant cells use a wide range of dispersed interactions among individual microtubules to shape functional microtubule networks. Chapter 1 introduces encounters between microtubules of opposite polarity and consequent bundling as potentially powerful handles to organize microtubules into networks. These encounters generate an area of antiparallel microtubule overlap and such overlaps are a striking feature of the phragmoplast microtubule network.

For long it is recognized that the short overlaps formed among the two opposing sets of phragmoplast microtubules and the membranous structures of the cell plate fall within the same plane. In chapter 2 we hypothesize that the limited length of these overlaps is required for the confined accumulation of cell plate membranes. To investigate this, we start out by co-visualizing overlaps and cell-plate membrane material in living cells of the moss Physcomitrella patens, an emerging model plant system with a convenient genetic toolset and tissues readily observable through microscopy. We reaffirm an early association between overlaps and membranes and further explored this association by experimentally altering overlap length. Incited by length control mechanisms of overlaps in animal cells, we identify two kinesin-4 motor proteins that jointly limit the length of phragmoplast microtubule overlaps in moss. Using cells lacking these kinesin-4s we then show that over-elongation of microtubule overlaps leads to a broadening of initial cell plate membrane depositions and a delayed progression of radial cell plate outgrowth. The cross walls ultimately formed by the wider membrane depositions were found to be thick and irregularly shaped. We thus demonstrate that kinesin-4-dependent overlap shortening in the phragmoplast defines the site of cell plate synthesis for the proper scaffolding of a new cell wall segment separating two daughter cells.

In chapter 3 we further investigate molecular mechanisms that could explain how linkage between a microtubule overlap and membrane assembly activity is realized. We focus on the exocyst tethering complex, one of the membrane tethering complexes involved in cell plate formation in flowering plants. We survey the localization of several moss exocyst subunits during cell division and find that one (Sec6) localizes to microtubule overlaps already before the onset of cell plate biogenesis. Experiments in which overlap length is altered and overlap formation is suppressed reveal that these structures play an important role in positioning Sec6 during cell division. The ability of moss Sec6 to interact with an evolutionary conserved factor in cell plate membrane fusion called KEULE is demonstrated, signifying a potential functional link between membrane tethering and fusion activities during cell plate formation. The precise role of Sec6 positioning by overlaps is as yet unclear, but in the light of the importance of overlaps for spatial control of cytokinesis will prove to be an intriguing direction for future research efforts. 
In chapter 4 we gain further mechanistic insight in kinesin- 4 mediated overlap length control and governance of division apparatus length as a whole. We focus on microtubule growth in overlaps regulated by kinesin-4, the poleward transport of microtubule polymers (termed flux), and the interplay between these processes. First, a method based on localized photoactivation is established for the quantitative assessment of microtubule flux. We demonstrate that initially flux in the metaphase spindle occurs synchronized and at high rates, to be replaced by a heterogeneous and on average much slower microtubule flux in the phragmoplast. Since polymerisation of microtubules could provide direct fuel for flux, we postulate that the rate of microtubule growth at sites of overlap could determine flux rates. To test this, we experimentally enhance polymerisation rates through knock-out of kinesin-4 proteins. This approach is validated by experiments demonstrating that they can supress microtubule outgrowth at overlaps in an in vivo setting. Upon kinesin-4 removal, flux rates are enhanced signifying coupling to rates of polymerization. We also find that lack of kinesin-4s leads elongation of the entire division apparatus and that this length change is proportional to the temporal activity patterns of the two kinesin-4s. Based on these findings we propose a mechanism for length regulation through a balance of microtubule growth in the overlap zone, retrograde microtubule translocation and putatively microtubule breakdown at the poles. Microtubule turnover in this system is high in the metaphase spindle $(\sim 1.5 \mu \mathrm{m} / \mathrm{min})$, which, partly through kinesin- 4 action, is succeeded by a more slowly turning over system in the form of the phragmoplast.

While in general the involvement of antiparallel microtubule overlaps in spatial organization of bipolar microtubule configurations is evident, how they could help shape other geometries is largely unknown. Chapter $\mathbf{5}$ starts out with the observation that within the unipolarized microtubule array of tip growing moss cells during interphase, there is occasional formation of overlaps at dispersed sites in the network. Tip growth is a mode of growth allowing rapid colonization of the environment and is achieved through highly polarized secretion, whereby the microtubule network reportedly steers the grows axis. We identify one kinesin-4 motor (Kin4Ia) recruited to the observed overlaps within this network and use knock-out of Kin4-Ia to assess its role in tip growth. This reveals that absence of Kin4-Ia leads to a less adaptable axis of tip growth, prompting further investigation of Kin4-Ia behaviour at interphase overlaps. We find that this kinesin- 4 is recruited with a slight delay to overlaps after their formation and inhibits plus end polymerization of overlap microtubules, thereby limiting overlap length. We then uncover that this activity helps to keep the network polarized towards the tip and prevent the overall organization from becoming hyperaligned with the cell axis. We propose that the latter observation might explain the decrease in growth axis adaptability.

Overall, this thesis demonstrates that in plant microtubule networks of varying architecture, the formation of antiparallel overlaps provides a defined network feature for the recruitment of other microtubule-based process. Together, overlaps and activities coordinated from there, are potent organizers of functional plant microtubule arrays. The potential wider implications of these findings, their relationship to membrane-bound cytokinetic processes, and their evolutionary context are briefly discussed in Chapter $\mathbf{6}$. 


\title{
Acknowledgements
}

\author{
"6very adventure requires a first step. Trite, but true, even here." -Cheshire Cat
}

Marcel, ik was in het begin enigszins onthutst dat je een aio-project binnen cel biologie wilde creëren en mij daarbij wilde betrekken. Het bleek een eerste stap op een pad dat veel voldoening heeft gegeven. Vanaf het eerste moment heb ik veel vrijheid en vertrouwen genoten, waarvoor dank. Daarnaast heb je vele mogelijkheden voor me geschapen om andere mensen in het veld te leren kennen en om mijn werk te kunnen presenteren. Met name de tijden in Amerika zijn unieke en vormende periodes geweest. De schrijf-momenten samen ontaarden vaak in verhitte discussies waar ik veel van heb genoten, en wat mijn schrijven (hopelijk) wat scherper heeft gemaakt.

Tijs, ik heb veel waardering dat je altijd wat tijd wist te maken voor gesprekken en adviezen over onderzoeksstrategieën, kloneerproblemen, mogelijke carrière-stappen en van alles en nog wat. Jouw tomeloos optimisme en flinke dosis pragmatisme zijn essentieel geweest om lekker door te kunnen werken.

The cradle of this thesis is interestingly enough not in Wageningen, but stands firmly on Japanese soil. Goshima-san, thank you for hosting me and learning me a wealth of new things. Thanks for the overwhelming hospitality (From operating Shinkansen ticket machines, double checking the length of the bed in my apartment to organizing Bōnenkai). For picking up the finer arts of moss cultivation I'm greatly indebted to Nakaoka-san, Tomo and Kosetsu-san. Thanks to Smilie-san for arranging much of the paperwork. Further, I'd like to thank all Goshima lab members for a great time in the lab, and for all the sportive/sightseeing/culinary things we did together. You've made the start of my $\mathrm{PhD}$ a highly enjoyable experience; the Dutch saying 'een goed begin is het halve werk', definitely applies here.

I owe a big thank you to all members of the Cell Biology department, past and present, for your help and for your companionship throughout my $\mathrm{PhD}$ period.

Henk, na terugkeer uit Japan ben jij heel belangrijk geweest om de waslijst aan instrumenten specifiek voor Physco om te zetten in een goed lopend mos-lab. Maar ook daarna wisten we samen met succes veel voor elkaar krijgen, zoals het bouwen van mos-koelkasten, het vinden van knock-out mutanten en met de electronenmicroscoop kijken naar tussenwandjes. Ik waardeer je hulpvaardigheid en collegialiteit van de afgelopen jaren enorm. Norbert, dank voor je assistentie bij de microscopen en om de 'mos-vergunning' rond te krijgen. Bela, ik heb veel bewondering voor je aanstekelijk vermogen om verder te vragen, lunch te initiëren en situaties van Monty Python quotes te voorzien. Het was leuk om samen een review te maken en ik hoop dat uit het tipgroei project ook nog wat moois volgt. Marie-José, dank je wel voor de ondersteuning en alles wat je deed om de gezamenlijke cadeaus, kerst, en labuitjes mogelijk te maken. André, met het 
afmaken van de MSc thesis die ik bij je deed heb je veel energie gestoken om het kastanje project een mooie plek te geven (in veel opzichten). Buiten het feit dat ik het hierbij erg naar mijn zin heb gehad, heeft deze periode samen met de vakken die je gaf en de Physco-lijnen die je al vroeg naar Wageningen hebt gehaald de omstandigheden en voorwaarden geschapen om aan precies deze $\mathrm{PhD}$ te beginnen. Kris, onze wegen zijn een poos geleden vervlochten geraakt in Wageningen en daar ben ik nog steeds heel dankbaar voor. Juliane en Olya, bedankt voor de gezelligheid aan het begin van mijn $\mathrm{PhD}$ periode en Hannie en Alejandra voor de gezelligheid later; jullie zorg(d)en voor een welkom gevoel in de groep! Aniek, dankjewel voor het samensmeden van dan wel een we-day team, dan wel een pub quiz team uit onze toch niet al te grote club mensen. Je creatieve en droge humor (soms geïnspireerd door kiwi-binnenkanten) is onvervangbaar! Elysa, je bracht Physco samen met een oude bekende, Phytophthora, een succesvolle combinatie waarvan ik blij ben dat ik het één en ander heb kunnen bijdragen; veel succes met dit duo verder. Jelmer, dankjewel dat je me hebt geïntroduceerd in de wondere wereld van MATLAB; het is een onmisbaar instrument van mijn onderzoek geworden.

Han, when we found an utterly peculiar localization pattern during cell division for one of the exocyst subunits you were studying, a whole new project was founded. Via winding roads and much of your hard work this has now come to a nice manuscript. All along you were a great neighbour that rekindled my appreciation for the 'tosti'. I hope your new neighbour will keep a cleaner desk than I did!

I've had great pleasure in working together with MSc students during their thesis work. Jorrit, Rudolf, Gonda, Xiaoxue, Joanne, Chaniël and Ruben, you've contributed a lot, but above all, brought a lot of joy to the workplace. Also Rik Brugman, Lisa, Harsh, Sjoerd, Els, Maikel, and all other students and fridays AMOLF-visitors that joined the group, thanks for bringing in your liveliness.

Dolf en Maritza, dank dat jullie langs kwamen met het idee om een bijzondere klasse van planten eiwitten eens nader te gaan bekijken in Physco. Een interessant project dat voor mij de kracht van het breder kijken in 'the green kingdom' definitief bevestigde.

With coffee-breaks it was nice to have $\mathrm{PhD}$-students and other people of the MolBi and PDB neighbouring groups around. Thanks also for the shared labtrips and Christmas dinners. Sabine, Rik, Arjan, Ting Ting, Adam and Bandan, thanks for the shared beers and highly enjoyable company on Friday nights!

Om verder de zin op andere zaken te zetten zijn in het begin Anco, Marcel, Jan en Peter belangrijk geweest tussen het kleinfruit in de Betuwe op zaterdagen. Later werden de zaterdagen gevuld met klussen in de gemeenschappelijke tuin met 'Minny en haar mannen'. Beide onmisbare afleidingen en geweldige andere manieren om met planten bezig te zijn. Ik heb ook met veel plezier gevolleybald bij de recreanten van Invicta. Met een goede sfeer een balletje slaan op maandagavond en daarna nog even kijken of we de bar open konden krijgen. Iedereen die dat mogelijk maakte, in het bijzonder Piet en Trudi, dank jullie wel. 
De buren, en meer specifiek mijn clustergenoten, waren onmisbaar voor een steuntje in de rug en het scheppen van ongetwijfeld de fijnste plek om te wonen in Wageningen. Fen, Giel, Lillemaj, Marg, Anita, Jeltje (en later gelukkig ook Shiva!) Bonnie, Jorden, Rinske en Emil, dank. En dan niet te vergeten mijn èchte buurvrouw, Marijn; je combinatie van een rijke fantasie, mafheid, ernst en inlevingsvermogen makkten dat ik meer dan eens blij thuis kwam. Jorin, ik heb veel opgestoken van de $\mathrm{PhD}$-ervaringen die jij deelde en genoten van de discussies en spelletjes. Veel dank verder aan alle vrienden voor de belangstelling en aanmoediging.

Het continue werken in een niche die ver af staat van het dagelijkse (ver 'down the rabbit hole' om ook in Alice's adventures in Wonderland termen te eindigen) zorgde ervoor dat ik me er meer dan eens door moest laten opslokken: Het 'afronden' van projecten en lange pozen in het buitenland bivakkeren. Dank aan mijn ouders, mijn zusjes en Kiki voor het geduld wat dit ongetwijfeld heeft gevergd. Aan de andere kant leren die momenten wel dat jullie heel belangrijk voor me zijn! 


\section{List of publications}

de Keijzer J, Kieft H, Ketelaar T, Goshima G, Janson ME. Shortening of microtubule overlap regions defines membrane delivery sites during plant cytokinesis. (2017) Current Biology 27, p. $514-520$

Overdijk EJ, de Keijzer J, de Groot D, Schoina C, Bouwmeester K, Ketelaar T, Govers F. Interaction between the moss Physcomitrella patens and Phytophthora: a novel pathosystem for live-cell imaging of subcellular defence. (2016) Journal of Microscopy 263, p. 171-180.

de Keijzer J, Mulder BM, Janson ME. Microtubule networks for plant cell division. (2014) Systems and Synthetic Biology 8, p.187-194.

Kosetsu K, de Keijzer J, Janson ME, Goshima G. MICROTUBULE-ASSOCIATED PROTEIN65 is essential for maintenance of phragmoplast bipolarity and formation of the cell plate in Physcomitrella patens. (2013) Plant Cell 25, p. 4479-4492.

Hua C, Meijer HJ, de Keijzer J, Zhao W, Wang Y, Govers F. GK4, a G-protein-coupled receptor with a phosphatidylinositol phosphate kinase domain in Phytophthora infestans, is involved in sporangia development and virulence. (2013) Molecular Microbiology 88, p. 352-370.

de Keijzer J, van den Broek LA, Ketelaar T, van Lammeren AA. Histological examination of horse chestnut infection by Pseudomonas syringae pv. aesculi and non-destructive heat treatment to stop disease progression. (2012) PLoS One 7, e39604.

de Keijzer J, van Spoordonk R, van der Meer-Verweij JE, Mulder BM, Janson ME and Ketelaar T. Kinesin-4 optimizes microtubule orientations for responsive tip growth guidance in moss protonemata. Manuscript in preparation 


\section{Curriculum vitae}

Jeroen de Keijzer was born (on September 20, 1987) and raised in the Betuwe area of the Netherlands. He attended secondary education at the 'Rijksscholengemeenschap Lingecollege' in Tiel, which he finalized in 2005. He then started a BSc study in biology at Wageningen University, the Netherlands. Throughout the course of the biology programme he gained interest for plants and graduated with a thesis on the floating fern Azolla in 2008. This was followed by a MSc biology study, which centred on the cell biology of plants and their pathogens. During his first thesis with Dr. André van Lammeren at the Laboratory of Cell Biology he studied at the microscopical level how a new bacterial pathogen invaded the bark tissue of horse chestnut trees. This project evolved into developing and validating a heat-based method to eradicate the pathogen from trees. He performed a second thesis project at the Laboratory of Phytopathology in the group of Prof. Francine Govers. During this thesis he focussed on transcriptional responses of the potato late blight pathogen Phytophthora infestans to a bacterial metabolite and on the membrane topology of a putative Phytophthora transmembrane signalling protein. For his MSc internship he then moved to Exeter (UK) to join the laboratory of Prof. Gero Steinberg at the University of Exeter. There he studied the molecular motor dynein in the hyphae of the basidiomycete fungus Ustilago maydis. In the spring of 2011 he finalized his MSc studies from which he graduated cum laude. Later that year Jeroen started a PhD project in the Laboratory of Cell Biology at Wageningen University. Supervised by Prof. Marcel Janson and Dr. Tijs Ketelaar he started investigating how the microtubule cytoskeleton directs the site of cell wall assembly during plant cell division. To get acquainted with the moss Physcomitrella patens as model system for studying plant cell division, he started his $\mathrm{PhD}$ research in the laboratory of Gohta Goshima at the University of Nagoya in Japan in the winter of 2011-2012. The outcomes of his research are presented in this thesis. As of November 2017 Jeroen joined the laboratory of Christine Faulkner at the John Innes Centre in Norwich (UK) to investigate cell-cell communication in plants. 


\title{
Education Statement of the Graduate School \\ Experimental Plant Sciences
}

\author{
Issued to: Jeroen de Keijzer \\ Date: 17 November 2017 \\ Group: Cell Biology \\ University: Wageningen University \& Research
}

\section{1) Start-up phase}

- First presentation of your project

Title: Vesicle transport and the phragmoplast

- Writing or rewriting a project proposal

- Writing a review or book chapter

Title: Microtubule networks for plant cell division, Systems and synthetic biology, 2014

DOI: $10.1007 / s 11693-014-9142-x$

Dec 2013

05 Apr 2012

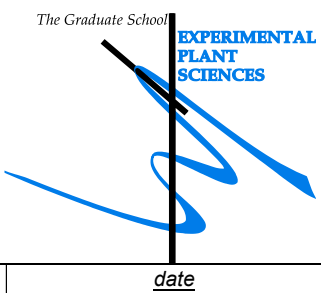

- National meetings (e.g. Lunteren days) and other national platforms

Annual meeting 'Experimental Plant Sciences', Lunteren, NL

Anual Dutch meeting on Molecular and Cellular Biophysics 2012, Veldhoven, NL

Annual meeting 'Experimental Plant Sciences', Lunteren, NL

Annual meeting 'Experimental Plant Sciences', Lunteren, NL

Anual Dutch meeting on Molecular and Cellular Biophysics 2014, Veldhoven, NL

Annual meeting 'Experimental Plant Sciences', Lunteren, NL

KNAW Biophysics Meeting, Amsterdam, NL

Annual meeting 'Experimental Plant Sciences', Lunteren, NL

Annual meeting 'Experimental Plant Sciences', Lunteren, NL

- Seminars (series), workshops and symposia

Seminar: 'DNA mechanics in vitro and kinesin action in vivo' - Erwin Peterman

Seminar: 'The architecture of plant actin networks' - Florian Hollandt

Symposium 'Frontiers in Plant Morphogenesis'

Seminar: 'Single-molecule biophysics: The fluorescence-based toolkit' - Johannes Hohlbein

Symposium 'Plant developmental biology', WUR, Wageningen

Seminar: 'Alpha aurora kinases direct division plane determination' - Daniel van Damme

ASCB education committee workshop 'Talking about evolution with doubsters'

Seminar: 'Mechanics of fission yeast spindle assembly' - Jon Ward

Seminar; 'Endosome trafficking drives fungal effector production during infection' - Gero Steinberg

Seminar: 'How plants read their own shapes' - Olivier Harmant

Seminar: 'Spatial control of cytokinesis in Arabidopsis' - Sabine Müller

Seminar: 'Long-term imaging of root development using light-sheet fluorescence microscopy' - Daniel von

Wangenheim

Seminar: 'Quality control systems that regulate microtubule dynamics and behaviour' - Niels Galjart

Seminar: 'Membrane trafficking in plant cells attacked by powdery mildew fungi' - Hans Thordal-

Christensen

\section{- Seminar plus}

Seminar 'Endosome trafficking drives fungal effector production during infection' - Gero Steinberg

- International symposia and congresses

Microtubules: Nucleation, Dynamics and Regulation; Nara inst. of science and technology, Japan

Fifth EMBO meeting, Amsterdam, NL

ASCB anual meeting 2014, Philadelphia, USA

RMS Botanical Microscopy Meeting 2015, Exeter, UK

- Presentations

Poster: 'High resolution imaging of cytokinesis using the moss Physcomitrella patens' - Lunteren, NL

Poster: 'Anti-parallel microtubules initiate cell wall formation for cell division in plants' - Veldhoven, NL

Poster: 'MAP65 defines initial site of cell wall deposition in the plant phragmoplast' - Amsterdam, NL

Talk: 'Regulation of microtubule dynamics at the plant phragmoplast midzone requires KIF4' Plant

developmental biology symposium, Wageningen, NL

Talk: 'Local regulation of microtubule dynamics in the phragmoplast of Physcomitrella patens ' PhD theme 1 symposium, Wageningen, NL

30 Nov 2012

29 Nov 2013

29-30 Jan 2015

17 Jan 2013

24 Jan 2014

08 Jan 2015

02 Apr 2012

01-02 Oct 2012

22-23 Apr 2013

15 Apr 2014

29-30 Sep 2014

13-14 Apr 2015

29 Mar 2016

11-12 Apr 2016

10 Apr 2017

13 Apr 2012

04 May 2012

13 Nov 2012

03 May 2013

14 Oct 2013

30 Jan 2014

07 Dec 2014

06 Feb 2015

05 Jun 2015

15 Mar 2016

09 Sep 2016

11 Oct 2016

28 Oct 2016

12 Dec 2016

05 Jun 2015

17 Feb 2012

21-24 Sep 2013

06-10 Dec 2014

19-23 Apr 2015

02 Apr 2012

01-02 Oct 2012

24 Sep 2013

14 Oct 2013

24 Jan 2014 
Poster: 'Control and function of microtubule overlaps in the cytokinetic phragmoplast network' -

Heidelberg, NL

Talk: 'Control and function of microtubule overlaps in the bipolar phragmoplast network' ASCB anual meeting, Philadelphia, PA, USA

Talk: 'Control and function of microtubule overlaps in the bipolar phragmoplast network', Annual meeting

'Experimental Plant Sciences', Lunteren, NL

Talk: 'Control and function of microtubule overlaps in the bipolar phragmoplast network' RMS Botanical

Microscopy Meeting 2015, Exeter, UK

Poster: 'Kinesin-4 mediated shortening of microtubule overlaps defines membrane accumulation sites during plant cytokinesis' - EMBO workshop, Vienna, Austria

Talk: 'Kinesin-4 mediated shortening of microtubule overlaps defines membrane accumulation sites

during plant cytokinesis' Department of Plant systems biology, VIB Ghent, Belgium

30 May 2014

09 Dec 2014

14 Apr 2015

21 Apr 2015

23 Jun 2016

29 Aug 2016

- IAB interview

Excursions

Subtotal Scientific Exposure

23.4 credits $^{*}$

\section{3) In-Depth Studies}

- EPS courses or other PhD courses

Workshop 'Plant Endomembrane Biology', VU Amsterdam, NL

Gordon research conference: Plant \& Microbial Cytoskeleton, Andover, USA

EMBO conference: Microtubules: Structure, regulation and functions, Heidelberg, Germany

EMBO Workshop 'New model systems for early land plant evolution', Vienna, Austria

- Journal club

Goshima-lab (Nagoya, Japan) literature discussion group

CLB (Wageningen, NL) literature discusssion group

- Individual research training

Training in moss culture and transformation techniques - lab of Gohta Goshima, Nagoya, Japan Short-term stay in Marine Biological Laboratory, Woods Hole, MA, USA

Subtotal In-Depth Studies

4) Personal development

- Skill training courses

ExPectationS (EPS Career day) 'Communication and Ethics in Science', Wageningen, NL

WGS PhD Workshop Carousel, Wageningen, NL

Adobe InDesign Essential Training, Wageningen, NL

- Organisation of PhD students day, course or conference

Design and implementation of a new practical for Cell biology and advanced imaging technologies

Membership of Board, Committee or PhD council

Herewith the Graduate School declares that the $\mathrm{PhD}$ candidate has complied with the educational requirements set by the Educational Committee of EPS which comprises of a minimum total of 30 ECTS credits

${ }^{*}$ A credit represents a normative study load of 28 hours of study. 
The research described in this thesis was financially supported by Human Frontier Science Program (HFSP) grant RGP0026/2011.

Financial support from Wageningen University for printing this thesis is gratefully acknowledged.

Cover: Jeroen de Keijzer

Chapter title pages: (Pelli D.G. (1999) Science 285(5429), pp. 844-846)

Printed by GVO drukkers \& vormgevers B.V., Ede, NL 